Sara Malvar Mauá

\title{
Hydrodynamics of living fluids in microflows
}

São Paulo

2019 


\section{SARA MALVAR MAUÁ}

\section{Hydrodynamics of living fluids in microflows}

Ph.D. Thesis presented to Escola Politécnica

- University of São Paulo in fulfillment of the requirements to obtain the degree of Doctor of Science.

Concentration area: Mechanical Engineering of Energy and Fluids.

Supervisors:

Professor Bruno Souza Carmo, PhD

Professor Francisco Ricardo Cunha, PhD

São Paulo

2019 
Autorizo a reprodução e divulgação total ou parcial deste trabalho, por qualquer meio convencional ou eletrônico, para fins de estudo e pesquisa, desde que citada a fonte.

Este exemplar foi revisado e corrigido em relação à versão original, sob responsabilidade única do autor e com a anuência de seu orientador.

São Paulo, de de

Assinatura do autor:

Assinatura do orientador:

\section{Catalogação-na-publicação}

\section{Mauá, Sara Malvar}

Hydrodynamics of Living fluids in microflows / S. M. Mauá -- versão corr.

-- São Paulo, 2019.

$205 \mathrm{p}$.

Tese (Doutorado) - Escola Politécnica da Universidade de São Paulo. Departamento de Engenharia Mecânica.

1.Fluidos ativos 2.Reologia (Modelos) 3.Nematoides 4.Método de fronteira imersa 5.Microfluidica I.Universidade de São Paulo. Escola Politécnica. Departamento de Engenharia Mecânica II.t. 


\section{Acknowledgments}

There are no words to convey my gratitude to all the people who, at some point, provided a word of support, an academic help or a few minutes of relaxation. I would like to express my sincere thanks to my supervisors, Professor Bruno and Professor Francisco, who have given me invaluable guidance, inspiration and suggestions in my quest for knowledge, but also freedom to pursue my research, while silently and non-obtrusively ensuring that I stay on course and do not deviate from the final objectives. I would also like to express my gratitude to Professor Vicente from Pathogens Molecular Analysis Laboratory (Biology Institute at University of Brasilia) who kindly assisted me in the cultivation of nematodes, providing the physical space and time of his student Anderson. I would like to express the deepest appreciation to Professor Paulo Arratia, from Penn Complex Fluids Lab (University of Pennsylvania) who not only welcomed me with open arms at Philadelphia, but also helped me and held fantastic discussions, as well as allowing me to use his laboratory and microfluidic rheometer.

I have great pleasure in acknowledging my gratitude to my colleagues and fellow research scholars Daiane, João and Gustavo from University of São Paulo and also Juliette, Ran and Bryan from University of Pennsylvania for the discussions, lunches and inspiring walks. I owe a very important debt to Rafael, who introduced me to my love, Fluid Mechanics, and has always been a mentor, colleague and great friend, guiding me, teaching me, listening to my cries and, more importantly, encouraging me and believing in my research. My heartfelt appreciation goes to my grandmother, my cousins, aunts and uncles who always understood my absences and provided solace in the difficult moments, strength in moments of weakness and laughter in moments of relaxation. I could not forget my friends in São Paulo and Brasilia who went through hard times together, cheered me on, and celebrated each accomplishment. I met each of you in a different educational moment of my life (elementary, high school, university, master and doctorate) and everyone who is with me to this day still inspires me in some way. Thanks to the friends of other departments of University of São Paulo for presenting me with different perspectives. In particular, thank you, Renata, for listening to my complaints and for making me laugh so much. Special thanks to Marina for the support and encouragement of the last 8 years. Cristina, who kept silent yet comforting company on those difficult days of writing and introduced me many new adventures on the top of mountains, which inspired me to understand nature, thank you.

My deepest appreciation goes to my mother and father. The longing I felt for you during those 
years became the strength to finish. Thank you for being beyond my parents, my great friends and companions, confidants and admirers. I hope this work makes you proud. I love you with all my heart. And I could not forget the little non-humans who provided warm and affectionate company throughout the whole journey: Fumaça, Canela, Sacola, Flicka and Branca, thank you for exhaling love.

I would like to thank Fundação de Apoio a Pesquisa do Estado de São Paulo - FAPESP for the financial support provided in this $\mathrm{PhD}$ (process number 2016/14337-5) and the University of Tokyo for the opportunity of research internship held in 2018 within the ambit of the SEELA program.

Finally, in obscure moments for science, I believe there is no purest protest than human creation. Unraveling the mysteries of nature is a form of art and protest. I thank all human beings who somehow understand the importance of those who have rebelled against what was already known in the search for new knowledge. My gratitude to all those who respect the work of scientists, researchers, professors and those who dared to see beyond, because It takes courage to want to discover and accept how things are. 
I dedicate this thesis to my 5-years-old version, that had already decided to be a scientist, and to my parents, who have encouraged my scientific spirit ever since. 


\section{Resumo}

MAUÁ, S. M. Hidrodinâmica de fluidos vivos em microescoamentos. 2019. 178 f. Tese de doutorado (Doutorado em Engenharia Mecânica de Energia e Fluidos ) - Escola Politécnica, Universidade de São Paulo, São Paulo, 2019.

A pincipal contribuição do presente trabalho é a proposição de um framework de análise de suspensões ativas utilizando como modelo vivo o nematoide Caenorhabditis elegans. Para tanto, cinco perspectivas diferentes são utilizadas: cinemática, macrorreológica, numérica, teórica e microrreológica. Primeiramente, uma análise teórica e experimental do movimento cinemático das partículas ativas suspensas em um fluido biológico é apresentada. Duas populações diferentes são examinadas: na ausência de alimento e com nematoides bem alimentados. Mostramos que a relação entre o comprimento de um nematoide individual e o comprimento de onda de seu movimento é linear e pode ser ajustada por uma previsão teórica proposta neste trabalho. Uma profunda discussão sobre a mecânica de propulsão com base em uma análise de escala que identifica três forças principais que atuam em um nematoide individual é feita. Além disso, investigamos a viscosidade de cisalhamento das suspensões de Caenorhabditis elegans. Os experimentos em cisalhamento oscilatório revelaram um comportamento anômalo da viscosidade com a variação da fração volumétrica de suspensão, $\phi$. A viscosidade efetiva da suspensão diminuiu com o aumento da fração volumétrica do nematoide para pequenas concentrações. Baseando-se nos dados experimentais, uma equação fenomenológica para a viscosidade efetiva da suspensão em função da fração volumétrica de partículas é proposta. $\mathrm{O}$ comportamento coletivo dos nematoides é também observado, em regime linear, pela diferença de tensões normais. Finalmente, o teste de step strain é conduzido para obter os tempos de relaxação. A presença de uma tensão ativa negativa devido ao comportamento impulsor do nematoide persiste por um certo período, levando a um undershoot negativo e a um comportamento oscilatório na função de relaxação. A fim de propor um modelo reológico, simplificações são efetuadas no modelo e simulações usando o método de fronteira imersa são conduzidas em um filamento flexível, variando o tipo de movimento que este realiza. Observa-se que a presença de assimetrias em seu movimento ondulatório gera drásticas mudanças em suas respostas cinemáticas. Um modelo reológico em função da orientação do filamento é proposto e validado com os dados experimentais em regime linear. Após a validação da equação constitutiva proposta, o modelo é observado sob o regime não-linear do cisalhamento oscilatório, no qual as caracterizações reológicas são feitas com 
base nos frameworks existentes, utilizando curvas de Lissajous-Bowditch e diagramas de Pipkin. Por fim, é apresentado um protocolo de análise de suspensões em um microrreômetro. Partículas são adicionadas e rastreadas à medida que um cisalhamento unidirecional (escoamento pulsátil) é aplicado. Os perfis de velocidade e taxa de cisalhamento são obtidos, assim como os sinais reológicos equivalentes à taxa de deformação e tensão. Ferramentas de análise de sinais são utilizadas e um sistema de inteligência artificial é proposto para remoção da componente constante do sinal adicionada pelo cisalhamento unidirecional, visando reconstruir o sinal com média temporal nula e possibilitando a aplicação de teorias reológicas já conhecidas, como a decomposição de tensões em coeficientes de Chebyshev para o cálculo das quantidades viscométricas de conformidade e fluidez. A principal contribuição do estudo diz respeito à observação, caracterização, modelagem e simulação de um animal microscópico que se movimenta de maneira diferente dependendo do ambiente e do fluido circundante. As propriedades reológicas analisadas, as simulações realizadas e o modelo proposto podem ser utilizados tanto para a produção de microorganismos artificiais quanto para o controle de organismos vivos. Além disso, essa combinação de análises e técnicas pode ser usada para estudo de qualquer tipo de suspensão ativa e passiva, fornecendo resultados novos e conclusivos em relação à caracterização reológica e ao comportamento físico das partículas.

Palavras-chave: Fluidos ativos. Reologia (Modelos). Nematoides. Método de fronteira imersa. Microfluidica. 


\section{Abstract}

MAUÁ, S. M. Hydrodynamics of living fluids in microflows. 2019. 178 p. Ph.D. thesis (Doctorade in Mechanical Engineering of Energy and Fluids) - Escola Politécnica, University of São Paulo, São Paulo, 2019.

The main contribution of the present work is the proposition of a framework for analysis of active suspensions using the Caenorhabditis elegans nematode as the living model. To do so, five different perspectives are used: kinematics, macro-reological, numerical, theoretical and micro-reological. First, a theoretical and experimental analysis of the kinematic motion of the nematodes suspended in a biological fluid is presented. Two different populations are examined: starving and well fed nematodes. We show that the relationship between the length of an individual nematode and the wavelength of its movement is linear and can be adjusted by a theoretical prediction proposed in this work. A deep discussion on propulsive mechanics based on a scale analysis that identifies three major forces acting on an individual nematode is made. In addition, we investigated the shear viscosity of Caenorhabditis elegans suspensions. The oscillatory shear experiments revealed an anomalous viscosity behavior with the variation of the volumetric fraction of suspension, $\phi$. The effective viscosity of the suspension decreased with increasing nematode volumetric fraction at low concentrations. Based on the experimental data, a phenomenological equation for the effective viscosity of the suspension as a function of the volumetric fraction of particles is proposed. The collective behavior of the nematodes is also observed in linear regime through the difference of normal stresses. Finally, step strain tests are conducted to obtain the relaxation times. The presence of a negative active stress due to the nematoid driving behavior persists for a period of time, leading to a negative undershoot and an oscillatory behavior in the relaxation function. In order to propose a rheological model, simplifications are made in the model and immersed boundary method simulations are conducted in a flexible filament, varying the type of movement that it performs. It is observed that the presence of asymmetries in its undulating movement generates drastic changes on its kinematic responses. A rheological model as a function of filament orientation is proposed and validated with experimental data in linear regime. After validation of the proposed constitutive equation, the model is observed under the nonlinear regime of oscillatory shear, in which the rheological characterizations are made based on existing frameworks using Lissajous-Bowditch curves and Pipkin diagrams. Finally, a protocol for analysis of suspensions in a microrheometer is 
presented. Particles are added and tracked as unidirectional oscillatory shear (pulsatile flow) is applied. The velocity and shear rate profiles are obtained, as well as the rheological signals equivalent to the strain rate and stress. Signal analysis tools are used and an artificial intelligence system is proposed to remove the component added to the signal by unidirectional shear, aiming to reconstruct the signal with null temporal average and allowing the application of well known rheological theories, such as the decomposition of stresses in coefficients of Chebyshev, for the calculation of viscommetric quantities of compliances and fluidities. The major contribution of the study concerns the observation, characterization, modeling and simulation of a microsized animal that moves in different fashion, depending on the environment, and the surrounding fluid. The rheological properties analyzed, simuations performed and model proposed can be used for both production of artifitial microorganisms and control of living organisms. Moreover, this combination of analyses and techniques can be used to study any type of passive and active suspension providing new and conclusive results regarding the rheological characterization and the physical behavior of the particles.

Keywords: Active fluids. Rheology (Models). Nematodes. Immersed boundary method. Microfluidic. 


\section{List of Figures}

1.1 Nematode's life cycle. . . . . . . . . . . . . . . . . . . 6

1.2 Nematode sinusoidal like motion. . . . . . . . . . . . . . . . 7

1.3 Nematode's head motion. . . . . . . . . . . . . . . . . 8

1.4 Main contributions of the thesis. . . . . . . . . . . . . . 15

2.1 Stress as a function of time for Giesekus model. . . . . . . . . . . . . . . 33

$2.2 \eta_{M}^{\prime}, \eta_{L}^{\prime}, G_{M}^{\prime}$ and $G_{L}^{\prime}$ in the Lissajous-Bowditch for Giesekus model with parameters. 36

2.3 Lissajous-Bowditch for UPC Maxwell model. . . . . . . . . . . . . . . . . . 37

2.4 Lissajous-Bowditch for Giesekus model with parameters. . . . . . . . . . . . . 39

2.5 Stress response of the UPC Maxwell model. . . . . . . . . . . . . . . . . . 41

2.6 Dimensionless Lissajous-Bowditch curve of the elastic stress component of the UPC Maxwell model. . . . . . . . . . . . . . . . . . . . . . 42

2.7 Dimensionless Lissajous-Bowditch curve of the viscous stress component of the UPC Maxwell model. . . . . . . . . . . . . . . . . . . . . . . . 43

2.8 Elastic and viscous Chebyshev coefficients of Giesekus model. . . . . . . . . . . 44

2.9 Stress as a function of time for Giesekus model. . . . . . . . . . . . . . . . . 46

2.10 Lissajous-Bowditch curves of Giesekus model. . . . . . . . . . . . . . . . . 47

3.1 Bending angle at the midpoint. . . . . . . . . . . . . . 51

3.2 Period of the sine wave the best fits the worm's posture. . . . . . . . . . 51

3.3 Cumulative distribution function (CDF) of the standard normal distribution and frequency histogram of well-fed sample. . . . . . . . . . . . . . . 52

3.4 Cumulative distribution function (CDF) of the standard normal distribution and frequency histogram of the starving sample. . . . . . . . . . . . . 53

3.5 Geometrical sketch of variables $a, \delta, \lambda, \theta$ and v . . . . . . . . . . . . 54

3.6 Average nematode size of both starving and well-fed nematodes as a function of $\lambda .60$

3.7 Nematode's bending angle $\theta$ and head to tail distance $R$ as a function of time. . . . 61

3.8 Trajectory of 10 starving nematode's centroid . . . . . . . . . . . . . . 62

3.9 Trajectory of 10 well-fed nematode's centroid. . . . . . . . . . . . . . 63

3.10 Comparison of head (a) and centroid (b) motion of one specific starving individual. $\quad 64$ 
3.11 Comparison of head (a) and centroid (b) motion of one specific well-fed individual. 65

4.1 Cultivation steps of both studied liquids. . . . . . . . . . . . . . . 70

4.2 Typical suspensions of $C$. elegans. . . . . . . . . . . . . . . 71

4.3 Shear elastic modulus, $G^{\prime}(\omega \rightarrow 0)$, and loss modulus, $G^{\prime \prime}(\omega \rightarrow 0)$, as a function of the volume fraction. The insert shows $G^{\prime}(\omega)$ of an aqueous polyacrylamide solution

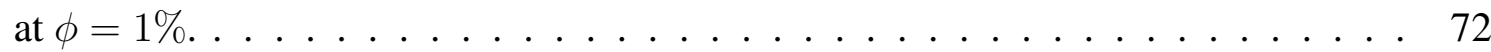

4.4 Dimensional first normal stress difference obtained through Cox-Merz rule $\phi=$ $3.5 \%$ as a function of the shear rate $\dot{\gamma}$. The insert shows the difference in $\Delta N_{1}=$ $N_{1}(\omega \rightarrow 2.5)-N_{1}(\omega \rightarrow 0) . \ldots \ldots \ldots \ldots$. . . . . . . . . . . . . . .

4.5 Non-dimensional first normal stress difference for $\phi=1 \%$. . . . . . . . . . 75

4.6 Effective viscosity as a function of $\phi \ldots \ldots \ldots$. . . . . . . . . . . 77

4.7 Relaxation function of $C$. elegans suspension of $\phi=1 \%$. . . . . . . . . . . 80

4.8 Slender body in a prestressed weighted state . . . . . . . . . . . . . 81

4.9 Active relaxation time as a function of $\phi \ldots \ldots \ldots$. . . . . . . . . . 82

4.10 Schematic of pusher and puller microorganisms. . . . . . . . . . . . . . 84

5.1 Ilustration of damped springs model. . . . . . . . . . . . . . . . . . 90

5.2 Ilustration of noninvariant beams model . . . . . . . . . . . . . . . 91

5.3 Nematode simplified model. . . . . . . . . . . . . . . . . 93

5.4 Nematode simplified model. . . . . . . . . . . . . . . . . . 94

5.5 Nematode curvature considering symmetric changes in the mediary points. . . . . 99

5.6 Forward distance swam in the case of symmetric interpolation points. . . . . . . 100

5.7 Forward velocity swam in the case of symmetric interpolation points. . . . . . . . 101

5.8 Zoom of the forward velocity in the case of symmetric interpolation points. . . . . 102

5.9 Nematode's trajectory in the symmetric case. . . . . . . . . . . . . 103

5.10 Nematode curvature considering asymmetric changes in the mediary points. . . . . 104

5.11 Forward distance swam in the case of asymmetric interpolation points. . . . . . . 105

5.12 Forward velocity swam in the case of asymmetric interpolation points. . . . . . . 106

5.13 Zoom of the forward velocity in the case of asymmetric interpolation points. . . . . 107

5.14 Nematode's trajectory in the asymmetric mediary points. . . . . . . . . . . 108

5.15 Nematode curvature considering asymmetric changes in the stroke period. . . . . . 109

5.16 Forward distance swam in the case of asymmetric stroke period. . . . . . . . . 110

5.17 Forward velocity swam in the case of asymmetric stroke period. . . . . . . . 111

5.18 Zoom of the forward velocity in the case of asymmetric stroke period. . . . . . 112 
5.19 Nematode's trajectory in the case of asymmetric stroke period. . . . . . . . . 113

5.20 Piecewise cubic interpolant, $g(t)$, velocity, $\dot{g}(t)$, and acceleration, $\ddot{g}(t)$, for symet-

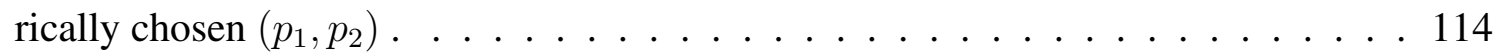

5.21 Piecewise cubic interpolant, $g(t)$, velocity, $\dot{g}(t)$, and acceleration, $\ddot{g}(t)$, for asymetrically chosen $\left(p_{1}, p_{2}\right) \ldots \ldots \ldots \ldots \ldots \ldots$

6.1 Entanglement of nematodes observed during experiments after shear. . . . . . . 120

6.2 Elastic Lissajous-Bowditch curves considering the presence of non-affine motion. . 122

6.3 Viscous Lissajous-Bowditch curves considering the presence of non-affine motion. 123

6.4 Elastic Lissajous-Bowditch in linear regime. . . . . . . . . . . . . . . . . 125

6.5 Pipkin diagram for the proposed model considering $D e_{a} \ldots \ldots$. . . . . . . . 126

6.6 Comparison between stress of experimental data and proposed model. . . . . . . 127

6.7 Comparison of the relative bulk viscosity between experimental data and proposed model. . . . . . . . . . . . . . . . . . . . . . . 129

6.8 Lissajous-Bowditch curves for nonlinear regime. . . . . . . . . . . . . . 131

6.9 Representation of the cage moduli, $G_{r}$, in Lissajous-Bowditch curves. . . . . . . 132

6.10 Plots of viscous Lissajous-Bowditch curves across the Pipkin space. . . . . . . . 133

6.11 Plots of elastic Lissajous-Bowditch curves across the Pipkin space. . . . . . . . . 134

6.12 Lissajous-Bowditch curve of the first normal stress difference and the shear strain. . 138

6.13 Plots of the Lissajous curves of first normal stress difference and shear stress. . . . 139

6.14 Shear stress, first normal stress difference, second normal stress difference and Fast

Fourier Transforms. . . . . . . . . . . . . . . . . . . . . 140

6.15 Shear stress and second normal stress difference. . . . . . . . . . . . . 141

7.1 Microchannel schematic ... . . . . . . . . . . . . . 143

7.2 Microchannel schematic . . . . . . . . . . . . . . . . . 144

7.3 Identified particles in water. The particles with especially low mass or especially large size are probably out of focus or aggregated, respectively. . . . . . . . . . . 148

7.4 Normalized and filtered input pressure for the Newtonian suspension. . . . . . . . 149

7.5 Normalized output velocity and input pressure for the Newtonian suspension. . . . 150

7.6 Normalized and filtered input pressure for the Newtonian suspension. . . . . . . . 151

7.7 Normalized output velocity and input pressure for the non-Newtonian suspension. . 152

7.8 Comparison between theoretical and experimental velocity pofiles for Newtonian fluid. . . . . . . . . . . . . . . . . . 153

7.9 Velocity and shear-rate profiles for Newtonian and non-Newtonian suspensions. . . 154 
7.10 Neural network scheme. . . . . . . . . . . . . . . . . . . . . . 158

7.11 Algorithm used for the data generation considering UPC Maxwell model. . . . . 160

7.12 Stress as a function of time obtained from the neural network for specific set of

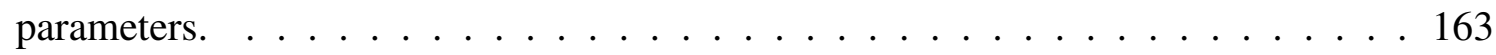

7.13 Stress as a function of time obtained from the neural network for specific set of parameters. . . . . . . . . . . . . . . . . . . . . . . 164

7.14 Filtered pressure, normalized velocity and expected velocity in simulated LAOS experiment for the Newtonian suspension. . . . . . . . . . . . . . . . . . 165

7.15 Filtered pressure, normalized velocity and expected velocity in simulated LAOS experiment for the non-Newtonian suspension. . . . . . . . . . . . . . . 166 


\section{List of Tables}

3.1 Nematodes motion characterization in the absence of food. . . . . . . . . . . 55

3.2 Well-fed nematodes motion characterization. . . . . . . . . . . . . 55

3.3 Characterization of the main dimensional quantities, forces and physical parameters averages of the nematodes locomotion. . . . . . . . . . . . . 57

6.1 Parameters used to fit the experimental data to the model . . . . . . . . . . 128

7.1 Parameters used to train the neural network . . . . . . . . . . . . . 159 


\section{Symbols}

\section{Kinematic Quantities}

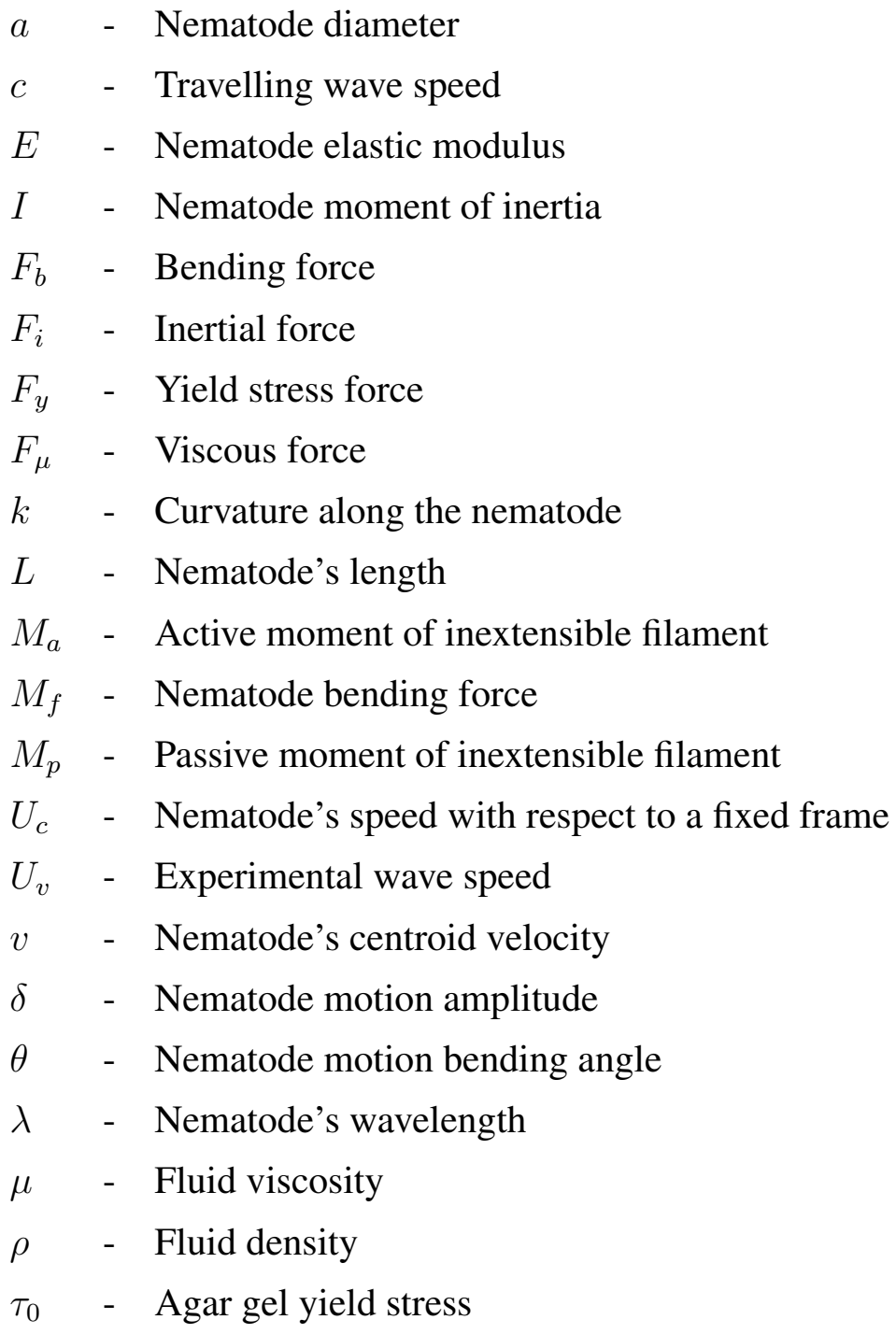


Acronyms

$\begin{array}{lll}\text { DWS } & - \text { Downstroke } \\ \text { FENE } & - \text { Finite extensibility nonlinear elastic } \\ \text { FT } & - \text { Fourier transform } \\ \text { IBM } & - \text { Immersed boundary method } \\ \text { IDL } & - & \text { Interactive data language } \\ \text { LAOS } & - & \text { Large Amplitude Oscillatory Shear } \\ \text { LCM } & - & \text { Lower-convective Maxwell } \\ \text { MPL } & - & \text { Multilayer perceptron } \\ \text { SAOS } & - & \text { Small Amplitude Oscillatory Shear } \\ \text { UCM } & - & \text { Upper-convective Maxwell } \\ \text { UD-LAOS } & - \text { Unidirectional Large Amplitude Oscillatory Shear } \\ \text { UPS } & - & \text { Upstroke }\end{array}$

\section{Dimensionless Groups}

$B e$ - Bending number

$B h$ - Bingham number

$C$ - Calibration parameters

De - Deborah number

$D e_{a}$ - Active Deborah number

Re - Reynolds number

$W i$ - Weissenberg number 


\section{Numerical Simulation Symbols}

\begin{tabular}{|c|c|}
\hline$a, b$ & - Two points of the nematode to be parameterized \\
\hline$C_{x}^{\delta}, C_{y}^{\delta}$ & - Curvatures \\
\hline$C_{x}, C_{y}$ & - Initial curvature configuration \\
\hline$C_{x}^{\prime}, C_{y}^{\prime}$ & - Final curvature configuration \\
\hline$d$ & - Extra length after streching of spring \\
\hline$d_{0}, d_{1}, d_{2}, d_{3}$ & - Interpolation cubic polynomial coefficients \\
\hline $\boldsymbol{D}^{0}$ & - Central differencing operator \\
\hline$D_{\alpha}^{ \pm}$ & - Forward and backward approximations to $\partial / \partial x_{a}$ \\
\hline$S_{\Delta x}$ & - Skew-symmetic difference operator \\
\hline $\boldsymbol{f}(s, t)$ & - Lagrangian forcing term \\
\hline $\boldsymbol{F}(x, t)$ & $\begin{array}{l}\text { - Force per unit area applied to the field by the immersed } \\
\text { boundary }\end{array}$ \\
\hline$h(t)$ & - Interpolation cubic polynomial \\
\hline$k_{\text {beam }}$ & - Beam stiffness coefficient \\
\hline$k_{s p r}$ & - Spring stiffness coefficient \\
\hline$k_{t}$ & - Stiffness coefficient \\
\hline$k_{X_{i}}, k_{Y_{j}}$ & - Fourier wave-numbers \\
\hline$k_{t}$ & - Stiffness coefficient \\
\hline$p(x, t)$ & - Pressure field \\
\hline$p_{1}, p_{2}$ & - Interpolation mediary points \\
\hline$R_{L}$ & - Resting length \\
\hline$s$ & - Lagrangian parameter \\
\hline$t$ & - Time \\
\hline$t_{u}, t_{d}$ & - Upstroke and downstroke period \\
\hline$T$ & - Total period time \\
\hline $\boldsymbol{U}(x, t)$ & - Fluid velocity field \\
\hline $\boldsymbol{U}^{n+1}$ & - Updated local velocity positions \\
\hline$x$ & - Position \\
\hline $\mathrm{r}(s, t)$ & - Cartesian coordinates at time $t$ and Lagrangian parameter $s$ \\
\hline
\end{tabular}




$\begin{array}{lll}\boldsymbol{X}_{B} & - & \text { Preferred curvature (bending) } \\ X_{e} & - & \text { Position of swimmer's head after } 4 \text { strokes } \\ \boldsymbol{X}_{M} & - & \text { Position in Cartesian coordinates of the master Lagrangian } \\ & & \text { nodes } \\ \boldsymbol{X}^{n+1} & - & \text { Updated material positions } \\ \boldsymbol{X}_{S} & - & \text { Position in Cartesian coordinates of the slave Lagrangian } \\ & & \text { nodes } \\ \delta(x) & - & \text { Three-dimensional delta function } \\ \varphi & - & \text { Streamfunction } \\ \boldsymbol{\omega} & - & \text { Vorticity }\end{array}$




\section{Macrorheological Quantities}

\begin{tabular}{|c|c|c|}
\hline$D$ & - & Strain rate tensor \\
\hline$G^{\prime}$ & - & Elastic shear modulus \\
\hline$G^{\prime \prime}$ & - & Loss modulus \\
\hline$I$ & - & Identity tensor \\
\hline$N_{1}$ & - & First normal stress difference \\
\hline$N_{2}$ & - & Second normal stress difference \\
\hline$n$ & - & Pressure \\
\hline$Q$ & - & Alignment order deviatoric tensor \\
\hline$q_{k}$ & - & Unit direction vector of $k$-th active particles \\
\hline$S$ & - & Stresslet \\
\hline $\bar{S}$ & - & Average stresslet \\
\hline$T$ & - & Suspension bulk stress tensor \\
\hline$u$ & - & Velocity field \\
\hline$\gamma$ & - & Strain amplitude \\
\hline$\gamma_{0}$ & - & Initial strain amplitude \\
\hline$\dot{\gamma}$ & - & Strain-rate amplitude \\
\hline$\eta$ & - & Viscosity fourth order tensor \\
\hline$\lambda$ & - & Reactive coefficient \\
\hline$\sigma$ & - & Total active stress \\
\hline$\sigma^{\prime}$ & - & Elastic component of the total active stress \\
\hline$\sigma^{\prime \prime}$ & - & Viscous component of the total active stress \\
\hline$\sigma_{x y}$ & - & Shear stress component \\
\hline$\sigma_{x x}, \sigma_{11}$ & - & Normal stress component \\
\hline$\sigma_{y y}, \sigma_{22}$ & - & Normal stress component \\
\hline$\sigma_{z z}, \sigma_{33}$ & - & Normal stress component \\
\hline$\tau_{a}$ & - & Characteristic relaxation time of the active particle \\
\hline$\tau_{c}$ & - & Characteristic relaxation time of the material \\
\hline$\tau_{d}$ & - & Characteristic time of deformation \\
\hline$\tau_{0}$ & - & Characteristic time of observation \\
\hline$\phi$ & - & Volume fraction \\
\hline
\end{tabular}




\begin{tabular}{|c|c|}
\hline$\xi$ & - Slip parameter in Gordon-Schowalter derivative \\
\hline$\omega$ & - Oscillatory frequency \\
\hline$e_{m}, v_{m}$ & - Elastic and viscous Chebyshev coefficients \\
\hline$G_{L}^{\prime}$ & - Large-rate shear elastic modulus \\
\hline$G_{M}^{\prime}$ & - Minimum-rate shear elastic modulus \\
\hline$G_{r}$ & - Cage moduli \\
\hline$I_{m}^{\prime}, I_{m}^{\prime \prime}$ & - Real and imaginary harmonics \\
\hline$S(\omega, \gamma)$ & - Strain-stiffening ratio \\
\hline$T(\omega, \gamma)$ & - Shear-tickening ratio \\
\hline$\beta$ & - Experimental constant related to the stresslet strengh \\
\hline$\gamma_{y}$ & - Yielding point \\
\hline$n$ & - Newtonian fluid viscosity \\
\hline$\eta_{L}^{\prime}$ & - Large-rate dynamic viscosity \\
\hline$\eta_{M}^{\prime}$ & - Minimum-rate dynamic viscosity \\
\hline$\eta_{r}$ & - Suspension effective viscosity \\
\hline nax & - Maximum stress \\
\hline
\end{tabular}




\section{Microrheological Quantities}

\begin{tabular}{|c|c|c|}
\hline$a_{n}$ & - & Modified Chebyshev coefficients \\
\hline$A w$ & - & Box used to model the background of the captured image \\
\hline$B$ & - & Normalization coefficient \\
\hline$c_{n}$ & - & Chebyshev coefficients \\
\hline$d$ & - & Depth of the channel \\
\hline$L$ & - & Length of the channel \\
\hline$m_{0}$ & - & Integrated brightness \\
\hline$N$ & - & Number of particles \\
\hline$p$ & - & Measured pressure \\
\hline $\boldsymbol{r}(t)$ & - & Location of te $i$-th particle \\
\hline$S_{\text {old }}$ & - & cumulative sum of current and past squared gradients \\
\hline$S_{n}$ & - & Chebyshev serie \\
\hline$T_{n}$ & - & Chebyshev polynomials \\
\hline$U$ & - & Characteristic velocity of the flow \\
\hline$U_{x}$ & - & Velocity profile \\
\hline$v$ & - & Measured velocity \\
\hline$w$ & - & Size used to filter the background noise \\
\hline$w_{d}$ & - & Width of the channel \\
\hline$w_{\text {new }}, w_{\text {old }}$ & - & New and old network weights \\
\hline$x_{i}$ & - & Input neuron \\
\hline$\alpha_{L R}$ & - & Network learning rate \\
\hline$\epsilon$ & - & "Fuzz factor" \\
\hline$\lambda_{n}$ & - & Correlation length \\
\hline$\rho(\boldsymbol{r}, t)$ & - & Particle trajectory \\
\hline
\end{tabular}




\section{SUMMARY}

List of Figures

$\begin{array}{ll}\text { List of Tables } & \text { Xv }\end{array}$

List of Symbols $\quad$ xxi

$\begin{array}{ll}\text { SUMMARY } & \text { xxviii }\end{array}$

1 Introduction 1

1.1 Active Fluids 1

$\begin{array}{ll}1.2 \text { Caenorhabditis elegans } & 4\end{array}$

$\begin{array}{llr}1.3 & \text { Applications } & 9\end{array}$

1.4 Rheology of active suspensions 11

$\begin{array}{lll}1.5 & \text { Microrheology } & 13\end{array}$

$\begin{array}{ll}\text { 1.6 General objetives } & 14\end{array}$

2 Theoretical Foundations $\quad 17$

$\begin{array}{lll}\text { 2.1 Constitutive Formalism } & 17\end{array}$

2.1.1 Casuality principle . . . . . . . . . . . . . . . 17

2.1.2 Local action principle . . . . . . . . . . . . . . . . . 18

2.1.3 Material Frame Indifference principle . . . . . . . . . . . . . . . . 20

2.1 .4 Fading Memory . . . . . . . . . . . . . . . . . . . . . 21

$\begin{array}{ll}\text { 2.2 Constitutive Models } & 22\end{array}$

$\begin{array}{llr}2.3 & \text { Rheological Flows } & 27\end{array}$ 
2.3.1 Small Amplitude Oscillatory Shear (SAOS) . . . . . . . . . . . . . . . 28

2.3.2 Step Strain Tests . . . . . . . . . . . . . . . . . . . . . 30

2.3.3 Large Amplitude Oscillatory Shear (LAOS) . . . . . . . . . . . . . . . . . 32

2.3.3.1 Fourier Transform Framework . . . . . . . . . . . . . . . . . 33

2.3.3.2 Lissajous-Bowditch Analysis . . . . . . . . . . . . . . . . 35

2.3.3.3 LAOS Strain Chebyshev Framework . . . . . . . . . . . . . . . . . 38

2.3.3.4 LAOS Stress Chebyshev Framework . . . . . . . . . . . . . . . . . 43

2.3.4 Unidirectional Large Amplitude Oscillatory Shear (UD-LAOS) . . . . . . . . . . 44

3 Kinematic Analysis $\quad 49$

3.1 Experimental methods $\quad 50$

3.1 .1 Preparation of growth media . . . . . . . . . . . . . . . 50

3.1 .2 Experimental protocol ......................... 51

3.2 Results and discussions $\quad 54$

3.2.1 Preliminary characterization . . . . . . . . . . . . . . 54

3.2 .2 Scalings arguments . . . . . . . . . . . . . . . . 55

3.2.3 Theoretical prediction - Length and wavelength relation . . . . . . . . . 58

3.2.4 Non-harmonic motion analysis . . . . . . . . . . . . . . . . 61

$\begin{array}{lll}\text { 3.3 Chapter conclusions } & 66\end{array}$

4 Rheological Analysis $\quad 69$

$\begin{array}{llr}4.1 & \text { Experimental Procedure } & 69\end{array}$

4.2 Results and discussions $\quad 71$

4.2.1 Comparison between active and passive suspension . . . . . . . . . . . . 71

4.2.2 Effective viscosity for different volume fractions . . . . . . . . . . . . 75

4.2.3 Stress relaxation function and particle activity relaxation . . . . . . . . . 79

4.2.4 Particle dipole stresslet - a physical interpretation . . . . . . . . . . . . . 82

$\begin{array}{llr}\text { 4.3 Chapter conclusions } & 85\end{array}$

5 Filament Kinematic Simulation $\quad 87$ 
$\begin{array}{llr}5.2 & \text { Swimmer modelling } & 92\end{array}$

$\begin{array}{lll}5.3 & \text { Results and discussions } & 99\end{array}$

5.3.1 Symmetrical changes . . . . . . . . . . . . . . . . . . . 99

5.3 .2 Asymmetrical changes . . . . . . . . . . . . . . . . . . 104

5.3.3 Asymmetric Stroke Period . . . . . . . . . . . . . . . . . . 109

$\begin{array}{lll}5.4 \text { Chapter conclusions } & 114\end{array}$

$\begin{array}{llr}6 & \text { Nonlinear Constitutive Model } & 117\end{array}$

6.1 Non-affine Motion $\quad 119$

$\begin{array}{llr}\text { 6.2 } & \text { Linear Regime } & 124\end{array}$

6.3 Nonlinear Regime 130

$\begin{array}{ll}\text { 6.4 Normal Stress Differences } & 135\end{array}$

$\begin{array}{ll}\text { 6.5 Chapter conclusions } & 137\end{array}$

7 Microrheometer $\quad 143$

$\begin{array}{lll}\text { 7.1 Particle tracking analysis } & 145\end{array}$

$\begin{array}{lll}7.2 & \text { Newtonian and polymeric suspensions } & 147\end{array}$

$\begin{array}{lll}\text { 7.3 Chebyshev expansion for UD-LAOS } & 155\end{array}$

$\begin{array}{lll}7.4 & \text { Neural networks } & 157\end{array}$

7.4.1 Methodology validation . . . . . . . . . . . . . . . 161

7.4 .2 Experimental data $\ldots \ldots \ldots \ldots$. . . . . . . . . . . . . . . . . . . . . . . . . . .

$\begin{array}{llr}\text { 7.5 Chapter conclusions } & 165\end{array}$

8 Conclusion and future work $\quad 167$ 
References

A Spectral Methods via Fast Fourier Transform (FFT)

B Discretizing Navier-Stokes equations $\quad 201$

$\begin{array}{lll}\text { C Spline Interpolant Coefficients for Swimmers } & 203\end{array}$

C.1 Symmetric $\left(p_{1}, p_{2}\right)$ coefficients

C.2 Asymmetric $\left(p_{1}, p_{2}\right)$ coefficients 


\section{Introduction}

\subsection{Active Fluids}

Active fluids have attracted much attention in recent years for their interesting and often unexpected dynamics (S AINTILLAN, 2010). An active particle can be considered as a particle which consumes energy from the surrouding environment to perform work or uses its own stored energy to promote its motion. Basically, the term active matter may describe natural or artificial systems that are out of thermodynamic equilibrium. Living entities such as birds, fish or bacteria intrinsically exist out of equilibrium by converting chemical content of their food into some form of mechanical work (Doostmohammadi et al., 2018). Similarly, synthetic systems can be designed to perform work driven by energy from light or chemical gradients (LADOUX AND MEGE, 2017).

A classic example of an active matter system is a flock of birds. A flock is composed of thousands of individual birds which act autonomously. Each bird is consuming its own on-board energy supply in order to move. Despite the fact that each bird is able to fly independent of the other birds, the collection of birds maintains a coherent, amorphous shape. In this thesis, we will focus on viscous fluids and swimmers. We define a "swimmer" to be a creature or object that moves by deforming its body in a periodic way (LAUGA AND POWER, 2009).

Many microscopic swimmers use one or more appendages for propulsion (FIELDING et $a l ., 2011)$. The appendage could be a relatively stiff helix that is rotated by a motor embedded in the cell wall, as in the case of Escherichia coli, or it could be a flexible filament undergoing whip-like motions due to the action of molecular motors distributed along the length of the filament, as in the spermatozoa of many species. In addition, the swimmer may move its whole body to produce bending waves and promote propulsion.

An important feature of materials built from active entities is the emergence of collective motion, in which groups of active particles move together as a unit on scales that are significantly larger than the size of an individual. Everyday examples are the intricate patterns formed by airborne starling flocks or when fish move together to avoid a predator. Similar collective behaviour persists down to micro-scales, where bacterial suspensions, tissues and intra cellular filaments use their intrinsic activity to create motions with lengths larger than individual cells or proteins. 
Complex fluids, such as intestinal fluid, human mucus and even mud (FU et al.; FAUCI AND Dillon; JUAREZ et al.; MALADEN et al.; LAUGA, 2009; 2006; 2010; 2009; 2007) were the basis of evolution of several microorganisms. The rheology of such fluids can substantially affect the swimming behaviour of a microorganism (KEIM et al., 2012). Considering a viscoelastic medium, the regular beating pattern exhibited by a freely swimming spermatozoon is replaced by a highamplitude and asymmetric bending of its flagellum (SZNITMAN et al., 2010). An interesting aspect on the mechanics of swimming is that the biomechanics of a living being must be adapted to its surroundings. For instance, small bodies seeking to swim in a viscous fluid are subjected to a principle known as kinematic reversibility in low Reynolds numbers (SHAPERE AND WILCZEK, 1987). This principle is related to the linearity of the Stokes equation and forces microoganisms to produce highly nonlinear motions in order to break the time-reversibility to which they are bonded. Some rich examples of this nonlinear motion include flagellar (KELLER AND SEGEL; BLAKE AND SlEIGH, 1971; 1974) and ciliary (BlAKE; DAUPTAIN et al., 1975; 2008) propulsion.

Many of the biological fluids are far from homogeneous. Highly heterogeneous biological materials include mucus, which forms a three-dimensional network with a potentially fractal length-scale distribution, and the cytoskeleton, which is an active structure that undergoes continuous remodeling in response to external and internal stimulation. Nevertheless, with the introduction of a more involved microstructure, such as the inclusion of long chain molecules (e.g., DNA, proteins, microtubules, nematodes, etc.), continuum assumptions are commonly made to make mathematical modeling and analysis of these complex fluids possible.

As said before, most studies of active fluids have focused on suspensions of motile particles (ABKENAR AND MARX, 2013) whose active stresses, produced by swimming, and spontaneous flows can enhance mixing (SOKOLOV AND ARANSON; SAINTILlan, 2009a; 2010), and affect chemotactic aggregation (LUSHI et al., 2012). A paradigmatic example is a suspension of selfpropelled microorganisms (bacteria, microphytes and nematodes). Experiments on these systems have cast light on a number of peculiar phenomena including: large-scale flows and collective motions on length scales much greater than the particle dimensions, very large density number fluctuations, enhanced swimming speeds, enhanced passive tracer diffusion and efficient fluid mixing. These effects may have an important impact on the growth of microorganismal colonies via nutrient and oxygen transport and mixing, both of which are enhanced by these chaotic flows.

Considering the forces exerted by the particle on the surrounding fluid and their directions, a single particle can be either extensile - if the forces are exerted from the centre of mass to the fluid 
- or contractile - if they are exerted from the fluid to the centre of mass of the particle (FIELDING $e t$ $a l ., 2011)$. The rheology of active fluids is also expected to display a very intriguing phenomenology (MARENDUZzo et al.; GiOMi et al.; CATES et al., 2007; 2008; 2008). Extensile and contractile particles lead to very different rheological responses. For instance, it has been predicted theoretically (VOITURIEZ et al., 2006) that active fluids should undergo a nonequilibrium phase transition between a "passive" quiescent phase, where the motion of each of the particles is basically uncorrelated and the coarse grained mean velocity field is uniform and zero, as in conventional passive unforced fluids, and an active phase, in which long-range correlations lead to a non-zero spontaneous flow in steady state.

Active particles with elongated shape can exhibit orientational order at high concentrations and have been linked to "living liquid crystals" (GRULLER et al., 2012). Their rich collective behavior includes nonequilibrium phase transition and pattern formation on mesoscopic scales. The activity of the system has been modeled using continuum equations proposed to describe specific microscopic models (LIVERPOOL AND MARCHETTI; LIVERPOOL AND MARCHETTI, 2003; 2007) or the hydrodynamic of liquid crystals. In this condition, there are still open questions regarding a constitutive model for these suspensions. However, a thorough study of the phenomenological characteristics of these microorganisms can produce advances in the proposed theories.

For suspensions of swimming microorganisms hydrodynamic theories were developed either based on the macroscopic transport equations phenomenology (SIHMA AND RAMASWAMY; YATES et al.; TONAR et al., 2002; 2009; 2005) or directly coming from a kinetic theory accounting explicitly for the transfer of momentum to the fluid and the hydrodynamic interactions between the swimmers (SAINTILLAN AND SHELlEY; SUBRAMANIAN AND Koch; GACHELIN AND ET AL., 2008; 2009; 2014). Numerical simulations of swimmers in a surrounding fluid were also performed to model active suspensions with the aim to test the theoretical predictions or to expand the studies to limits where the analytical theory is difficult (HERNANDEZ-ORTIZ et al., 2005). In particular, the issue of collective organization of swimmers was addressed beyond the linear stability analysis of hydrodynamic modes (SAINTILLAN AND SHELLEY, 2011). An important outcome of these studies is that, for rear-activated swimmers or "pushers", long-range nematic ordering as well as fully isotropic states are both unstable and essentially lead to collective motion. The onset of collective motion may or may not depend on the system size (HOHENEGGER AND SHELLEY; BASKARAN AND MARCHETTI, 2010; 2009).

In particular, it is interesting to investigate which types of structures microorganisms can 
form, which kinds of dynamics they can display and, given a particular set of microscopic elementary units, what range of possible macroscopic architectures, patterns and functionalities can be generated. While equilibrium statistical mechanics predicts the behaviour of "dead" materials, there is no analogous theory for non-equilibrium self-organized hierarchical systems of active matter. In addition, the principal organizing role in the formation of large-scale patterns (e.g., (CATES et al.; DOMBrowsKi et al., 2008; 2004)) is believed to be played by hydrodynamic interactions between individual swimmers and the environment. This includes the boundary effects as well as the hydrodynamic interaction with other swimmers (KELLER AND SEGEL; GYRYA et al., 1971; 2009).

A suspension of microorganisms have nonconventional properties, such as anomalous viscosities, a material property which describes the resistance of a fluid to shearing flows (TONAR et $a l$; SIHMA AND RAMASWAMY, 2005; 2002). The microorganisms have the ability to self-organize into ordered states (LIVERPOOL AND MARCHETTI, 2003), with local alignment, forming patterns (LIVERPOOL AND MARCHETTI, 2007) and favoring collective transport on scales larger than individual (MARENDUZzo et al., 2007). There is a wide and growing body of theoretical work focused on investigating the collective dynamics, picturing the individuals as static force multipoles (SOKOlov et al.; SAINTILlAn AND ShElley, 2009; 2008) interacting in a fluid or by generic rules of alignment. However, at the microscopic level, the dynamics of active individuals is often time dependent.

Despite this considerable body of work that has been carried out to investigate active fluids, this is a relatively new field of research there are still many points that need to be better understood. This projects intends to address a few of them proposing a framework for the complete study of active suspensions. For that, techniques of macroreology, microreology, particle tracking, signal analysis and machine learning will be used.

\subsection{Caenorhabditis elegans}

Several works have focused on studying $C$. elegans regarding its physical properties and aspects of low Reynolds number locomotion. Nematodes inhabit virtually every environment and are among the ubiquitous organisms on earth. The reasons for the use of this nematode as a benchmark goes from the easiness to manipulate them to the simplicity of its motion. We may say that mere 
$100 \mathrm{~g}$ of soil will typically house 300 individuals. Higher deformation speeds associated with lower internal viscous resistance are related to the shear thinning feature of an individual worm. The shape of an undulating crawler is defined by a dynamic balance between elastic, hydrodynamic, and muscular forces. As a result, the shear thinning property of the worm may influence the dynamics of motility, and shear thinning should be integrated into a full locomotory model. In a recent work (BACKHOLM et al., 2013) researchers investigated the response of a single C. elegans worm to the application of tangential stresses and found out that the elastic feature of the nematodes body may be responsible for a shear thinning behavior of a suspension of $C$. elegans. Moreover, as pressure and shear strength between worm and agar increases, so does the friction, leading to the presence of greater yield-stresses. When nematodes are on the surface of this medium they simply crawl. However, when they move inside the medium the forces exerted by their muscles end up breaking the bondings of the agar molecules and water is released in a process called syneresis.

Similar to other nematodes, the life cycle of $C$. elegans is comprised of the embryonic stage, four larval stages (L1-L4) and adulthood, as shown in figure 1.1, but individual sexes are not easily distinguished until the L4 stage. The lifecycle takes about 3 days at 20 degrees Celsius. The nematode also has an alternative L3 stage, known as the dauer (enduring) stage. This stage is anadaptation to survival of extreme conditions (mainly lack of food) and is likely in the wild to be C. elegans' dispersal stage. Dauer larval development is induced by crowding (more congeneric nematodes make dauer entry more likely), lack of food (less food as a L1 makes dauer development more likely), temperature (higher temperatures make dauer development more likely) and genetics (CASSADA AND RUSSEL, 1975).

One of the interesting features present in the active particles problem is the decision-making mechanism. A young C. elegans adult will perform an intensive search of where it believes food is likely to be found. This strategy, called "local search", is characterized by the worm making numerous sharp turns that keep it in its target search area. If the worm has not found food after 15 minutes, it abruptly switches its behavior to a so-called "global search" strategy, which features fewer sharp turns and more forays into the surrounding area (CALHOUN et al., 2014).

Berri et al. (BERRI et al., 2009) argue that C. elegans forward locomotion in low Reynolds number flow is achieved through modulation of a single gait. They claim that its interaction with the surrounding media is highly complex, despite its biological simplicity (it has only 302 neurons), which allows it to be a very efficient swimmer even subjected to a kinematic reversibility due to its small size. As a collateral effect, the study of $C$. elegans has also produced important advances on 
Figure 1.1: Nematode's life cycle. [Source: (STIERNAGLE, 2006).]

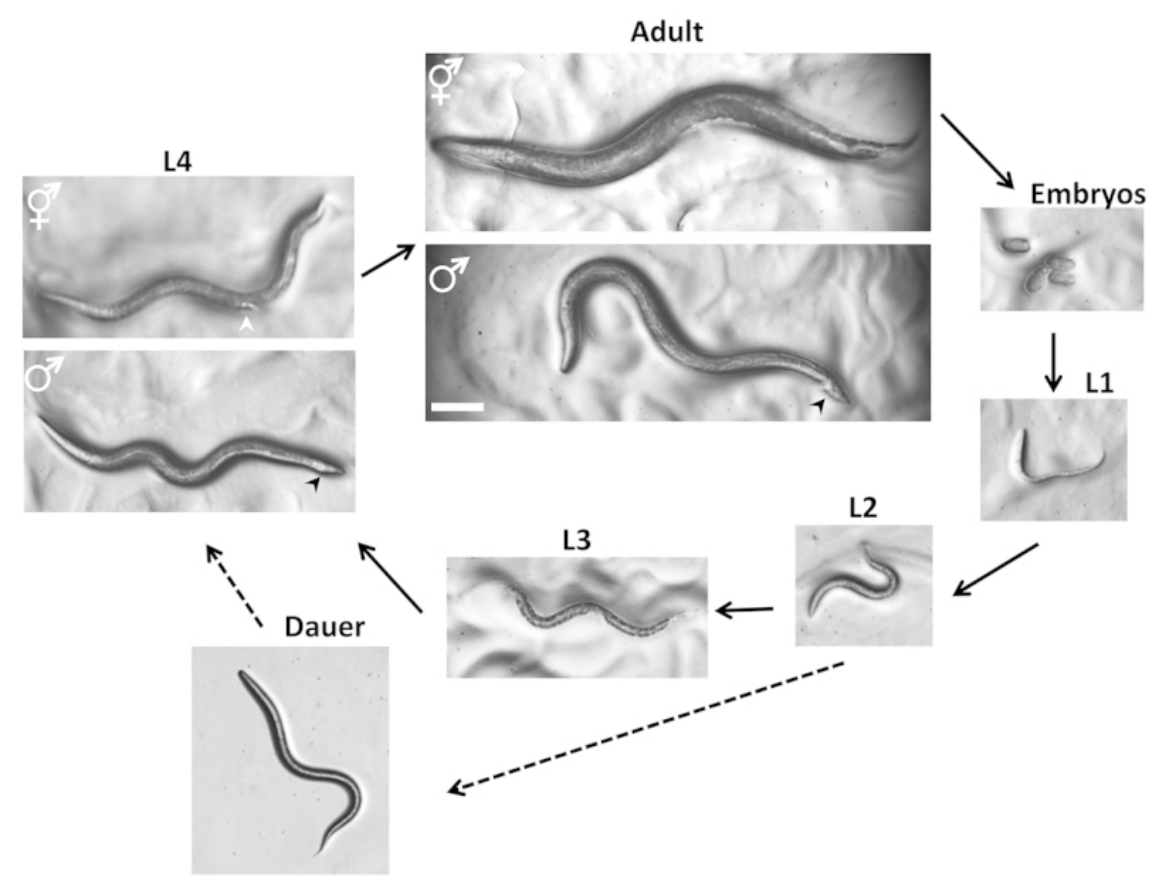

image analysis techniques of moving bodies for biological purposes (BISWAS et al., 1998). Some of these techniques have shown us the efficiency of its ondulatory motion, as seen in figure 1.2, which has inspired the production of moving micro and nano robots that use the same propelling principles (BOYLE et al., 2013) .

Considering that the Reynolds number related to the nematode's induced flow $R e \ll 1$, we shall refer this study as in the Stokesian realm, in contrast to the theories of inviscid flow, which might be termed as Eulerian realm. Most microorganisms move due to a periodic or near periodic motion of organelles such as cilia and flagella. Indeed time-reversal symmetry plays a key role in the selection of swimming strategies. Swimming cells, such as bacteria (prokaryotes) or spermatozoa (eukaryotes), represent the prototypical example of active soft matter (PURCELL, 1977). In addition, in the absence of inertia, the swimmer remains perpetually force and torque-free (LAUGA AND POWERS, 2009). In this condition, swimmers should change their shapes in a non-reciprocal fashion. In the case of $C$. elegans, flexibility or elasticity can lead to non-reciprocal shape change and thus to locomotion, as shown in figure 1.3.

Microorganisms, such as nematodes, respond to stimuli by swimming in particular directions. 
Figure 1.2: Nematode sinusoidal like motion. The figures $(485 \mu \mathrm{m} \times 645 \mu \mathrm{m})$ indicate six different time steps during the recording of the crawling motion of a nematode with total length of $400 \mu \mathrm{m}$. The time-step $\Delta t$ between the images is $2.5 \times 10^{-3} \mathrm{~s}$ and the sequence ilustrates the crawling motion of single worm in the agar gel medium. [Source: the author.]

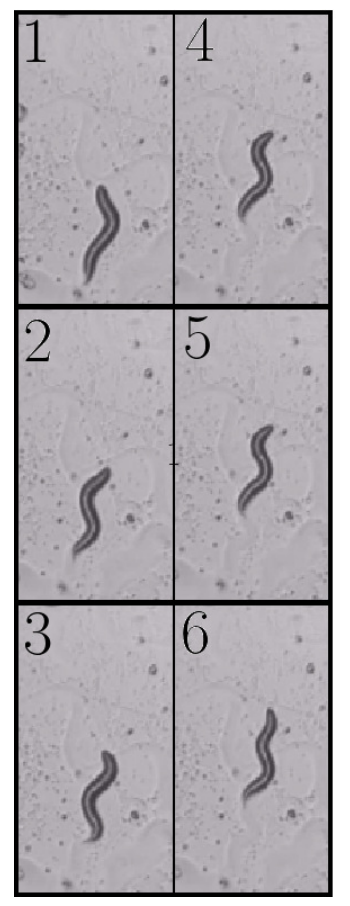

Such responses are called taxes. This ability to detect and respond to changes in the environment is a basic necessity for survival of all organisms, and as a result, a variety of mechanisms have evolved by which organisms sense their environment and respond to signals they detect. Often the response involves movement toward a more favorable environment or away from a noxious substance (ERBAn AND OThMer; PirRi AND Alkema; LeE et al., 2006; 2012; 2011). Taxes of importance are gravitaxis (or geotaxis), a response to gravity or acceleration; phototaxis, a response to light; and chemotaxis, a response to chemical gradients (PARIDA AND PADMANABHAN; PARIDA et al., 2016; 2017). Responses to shear in the ambient flow are sometimes called rheotaxis. Compensating torques due to shear and gravity produce gyrotaxis. According to Vidal-Gadea et al. (VIDAL-GADEA et al., 2015), some bacteria contain magnetic particles (i.e. magnetosomes), which cause them to swim along magnetic field lines (magnetotaxis).

In this work, we will refer to $C$. elegans as a nematic particle. Nematic particles are rod-like with head-tail symmetry. For some ranges of temperature or concentration they can predominantly align in a given direction, termed the director, resulting in a nematic phase with long-range orien- 
Figure 1.3: Nematode's head motion from top to bottom and left to right, respectively. It is interesting to observe that even when the body maintains its sinusoidal motion in one frame, the head produces several movements in diferent directions when searching for food. This different motion induces secondary frequencies. The time-step $\Delta t$ between figures $(485 \mu \mathrm{m} \times 645 \mu \mathrm{m})$ is $2.5 \times 10^{-3}$ second. [Source: the author.]

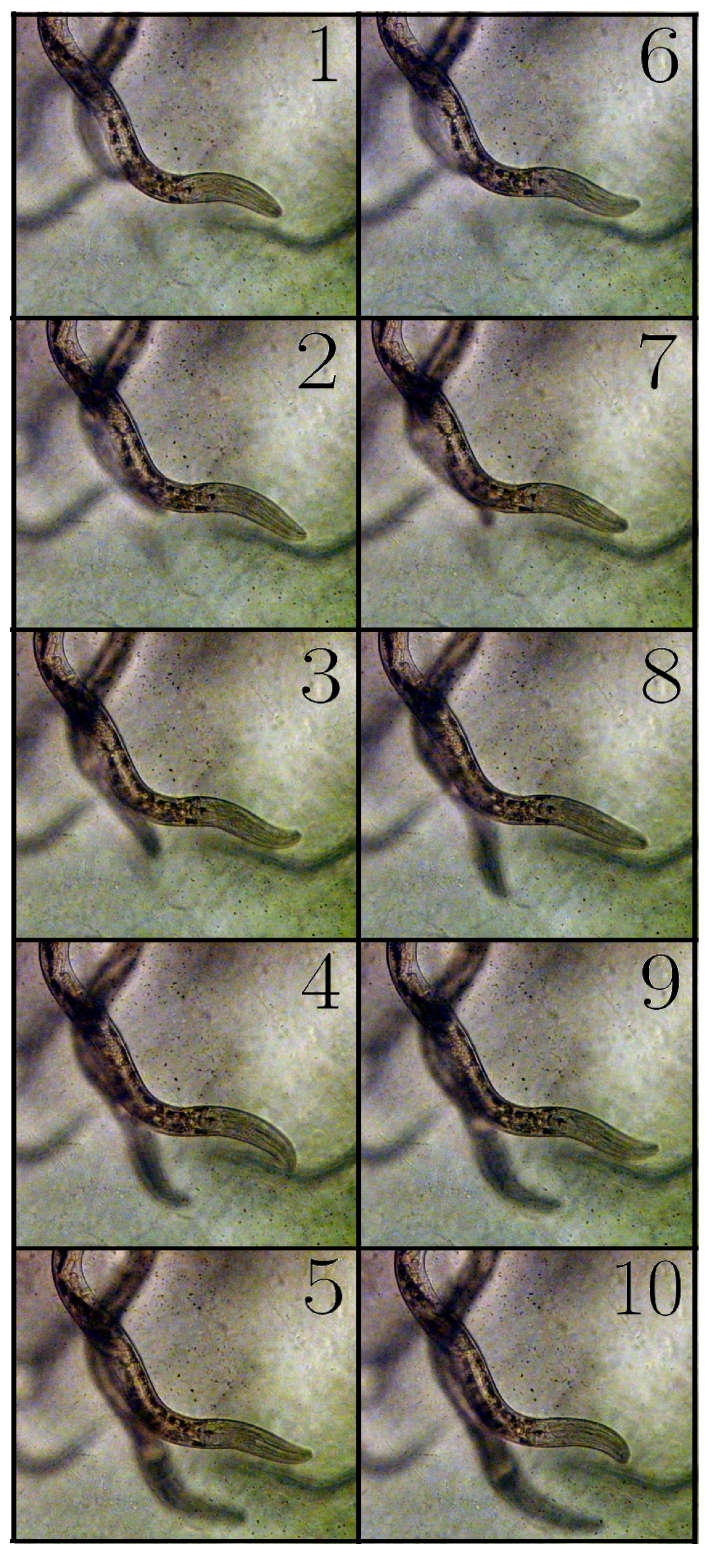

tation, but no long-range positional order. 


\subsection{Applications}

From a technological and engineering standpoint, active suspensions play an integral role in medical, industrial, and geophysical settings. The essential role that self-organization has in cell biology suggests that the use of internally driven components is the key to building materials with functionalities that have so far been confined to living organisms. The remarkable and diverse properties of cells arise from a highly conserved and fairly limited set of microscopic components. Evolutionary processes constrain the number of self-organized structures found in living organisms as compared with the much vaster manifold of all possible dynamical states that can be assembled from the same set of building blocks. Systematically assembling biological materials from the bottom up allows exploration of the phase space of a great number of possible dynamical states. Thus, in vitro studies not only have the potential for reproducing the properties and behaviours of biological materials found in living organisms, but also for uncovering entirely new phenomena that lack direct biological relevance. Such work does not just advance materials science; the simplified, well-controlled nature of in vitro systems allows detailed tests of principles of active matter, which, in turn, provides a rigorous foundation for understanding complex self-organizing processes taking place inside cells.

For instance, synthetic active matter systems have been constructed from the components of the cytoskeleton, that is, filamentous polymers and their associated proteins. Similar to the cellular cytoskeleton, such synthetic materials are driven away from equilibrium by energy-consuming processes. In order to build these synthetic systems, we must understand how they move and change the rheology of the surrouding fluid. As a matter of fact, with huge potential energy savings for technologies ranging from sensors to supercomputers, these self-propelling fluids are a new type of energy-harvesting machines. Wu (WU et al., 2017) created small machines composed of filaments and molecular motors. For the first time, fluid with directionless flow autonomously flowed unidirectionally through meter-long $3 \mathrm{D}$ channels until the chemical energy was depleted. The direction of the flow could be controlled with notches in the pipe. Its strength and speed could be regulated by the structure of the machines, referred to as "soft" because their operation was driven by structural alterations arising from changes in temperature or forces such as mechanical stress.

The study of the interaction between microorganisms and surrounding fluids is also applied in medical solutions. For instance, the dynamics of spermatozoon is directly associated with fertility problems. Coitus induces fluid flow from oviduct to uterus, and sperm align themselves against the 
flow direction and swim upstream (MIKI AND CLAPHAM, 2013), a phenomenon termed rheotaxis. Although sperm rheotaxis is a passive process dominated by fluid mechanics, these active particles actively adapt to fluid flow (ZHANG et al., 2016). A bending wave is produced during their motion, which propagates along the flagellum; this is a feature independent of the swimming fluid. However, if the spermatozoa fails to move, make little forward progress, moves slowly or exhibit a "non-linear motility", infertility problems may arise (TULSIANI, 2012). The bending waves formation and propagation require adenosine triphosphate (ATP) hydrolysis as the main source of biochemical energy. Therefore, a flagellum represents a typical biological micro-machine, which transforms chemical energy into mechanical energy with high efficiency. In fact, the addition of ATP in nonmotile sperm can reinduce its motion. Thus, spermatozoa that have not been activated in vivo can be activated in vitro by cAMP (a derivative of ATP).

Another interesting application of the study of active matter is that bacteria and other microorganisms can enhace mixing in their natural environments by creating eddies in their wake and by dragging water along. However, these mixing mechanisms are inefficient for microorganisms, because swimming-induced variations in velocity, temperature, and dissolved substances are evened out before they can be advected. In bioconvection, however, microorganisms induce water movement not by propulsion directly but by locally changing the fluid density, which drives convection (SOMMER et al., 2017). Larger animals, such as $C$. elegans can enhace this mixing.

On the other hand, the interaction between microorganisms can also stabilize suspensions. For instance, flagellar entanglements have been shown to stabilize bacterial networks (INGHAM AND BEN JACOB; SERRA et al., 2008; 2013). This is related to the spread and control of microbial infections. One important virulence strategy is bacterial motility. Motile bacteria are able to direct their own movement towards important nutrients or away from harmful substances (WIEDEMANN et al., 2015). Horstmann (HoRstmann, 2017) studied how Salmonella mobility affects its virulence. This bacterium consists of three main parts: the basal body, the hook, and the filament. The basal body is composed of an engine (MotAB) and components that anchor the flagellum to the bacterial membrane. These include rotor and stator protein complexes that are necessary for motor force generation and flagellar rotation (FRANCIS et al.; SANSONETTI, 1993; 2002).

Breakthroughs in materials science and nanofabrication over the last decade have also enabled the design and manufacturing of a multitude of synthetic microswimmers (EBBENS AND Howse, 2010), relying on a variety of propulsion mechanisms that in some cases mimic those found in biology. Some of the most popular mechanisms involve self-diffusiophoresis using nonuni- 
form surface reactions (MORAN AND POSNER, 2017), self-electrophoresis using redox reactions (PAXTON et al., 2004), and bubble generation (GiBBS AND ZHAO, 2009), among others. Although some of these particles are very efficient swimmers, the macroscopic characterization of their rheological properties has been lagging, in part due to sedimentation out of suspension and to the difficulty in concentrating these particles without causing aggregation.

Synthetic nanomotors, based on a multitude of propulsion mechanisms, have been developed over the past decade toward diverse biomedical applications. Major advances in nanomotor technology, including the design of powerful multifunctional machines, advanced motion control and cargo towing capabilities, have facilitated different biomedical applications ranging from cell sorting (BALASUBRAMANIAN et al., 2011), to DNA hybridization (KAGAN et al., 2011). Moreover, the study of biological propulsion can help in the creation of fuel-free micro/nanomachine propulsion mechanisms, including the utilization of magnetic (ZHANG et al.; GosH AND EMMONS; GAO AND WANG; TOTTORI et al.; GAO AND WANG, 2009; 2008; 2014; 2012; 2014), electrical (Chang et al.; Loget And KuHn; CAlvo-MarZal et al., 2007; 2010; 2010), optical (LiU et $a l .$, 2010) or ultrasound (WANG et al.; KAGAN et al.; GARCIA-GRADILlA et al., 2012; 2012; 2013) fields.

Magnetically driven nanomotors, inspired by nature swimming microorganisms, are particularly promising for use in a variety of in vivo biomedical applications. Such micromotors can swim under externally applied magnetic fields in various biofluids, and perform complex maneuvers while obviating fuel requirements. Magnetic actuation is suitable for in vivo applications since the required field strengths are harmless to humans.

\subsection{Rheology of active suspensions}

Rheology - the study of material flow behavior - is traditionally carried out by imposing bulk displacements on a macroscopic sample of material in a viscometer, e.g. via a shearing or extensional flow. When displacements are steady in time, a range of shear strains may be imposed to study flow rate-dependent behaviors. Oscillatory and other time-varying motions may be applied in order to study viscoelastic and transient responses. Constitutive relations between the imposed flow and the stress may then be developed and, from these, material properties such as viscosity may be inferred. 
However, many of the techniques used to understand passive systems are not appropriate to analyze active suspensions. Suspensions of active particles exhibit a rich phenomenology, which is very different from that of passive systems. The mere propulsion of individual microswimmers can result in unusual transport properties such as enhanced diffusion, accumulation near boundaries, and rectification (ELGETTI et al., 2015). As mentioned before, in more concentrated systems, interactions between particles often result in emergent collective dynamics, such as sustained chaotic turbulent-like motions in the bulk (SOKOLOV AND ARANSON, 2009b) and spontaneous unidirectional flows under confinement (WIOLAND et al., 2015). The response of active suspensions to external forcing can defy intuition, with rheological measurements in simple flows reporting a peculiar transition to superfluid-like behavior (LOPEZ et al., 2015)

Biological and chemical contributions can become more (or less) important under variations in, e.g., ionic strength; thus, biologically relevant changes in $\mathrm{pH}$, for example, can lead to profound changes in material properties such as viscosity. Since in these traditional rheological approaches, material perturbations are applied over macroscopic length scales and may therefore exclude important materials of interest, it is necessary to use other techniques to fully describe the materials.

Another key factor in the rheological response of active suspensions, in addition to microswimmer transport properties, is the disturbance flows induced by individual particles. In the case of passive objects, a disturbance velocity only arises if an external force or flow field is imposed; this is unlike self-propelled particles, which drive fluid disturbances even in quiescent environments (SAINTILLAN, 2018).

Furthermore, inertia, secondary flows and viscoelastic waves are very important issues when dealing with biological fluids. Propagating waves may come from either viscous momentum diffusion or elastic shear waves or both for viscoelastic materials in general, even at vanishing Reynolds numbers. For rotational rheometers, surface tension may also result in a torque that should not occur in an ideal, rotationally symmetric geometry. The surface tension torque is reduced by maximizing rotational symmetry of the contact line, minimizing evaporation and the migration of the contact line, reducing the radial location of the contact line, and lowering the surface tension. An alternative approach is to observe the material over microscopic length scales, known as "microrheology". Microrheology comprises a theoretical framework and experimental technique in which the motion of a particle (or set of particles) is tracked and its motion studied to infer the properties of the surrounding medium. 
Active particles are very complex systems. For instance, some of them may exhibit intrinsic viscoelasticity due to intracellular structure and very nonsymmetric shapes. This creates more difficulties on the modelling of the particles and the constitutive equations. Besides, the analysis of the rheological data is very dependent on the used flow. Some frameworks are not adequate for pulsatile flows, which are very commom in vivo.

\subsection{Microrheology}

Microrheology is an emerging technique that probes mechanical response of soft material at micro-scale. Generally, microrheology technique can be divided into active and passive versions. For active microrheology, a user-controlled force, e.g. magnetic force, electrostatic force, optical tweezers etc., is applied to embedded particle in medium of interest, and the particle motion under this force is tracked. For passive microrheology, the embedded particles only move due to thermal fluctuations in the medium, i.e. Brownian motion, and their trajectories are tracked and analyzed (XIA et al., 2018).

As a matter of fact, "microrheology" indicates a family of methods, such as video-particle tracking, light scattering, laser tweezers or magnetic tweezers. For instance, dynamic light scattering (DLS) is the ancestor of today's microrheology. It can be used with or without the addition of tracer scattering particles. Requiring more than $90 \%$ of light to be transmitted unscattered, this technique is limited to quite transparent samples, in order to avoid the complication of multiple scattering (BERNE AND PECORA, 1976). In opaque systems, diffusive wave spectroscopy (DWS) can be used. This method has the additional advantage of extending measurements to very high frequencies and very good spatial resolution. However it is still a bulk technique, with the limitations of large (milliliter) sample sizes and inability to resolve spatial heterogeneity (BROWN, 1993).

One of the most common method is video particle tracking, which allows to measure the compliance and can yield a complete characterization of the linear viscoelasticity of the matrix. The most challenging aspects of a video tracking experiment are often the process of acquiring the trajectory of a number of particles and the image analysis (TANAKA et al., 1973), which consist in: analyze individual frames to extract the coordinates of all the particles in the frame and then match the particles through subsequent frames to produce data of trajectories. 
Another commom method is two-particle correlation. The particle-tracking approach described above assumes that the probe particles do not affect the system locally. This is not always the case. The probe particle in a solution could become surrounded by either a depletion layer or a more dense layer of molecules. If this is the case, it is necessary to image and calculate the fluctuations as a function of the distance between pairs of particles. These 2-particle correlations are not affected by the local environment around each bead, and provide instead an unbiased probe of the response of the bulk matrix. However, one important downside of 2-particle correlation vs. singleparticle, is that a much more extensive amount of video data needs to be recorded and analyzed in order to have good statistics for many pairs of particles at many distances.

Overall, microreology has several advantages over macroreology: an extremely wide frequency range, it is a local probe, ideally suitedto heterogeneous systems, the sample can have very low viscosity and very low elasticity and it is applicable to non-conventional geometries. On the other hand, it is still quite difficult to make use of microrheology to study the nonlinear response, it is limited to materials that are at least partially transparent to light, is computationally intensive and, for very stiff or viscous materials, it will be challenging to observe the very small motion of the probe particles (CICUTA AND DONALD, 2007).

\subsection{General objetives}

The main contribution of this thesis is the development of a framework to analyze active (and living) suspensions that can be extended to any type of microorganism or molecule. In general, it presents a complete picture of how to analyze a suspension of active (or passive) particles. Figure 1.4 presents the main contributions of the thesis according to the expected objectives.

Numerous experiments have focused on the dynamics in suspensions of swimming bacteria, but almost none focused on nematodes. Moreover, until now there is no fixed procedure to completely analyze and model an active suspension.

In the present study we propose a complete methodology for the analysis of an active nematode suspension. First, in chapters 3 and 4, respectively, we investigate the kinematics and rheological properties of $C$. elegans immersed in a Newtonian fluid, wherein the activity of the microorganism is observed. This study aims to determine the viscometric characteristics based on 
Figure 1.4: Main contributions of the thesis. [Source: the author.]

\section{Thesis main contributions}

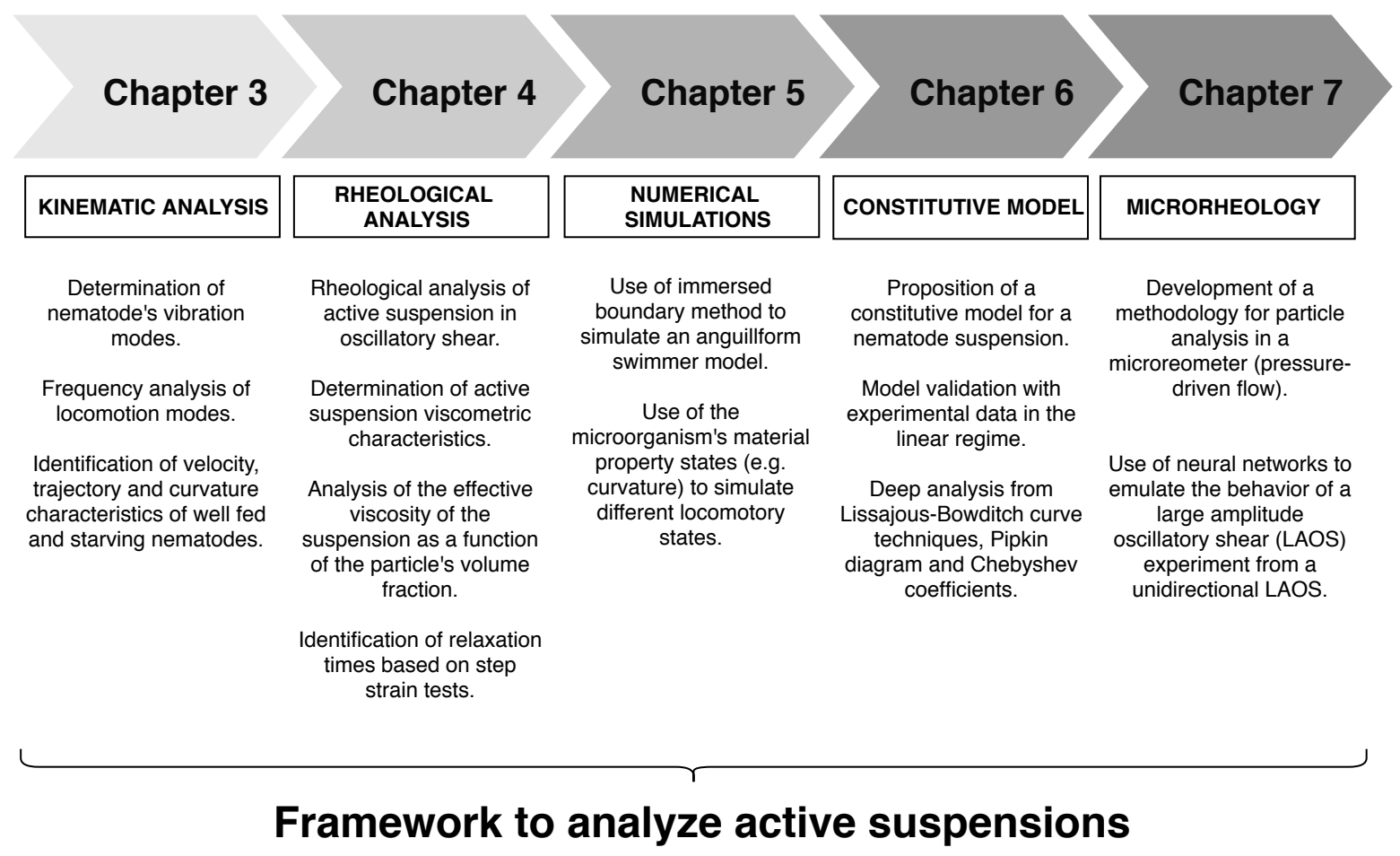

the kinematic analysis performed in oscillatory shear and step strain tests.

After analyzing the kinematics of the microorganism and the rheology of the suspension, it is possible to propose some simplifications for the modeling of the intrinsically elastic behavior of the nematode. In order to validate the propositions immersed boundary simulations are performed and the results are compared with experimental kinematic data in chapter 5. These simplifications are extremely important for a rheological model to be put forward in chapter 6 . The proposition of this constitutive model allows tests in several geometries and parameters to be performed. To do so, the model is validated using the experimental rheological results in linear viscoelasticity and, 
afterwards, tested considering the non-linearities.

To conclude, in chapter 7 another framework based on microreology is presented in such a way that the instabilities related to the microscopic behavior of the microorganism can be studied. For this, different tools of signal analysis and artificial intelligence are used to emulate data based on experimental results obtained using in vivo inspired flows.

The major contribution of the study concerns the observation, characterization, modeling and simulation of a microsized animal that moves in different fashion, depending on the environment, and the surrounding fluid. The rheological properties analyzed, simuations performed and model proposed can be used for both production of artifitial microorganisms and control of living organisms. Moreover, this combination of analyses and techniques can be used to study any type of passive and active suspension providing new and conclusive results regarding the rheological characterization and the physical behavior of the particles. 


\section{Theoretical Foundations}

In this chapter, we intend to introduce the fundamental theory necessary to understand the rheological properties of our suspensions and the equations that model the flow in any continuous material. Although many constitutive models are phenomenological, there are some rules to propose the correct hypotheses. These principia are part of what is called constitutive formalism. Many properties of a fluid when observed in a suficiently small scale, which allows us to analyze the molecular behavior, present a strong non-linear distribution. However, between the molecular scale and macroscale there is the continuum local scale, defined based on a suficiently small volume so it can be considered local regarding the macroscale and large enough to contain a substantial number of molecules. This allow us to assume a perfecly homogeneous distribution of the microscopic structure and therefore of its physical properties such as mass.

Batchelor (BATCHELOR, 1977) highlights that in the continuum scale, the measured properties are the analyzed volume average of the molecular effects. Based on that, we may observe that the molecular fluctuations do not affect these properties, which remain constant. However, in this work, mesoscale fluctuations must be considered due to the motion of the active particles. The relation between stress and deformation is modeled using constitutive equations, which represent the continuum scale of the fuids.

\subsection{Constitutive Formalism}

Simple rheological equations that describe non-linear viscoelastic phenomena in polymeric liquids have long attracted the attention of many rheologists. Although there are many ways of deriving such equations, all constitutive equations must follow some principles (TRUESDELL, 1991) in order to be considered adequate. In what follows, the most important of them are presented.

\subsubsection{Casuality principle}

The casuality principle states that the stress tensor depends on the recent history of the velocity field or deformation. Under a general perspective, we may say that a constitutive equation 
relates the stress of the material, $\sigma(t)$, in $t-\delta t$ with $t$. Therefore, the stress tensor is a function of integrals and derivatives of the velocity field with respect to time and space and contain coefficients related to the physical properties of the material.

For a linear isotropic viscoelastic material the stress-strain relation is given by Boltzmann superposition principle, which describes the response of a material to different loading histories (SHUKLA AND M., 2017), and can be represented either in a stress-controlled or a strain-controlled form, respectively, given by

$$
\gamma(t)=\int_{-\infty}^{t} J\left(t-t^{\prime}\right) \dot{\sigma}\left(t^{\prime}\right) d t^{\prime}
$$

and

$$
\sigma(t)=\int_{\infty}^{t} G\left(t-t^{\prime}\right) \dot{\gamma}\left(t^{\prime}\right) d t^{\prime}
$$

where $\sigma(t)$ is the stress, $\dot{\gamma}\left(t^{\prime}\right)$ is the strain-rate, $t$ is the time, and $t^{\prime}$ is the time at which the deformation was imposed. The response functions, respectivaly, the creep compliance, $J$, and relaxation modulus, $G$, solely depend upon the time elapsed since application of deformation field $\left(t-t^{\prime}\right)$. Thus the stress at a given time $t$ is determined by the entire history of the strain. The upper integration limit ensures the causality. Mathematically, this integral, also called the hereditary integral, represents a time convolution of the relaxation function and strain rate. If we use the symbol $*$ for the convolution, then equation 2.2 can be written as

$$
\sigma(t)=G(t) * \dot{\gamma}(t)
$$

\subsubsection{Local action principle}

This principle postulates that only near particles must be involved into the determination of the stress at a specific location of a control volume. It is directly related to the idea of near-field 
forces between particles and molecules. Consider a Lagrangian description of a system, where a material body $\Upsilon$ located in a initial position $\boldsymbol{x}$ at $t=0$, moves itself to a position $\boldsymbol{X}^{\prime}\left(\boldsymbol{x}, t^{\prime}\right)$ and them to $\boldsymbol{X}^{\prime \prime}\left(\boldsymbol{x}, t^{\prime \prime}\right)$ at $t^{\prime \prime}$. At an specific location $O$ at $t^{\prime}$, we have by Taylor expansion:

$$
\boldsymbol{X}^{\prime}(\boldsymbol{x}+d \boldsymbol{x})=\boldsymbol{X}^{\prime}(\boldsymbol{x})+\frac{\partial \boldsymbol{X}^{\prime}}{\partial \boldsymbol{x}} d \boldsymbol{x}+\mathcal{O}|d \boldsymbol{x}|^{2}
$$

so

$$
d \boldsymbol{X}^{\prime}=\boldsymbol{X}^{\prime}(\boldsymbol{x}+d \boldsymbol{x})-\boldsymbol{X}^{\prime}(\boldsymbol{x})=\frac{\partial \boldsymbol{X}^{\prime}}{\partial \boldsymbol{x}} d \boldsymbol{x}+\mathcal{O}|d \boldsymbol{x}|^{2}
$$

This analysis is based on the continuum hypotheses. Thus, the scale $\left|d \boldsymbol{X}^{\prime}\right|$ must be way bigger than the molecular $\left(\lambda^{\prime}\right)$ and smaller than the macroscopic scale $(L)$. Thus

$$
d \boldsymbol{X}^{\prime}=\frac{\partial \boldsymbol{X}^{\prime}}{\partial \boldsymbol{x}} d \boldsymbol{x} \text { at } t=t^{\prime}
$$

and

$$
d \boldsymbol{X}^{\prime \prime}=\frac{\partial \boldsymbol{X}^{\prime \prime}}{\partial \boldsymbol{x}} d \boldsymbol{x} \text { at } t=t^{\prime \prime}
$$

Neglecting the second order terms, we have and defining the deformation tensor as $\boldsymbol{F}(t)=$ $\partial \boldsymbol{X}^{\prime} / d \boldsymbol{x}$, we may write the stress as a functional namelly

$$
\boldsymbol{\sigma}(t)=\mathcal{F}\left\{\boldsymbol{F}\left(t^{\prime}\right)\right\}_{0<t^{\prime}<t^{\prime \prime}}
$$

Thus, the stress tensor is a function of the deformation tensor. 


\subsubsection{Material Frame Indifference principle}

A constitutive equation must be valid regardless the coordinate system. Therefore, the function $\boldsymbol{F}$ must be mantained independent of the stress tensor $\boldsymbol{\sigma}$ and the velocity gradient $\nabla \boldsymbol{u}$. Physically, this principle implies that a change in coordinates should not lead to new constitutive equations.

Mathematically, the principle of material frame-indifference can be stated as invariance of constitutive function under change of frame. However, great care must be take regarding what such constitutive functions are. They should not simply be the constitutive functions relative to some reference configuration in two different frames, because a choice of reference configuration may change material properties (LIU, 2004).

The indifference principle postulates that if a constitutive equation is satisfied by a given process, characterized by a movement (successive changes of configuration) and a symmetrical tensor, denoted respectively by:

$$
\boldsymbol{x}=\boldsymbol{x}(X, t) \text { and } \boldsymbol{\sigma}=\boldsymbol{\sigma}(X, t),
$$

where $X$ is the label of a given material particle and $t$ is the time, then this must also be satisfied by an equivalent process $\left(\boldsymbol{x}^{*}, \boldsymbol{\sigma}^{*}\right)$, where the tensile motion and tensor are given by:

$$
\begin{gathered}
\boldsymbol{x}^{*}=\boldsymbol{x}^{*}\left(X, t^{*}\right)=\boldsymbol{x}(t)+\boldsymbol{Q}(t) \boldsymbol{x}(X, t), \\
\boldsymbol{\sigma}^{*}=\boldsymbol{\sigma}^{*}\left(X, t^{*}\right)=\boldsymbol{\sigma}(t)+\boldsymbol{Q}(t) \boldsymbol{\sigma}(X, t) \boldsymbol{Q}(t)^{T}, \\
t^{\prime}=t-a,
\end{gathered}
$$


where $\boldsymbol{Q}(t)$ is a ortogonal time-dependent tensor related to a rigid body rotation and $a$ an arbitrary constant. The physical basis of this principle lies in the fact that the material must be independent of the referential. That is, the instantaneous field of stresses (response of the material) must be independent of the movement of the observer. In other words, the mechanical response of a material must be invariant in relation to a movement of an arbitrary rigid body.

It is interesting to notice that this principle must be applied with care. Material Frame Indifference applies, in a strong approximate sense, to most areas of continuum mechanics where there is a clear cut separation of scales so that the ratio of fluctuating to mean time scales is extremely small. While it breaks down for the three-dimensional case, it rigorously applies to Reynolds stress models in the limit of two-dimensional turbulence where an analogy is made between the Reynolds stress tensor and the non-Newtonian part of the stress tensor in the laminar flow of a non-Newtonian fluid. On the other hand, the general invariance group of constitutive equations that is universally valid is the extended Galilean group of transformations which includes arbitrary time-dependent translations of the spatial frame of reference; rotational frame-dependence then enters exclusively through the intrinsic spin tensor (FAUCI AND DILLON, 1998).

\subsubsection{Fading Memory}

The Fading Memory principle postulates that deformations that occured in a long past should have less influence on the determination of the tension (TRUESDELL, 1966). That is, the instantaneous structure of the stress field is more closely related to more recent events (deformations). In other words, being $t^{\prime}$ a instant of time in the past and $t$ the present time, the deformation effects on the stress tensor $\sigma$ is greater for $t$ than $t^{\prime}$.

In the case of elastic materials, the time of rest has no influence on its behavior after releasing stresses. Such materials do not forget the pre-history of their deformations at all. However, the behavior of many materials is intermediate. It is possible to observe a phenomenon of retarding elastic recoil by increasing the delay time, i.e. it is reasonable to treat this effect in terms of the fading memory of the pre-history of their deformations. The reasons are quite evident: in the period preceding the elastic recoil, a relaxation partly takes place and stresses responsible for the posteffect decrease. 
The first illustration of the competition between memory effects and nonlinear elastic response in materials with fading memory was provided by the theory of propagation of acceleration waves. It is tempting to conjecture that the dissipative effect of viscosity dominates in motions near equilibrium, preserving the smoothness of such motions, while in motions far from equilibrium the destabilizing action of nonlinear instantaneous elastic response may overpower the dissipative effect of viscosity causing a sudden variation of the acceleration and the velocity gradient.

\subsection{Constitutive Models}

The most simple constitutive relation between shear stress and shear rate was proposed by Newton (LANDAU AND LifSHITZ, 1998) and describes the behavior of the so called Newtonian fluids. This relationship is given by

$$
\boldsymbol{\sigma}=\boldsymbol{\eta}: \dot{\gamma}
$$

where $\boldsymbol{\eta}$ is the viscosity tensor that maps the strain rate tensor onto the viscous stress tensor. However, due to spatial symmetries the 81 viscosity coefficients are not all independent. For instance, for isotropic Newtonian fluids, the coefficients can be reduced to 2 independent parameters: dynamic viscosity $\mu$ and bulk viscosity $\kappa$, and the latter does not play an important role in incompressible flows.

For many fluids, the Newtonian constitutive equation does not make accurate predictions. For materials that are not pure (suspensions, emulsions), for large molecule liquids (polymers, gels), and for liquids with special intermolecular forces (ionic liquids, magnetic liquids), the Newtonian constitutive equation is inadequate. Materials that generate velocity and stress effects that are not represented by the Newtonian constitutive equation are called non-Newtonian fluids.

Indeed, under appropriate circumstances, the apparent viscosity of certain materials is not only a function of flow conditions (geometry, rate of shear), but it also depends on the kinematic history of the fluid element under consideration. It is convenient to group such materials into three categories. 
The first one is known as purely viscous, inelastic, time-independent or generalized Newtonian fluids. The value of $\dot{\gamma}$ of these systems is determined only by the current value of $\boldsymbol{\sigma}$ at that point. When the relation between $\sigma$ and the shear rate shows further dependence on the duration of shearing and kinematic history, the fluids are called time-dependent. And when the system exhibits a blend of viscous fluid and solid-like behavior, showing partial elastic recovery, recoil and creep, it is called viscoelastic.

As noted earlier, the aforementioned classification scheme is quite arbitrary, though convenient, because most real materials often display a combination of two or even all these types of features under appropriate circumstances. In this thesis, we will focus on viscoelastic fluids due to the intrinsic elasticity of the microorganisms.

Viscoelastic fluids are materials that exhibit both viscous and elastic responses to forces. The distinction between viscous and elastic materials is best illustrated by their responses to a sudden deformation: stresses created in an elastic material stay constant in time for as long as the deformation is present, while stresses in a viscous fluid dissipate on a time scale governed by its viscosity. For example, a bow is stressed as long as it is strung by a bowstring, while in spilled water all stresses disappear once the fluid comes to rest. Essentially, whether a material is fluid-like or solid-like is determined by its longtime response to a deformation. There are basically two main ideas of how to include memory, attributed to Boltzmann and Maxwell. Boltzmann's model is an integral equation for the stress tensor, assuming that it depends linearly on the symmetric part of the strain rate tensor $\boldsymbol{D}(\boldsymbol{u}(\boldsymbol{x}, t))$ :

$$
\boldsymbol{\sigma}(t, x)=2 \int_{-\infty}^{t} G(t-s) \boldsymbol{D}(\boldsymbol{u}(\boldsymbol{t}, \boldsymbol{x})) d s
$$

Here, $G(t-s)$ is called the stress relaxation modulus and it should be positive and monotonic. To recover the Newtonian model, $G$ must be a multiple of the delta function. On the other hand, Maxwell theory of viscoelasticity assumes that the stress is linked to the velocity gradient by an ordinary differential equation such as

$$
\boldsymbol{\sigma}+\lambda^{\prime} \frac{\partial \boldsymbol{\sigma}}{\partial t}=\eta \frac{\partial \boldsymbol{\gamma}}{\partial t}
$$


where $\dot{\gamma}=\partial \gamma / \partial t=2 \boldsymbol{D}$, being $\boldsymbol{D}$ the strain rate tensor and $\lambda^{\prime}=\eta / G$ a material constant (time scale). Multiplying this expression by an integrating factor, namely $e^{\left(1 / \lambda^{\prime}\right)}$, we obtain the constitutive equation for the stress tensor

$$
\boldsymbol{\sigma}(t)=\frac{2 \eta}{\lambda^{\prime}} \int_{-\infty}^{t} e^{-\left(t-t^{\prime}\right) / \lambda^{\prime}} \boldsymbol{D}\left(t^{\prime}\right) d t^{\prime}
$$

In this simple case, the elastic component is related to $G^{\prime}$, while the damping effect is connected to $\mu$. The shear is then given by

$$
\dot{\gamma}=\frac{\dot{\sigma}_{E}}{G}+\frac{\sigma_{V}}{\mu}=\frac{\dot{\sigma}}{G}+\frac{\sigma}{\mu},
$$

where $\dot{\sigma}_{E}$ is related to the Hookean spring and $\sigma_{V}$ to the damper.

In reality, however, Maxwell model is insufficient to describe even the linear rheology of viscoelastic fluids. Nevertheless, it is a very useful minimal model that sets the stage for more complete theories. Equation 2.15 suffers from a serious physical problem: it is not frame-invariant. The simplest equations that take the relaxation of the stress into account are produced by writing a frame-invariant analogue of the linear Maxwell model already discussed. By choosing either the upper or lower-convective derivative for the full-time derivative in equation 2.15 we arrive at:

$$
\boldsymbol{\tau}=-p \boldsymbol{I}+\boldsymbol{\sigma}
$$

where $\tau$ is the total stress and the non-Newtonian contribution to the stress $\sigma$ obeys

$$
\boldsymbol{\sigma}+\lambda^{\prime} \stackrel{\nabla}{\boldsymbol{\sigma}}=\eta_{p} \boldsymbol{D} \quad \text { (upper-convected Maxwell or UCM) }
$$

as the upper-convected or 


$$
\boldsymbol{\sigma}+\lambda^{\prime} \stackrel{\Delta}{\boldsymbol{\sigma}}=\eta_{p} \boldsymbol{D} \quad \text { (lower-convected Maxwell or LCM) }
$$

as the lower-convected. Here, $\eta_{p}$ is the polymer contribution to the viscosity and $\stackrel{\Delta}{\sigma}$ and $\stackrel{\nabla}{\sigma}$ are defined respectively as

$$
\stackrel{\Delta}{\sigma}=\frac{D \boldsymbol{\sigma}}{D t}+\boldsymbol{\sigma} \cdot \nabla \boldsymbol{u}+(\nabla \boldsymbol{u})^{T} \cdot \boldsymbol{\sigma}
$$

and

$$
\stackrel{\nabla}{\boldsymbol{\sigma}}=\frac{D \boldsymbol{\sigma}}{D t}-\nabla \boldsymbol{u} \cdot \boldsymbol{\sigma}-\boldsymbol{\sigma} \cdot(\nabla \boldsymbol{u})^{T}
$$

Alternatively, one can use a linear combination of the upper and lower-convected derivatives in the Maxwell model to obtain the Johnson-Segalman equation:

$$
\boldsymbol{\sigma}+\lambda^{\prime}\left(\frac{1+a}{2} \underset{\boldsymbol{\sigma}}{\nabla}+\frac{1-a}{2} \stackrel{\Delta}{\boldsymbol{\sigma}}\right)=\eta_{p} \boldsymbol{D}
$$

The slip parameter $a$ sets the relative importance of the two objective time derivatives. If $\sigma$ obeys the UCM model, the resulting set of equations is called the Oldroyd-B model (OLDROYD, 1950). This model is often formulated in terms of the total deviatoric stress, which satisfies

$$
\boldsymbol{\sigma}+\lambda^{\prime} \stackrel{\nabla}{\boldsymbol{\sigma}}=\eta\left(\boldsymbol{D}+\lambda_{r}^{\prime} \stackrel{\nabla}{\boldsymbol{D}}\right) \quad(\text { Oldroyd-B })
$$

where $\eta$ is the summation of the polymeric viscosity $\eta_{p}$ and the solvent viscosity $\eta_{s}$. The so-called retardation time $\lambda_{r}^{\prime}$ is not an independent time scale, but is in fact a combination of the Maxwell relaxation time $\lambda^{\prime}$ and the solvent and polymeric viscosities $\lambda_{r}^{\prime}=\lambda^{\prime}\left(\eta_{s} / \eta\right)$. One drawback of 
the viscoelastic models above is that tensile stresses can grow without bound in extensional flows. Another class of models is then formed by adding nonlinear terms in the UCM model. One example of such models is the Giesekus equation (GIESEKUS; GIESEKUS, 1982; 1985)

$$
\boldsymbol{\sigma}+\lambda^{\prime} \stackrel{\nabla}{\boldsymbol{\sigma}}+\alpha \frac{\lambda^{\prime}}{\eta_{p}} \boldsymbol{\sigma} \cdot \boldsymbol{\sigma}=\eta_{p} \boldsymbol{D} \quad \text { (Giesekus) }
$$

Here $\alpha$ is a dimensionless model parameter that should be kept smaller than $1 / 2$ to avoid a nonmonotonic dependence of the shear stress on the shear rate in simple shear flows. The origin of the term involving $\alpha$ can be associated with Brownian motion and/or anisotropic hydrodynamic drag on the constituent polymer. Another example is given by the Phan-Thien-Tanner (PTT) model (Phan-ThiEn AND TANner; Phan-ThiEn AND TANNER, 1978; 1977)

$$
f(\boldsymbol{\sigma})+\lambda^{\prime} \stackrel{\nabla}{\boldsymbol{\sigma}}=\eta_{p} \boldsymbol{D} \quad(\text { Phan-Thien-Tanner })
$$

where $f(\boldsymbol{\sigma})$ is a nonlinear function that can be chosen either in its exponential or, more commonly, in its linear form if $\operatorname{tr}(\boldsymbol{\sigma})$ is small enough.

$$
f=\exp \left[-\varepsilon\left(\frac{\lambda^{\prime}}{\eta_{p}} \operatorname{tr}(\boldsymbol{\sigma})\right)\right] \sim 1-\varepsilon\left(\frac{\lambda^{\prime}}{\eta_{p}}\right) \operatorname{tr}(\boldsymbol{\sigma})
$$

In this case, the parameter $\varepsilon$ is related to the elongational behaviour of the fluid, precluding the possibility of an infinite elongational viscosity in a simple stretching flow as would occur for an upper Maxwell model, UCM, in which $\varepsilon=0$. It basically controls how fast the effective polymeric viscosity and the relaxation time decrease with the stress. Other constitutive relations were developed to correct the unphysical behavior in the Oldroyd-B and similar models, the so-called finite-extensibility-nonlinear-elastic (FENE) models.

The two most commonly used models of this type are the FENE-CR model, suggested by 
(ChilcotT AND RALlison, 1988) and the FENE-P model (a Gaussian closure of the kinetic theory model suggested by Peterlin (BIRD et al., 1980)):

$$
\begin{gathered}
f(\boldsymbol{\sigma})+\lambda^{\prime}\left(\frac{\nabla}{\boldsymbol{\sigma}}\right)=\eta_{p} \boldsymbol{D} \quad(\text { FENE-CR }) \\
f(\boldsymbol{\sigma})+\lambda^{\prime}\left(\frac{\nabla}{f(\boldsymbol{\sigma})}\right)=\frac{\eta_{p}}{f(\boldsymbol{\sigma})} \boldsymbol{D}-\eta_{p} \frac{D}{D t}\left(\frac{1}{f(\boldsymbol{\sigma})}\right) \boldsymbol{I} \quad(\text { FENE-P) }
\end{gathered}
$$

In these models, the function $f$ is given by

$$
f(\boldsymbol{\sigma})=1+\frac{\lambda^{\prime}}{\eta_{p} L_{f}^{2}} \operatorname{tr}(\boldsymbol{\sigma}),
$$

being $L$ a dimensionless parameter related to the maximum possible extension of the polymer chains. There are several versions of these models in the literature, but in the limit of large $L$ they all reduce to the above equations.

\subsection{Rheological Flows}

The description of a suspension or a polymeric fluid differs from that provided by Newton's viscosity law. A Newtonian, incompressible fluid at constant temperature is completely characterized by two material constants: density and viscosity. Therefore, the distribution of stress and velocity are fixed for any flow type. The situation becomes more complex for non-Newtonian fluids. First of all, they do not present constant viscosity and may have normal stresses even in incompressible flows and elastic effects. Although different types of experiments applied to Newtonian fluids lead a single material constant (viscosity), the same applied to a non-Newtonian liquid leads to a set of material functions, which depend on the shear rate, frequency, time, and other parameters. These 
functions aim to promote the classification of the different types of non-Newtonian fluids and are essential for the determination of constants of specific constitutive equations.

Newtonian fluids under steady plane flow (simple shear) present $\sigma_{x x}=\sigma_{y y}=0$. However, for non-Newtonian fluids, in the absence of a constitutive equation, we must consider that the six components of the stress tensor are different from zero. Since we cannot separate the contributions of pressure and traction, the only quantities that we usually analyse experimentally are the shear stress $\sigma_{x y}$ and both normal stress differences $N_{1}=\sigma_{x x}-\sigma_{y y}$ and $N_{2}=\sigma_{y y}-\sigma_{z z}$.

\subsubsection{Small Amplitude Oscillatory Shear (SAOS)}

The viscoelastic properties of non-Newtonian fluids with memory can be determined by experiments with low-amplitude oscillatory shear, evaluating the viscoelastic response of the fluid given a known external excitation. The system oscillates with a given frequency $\omega$ and, considering a regime of small deformations, and therefore linear, it is assumed that the shear stress oscillates with the same frequency of the system, but not necessarily in phase. Consider, therefore, a simple oscillating shear with $u_{x}=\dot{\gamma}_{0} \cos (\omega t) y$, being $\dot{\gamma}_{0}$ the amplitude of the shear rate (a positive and real value). We consider the application of an excitation to the system in the form of an oscillatory shear deformation $\gamma(t)=\gamma_{0} \sin (\omega t)$, where $\gamma_{0}$ is the deformation amplitude.

The excitation response imposed to the system will be represented by a shear stress $\sigma$, written in terms of deformation amplitude $\gamma_{0}$ and the deformation itself $\gamma(t)$. We may write it in terms of sums of sines and cosines to make explicit the components of in-phase and out-of-phase with the excitation:

$$
\sigma(t)=G^{\prime}(\omega) \gamma_{0} \sin (\omega t)+G^{\prime \prime}(\omega) \gamma_{0} \cos (\omega t)
$$

or

$$
\sigma(t)=\eta^{\prime}(\omega) \dot{\gamma}_{0} \cos (\omega t)+\eta^{\prime \prime}(\omega) \dot{\gamma}_{0} \sin (\omega t)
$$


being $G^{\prime}, G^{\prime \prime}, \eta^{\prime}$ and $\eta^{\prime \prime}$ viscoelastic functions of the material. We must note that for a perfect elastic solid, we have $G^{\prime \prime}(\omega)=0$ and $\sigma=G^{\prime} \gamma(t)$, where $G^{\prime}(\omega)$ is known as the elastic shear modulus. For a pure Newtonian fluid, $\eta^{\prime \prime}(\omega)=0$ and $\sigma(t)=\eta^{\prime}(\omega) \dot{\gamma}(t)$. The viscoelastic parameters present the following physical interpretations: $G^{\prime}$, designated storage modulus, is associated with the elastic character of the fluid, i.e. the energy stored during the deformation. $G^{\prime \prime}$ is referred to as the loss modulus and is associated with the fluid viscous character and its energy dissipation. Therefore $\eta^{\prime}$ is called dynamic viscosity and it is also related to the dissipative effects, while $\eta^{\prime \prime}$ represents the imaginary part of the complex viscosity, being associated with the intrinsic elasticity of the fluid; Another important parameter of the system is $\tan (\delta)=G^{\prime \prime}(\omega) / G^{\prime}(\omega)$ since it is a measure of the system damping capacity.

Another way to approach this problem is through the use of complex variables, in which the excitation imposed on the system oscillating with a frequency $\omega$, is described in the following form

$$
\gamma(t)=\gamma_{0} e^{i \omega t}
$$

Based on that, the stress is given by

$$
\sigma(t)=\tilde{\sigma} e^{i(\omega t+\phi)}
$$

We can then write the shear stress as

$$
\sigma^{*}(t)=G^{*}(\omega) \gamma(t)=\gamma_{0} G^{*}(\omega) e^{i \omega t}
$$

or

$$
\sigma^{*}(t)=\eta^{*}(\omega) \dot{\gamma}(t)=\eta^{*}(\omega) \dot{\gamma}_{0} e^{i \omega t}
$$

So we may say that $G^{*}(\omega)=i \omega \eta^{*}(\omega)$. Since $G^{*}(\omega)=G^{\prime}(\omega)+i G^{\prime \prime}(\omega)$ and $\eta^{*}(\omega)=\eta^{\prime}(\omega)-i \eta^{\prime \prime}(\omega)$, 
we have $G^{\prime}(\omega)=\omega \eta^{\prime \prime}(\omega)$ and $G^{\prime \prime}(\omega)=i \omega \eta^{\prime}(\omega)$.

The interesting fact about the linear regime is that several characteristics of the fluid can be estimated. For instance, when applicable, the shear viscosity can be estimated by applying the CoxMerz rule (COX AND MERZ, 1958). It predicts that the aparent viscosity $\eta$ equals the magnitude of the complex viscosity $\left|\eta^{*}\right|$ for corresponding values of frequency $\omega$ and shear rate $\dot{\gamma}$ for the linear viscoelasticity regime. Laun's (LAUN, 1986) empirical rule also estimates the fist normal stress difference in steady shear, $N_{1}$, from dynamic mechanical data:

$$
N_{1}(\dot{\gamma})=2 G^{\prime}(\omega)=\left[1+\left(\frac{G^{\prime}(\omega)}{G^{\prime \prime}(\omega)}\right)^{1}\right]^{0.7}
$$

Moreover, when $\omega \rightarrow 0$, the Cox-Merz rule reduces to $\eta^{\prime}(\omega)=\eta_{0}=\mu$. This rule also appies for the elastic characteristics of the fluid. In this case, it relates the shear elastic modulus $G^{\prime}(\omega)$ obtained during oscillatory shear test and the first normal stress difference $N_{1}$ so that $2 G^{\prime}(\omega)=$ $N_{1}(\dot{\gamma})$, because (AL-HADITHIH et al., 1992):

$$
\frac{G^{\prime}(\omega)}{\omega^{2}}=\frac{N_{1}(\dot{\gamma})}{2 \dot{\gamma}^{2}}
$$

However, we must notice that this is only true for small excitation frequencies (or shear rates) and suspensions with low elasticity. As the oscillatory frequency increases $N_{1} \neq G^{\prime}$. The applicability of the Cox-Merz rule has already been reviewed in detail (AL-HADITHI et al., 1992b). It has been found to hold for almost all polymer melts, and concentrated and semi-dilute solutions. Deviations from the rule occur at high frequencies, and the oscillatory data can either over or under estimate the steady state data in these cases.

\subsubsection{Step Strain Tests}

The step strain is a viscometric flow used to characterize the memory effects of a complex fluid. Stress relaxation experiments apply a step strain deformation to create an instantaneous strain 
and monitor the stress decay as the specimen is held over time in the same constrained state. The relaxation function $G(t)$ and the relaxation times $\tau_{i}$ can be obtained through this test. A fluid with memory is set between two paralel plates at $t<t_{0}$, being $t_{0}$ a reference time. When $t=t_{0}$, the superior plate is sheared and the fluid is deformed. The excitation of the system, given by the applied shear rate $\dot{\gamma}$ in a short period of time $t_{0}-\left(t_{0}-\epsilon\right)$ can be understood as an impulse function:

$$
\dot{\gamma}(t)=\frac{\gamma_{0}}{t_{0}-\left(t_{0}-\epsilon\right)}=\frac{\gamma_{0}}{\epsilon} .
$$

If we want to obtain the expression for the stress tensor $\sigma(t)$ in the linear viscoelasticity regime, we have:

$$
\sigma(t)=\int_{-\infty}^{t_{0}-\epsilon} G\left(t-t^{\prime}\right) \dot{\gamma}\left(t^{\prime}\right) d t^{\prime}+\int_{t_{0}-\epsilon}^{t_{0}} G\left(t-t^{\prime}\right) \dot{\gamma}\left(t^{\prime}\right) d t^{\prime}+\int_{t_{0}}^{\infty} G\left(t-t^{\prime}\right) \dot{\gamma}\left(t^{\prime}\right) d t^{\prime}
$$

but when $t^{\prime} \in t<t_{0}-\epsilon$ or $t \rightarrow \infty, \dot{\gamma}\left(t^{\prime}\right)=0$ so it reduces to

$$
\sigma(t)=\int_{t_{0}-\epsilon}^{t_{0}} G\left(t-t^{\prime}\right) \dot{\gamma}\left(t^{\prime}\right) d t^{\prime}=\frac{\gamma_{0}}{\epsilon} \int_{t_{0}-\epsilon}^{t_{0}} G\left(t-t^{\prime}\right) d t^{\prime}
$$

Taking the limit when $\epsilon \rightarrow 0$ and applying L'Hôpital's rule we have:

$$
\sigma(t)=\lim _{\epsilon \rightarrow 0} \gamma_{0}\left[\frac{\frac{d}{d \epsilon} \int_{t_{0}-\epsilon}^{t_{0}} G\left(t-t^{\prime}\right) d t^{\prime}}{\frac{d}{d \epsilon} \epsilon}\right]
$$

Therefore, the stress tensor for a viscoelastic fluid subjected to a step strain is given by $\sigma(t)=$ $\gamma_{0} G\left(t-t_{0}\right)$. In other words, the stress tensor of a viscoelastic fluid is not instantaneous. Its stresses respond with an especific delay regarding the applied deformation. Besides, it allows us obtain the relaxation times. 


\subsubsection{Large Amplitude Oscillatory Shear (LAOS)}

Under small amplitude oscillatory shear (SAOS), a material can be characterized by the linear viscoelastic moduli. However, these conventional viscoelastic moduli are not uniquely defined once the material response becomes nonlinear at sufficiently large strains (EWOLDT et al., 2008a). Interpretation of large amplitude oscillatory shear (LAOS) nonlinearities is difficult, and has been hindered because an appropriate and comprehensive framework does not yet exist. The most common method of quantifying LAOS tests is Fourier transform (FT) rheology. However, graphic interpretations and other polinomials decompositions may also be used.

In addition, the nonlinear rheological response in LAOS can be correlated with the microstructure of the viscoelastic sample. In strain-controlled LAOS tests, the imposed strain follows a sinusoidal evolution in time and the corresponding shear stress response measured is not necessarily sinusoidal, revealing the nonlinear behavior of the sample. The sinusoidal strain input is given by:

$$
\gamma(t)=\gamma \sin (\omega t),
$$

where $\omega$ is the frequency, $t$ is the time and $\gamma$ is the maximum strain deformation of the cycle. The results obtained from LAOS can be analyzed using different approaches: from frequency spectrum to Chebyshev decomposition. Some of these frameworks will be presented in the next sections. Figure 2.1 shows the difference of $\sigma(t)$ when under SAOS or LAOS. It is clear that under large amplitude oscillatory shear, the Giesekus model behaves in a very nonlinear fashion. 
Figure 2.1: Stress $[\mathrm{Pa}]$ as a function of time $[\mathrm{s}]$ for Giesekus model (eq. 2.25) with parameters $\omega=0.5, \alpha=0.5, \eta=0.1, \lambda^{\prime}=2$. The dashed line represents $\dot{\gamma}=2.2$ and the solid line represents $\dot{\gamma}=0.2$. The plot bellow shows the frequency $[\mathrm{rad} \cdot \mathrm{s}]$ spectrum of both signals. [Source: the author.]
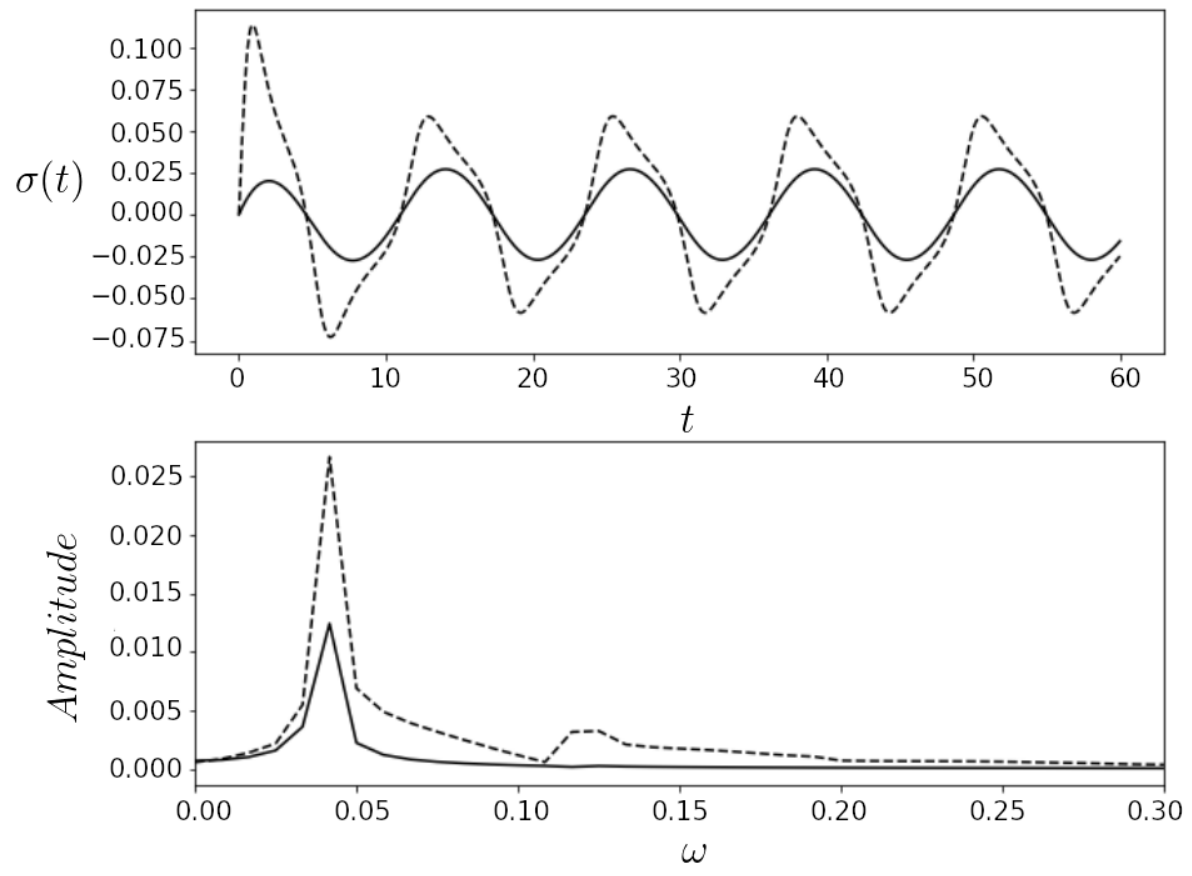

\subsubsection{Fourier Transform Framework}

The shear stress response in a LAOS test can be given as a sum of higher harmonic contributions (MELiTo et al., 2012) in Fourier decomposition

$$
\sigma^{F T}(t)=\sum_{m: o d d} I_{m}^{\prime} \sin (m \omega t)+I_{m}^{\prime \prime} \cos \left(m \omega t+\delta_{m}\right)
$$

where the Fourier coefficients $I_{m}^{\prime}$ and $I_{m}^{\prime \prime}$ are given by

$$
I_{m}^{\prime}=\frac{\omega}{\pi} \int_{-\pi / \omega}^{\pi / \omega} \sigma \sin (m \omega t) d t
$$


and

$$
I_{m}^{\prime \prime}=\frac{\omega}{\pi} \int_{-\pi / \omega}^{\pi / \omega} \sigma \cos (m \omega t) d t .
$$

These Fourier coefficients can be defined in terms of nonlinear storage and loss moduli by

$$
I_{m}^{\prime}=\gamma_{0} G_{m}^{\prime} \text { and } I_{m}^{\prime \prime}=\gamma_{0} G_{m}^{\prime \prime}
$$

where in the limit $\gamma_{0} \rightarrow 0, G_{1}^{\prime}$ and $G_{1}^{\prime \prime}$ become the usual complex moduli in the linear regime. We can also define the phase angle for each Fourier mode, $m$, as

$$
\tan \left(\delta_{m}\right)=\frac{G_{m}^{\prime \prime}}{G_{m}^{\prime}}
$$

It has been suggested that this framework is sensitive to differing levels of branching (HYUN et al.; HYUN et al.; HYUN AND WILHELM; KEMPF et al., 2007; 2006; 2009; 2013) and has the advantage that LAOS is easier experimentally than extensional flow. Typically, measured quantities include the real and imaginary odd harmonics, $I_{m}^{\prime}$ and $I_{m}^{\prime \prime}$, where $m=1,3,5, \ldots$. From these parameters, the absolute value of each harmonic is examined as a fraction of the absolute first harmonic and can be defined as

$$
I_{m / 1}=\sqrt{\frac{I_{m}^{\prime 2}+I_{m}^{\prime \prime 2}}{I_{1}^{\prime 2}+I_{1}^{\prime 2}}} .
$$

MacSporran and Spiers (MACSPORRAN AND SPIERS, 1984) have shown that LAOS is a sensitive technique for investigating the microscopic structure of fluids, in particular the phase shift for the third harmonic, $\Phi_{3}=\phi_{3}-3 \phi_{1}$, and the third storage and loss moduli, $G_{3}^{\prime}, G_{3}^{\prime \prime}$ are of special interest in characterizing a material and characterizing both viscous and elastic nonlinear rheology (NEIDHOFER et al., 2003). Wilhelm (WILHELM et al., 1998) showed that applying oscillatory shear to non-Newtonian linear polymers provides a tool for investigating nonlinear response independent 
of the material. By investigating the shear response in Fourier space, higher harmonics were used to characterize nonlinearities. That investigation continued at the cross over from linear to nonlinear behavior using the relative magnitude of the third harmonic, $I_{3 / 1}$.

Although this Fourier Transform framework is mathematically robust, it suffers from two drawbacks. First, when multiple nonlinearities are present, the Fourier spectrum can spread continuously. The amount of spectral broadening depends on the signal shape and intensity and the Fourier Transform framework lacks a clear physical interpretation of the higher-order coefficients. Second, the use of $G_{1}^{\prime}$ and $G_{1}^{\prime \prime}$ (the first harmonic coefficients) as viscoelastic moduli in the nonlinear regime is arbitrary and often fails to capture the rich nonlinearities that appear in the raw data signal.

The higher harmonic contributions are the main responsible for the nonsinusoidal shape of the shear stress waveform, with the third harmonic being the one that most affects it. Besides that, the phase angle for the third harmonic gives information about sample structure.

\subsubsection{Lissajous-Bowditch Analysis}

Ewoldt (EWOLDT et al., 2008a) characterized the complex nonlinear response in LAOS measurements based on the analysis of Lissajous-Bowditch plots, allowing the distinction between elastic and viscous nonlinearities. In a Lissajous-Bowditch plot, it is possible to illustrate the cyclic variations of shear stress as a function of strain, or shear stress versus shear rate, which occur during an oscillatory shear experiment.

In this framework the following variables were defined in order to quantify the nonlinear viscoelastic properties: the minimum strain elastic shear modulus or tangent modulus at $\gamma=0, G_{M}^{\prime}$;

and the large strain elastic shear modulus or secant modulus evaluated at the maximum imposed strain $\left(\gamma=\gamma_{1}\right)$. These variables are defined as

$$
G_{M}^{\prime}=\left.\frac{d \sigma}{d \gamma}\right|_{\gamma=0}=\frac{1}{\gamma_{1}} \sum_{m: o d d} m \sigma_{m} \cos \left(\delta_{m}\right)=\sum_{m: o d d} m G_{m}^{\prime}
$$


Figure 2.2: Dimensionless Lissajous-Bowditch for Giesekus model with parameters $\omega=0.5, \alpha=$ $0.5, \eta=0.1, \lambda^{\prime}=2$ and $\dot{\gamma}=2$. The graph on the left shows the elastic component and $G_{M}^{\prime}$ and $G_{L}^{\prime}$. The plot on the right shows the viscous stress and $\eta_{M}^{\prime}$ and $\eta_{L}^{\prime}$. [Source: the author.]
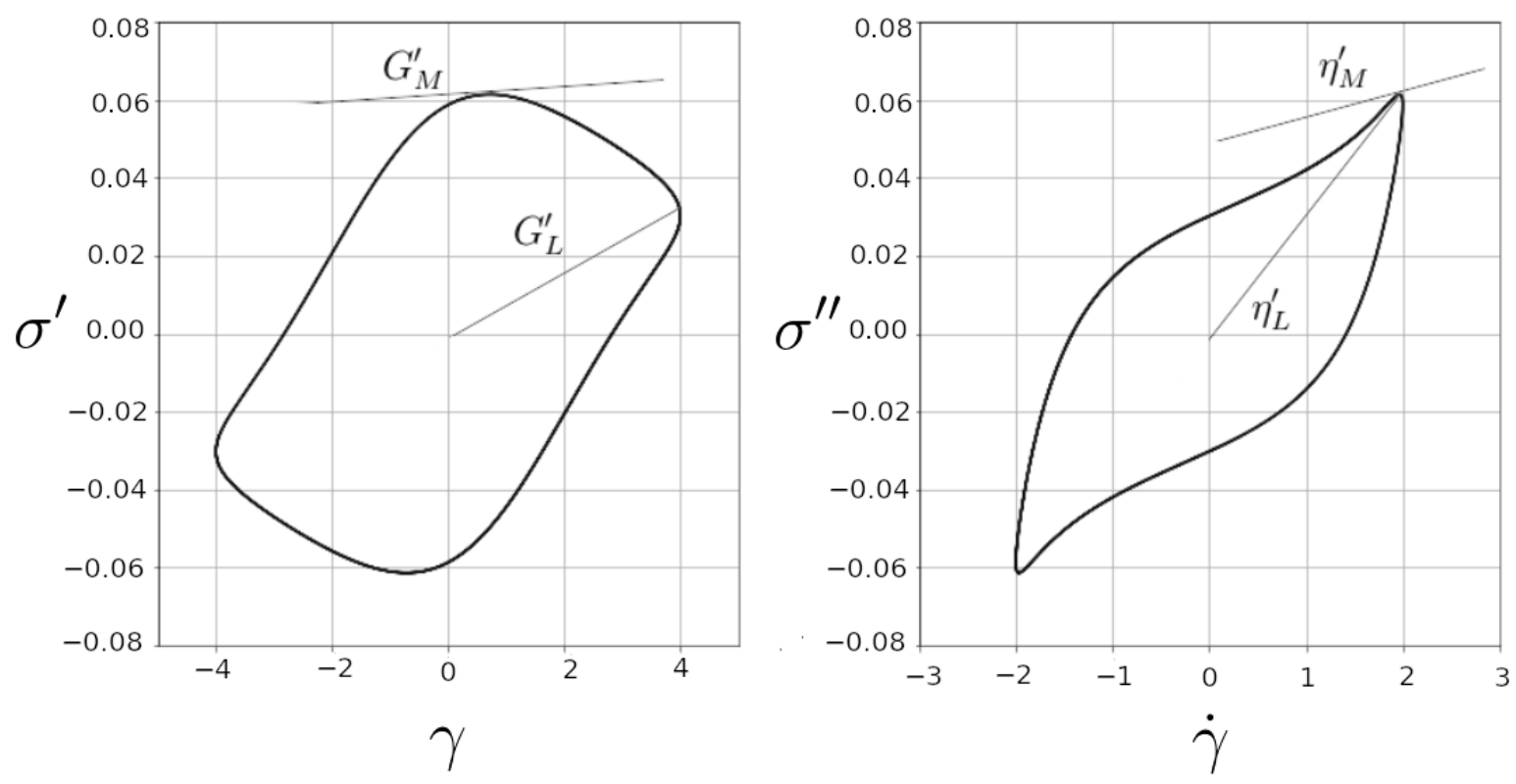

$$
G_{L}^{\prime}=\left.\frac{\sigma}{\gamma}\right|_{\gamma= \pm \gamma_{1}}=\frac{1}{\gamma_{1}} \sum_{m: o d d}(-1)^{(m-1) / 2} \sigma_{m} \cos \left(\delta_{m}\right)=\sum_{m: o d d}(-1)^{(m-1) / 2} G_{m}^{\prime}
$$

These properties can be determined graphically using the Lissajous-Bowditch plot $\sigma(\gamma)$, as shown in figures 2.2 and 2.3 or from the Fourier parameters of the higher harmonic stress contributions, by obtaining the coefficients, $G_{m}^{\prime}$, the amplitudes, $\sigma_{m}$, and the phase angles, $\delta_{m}$. Graphically, the minimum-strain modulus represents the slope of the tangent at $\gamma=0$ and the large-strain modulus represents the slope of a straight line connecting the axes origin to the point where the strain is maximum. The minimum-rate and large-rate dynamic viscosities, $\eta_{M}^{\prime}$ and $\eta_{L}^{\prime}$, respectively, can also be defined in a similar manner:

$$
\eta_{M}^{\prime}=\left.\frac{d \sigma}{d \dot{\gamma}}\right|_{\dot{\gamma}=0}=\frac{1}{\omega \gamma_{1}} \sum_{m: o d d}(-1)^{(m-1) / 2} m \sigma_{m} \sin \left(\delta_{m}\right)=\sum_{m: o d d}(-1)^{(m-1) / 2} m G_{m}^{\prime \prime}
$$


Figure 2.3: Dimensionless Lissajous-Bowditch for UPC Maxwell with parameters $\omega=0.95, \eta=1$, $\lambda^{\prime}=2$ and $\dot{\gamma}=1.5$. The graph on the left shows the elastic component and $G_{M}^{\prime}$ and $G_{L}^{\prime}$. The plot on the right shows the viscous stress and $\eta_{M}^{\prime}$ and $\eta_{L}^{\prime}$. [Source: the author.]
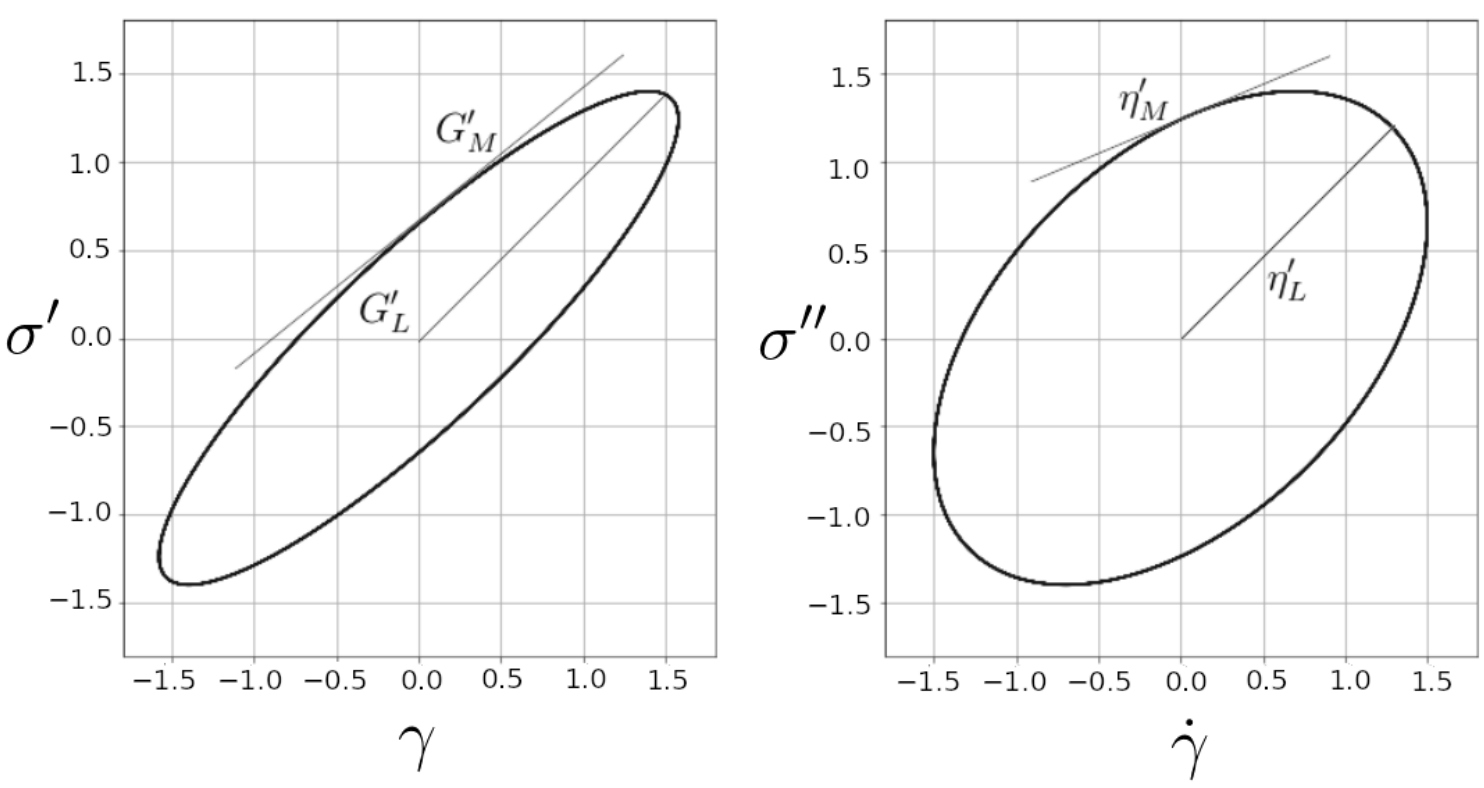

$$
\eta_{L}^{\prime}=\left.\frac{\sigma}{\dot{\gamma}}\right|_{\gamma= \pm \dot{\gamma}_{1}}=\frac{1}{\omega \gamma_{1}} \sum_{m: o d d} \sigma_{m} \sin \left(\delta_{m}\right)=\sum_{m: o d d} G_{m}^{\prime \prime}
$$

These two properties define the instantaneous viscosities at the smallest and at the largest shear rates, respectively. In a Lissajous-Bowditch plot of the form $\sigma(\dot{\gamma}), \eta_{M}^{\prime}$ represents the slope of the tangent at $\dot{\gamma}=0$ and $\eta_{L}^{\prime}$ the slope of a straight line connecting the axes origin to the point for which the shear rate is maximum.

Based on these variables, it is possible to define the strain-stiffening ratio

$$
S\left(\omega, \gamma_{1}\right)=\frac{G_{L}^{\prime}-G_{M}^{\prime}}{G_{L}^{\prime}}
$$

and the shear-thickening ratio: 


$$
T\left(\omega, \gamma_{1}\right)=\frac{\eta_{L}^{\prime}-\eta_{M}^{\prime}}{\eta_{L}^{\prime}}
$$

For $S>0$, the material shows intra-cycle strain-stiffening, whereas $S<0$ indicates intracycle strain-softening. For a linear elastic response, $S=0$. Similarly, $T=0$ represents a linear viscous response, and $T>0$ corresponds to intra-cycle shear-thickening and $T<0$ intra-cycle shear-thinning.

In Lissajous-Bowditch curves, for the case of linear viscoelastic behavior, the parametric loops of stress vs. strain are ellipses, with a minor axis that narrows with increasing frequency, i.e. less is dissipated in the high-frequency elastic region. We may see that in figure 2.4. The evolution in the shape of the distorted stress waveforms can be related with systematic changes in the internal microstructure of the material or the polymer topologies (linear or branched chain).

The third coefficients analysis presented with the Chebyshev framework can also be done based on Lissajous-Bowditch curves. The curvature (second derivative) of the elastic stress $\sigma^{\prime}(\gamma)$ with respect to input strain amplitude can indicate either strain-stiffening (positive concavity; $d^{2} \sigma^{\prime} / d^{2} \gamma>0$ ), strain-softening (negative concavity; $d^{2} \sigma^{\prime} / d^{2} \gamma<0$ ), or linear elastic behavior (zero concavity). Similarly, the curvature of the viscous stress indicates shear-thickening (positive curvature; $d^{2} \sigma^{\prime \prime} / d^{2} \gamma>0$ ) or shear-thinning (negative curvature). The magnitude of each Chebyshev coefficient typically decays monotonically with $m$, therefore to leading order positive curvature results for $e_{m}, v_{m}>0$, whereas negative curvature results for $e_{m}, v_{m}<0$.

\subsubsection{LAOS Strain Chebyshev Framework}

As stated before, it is difficult to obtain a physical understanding from the higher harmonic components of the stress response. We can represent the individual curves of the decomposed elastic, $\sigma^{\prime}$, and viscous, $\sigma^{\prime \prime}$, stresses with an orthogonal set of polynomial functions such as the Chebyshev polynomials of the first kind. This simplifies the description of the material response because it is no longer necessary to consider the explicit (and superfluous) temporal dependence in the stress, and instead focus on how the material response varies with magnitude and rate of deformation.

Usually, the rheological tests are conducted in strain-controlled environment. Cho (CHO et 
Figure 2.4: Lissajous-Bowditch for Giesekus model with parameters $\omega=0.5, \alpha=0.5, \eta=0.1$, $\lambda^{\prime}=2$. The dashed line represents the elastic stress $[\mathrm{Pa}]$ and the solid line the viscous stress $[\mathrm{Pa}]$ as a function of strain $[\mathrm{m} / \mathrm{m}]$ and strain rate $\left[\mathrm{s}^{-1}\right]$. The left plot represents the model under SAOS with $\dot{\gamma}=0.2$ and the right plot represents the model under LAOS with $\dot{\gamma}=2.2$. [Source: the author.]
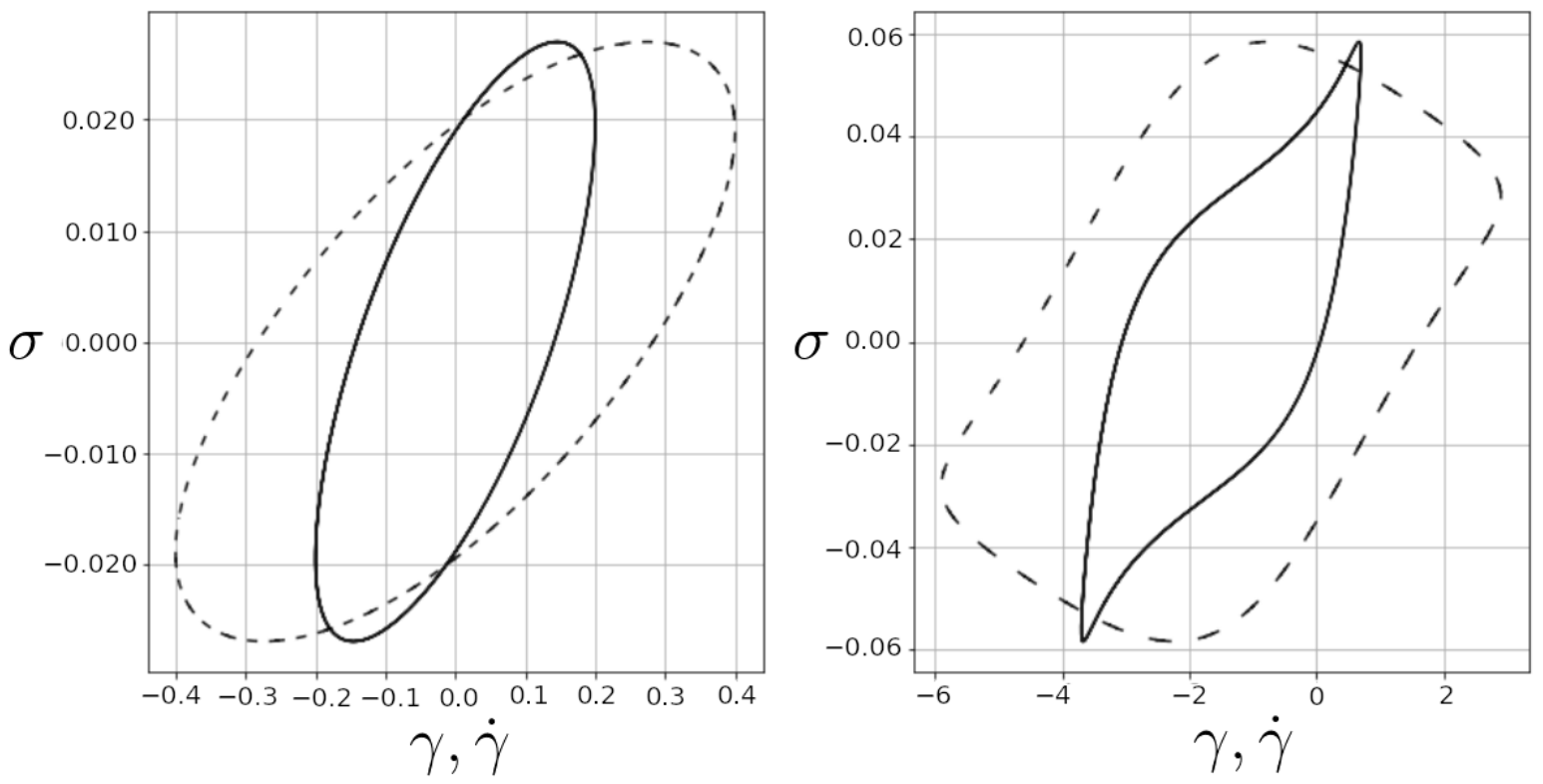

$a l ., 2005)$ used a geometrical interpretation of viscoelasticity to decompose the nonlinear response. The response to a sine-strain input is given by $\sigma(t)=\sigma^{\prime}(x)+\sigma^{\prime \prime}(y)$ and is decomposed into a superposition of elastic stress $\sigma^{\prime}(x)$ and viscous stress $\sigma^{\prime \prime}(y)$ :

$$
\begin{aligned}
& \sigma^{\prime}(\hat{x})=\frac{\sigma(\gamma, \dot{\gamma})-\sigma(-\gamma, \dot{\gamma})}{2}=\gamma_{0} \sum_{m: o d d} G_{m}^{\prime} \sin (m \omega t), \\
& \sigma^{\prime}(\hat{y})=\frac{\sigma(\gamma, \dot{\gamma})-\sigma(\gamma,-\dot{\gamma})}{2}=\gamma_{0} \sum_{m: o d d} G_{m}^{\prime \prime} \cos (m \omega t),
\end{aligned}
$$

where $\hat{x}$ and $\hat{y}$ are the normalized strain and normalized strain rate, respectively. The decomposition is based on the concept that $\sigma^{\prime}$ is odd with respect to $\hat{x}$ and even with respect to $\hat{y}$, and $\sigma^{\prime \prime}$ is even with respect to $\hat{x}$ and odd with respect to $\hat{y}$. 
The use of an orthogonal polynomial series may be contrasted with the alternative option of using a polynomial series of arbitrary order, e.g. $\sigma^{\prime}(\gamma)=a_{1}+a_{3} \gamma^{3}+a_{5} \gamma^{5}+\ldots+a_{m} \gamma^{m}$, in which the coefficients $a_{m}$ are fit by the method of least squares. Such regressions result in coefficient values $a_{m}$ which depend on the highest order of the polynomial used for the fit, in contrast to the use of orthogonality relations that enable unique determination of the Chebyshev coefficients.

A series of Chebyshev functions, with corresponding weighting coefficients, is then used to represent the elastic and viscous stresses according to

$$
\begin{aligned}
& \sigma^{\prime}(\hat{x})=\gamma_{0} \sum_{m: o d d} e_{m}\left(\omega, \gamma_{0}\right) T_{n}(\hat{x}) \\
& \sigma^{\prime \prime}(\hat{y})=\dot{\gamma}_{0} \sum_{m: o d d} v_{m}\left(\omega, \gamma_{0}\right) T_{n}(\hat{y})
\end{aligned}
$$

$T_{n}(\hat{x})$ and $T_{n}(\hat{y})$ correspond to $n$ th-order of the Chebyshev polynomials of the first kind and $v_{m}$ and $e_{m}$ are orthonormal viscous and elastic coefficients respectively. Chebyshev coefficients directly correspond one-to-one to Fourier coefficients in the time domain as explained in the previous section as follows:

$$
\begin{gathered}
e_{m}=G_{m}^{\prime}(-1)^{(m-1) / 2} \\
v_{m}=\frac{G_{m}^{\prime \prime}}{\omega}=\eta_{m}^{\prime}
\end{gathered}
$$

for $m$ : odd. Thus, similar to the third-order Fourier harmonics, the third-order Chebyshev coefficients $v_{3}$ and $e_{3}$ signal the departure from nonlinearity while also providing physical interpretation. In the linear regime $e_{3} / e_{1}<<1$ and $v_{3} / v_{1}<<1$ because the effective contribution of higher-order terms is negligible.

The third-order coefficients determine the concavity of each curve (to leading order), and 
Figure 2.5: Stress [Pa] response of the UPC Maxwell model for $\omega=0.95, \dot{\gamma}=1.5, \eta=1$, $\lambda^{\prime}=2$ as a function of time [s]. The markers represent the data itself, the dotted line is the signal reconstructed with only 1 coefficient, the dashed line shows the addition of the third coefficient and the solid line represents the signal reconstructed with five coefficients. [Source: the author.]

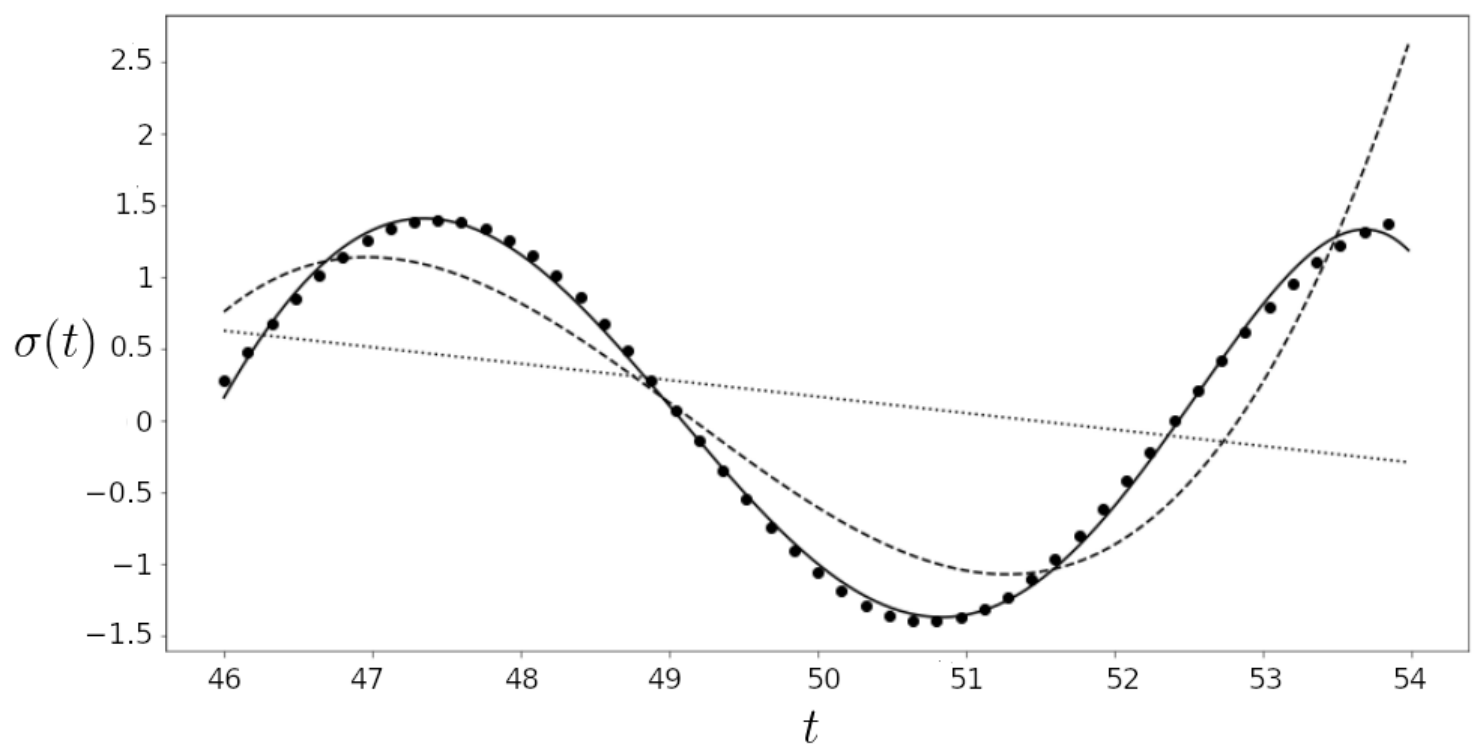

it is this curvature of the measured material response with respect to strain or strain-rate which corresponds to a physical interpretation of the nonlinearity within a steady-state oscillatory cycle (intra-cycle nonlinearities of a nonlinear waveform). For instance, in the nonlinear regime, the first-order coefficients describe the average, global or intercycle response (basis function is linear but changes for each cycle of increasing strain amplitude), whereas the third-order reveal local or intracycle responses (relative nonlinearities within a single cycle). These nonlinearities usually manifest as curvatures, characterized by upturns or bends. Figures 2.5, 2.6 and 2.7 show how the amount of coefficients change the characterization of the nonlinearities.

In Chebyshev framework, positive values of the first-harmonic nonlinearities $e_{1}>0$ and $v_{1}>$ 0 signify intercycle elastic stiffening and viscous thickening (visually observed as counterclockwise rotation or increasing slope), whereas negative values mean intercycle elastic softening and viscous thinning (clockwise rotation or decreasing slope). In the same manner, for positive contributions of the third-harmonic nonlinearities $e_{3}>0$ and $v_{3}>0$, the response is referred as to intracycle strain stiffening and intracycle shear thickening, respectively (observed as upturns). For negative contributions, the response is referred as to intracycle strain softening and intracycle shear thinning 
Figure 2.6: Dimensionless Lissajous-Bowditch curve of the elastic stress component of the UPC Maxwell model for $\omega=0.95, \dot{\gamma}=1.5, \eta=1, \lambda^{\prime}=2$. The first plot represents the data (markers) and the signal reconstructed with one coefficient. The second presents the signal reconstructed with 3 coefficients and the last with 5 coefficients. [Source: the author.]
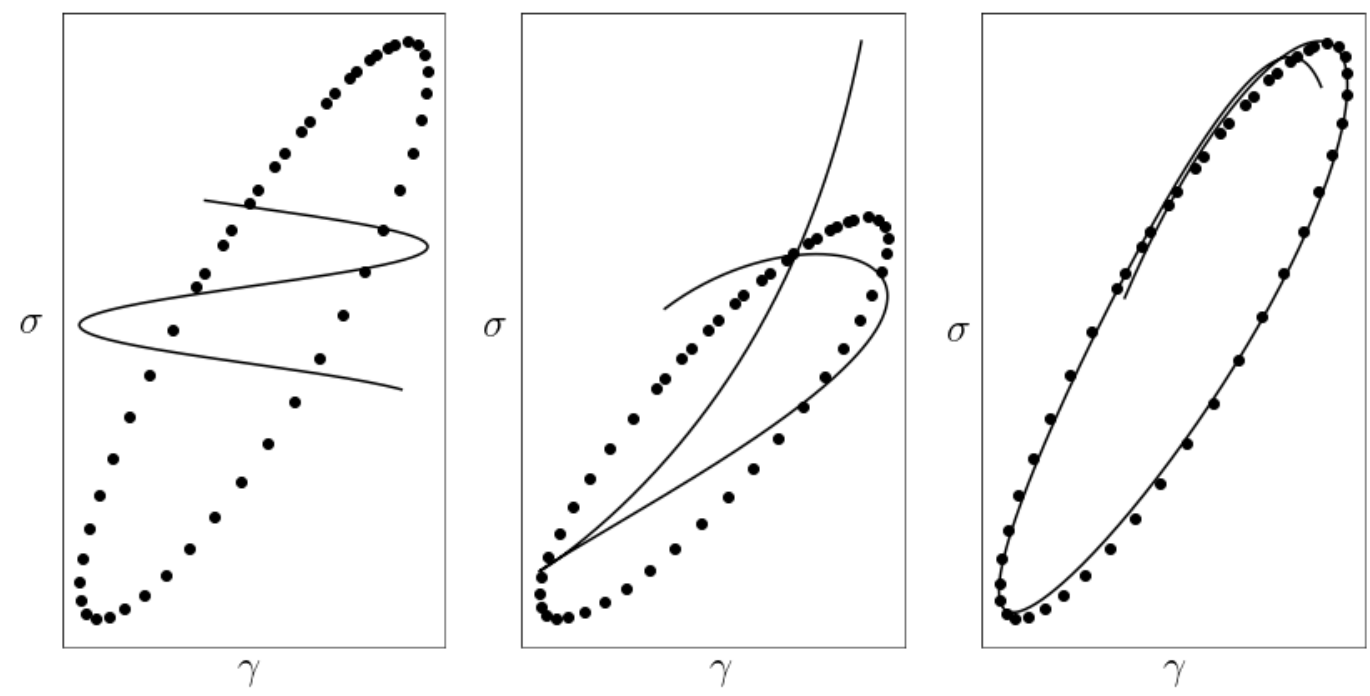

(observed as downturns).

Figure 2.8 shows the elastic $e_{n}$, and viscous, $v_{n}$, Chebyshev coefficients of Giesekus model. In this case, $S=0.6$ and $L=-0.11$, representing a strain-stiffening and shear-thinning fluid. The Chebyshev coefficients and stiffening/thickening ratios ( $S$ and $T$ ) are related in their interpretation and this analysis can also be done based on the coefficients, namely $e_{3}=G_{3}^{\prime}>1$ and $v_{3}=G_{3}^{\prime \prime} / \omega<1$. In the case of the Giesekus model, the strain stiffening is caused by the fillaments deformation. The stiff filaments deform initially by bending at small strains and then by stretching at larger strains when their end-to-end vectors align in the shear field. In this mode, fibers with linear force-extension relations can also produce strain stiffening in networks because of the geometrical changes as they align in shear. This type of fluid also presents shear thinning, a nonNewtonian behavior of fluids whose viscosity decreases under shear strain, due to the uncoil of the macromolecules. 
Figure 2.7: Dimensionless Lissajous-Bowditch curve of the viscous stress component of the UPC Maxwell model for $\omega=0.95, \dot{\gamma}=1.5, \eta=1, \lambda^{\prime}=2$. The first plot represents the data (markers) and the signal reconstructed with one coefficient. The second presents the signal reconstructed with 3 coefficients and the last with 5 coefficients. [Source: the author.]
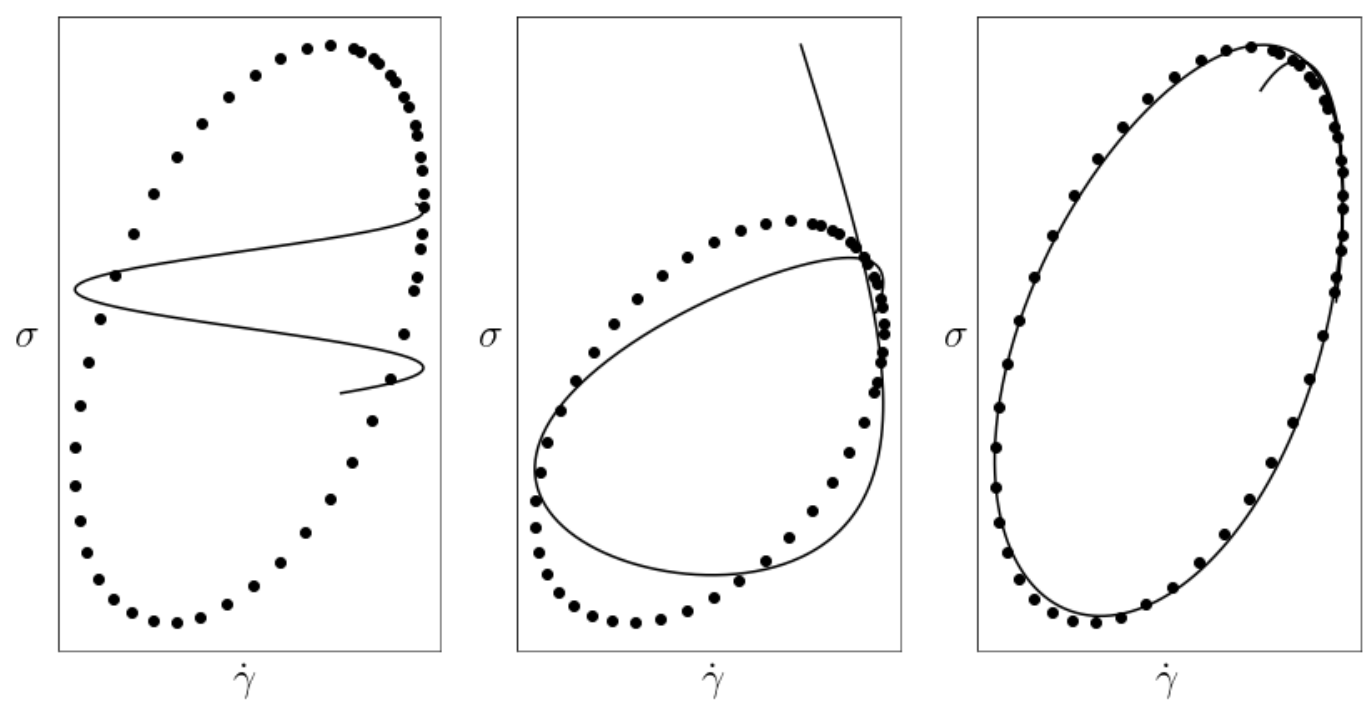

\subsubsection{LAOS Stress Chebyshev Framework}

A similar ontological framework has been proposed for stress-controlled test: an imposed cosine oscillating stress $\sigma(t)=\sigma_{0} \cos (\omega t)$ gives a strain response. The same geometry arguments can be used and the total strain can be decomposed into its elastic and viscous components defined as $\gamma(t)=\gamma^{\prime}(t)+\gamma^{\prime \prime}(t)$. However, the Chebyshev representation is given by the following equations

$$
\begin{gathered}
\gamma^{\prime}(t)=\sigma_{0} \sum_{m: o d d} J_{m}^{\prime}\left(\omega, \sigma_{0}\right) \cos (m \omega t)=\sigma_{0} \sum_{m: o d d} J_{m}^{\prime}\left(\omega, \sigma_{0}\right) T_{n}(x), \\
\gamma^{\prime}(t)=\sigma_{0} \sum_{m: o d d} m \omega J_{m}^{\prime}\left(\omega, \sigma_{0}\right) \cos (m \omega t)=\sigma_{0} \sum_{m: o d d} m \omega J_{m}^{\prime \prime}\left(\omega, \sigma_{0}\right) T_{n}(x),
\end{gathered}
$$

$J_{m}^{\prime}\left(\omega, \sigma_{0}\right)=c_{m}\left(\omega, \sigma_{0}\right)$ and $m \omega J_{m}^{\prime \prime}\left(\omega, \sigma_{0}\right)=f_{m}\left(\omega, \sigma_{0}\right)$ are the interrelations between the Cheby- 
Figure 2.8: Elastic, $e_{n}$, and viscous, $v_{n}$, Chebyshev coefficients of Giesekus model with parameters $\omega=0.1, \alpha=0.3, \eta=0.1, \lambda^{\prime}=0.5$. In this case, $e_{3}=0.0015809 P a, G_{L} / G_{M}=2.4751$, $v_{3}=-0.024187$ Pa.s, $\tan \delta_{1}=11.4853, \eta_{L}^{\prime} / \eta_{M}^{\prime}=0.89852$ and $\eta_{1}^{\prime}=G^{\prime \prime} / \omega$ obtained using MITLaos software (EWOLDT et al., 2007). [Source: the author.]
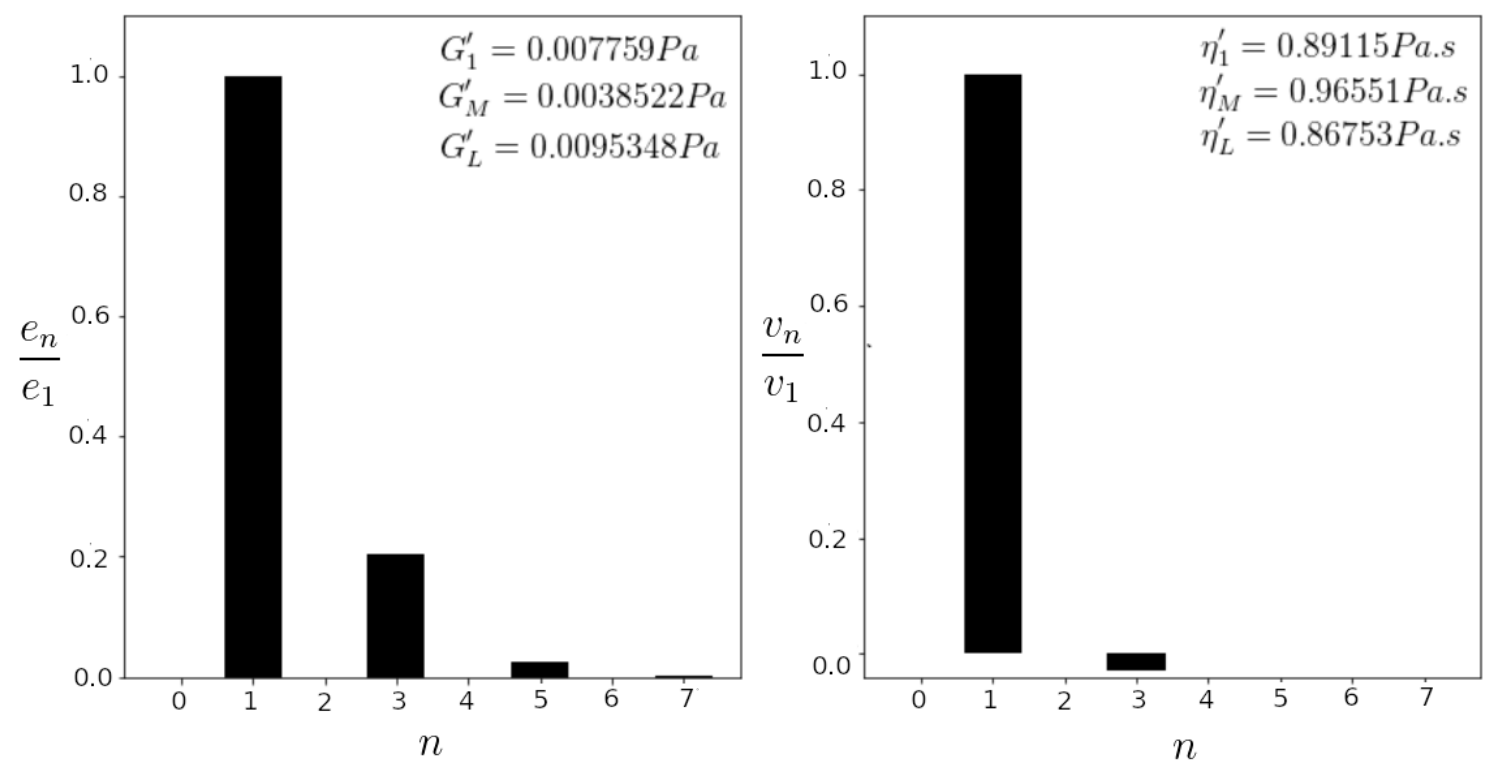

shev and Fourier coefficients. The coefficients $c_{m}$ and $f_{m}$ represent Chebyshev compliance and fluidity coefficients, respectively.

\subsubsection{Unidirectional Large Amplitude Oscillatory Shear (UD-LAOS)}

The UD-LAOS test denotes a superposition of an oscillatory shear in the nonlinear regime and a steady shear equal in amplitude to the oscillation, namely

$$
\dot{\gamma}(t)=\dot{\gamma}_{D C}+\dot{\gamma}_{0} \cos (\omega t)
$$

where $\dot{\gamma}_{D C}$ is a constant strain. UD-LAOS experiments were previously introduced to study thixotropic suspensions (ARMSTRONG et al., 2016), since the superposition of the steady shear prevents the flow from reversing. In these coordinates, the transient oscillatory stress is not zero on 
average during the cycle. Using these variables, elastic and viscous projections of the UD-LAOS stress response, i.e., Lissajous-Bowditch plots, can beconstructed in a manner similar to LAOS. In these relative coordinates, the shape of a cycle for a linearly viscoelasticfluid and a Newtonian fluid to a UD-LAOS experiment will be identical to the corresponding shape for a LAOS experi-ment. However, any nonlinear model will provide different Lissajous-Curves.

Physically, we may see the particles during the positive cycle of $\gamma_{0}$ aligning in the flow direction. However, when it enters the negative cycle, the steady shear prevents the particles from reversing their direction, mantaining its previous orientation. When dealing with nonspherical particles, UD-LAOS shows the importance of directional structure formation under shear.

This deformation can also be seen in the Lissajous-Bowditch curves in figure 2.9. These curves show the Giesekus model considering normal LAOS (dashed lines) and UD-LAOS (solid lines). We may observe that when $\dot{\gamma}_{D C} \neq 0$, there is the presence of even harmonics, that are not usual for the shear stress signal.

The presence of these even harmonics are related to the nonlinearities of the Giesekus model. When the suspension does not have any deformable or anisotropic particles, it is possible to remove the DC signal, considering it the energy at $\omega=0$. However, the nonlinear constitutive models usually promote a spreading of the energy in the frequency spectrum. Figure 2.10 shows the Lissajous-Bowditch curves for Giesekus model considering normal LAOS and UD-LAOS. 
Figure 2.9: Stress $[\mathrm{Pa}]$ as a function of time $[\mathrm{s}]$ for Giesekus model (eq. 2.25) with parameters $\omega=0.5, \dot{\gamma}=1.5, \alpha=0.5, \eta=0.1, \lambda^{\prime}=2$. The dashed line represents $\dot{\gamma}_{D C}=1.5$ and the solid line represents $\dot{\gamma}_{D C}=0$. The plot bellow shows the frequency spectrum of both signals. [Source: the author.]
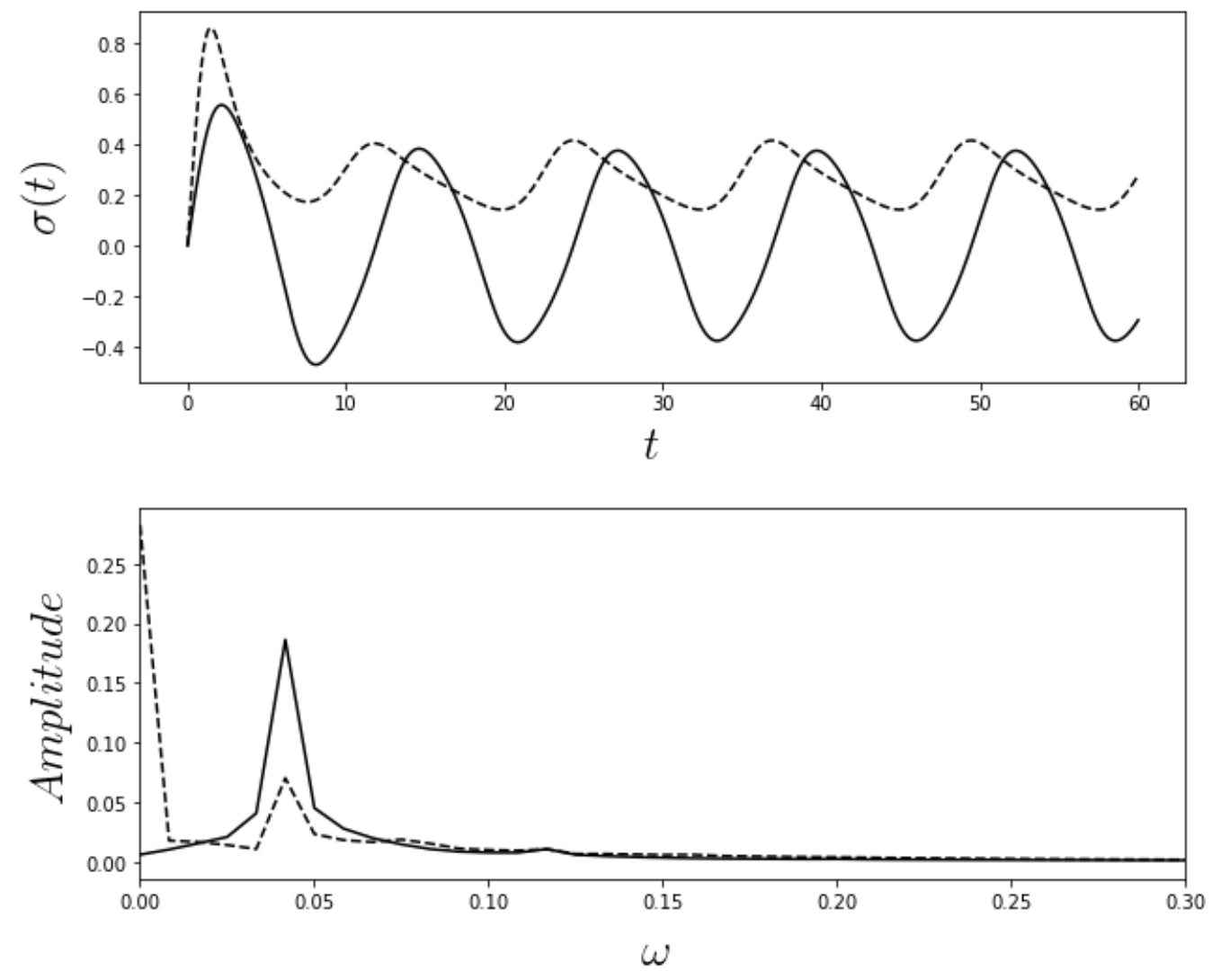
Figure 2.10: Lissajous-Bowditch curves of Giesekus model with parameters $\omega=0.5, \dot{\gamma}=1.5$, $\alpha=0.5, \eta=0.1$ and $\lambda^{\prime}=2$. The graph on the left represents elastic stress $\sigma^{\prime}$ (dashed line) and the viscous stress $\sigma^{\prime \prime}$ (solid line) $[\mathrm{Pa}]$ as a function of strain $[\mathrm{m} / \mathrm{m}]$ and strain rate $\left[\mathrm{s}^{-1}\right]$ for $\dot{\gamma}_{D C}=0$. The plot on the right represents elastic stress $\sigma^{\prime}$ (dashed line) and the viscous stress $\sigma^{\prime \prime}$ (solid line) for $\dot{\gamma}_{D C}=1.5$. [Source: the author.]
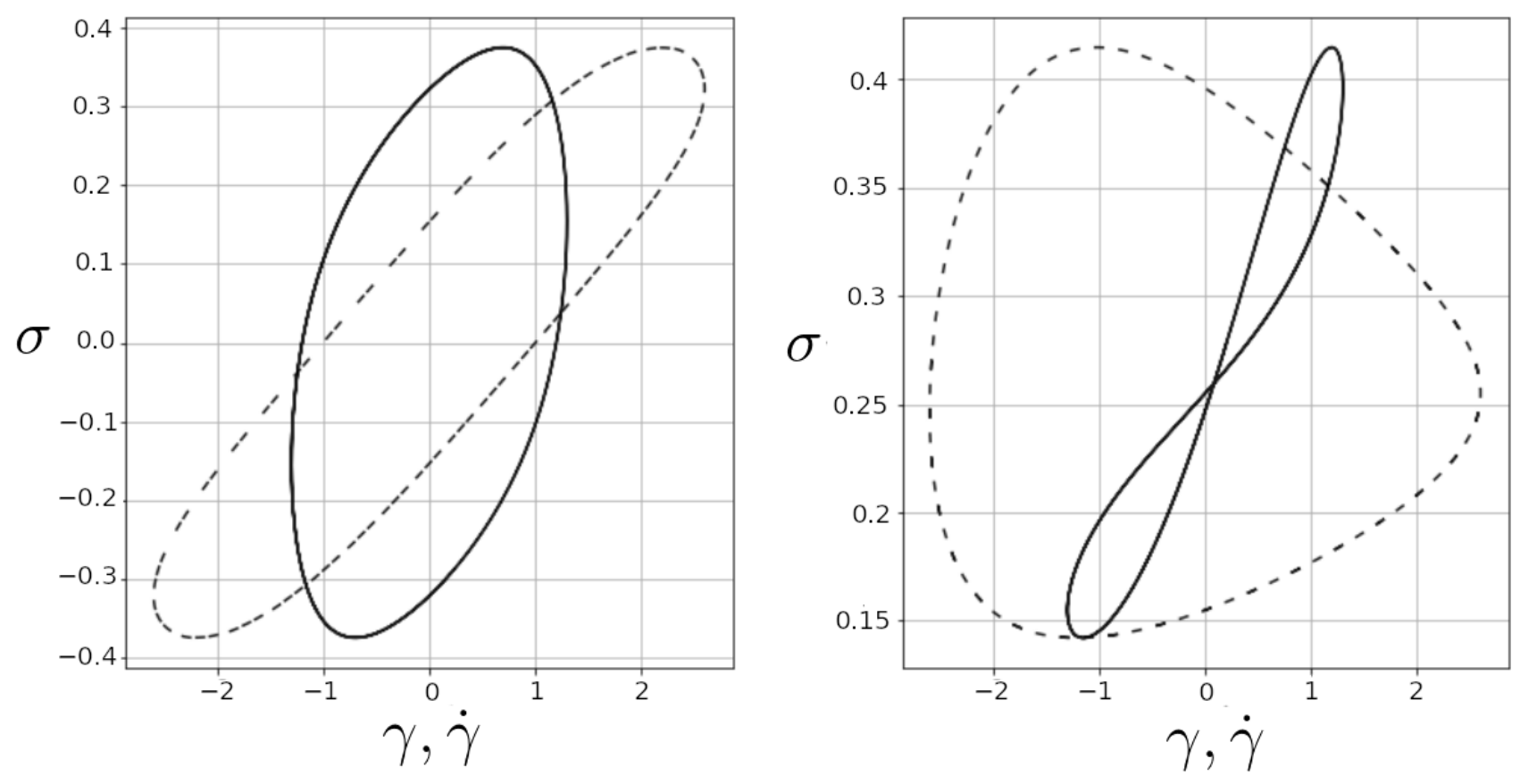


\section{Kinematic Analysis}

The present chapter aims to show a statistical analysis on $C$. elegans size, wavelength and velocity distribution when a population of nematodes crawls on agar gel. One of the most relevant features of this work is to highlight the difference, in terms of locomotion, of well-fed and starving nematodes. This kinematic analysis was made using dynamic system tools.

Although several works have been done in the past exploring the propulsion of microorganisms immersed in liquids, specific informations regarding C. elegans locomotion in high viscosity media (such as gels) with detailed information on the physical quantities, from the perspective of a kinematic study is still a poorly explored area. This is an important field that could apply classical physical theories (GRAY AND LISSMANN, 1964) regarding propulsion and locomotion in low-Reynolds number to provide a deeper understanding of some fundamental questions of active matter. There are still several open questions in this field, mostly related to the characterization of this kind of material. Since the particles have now a metabolism and hence there is an input of energy within the fluid in which they are immersed, the properties of this complex material may not be defined in a state of thermodynamic equilibrium (FODOR AND ET AL; TAKATORI AND BRADY, 2016; 2014).

It is very important to understand how the nematodes move in order to compute their bulk behavior in rheological properties. This chapter is a starting point regarding the understanding of how the metabolic conditions change the collective behavior of a population of worms. The relative importance between the forces and time scales involved in the dynamics of the living particles (or nematodes) is also discussed and physically interpreted. Finally, we provide a spectral analysis of the motion of several individuals and show how secondary frequencies are able to break the time-reversibility to which these worms are subjected and produce a highly efficient motion in low Reynolds number flow.

Experiments in a gel-like medium were performed using the nematode $C$. elegans. The high viscosity gel considered in this work is a mixture of water and agar molecules. During the nematode's crawling motion, fluid is released from the medium in a process called syneresis, which consists of the breaking of the agarose molecules bonds. The nematode then crawls in a thin water film that is formed around its body on a lubrication regime. The crawling behaviour of the nematode C. elegans (N2 wild type) immersed in a gel (NGM-agar plates with Escherichia coli) was investi- 
gated in a sealed acrylic chamber that was $2 \mathrm{~cm}$ in diameter and $1 \mathrm{~mm}$ in depth using a microscope and a high-speed camera.

\subsection{Experimental methods}

\subsubsection{Preparation of growth media}

The protocol of Stiernagle (STIERNAGLE, 2006) to produce the gel-like nematode growth medium (NGM) consists on producing a mix of $3 \mathrm{~g}$ of $\mathrm{NaCl}, 17 \mathrm{~g}$ of agar and $2.5 \mathrm{~g}$ of peptone in a 2 liter Erlenmeyer flask. After that, $975 \mathrm{ml}$ of $\mathrm{H}_{2} \mathrm{O}$ was added and the flask was autoclaved for 50 minutes with its mouth covered with aluminium foil. The solution was cooled in $55^{\circ} \mathrm{C}$ water bath for 15 minutes. Using sterile procedures, $1 \mathrm{ml}$ of $1 \mathrm{~mol} \mathrm{CaCl}_{2}$ and $1 \mathrm{ml}$ of $5 \mathrm{mg} / \mathrm{ml}$ cholesterol were added in ethanol. Next, the solution was swireled with $1 \mathrm{ml}$ of $1 \mathrm{~mol} \mathrm{MgSO}_{4}$ and $25 \mathrm{ml} 1 \mathrm{~mol}$ $\mathrm{KPO}_{4}$. This NGM solution was dispensed into petri plates using a peristaltic pump and the plates were filled with $2 / 3$ agar. The plates were left at room temperature for 3 days to allow the detection of contaminants and the moisture to evaporate.

Approximately $0.5 \mathrm{~mL}$ of $E$. coli OP50 liquid culture was applied onto the plates. The lawns grew overnight at room temperature for 8 hours. To produce the starving sample, the nematodes were transfered to other plates using two different techniques. A quick and convienient method is to move a chunk of agar from an old plate to a fresh one. Due to the fact that hundreds of nematodes are present in the chunks, this is a good technique for old plates, since the worms will probably be burrowed into the agar. An alternative approach is to use a worm picker. A 32 gauge platinum wire was attached to the tip of a Pasture pipet. The wire was flamed between transfers to avoid contamination. The end of the wire was also flattened and bent, forming a hook. A blob of bacteria was added inside the hole of the hook and it was gently swiped at the side of the worm and lifted up. The worm stuck to the bacteria and after the worm picker is slowly lowered to the new plate, it crawls off the picker. 


\subsubsection{Experimental protocol}

The main part of the experiment was the nematode tracking, which is used for obtaining kinematic data such as swimming speed, beating frequency, and amplitude. The individuals were observed through an Olympus UC30 CCD camera coupled to an Olympus BX51 microscope. Images were recorded with the focal plane at the centre of the chamber to avoid movies with nematode-wall interactions; out-of-plane recordings were discarded. The nematodes swimming kinematics were obtained from the videos using the commercial software WormLab (ROUSSLET et al., 2014). The software extracts the nematode's centroid, head and tail positions based on body shape and computes kinematic quantities. The nematode crawling produces the extraction of fluid from the gel. This fluid is mostly composed of a water-like buffer solution (M9 salt solution, $\mu=1 \mathrm{mPas}$ ). Our initial experiments showed that $C$. elegans exhibits a predominately two-dimensional sinusoidal beating pattern, producing a travelling wave that moves from head to tail. The head, tail, and centroid trajectories were analyzed using an in-house Fast Fourier Transform algorithm. Figures 3.1 and 3.2 show how the software WormLab identify the nematodes and obtain kinematic data.

Figure 3.1: Bending angle at the midpoint. [Source: Rousslet et al. (2014).]

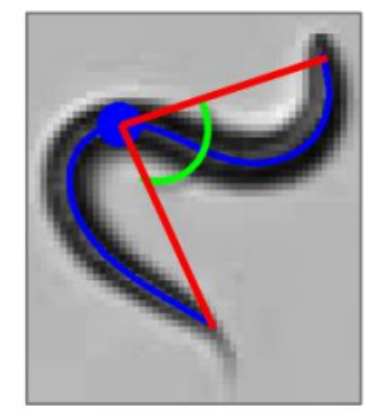

Figure 3.2: Period of the sine wave the best fits the worm's posture. [Source: Rousslet et al. (2014).]

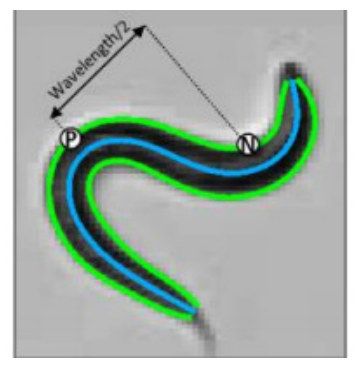

Kinematics data consisted of an average of 20 individuals analyzed in 20 different recordings. The statistical distribution of the samples can be seen in figures 3.3 and 3.4. These figures illustrate 
the size distribution, in terms of length, of the samples of well-fed and starving nematodes. It can be seen that in average the nematodes size distribution of our well-fed and starving samples did not differ substantially. Thus, this geometrical difference is not the determinant factor that dictates their differences in terms of motion (dynamic behavior). Therefore, the metabolic feature can be isolated in terms of how it affects the worm's kinematics.

Figure 3.3: Cumulative distribution function (CDF) of the standard normal distribution and frequency histogram of well-fed sample. In this case, $85 \%$ of individuals were smaller than $500 \mu \mathrm{m}$ and the length of most individuals varied between $300 \mu \mathrm{m}$ and $400 \mu \mathrm{m}$. [Source: the author.]
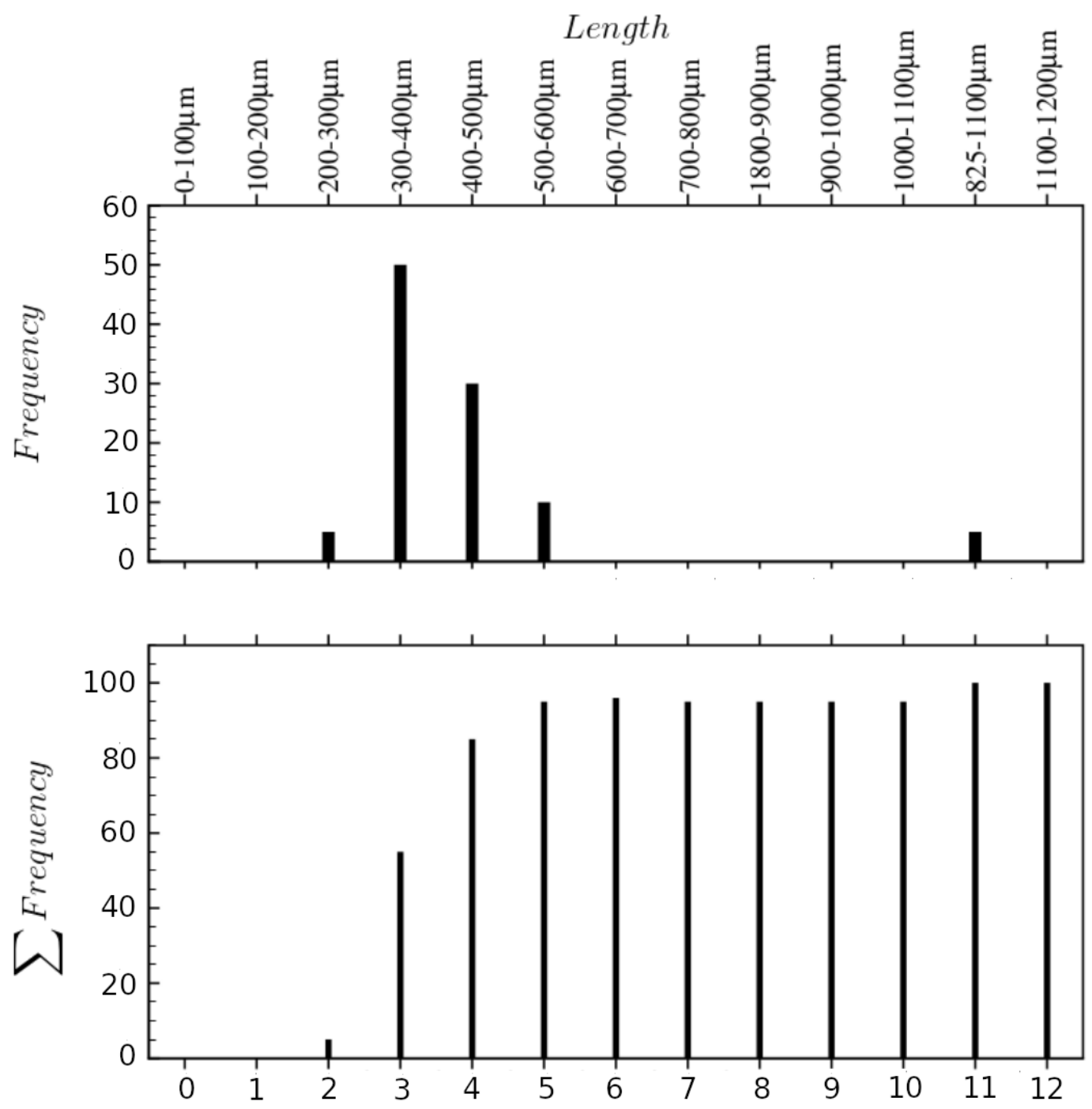
Figure 3.4: Cumulative distribution function (CDF) of the standard normal distribution and frequency histogram of the starving sample. The CDF shows that $70 \%$ of individuals were smaller than $600 \mu \mathrm{m}$ and the length of most nematodes varied between $300 \mu \mathrm{m}$ and $400 \mu \mathrm{m}$. [Source: the author.]
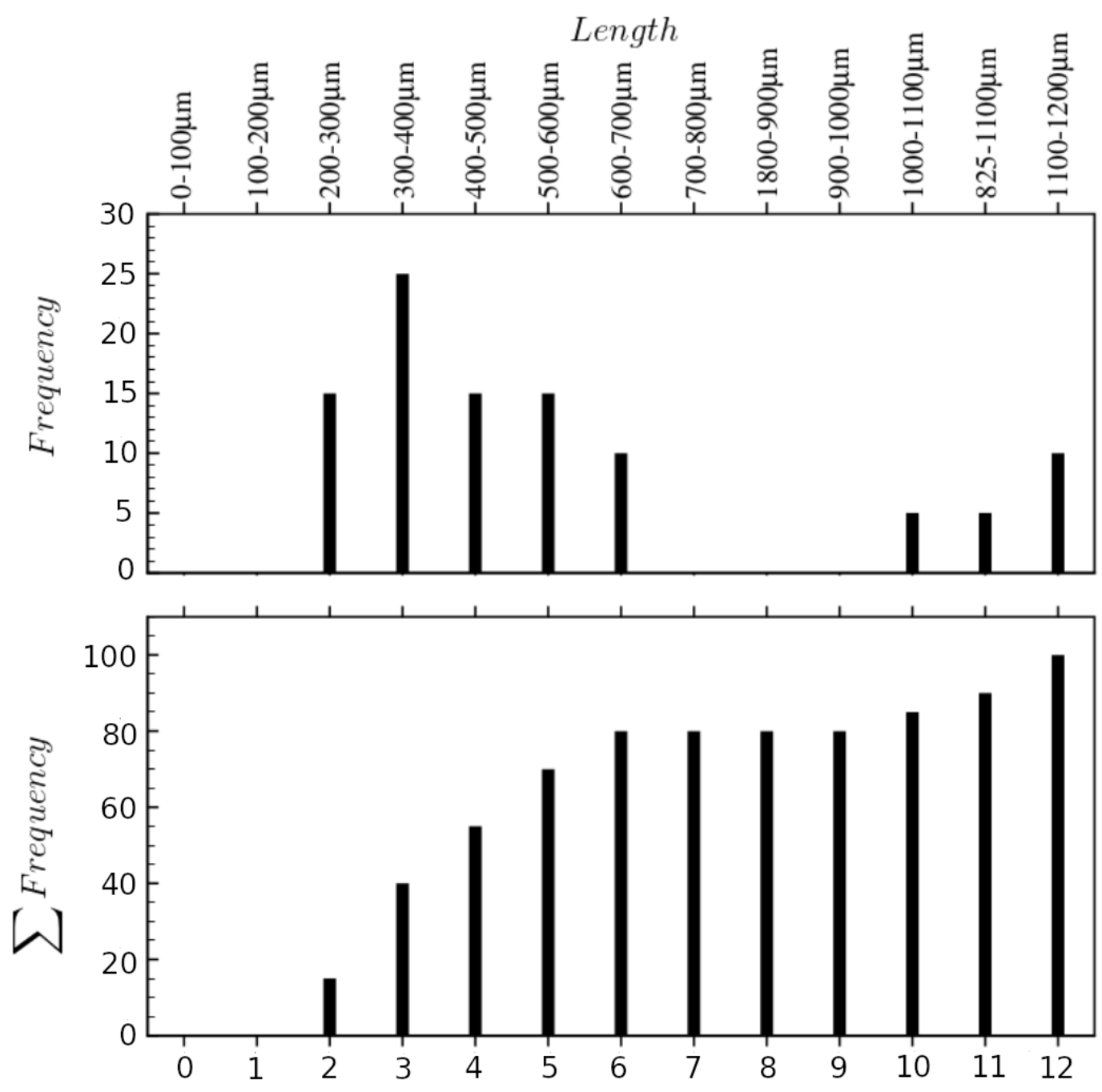


\subsection{Results and discussions}

\subsubsection{Preliminary characterization}

The typical crawling motion of $C$. elegans was studied considering the geometry and variables defined in figure 3.5, where $a$ is the worm diameter, $\lambda$ represents the nematode's motion wavelenght, $\delta$ is the $C$. elegans motion amplitude, $\theta$ represents the bending angle and $v$ the centroid velocity. The nematode's length is given by $L$.

A statistical analysis based on two populations of 20 individuals each was performed. In the first population the nematodes were immersed in a gel medium with food (E. coli bacterial suspension). For the second population of individuals, food was not available. The populations were evaluated in different days (during one week) and in different growing stages. The idea of this preliminary analysis was to check whether different environmental conditions from a biological perspective could affect the nematode's motion in two very similar surrouding media.

Figure 3.5: Geometrical sketch of variables $a, \delta, \lambda, \theta$ and v. [Source: the author.]

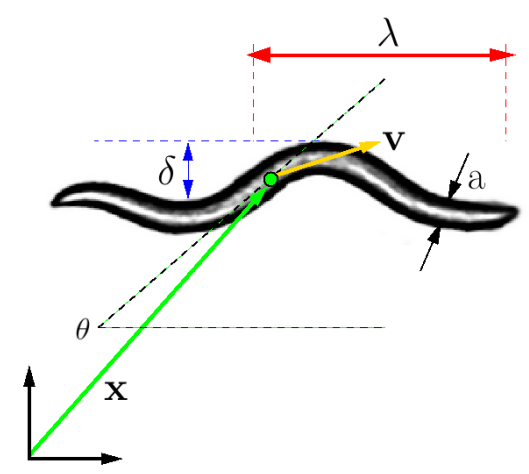

The kinematic characteristics of the individuals were analyzed based on statistics over the population of 40 individuals (including the well-fed and the starving samples) in 40 different experiments. The relevant results of this analysis are presented in tables 3.1 and 3.2. The slip parameter, $\alpha$, was defined as $\alpha=1-U_{c} / U_{v}$ where $U_{c}$ is the nematode speed with respect to a fixed frame of reference and $U_{v}=\lambda f$ is the wave speed with $f$ being the main frequency of the nematode's motion. Usually when the trace left by the nematode in the base fluid is similar to an harmonic wave motion, $\alpha$ tends to zero. That means that nearly all bending force was converted into propulsion. 
Table 3.1: Nematodes motion characterization in the absence of food. The variables $\delta, L$ and $a$ are independent of the presence or absence of food. [Source: the author.]

\begin{tabular}{cccc} 
Kinematic variable & Range & Average & Standard Deviation \\
\hline$\delta(\mu \mathrm{m} \mathrm{m})$ & $7.48-38.33$ & 19.79 & 10.65 \\
$L(\mu \mathrm{m})$ & $193.14-1160.45$ & 515.55 & 348.89 \\
$\lambda(\mu \mathrm{m})$ & $101.92-613.07$ & 276.87 & 183.95 \\
$v\left(\mu \mathrm{m} \mathrm{s}^{-1}\right)$ & $25.51-170.5$ & 76.89 & 42.10 \\
$a(\mu \mathrm{m})$ & $26.36-74.49$ & 43.20 & 17.31
\end{tabular}

Table 3.2: Well-fed nematodes motion characterization. The variables $\delta, L$ and $a$ are independent of the presence or absence of food. [Source: the author.]

\begin{tabular}{cccc} 
Kinematic variable & Range & Average & Standard Deviation \\
\hline$\delta(\mu \mathrm{m})$ & $5.7-158.2$ & 22.02 & 33.82 \\
$L(\mu \mathrm{m})$ & $185.49-1073.24$ & 336.11 & 189.36 \\
$\lambda(\mu \mathrm{m})$ & $98.14-640.38$ & 179.18 & 115.20 \\
$v\left(\mu \mathrm{m} \mathrm{s}^{-1}\right)$ & $24.56-88.94$ & 47.79 & 18.02 \\
$a(\mu \mathrm{m})$ & $17.29-68.71$ & 33.742 & 11.16
\end{tabular}

\subsubsection{Scalings arguments}

Now, we shall provide a brief description of our living system. Let's consider a typical travelling wave $F=\sin ((k x-\omega t)$ propagating from left to right with velocity $c=\omega / k$. When the nematode exerts a force on the agar plate it causes syneresis, or the extraction of water from a gel. The quantity of released water is inversely proportional to the agarose concentration squared (DumitriU, 1998). In order to move, the nematodes must bend so that the yield stress force $F_{y} \sim \tau_{0} a^{2} \alpha$ of the surrounding medium is exceeded. Here $\tau_{0}$ is the yield stress of the agar gel. This bending is directly related to the biological characteristics of the nematodes. The nematode body wall is composed of a cuticle and a single layer of longitudinal muscle cells. In nematodes, the cylindrical shape of the body tube is maintained when punctured and its diameter changes only slightly. However, nematodes are unique among worm-like organisms in lacking circumferential muscles; therefore their motion is limited to what can be accomplished by applying longitudinal forces. In the case of bending, the net force due to increased muscle tension would shorten only one side of the local body tube, while the other side would strech by the equilibrium of forces there.

A typical scale for this bending force is $F_{b} \sim M_{f} / \lambda$, where $M_{f}$ represents the nematode bending moment. We may write this bending moment as a function of the elastic modulus $E$, the 
moment of inertia and the curvature of the nematode, scaling it with $M_{f} \sim E a^{4} \delta / \lambda^{2}$. The viscous force, on the other hand, scales with the slip coefficient as $F_{\mu} \sim \mu \alpha f \lambda^{2}$, with $\mu$ being the carrier liquid viscosity.

The following calculation is based on the constitutive relation for the moment $M(s, t)$ in an inextensible filament of size $s$, representing the $C$. elegans. The total moment may be considered as $M=M_{p}+M_{a}$, where $M_{p}(s, t)$ is the passive moment and $M_{a}(s, t)$ is the active moment generated by the muscles of the nematode. The passive moment is given by the viscoelastic Voig model (KOELLER, 1950). This constitutive relation is given by:

$$
M_{p}=E I k+\mu I \frac{\partial k}{\partial t}
$$

where $k(s, t)$ is the curvature along the nematode and $I$ is the nematode moment of inertia, considered to be a hollow cylindrical shell (SZNITMAN et al., 2010).

Another force involved in the motion of the nematode is the inertial force on the liquid (which we will show to be negligible in this problem), $F_{i} \sim \rho \alpha f v \lambda^{2} L$, where $\rho$ is the fluid density. Based on statistics over 40 individuals, including starving and well-fed nematodes, the average slip for 40 analyzed individuals was 0.009. In this work, we have considered the elastic Young modulus proposed by Arratia et al. (SZnitman et al., 2010). The average Young modulus used was 2659.7Pa and the calculated average Reynolds number was 0.035 . Here, the Reynolds number is defined in the standard form as being $R e=\rho v L / \mu$, where $\mu$ is the fluid viscosity, $v$ is the nematode's velocity and $L$ its length. The bending number is defined as $B e=F_{b} / F_{\mu}$. The agarose yield stress is also important for the scaling analysis. When the nematode's bending tension is larger than the yield stress tension, the agarose gel behaves as a Newtonian fluid. In this condition, we may introduce the Bingham number as being $B h=F_{y} / F_{\mu}$. We may notice that $F_{b} / F_{y} \sim 1$. Table 3.3 shows the main dimensional quantities, forces and nondimensional physical parameters of our living system. This scaling analysis represents every possible movement of the nematode.

In this work, most of the analysis was done on worms crawling with no slip. However, slip can be extremely important in several instances. Gray and Lissman (GRAY AND LISSMANN, 1964) concluded that $\frac{U_{c}}{U_{v}}=0.9$ was a usual value for nematodes crawling on agar jelly. On the other hand, this relation is much smaller when the nematodes are crawling rigid surfaces such as moist glass or swimming. Indeed, swimming is related to a C-shape movement and it is simply an extreme phase of an S-shaped travelling wave with wavelenght longer than the worm's body (BERRI et al., 2009). 
Table 3.3: Characterization of the main dimensional quantities, forces and physical parameters averages of the nematodes locomotion. [Source: the author.]

\begin{tabular}{|c|c|c|}
\hline Dimensional & Forces & Nondimensional \\
\hline$a \approx 3.80 \times 10^{-5} \mathrm{~m}$ & & \\
\hline$\delta \approx 2.10 \times 10^{-5} \mathrm{~m}$ & & \\
\hline$\lambda \approx 2.28 \times 10^{-4} \mathrm{~m}$ & & \\
\hline$L \approx 4.25 \times 10^{-4} \mathrm{~m}$ & $F_{\mu} \sim \mu \alpha f \lambda^{2} \approx 1.4 \times 10^{-13} \mathrm{~N}$ & $\alpha \sim 10^{-3}$ \\
\hline$f \approx 3.00 \times 10^{-1} s^{-1}$ & $F_{b} \sim M_{f} / \lambda \approx 8.8 \times 10^{-9} \mathrm{~N}$ & $F_{b} / F_{\mu}=B e \sim 10^{4}$ \\
\hline$v \approx 6.20 \times 10^{-5} \mathrm{~ms}^{-1}$ & & \\
\hline$E \approx 2.60 \times 10^{3} \mathrm{~Pa}$ & $F_{i} \sim \rho \alpha f v \lambda^{2} L \approx 3.7 \times 10^{-15} \mathrm{~N}$ & $F_{i} / F_{\mu}=R e \sim 10^{-2}$ \\
\hline $\begin{array}{c}\tau_{0} \approx 5.00 \mathrm{~Pa} \\
\mu \approx 1.00 \times 10^{-3} \mathrm{Pas}\end{array}$ & $F_{y} \sim \tau_{0} a^{2} \approx 7.22 \times 10^{-9} \mathrm{~N}$ & $F_{y} / F_{\mu}=B h \sim 10^{4}$ \\
\hline$M_{f} \approx 2.00 \times 10^{-12} \mathrm{Nm}$ & & \\
\hline$\rho \approx 1.00 \times 10^{3} \mathrm{~kg} \mathrm{~m}^{-3}$ & & \\
\hline
\end{tabular}

In our condition, the travelling wave is considered to be stationary relative to the substrate, thus the slip tends to zero. Considering Parida work (PARIDA AND PADMANABHAN; PARIDA $e t$ al., 2016; 2017), it is possible to still use the same scaling analysis. For example, acoording to the same mentioned work, when the elastic modulus of the medium increases, the amplitude of the worms motion monotonically decreases. Our scale analysis estimates that the bending force $F b=E a^{4} \delta / \lambda^{3}$ is proportional to the worms amplitude $\delta$. Thus, a stiffer medium demands a lower bending force for the nematode to move. However, the medium stiffness is inversely proportional to the lubrication film thickness. In this condition, the lateral slip increases and the forward locomotion becomes less efficient. On the other hand, as mentioned by Parida (PARIDA AND PADMANABHAN; PARIDA et al., 2016; 2017), the stiffness of the medium may induce other characteristic shapes. Besides, during the animals reversal, for example, there is no sinusoidal pattern. As a matter of fact, a sharp turn (omega turn) results in approximately $180^{\circ}$ change in locomotion. During the turning process, the animal supresses its lateral head movements and there is a deep ventral head bend (AKEMA et al., 2005). In all these cases, the scaling analysis presented on table 3.3 represents the correct forces coupling considering the nematode and the agar dimensional quantities.

We postulate that, locally, viscous effects may be extremely relevant. In the sharp edges of the nematode's tail and head, there is a concentration of viscous stresses. Although globaly, in the nematode's wavelenght scale, viscous and inertial effects are dominated by bending forces, there is a concentration of viscous stresses in a minor scale, related to the edges of the nematode's body. In this scale, the nematode is forced to move its parts in a highly non-linear and non-harmonic way in several higher frequencies. This motion emulates a flagelum-like motion and breaks the kinematic 
reversibility in which its solid boundaries are bonded.

This type of behavior is also explained anatomically. Head and body movements are controlled independently by distinct classes of motor neurons and muscles (PIRRI AND AlKemA, 2012). While the body bends are restricted to the dorsal-ventral plane, the animal can flex its head in three dimensions on specific conditions; this is called nictation. It is interesting to highlight that even though this is not common, nictation may be observed on old plates with contaminating fungi. In this scenario the hyphal tips serve as the projections necessary for adherence and nictation (LEE et al., 2011). However, on standard agar plates, C. elegans movement is limited to two dimensions, preventing the observation of a three-dimensional activity such as nictation, unless the nematode is crawling inside the substrate. Head muscles are divided into eight radial symmetric sectors, and these are independently innervated by ten classes of motor neurons (WARE et al.; WHITE et al., 1975; 1986). As a consequence of this motor circuitry, worms can move their head through $360^{\circ} \mathrm{C}$.

From table 3.3 we may also conclude that the living suspension may be treated in the creeping flow regime, since the ratio $F_{i} / F_{\mu}=R e \ll 1$. It is also important to notice that the highest force involved in the problem is the nematode's bending force. This force is in the same order of magnitude as the yield stress forces and hence the bending of the nematode's muscles is capable of breaking the agar molecules of the gel, releasing water (syneresis effect). It is also important to highlight that the bending forces are much higher than the viscous forces $F_{b} \gg F_{\mu}$. It is worth to emphasize that we do not consider elastic forces on the surrounding medium in this work. The syneresis effect is responsible for the nematode to crawl in a viscous fluid which is very similar to water, different from that used in the work of Keim et al. (KEIM et al., 2012).

\subsubsection{Theoretical prediction - Length and wavelength relation}

Due to their small size, microorganisms such as bacteria, sperm cells, nematodes and various kinds of protozoa move in the low Re number regime. In such a regime, linear viscous forces dominate nonlinear inertial forces (BRENNEN AND WINET; CHILDRESS; VogEL, 1977; 1981; 1994) and locomotion must result from non-reciprocal motion in order to break time-reversal symmetry. This is the so-called "scallop theorem". Taylor (TAYLOR, 1951) demonstrated that a slender body could swim at low Reynolds number by generating traveling waves along its body. Nematodes, such 
as C. elegans, produce bending waves in order to move, which is an example of non-reciprocal motion. These bending waves consist of alternating phases of dorsal and ventral muscle contractions driven by the neuromuscular activity (WHITE et al., 1986).

Lauga (LAUGA AND Powers, 2009) postulated that the scallop theorem does not hold for groups of more than one body. In this sense, a body undergoing reciprocal motion cannot swim, however two bodies undergoing reciprocal motion with nontrivial phase differences are able to take advantage of the unsteady nature of the generated flow to move. These two bodies are able to create a collective dynamics. This collective behavior is present in solutions with $C$. elegans. However, all analyses in this chapter were made considering isolated individuals, disregarding hydrodynamic interactions.

Several observations by optical microscopy and video camera indicated that the nematode motion was harmonic, so the shape of an individual worm could be expressed, during its motion, by the following curve

$$
g(x)=C \sin \left(\frac{2 \pi x}{\lambda}\right),
$$

where $g(x)$ represents a smooth repetitive oscillation and $x$ is the position on the horizontal direction on which the wave propagate. The wavelenght $\lambda$ describes the distance the wave propagates between two valleys of the sinusoidal curve and $C$ is a calibration parameter. The total length of this curve is obtained through

$$
L(\lambda)=\int_{0}^{\lambda}\left[1+\left(\frac{d g}{d x}\right)^{2}\right]^{1 / 2} d x
$$

with $C=\lambda / 3$ calibrated by experimental data. Now, replacing eq. (3.2) in (3.3) and performing the integration, we have

$$
L(\lambda)=\left[\frac{2 \sqrt{9+4 \pi^{2}} E\left(\frac{4 \pi^{2}}{9+4 \pi^{2}}\right)}{3 \pi}\right] \lambda,
$$

where $E(x)$ represents the elliptical integral of first kind so the expression can be simplified to

$$
L(\lambda) \approx 1.72677 \lambda \text {. }
$$


Figure 3.6: The average nematode size based on statistics over 15 starving individuals (black circles) and 15 well-fed nematodes (non-filled circles) as a function of the wavelength compared to the theoretical prediction (continuous line). [Source: the author.]

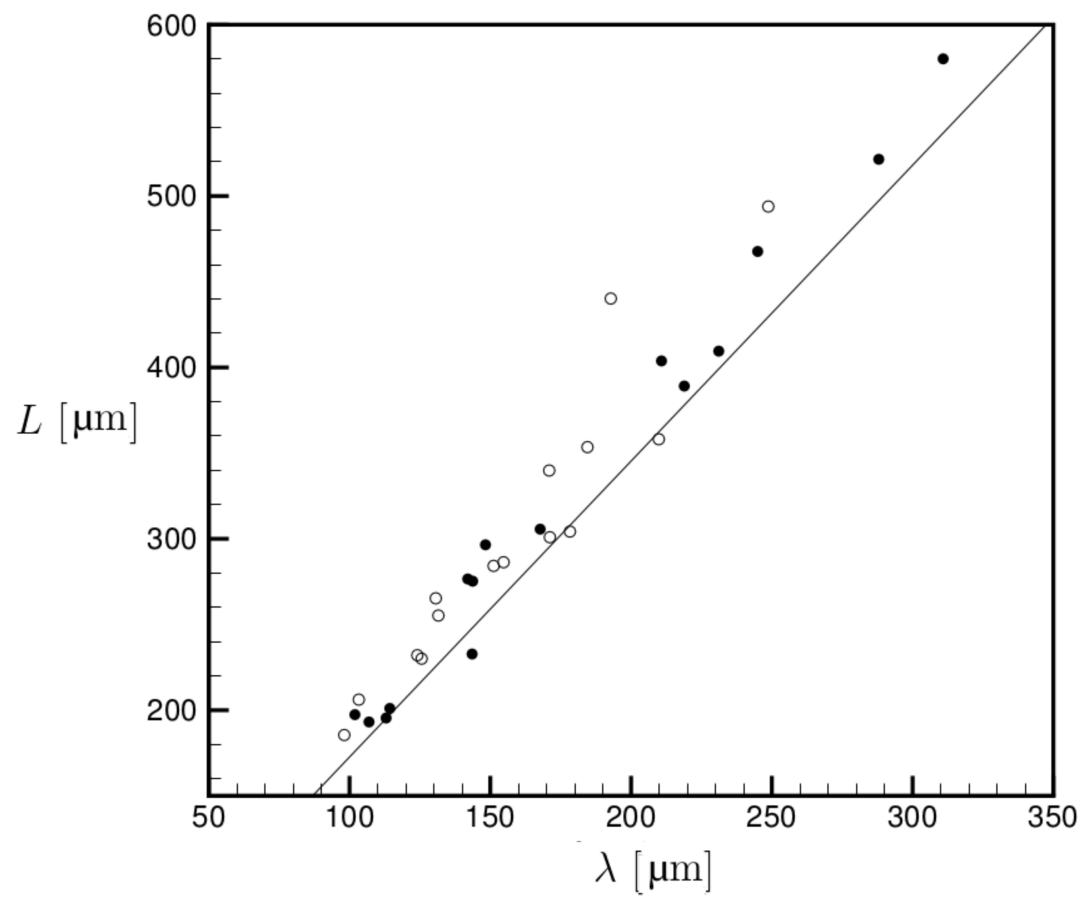

As shown in figure 3.6, our theoretical linear correlation given by eq. (3.5) was also validated experimentally. It is possible to see that its characteristic motion remains the same regardless the nematode size for a typical medium with $E \cong 2.6 \mathrm{kPa}$. Consequently, the wavelength responds linearly to the nematode size even in its larval stage. The remarkable agreement between our theory and the experimental measurements suggests that $C$. elegans uses indeed a sinusoidal locomotion. It is interesting to observe that this prediction was proposed considering that $L \sim 2 \lambda$, which was based on our previous experiments.

We may also compare the nematode's bending angle $\theta$ with the distance $R$ between head and tail. Considering a sinusoidal movement, the angle should reach its maximum at the smallest $R$ and the minimum angle when $R$ reaches its maximum. Figure 3.7 compares distance $R$ and angle $\theta$. 
Figure 3.7: Nematode's bending angle $\theta$ and head to tail distance $R$ (defined as $L / 2$ ) as a function of time considering the same trajectory. It is possible to observe at points $A, B, C$ and $D$ how these two variables relate. At $A$, the bending angle is high and the nematode's body curved. The same behavior is seen at $B$ and $C$. On the other hand, at $D$, the bending angle reaches its minimum and the nematode's body is fully streched. [Source: the author.]
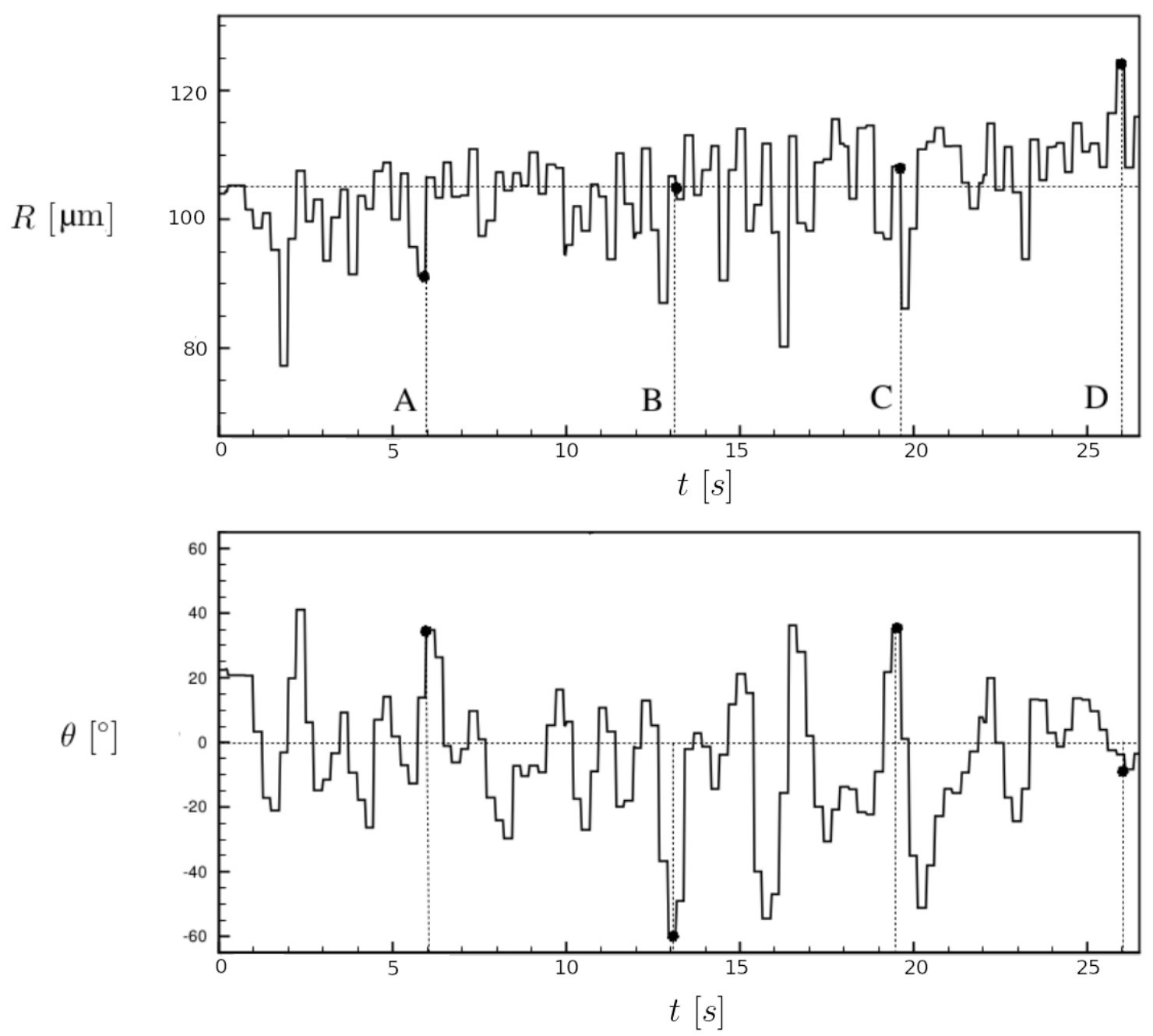

\subsubsection{Non-harmonic motion analysis}

Considering the scallop theorem, we may postulate that in order to move, a C. elegans has to conduct a non-harmonic motion. Analyzing the trajectory of the individuals, as presented in figures 3.8 and 3.9, we may observe a more complex non-harmonic oscillatory motion of the nematode. The head motion now is composed of different vibrational degrees of freedom in contrast with a 
simple harmonic motion. This condition breaks reversibility and it is necessary for the nematode to move and find food. However, the centroid trajectory is almost harmonic.

Figure 3.8: Trajectory of 10 starving nematode's centroid taken randomly from the sample. The trajectories were subtracted from their point of origin to start at $(x, y)=(0,0)$. [Source: the author.]

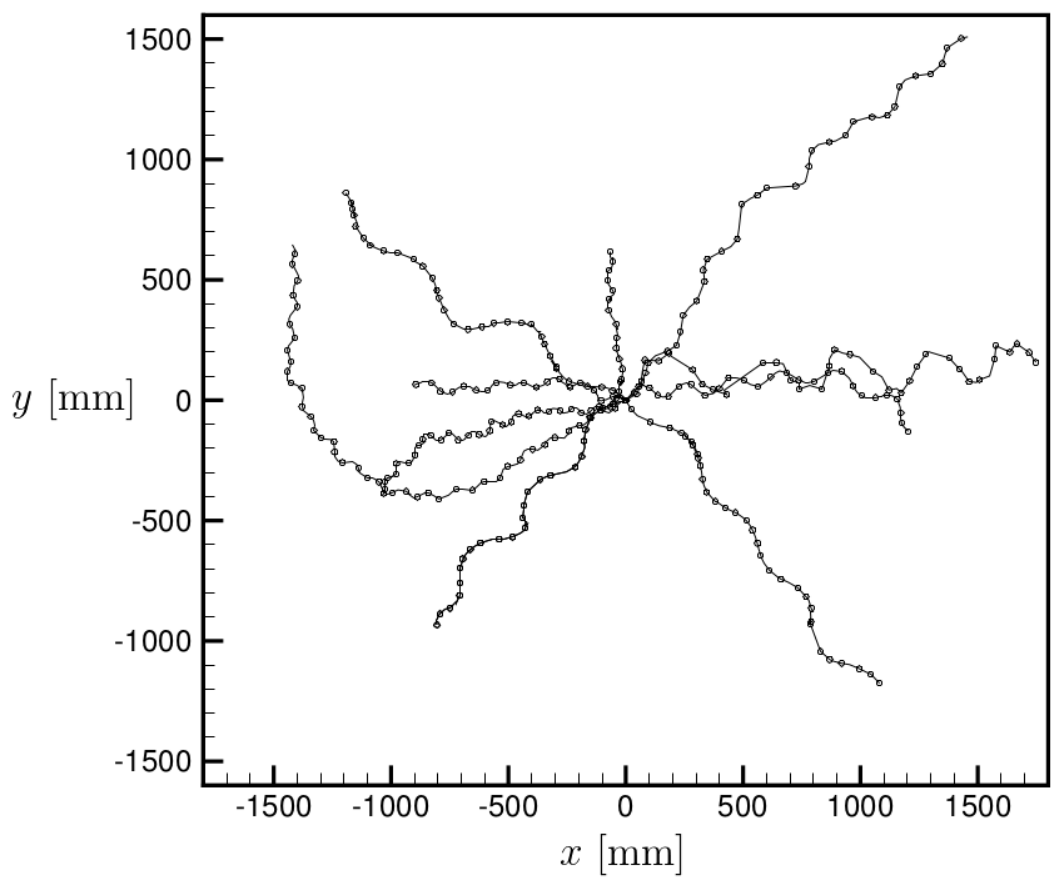

In the absence of food, the individuals tend to move faster and in different directions searching for food and produzing non-harmonic trajectories as depicted in figure 3.8. Moreover, the worms increase their velocity and tend to crawl higher distances. However, when a collony of bacteria is present, all individuals tend to crawl with similar velocities, i.e. proportional to the nematodes size. Under this condition, they also present fewer non-harmonic oscillations and do not explore the NGM plate, reducing its crawling path. In the frequency domain, we may understand how numerous modes of vibration are present in the complex nematode motion. The Fast Fourier Transforms were perfomed in Scilab using a script developed by the author and the results are shown in figures 3.10 and 3.11. There are typical differences between tail, head and centroid motions. The head presented a much more complex motion composed of several vibrational modes (degrees of freedom). We can notice that, there is a non-negligible energy in frequencies slightly higher than 2 . This is a direct consequence of the fact that the nematode head must command the rest of the body and 
Figure 3.9: Trajectory of 10 well-fed nematode's centroid. In this case, the individuals were taken randomly from the agar plate filled with $E$. coli. The trajectories were subtracted from their point of origin to start at $(x, y)=(0,0)$. [Source: the author.]

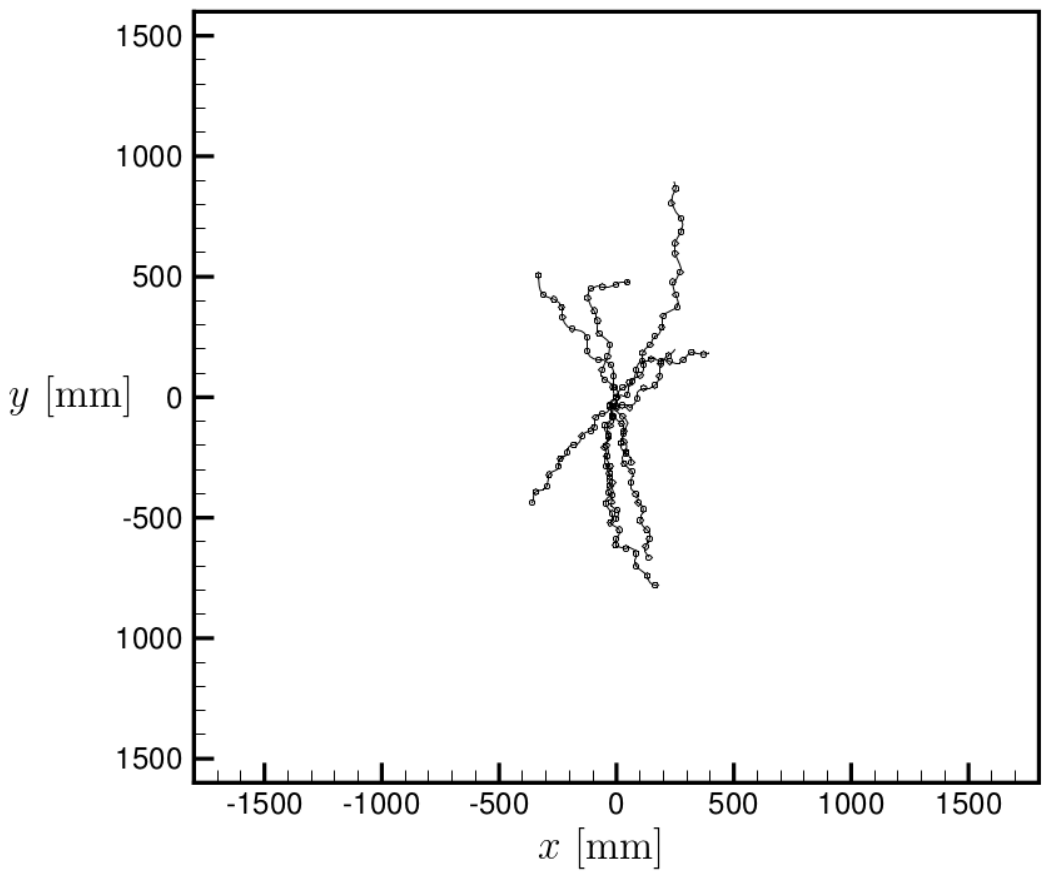

so it responds at higher frequencies and develops a complex "bending" motion in the absence of food. The centroid follows an almost harmonic pattern showing only one mode corresponding to the fundamental frequency i.e., having a minimum bending and effort. The thinning of the tail in relation to the rest of the nematode cylindrical body allows it to function similarly to a flagellum, presenting several motion modes and frequencies. Thus the kinematic reversibility is overcome. Considering that the starving nematodes tend to move faster and conduct a non-linear movement we may expect some broadband spectrum in the frequency domain. Under this condition, the nematode is searching for food and moving rapidly its head in different path lines. This motion transition of the nematode head in the absence of food corresponds to a dynamically non-harmonic response of the nematode trajectories in contrast with the nearly harmonically sinusoidal periodic motion observed under favorable conditions of food.

It is remarkable that the starving nematodes present a dominant frequency, which represents the majority of its motion characteristics. However, there is some spectral spreading taking energy 
from this principal frequency. In this condition, the nematode starts to move its body differently from an harmonic pattern. Although this motion may enable the nematode to move in creeping flow conditions, it may also hinder its translational motion. Thus the nematode must use some energy to bend its head in different directions.

Figure 3.10: Comparison of head (a) and centroid (b) motion of one specific individual of the starving sample with adjusted range. The dashed line shows the energy of the second harmonic. [Source: the author.]

(a)

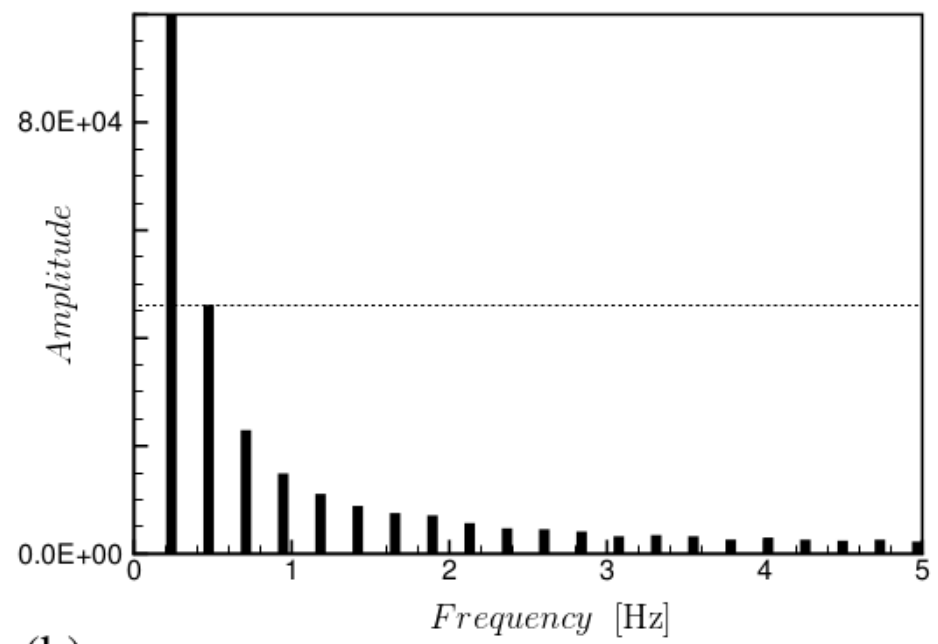

(b)

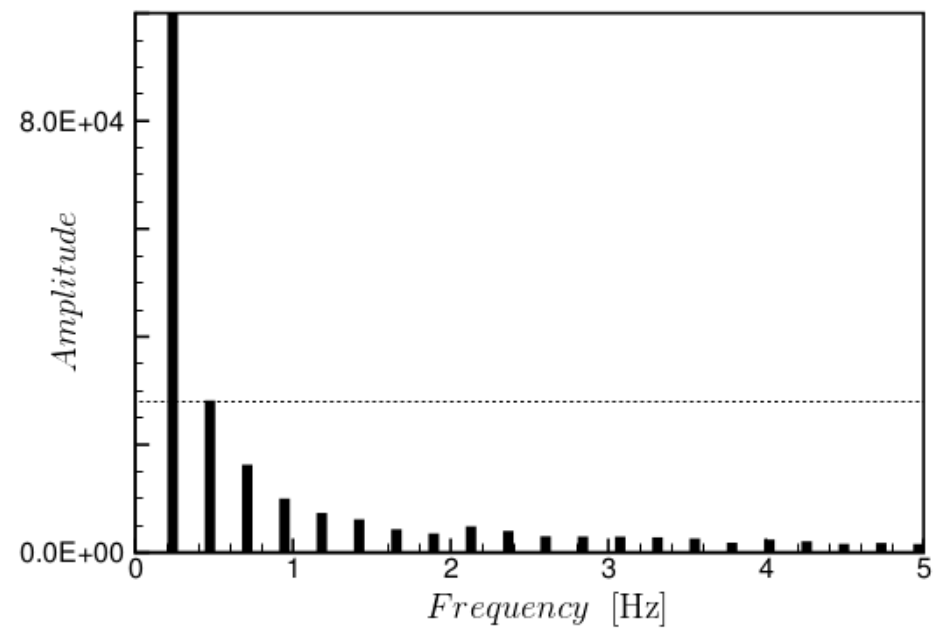

Figures 3.10 and 3.11 show the difference from head and centroid trajectory FFT from individuals taken from starving and colonized plates, respectively. When food is available, all nematodes follow a very similar behavior following the E. coli colony. However, observing figure 3.11, 
Figure 3.11: Comparison of head (a) and centroid (b) motions of one specific individual of the well-fed sample with adjusted range. The dashed line shows the energy of the second harmonic. [Source: the author.]

(a)

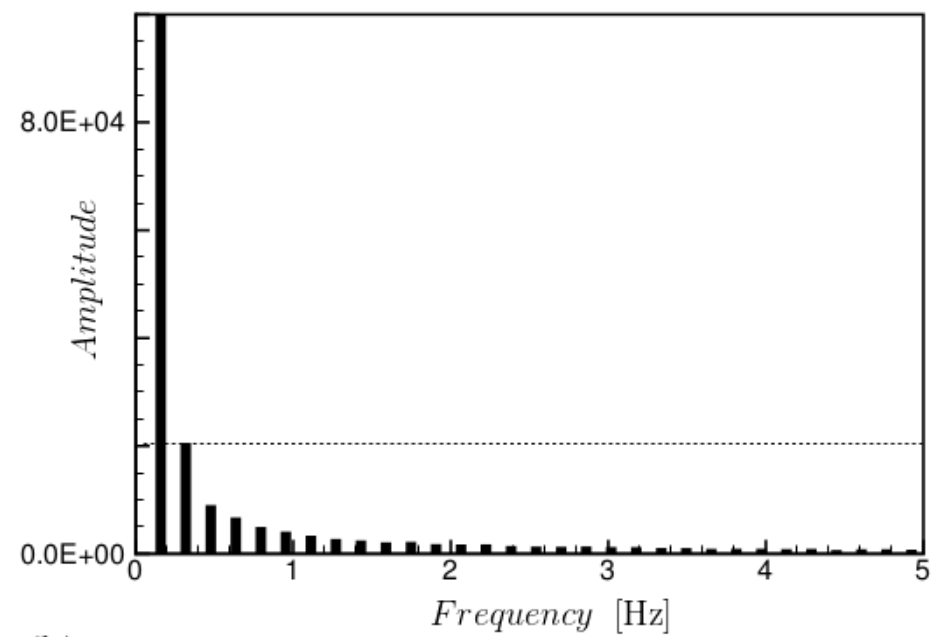

(b)

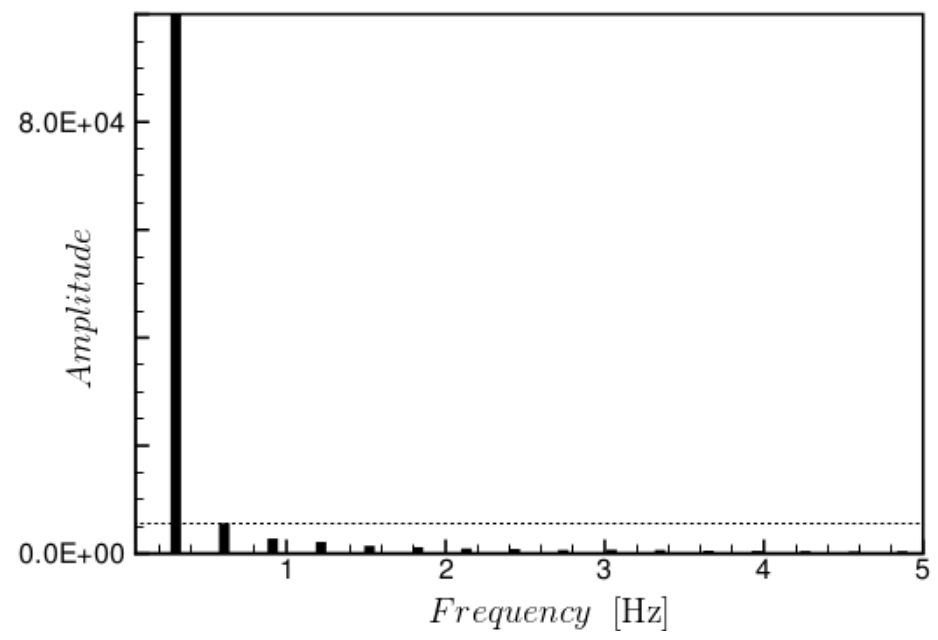

we may conclude that the motion of the head is quite different from the centroid trajectory. This behavior is directly associated with the nematodes velocity and size, which can change significantly depending on their physical and chemical characteristics.

We may observe in figure 3.10 that the starving individuals present a spectrum with a more continuous distribution of modes of this complex oscillatory motion. Here, they show that the energy in each frequency is much higher than the energy shown in figure (3.11), for example. As 
shown before, when bacterial collonies are present, the nematodes tend to move slower and in well-defined harmonic sinusoidal trajectories. Under this condition the trace left behind the nematode seems to be very similiar to a sinusoidal wave and the slip coefficient should be close to zero. However, when crawling in free surface (NGM without bacteria colonies), considering greater slip, individuals activate the "global search" mechanism, increasing their velocities, bending and constantly changing their trajectories.

It is also interesting to notice that the nematode's extremities excitation has its origins in viscous effects, since its head and tail describe a highly nonlinear motion with much more vibrational modes than its body. On the other hand, when the nematode is hungry, seeking for food, its excitation is more influenced by biological and physiological aspects. In this regime, the development of different vibrational modes is more complex, since now the animal body presents a non-harmonic motion. This can be confirmed by the comparison of typical trajectories in the presence and absence of food.

\subsection{Chapter conclusions}

In this chapter we have presented a statistical analysis on the kinematics-wave motion of a suspension of $C$. elegans in a gel-like medium. We have studied two different populations from a biologial perspective of the surrounding medium. These populations consisted in a starving and a well-fed population of nematodes. We have found experimentally a linear correlation between the length and the wavelength of the individuals for both populations. We proposed a theoretical correlation to justify this linear dependence. The results have indicated that $C$. elegans indeed uses sinusoidal propulsion to move in creeping flow.

We have also found that, whereas the centroid of the individuals in both populations behaves nearly harmonically, their heads and tails evolve to highly non-harmonic motion. This nonlinear motion is used to break the time reversibility in which they are trapped due to their small sizes, known as kinematic reversibility in low Reynolds number flows. Another important finding of the present work is the discrepancy observed in the collective motion of both populations. We observed that well-fed individuals tend to move in the direction of E. coli collonies with less spreading in the surrounding medium. On the other hand, a starving population collectively behaves quite differently, seeking for food in several possible directions and with a much stronger head motion. 
In the next chapter, the rheology of the active suspension will be analyzed. 


\section{Rheological Analysis}

\subsection{Experimental Procedure}

In the suspended fluid, large quantities of $C$. elegans can be cultivated depending on the concentration of $E$. coli as food source. Under conditions of plentiful food and low population density, larvae pass through four larval stages (L1, L2, L3, and L4) before molting into reproductive adults. It has been observed in previous studies that the presence of the bacteria severely modifies the kinematic behavior of the nematode (MALVAR et al., 2017). In addition, the development of these worms is closely linked to the presence of food in the substrate. However, during a short time window, L1 larvae select one of two alternative developmental pathways in response to environmental conditions (BAUGH, 2013). When little food is available, nematodes still exhibit the standard sinusoidal kinematic behavior, but L1s enter the diapause and arrest development as dauer larvae. Dauer formation requires energy and, therefore, the presence of food in the environment. For this reason, all experiments were performed with a small amount $(\sim 0.1 \%)$ of $E$. coli in the suspension. Thus, the nematodes were kept in the dauer stage with an average diameter of $17 \mu \mathrm{m}$ at standard kinematic behavior. Although the presence of bacteria could influence the rheological chacteristics of the medium, this behavior is irrelevant due to the low concentration of E. coli and the size difference between bacteria and the nematodes, which have approximately $17 \mu \mathrm{m}$ of diameter and $300 \mu \mathrm{m}$ of lenght.

The protocol we used to produce the liquid medium was proposed by Stiernagle (STIERNAGLE, 2006) and consists on producing $S$ Basal composed of $2.92 \mathrm{~g}$ of $\mathrm{NaCl}, 0.5 \mathrm{~g}$ of $\mathrm{K}_{2} \mathrm{HPO}_{4}$ and $3 \mathrm{~g}$ of $\mathrm{KH}_{2} \mathrm{PO}_{4}$ dissolved in $400 \mathrm{ml}$ of $\mathrm{ddH}_{2} \mathrm{O}$. Each component was separately autoclaved and mixed using sterile technique. After that, $1 \mathrm{ml}$ of cholesterol $(5 \mu \mathrm{g} / \mathrm{ml}$ dissolved in $100 \%$ ethanol) and $\mathrm{H}_{2} \mathrm{O}$ to 1 liter were added. Sterilized 1 mol potassium citrate $p H 6$ ( $2 \mathrm{~g}$ of citric acid monohydrate and $29.4 \mathrm{~g}$ of tri-potassium citrate monohydrate dissolved in $70 \mathrm{ml}$ of $d d \mathrm{H}_{2} \mathrm{O}$ ), $1 \mathrm{~mol} \mathrm{CaCl}\left(7.35 \mathrm{~g}\right.$ of $\mathrm{CaCl}_{2} \cdot \mathrm{H}_{2} \mathrm{O}$ dissolved in $40 \mathrm{ml}$ of $\left.\mathrm{ddH}_{2} \mathrm{O}\right), 1 \mathrm{~mol} \mathrm{MSO}_{4}$ and trace metals $100 \times$ were mixed to the original suspension. Trace metals solution was produced adding $0.186 \mathrm{~g}$ of disodium EDT A, $0.069 \mathrm{~g}$ of $\mathrm{FeSO}_{4} \cdot 7 \mathrm{H}_{2} \mathrm{O}, 0.02 \mathrm{~g} \mathrm{MnCl}_{2} \cdot 4 \mathrm{H}_{2} \mathrm{O}, 0.029 \mathrm{~g} \mathrm{ZnSO} \cdot 7 \mathrm{H}_{2} \mathrm{O}$ and

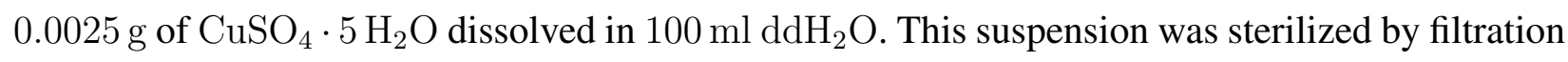
on $22 \mu \mathrm{m}$ and protected from light. 
In order to produce the concentrated $E$. coli OP50 shown in figure 4.1, $2 \mathrm{ml}$ of LB medium with a colony of freshly streaked OP50 was stored at $37^{\circ} \mathrm{C}$ for 8 hours. From this culture, $4 \times 0.5 \mathrm{ml}$ was inoculated in $4 \times 500 \mathrm{ml} \mathrm{LB}$ in 21 Erlenmeyer. After 18 hours of growth procedure at $37^{\circ} \mathrm{C}$, the suspension was spun at $8 \times 250 \mathrm{ml}$.

The final liquid medium concentration was obtained using the following protocol. Using sterile techniques, $45.7 \mathrm{ml}$ of $\mathrm{S}$ medium, $50 \mu \mathrm{l}$ of cholesterol $5 \mathrm{mg} / \mathrm{ml}, 500 \mu \mathrm{l}$ of $1 \mathrm{~mol}$ potassium citrate $p H 6,500 \mu \mathrm{l}$ trace metals $\times 100,150 \mu \mathrm{l} 1 \mathrm{~mol} \mathrm{CaCl}_{2}$ and $150 \mu \mathrm{l} 1 \mathrm{~mol} \mathrm{MgSO}_{4}$ were mixed. A pellet of OP50 (corresponding to $150 \mathrm{ml}$ of overnight culture) was added. Using a total of $3 \mathrm{ml}$ of S medium, starving worms were collected from the plates and added to the culture medium. The suspension shown in figure 4.1 was incubated at $22{ }^{\circ} \mathrm{C}$ and vigorously shaked for 3 days.

Figure 4.1: Cultivation steps of both studied liquids. scv[Source: the author.]

(a) Solution of E. coli.

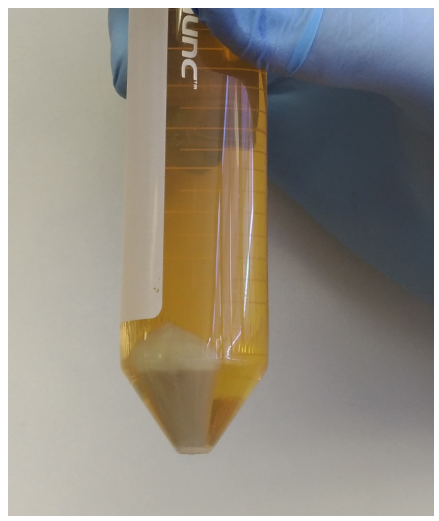

(b) Suspension of nematodes.

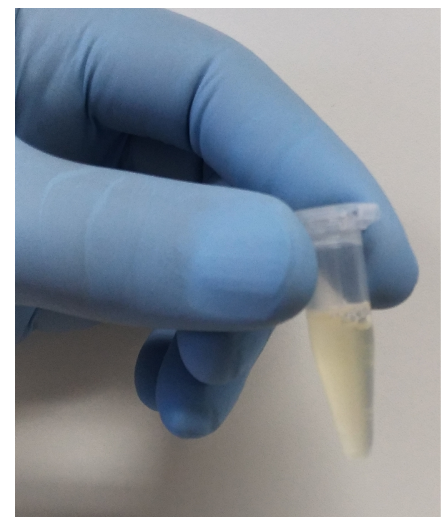

The rheological properties of the nematode suspension in liquid medium was determined experimentally using an Anton Paar rheometer (Physica model MCR 301 in the Laboratory of Microhydrodynamic and Rheology - VORTEX - University of Brasília). This rotating disks rheometer can perform rheology in oscillatory shear experiments, which are very useful to achieve small shear rates (i.e. linear viscoelastic regime). Its minimum torque is $0.1 \mu \mathrm{N} \cdot \mathrm{m}$ during steady shear and $0.02 \mu \mathrm{N} \cdot \mathrm{m}$ during direct strain oscillation method, which provides the ability to control oscillatory strains as low as $0.1 \mu \mathrm{rad}$ - important for studying material with delicate structures - with a torque resolution of $0.001 \mu \mathrm{N} \cdot \mathrm{m}$.

It is important to highlight that all samples also contain E. coli colonies which modify the rheology of the suspension. However, the rheology of suspensions of bacteria was already studied and its proprerties are well known. 
The experimental data were obtained using the software Rheoplus from Anton Paar, which define the experiment conditions such as strain, oscillatory frequency and temperature gradient. The tests were based on isothermal small deformation oscillatory shear. Considering a gap of $0.08 \mathrm{~mm}$ and using a typical strain of $\gamma=0.4$, three experiments were carried out in 7 different samples with different volume fractions $(0.1 \leq \phi \leq 3.5 \%)$, in order to observe the active suspension collective behavior, as can be seen by the suspension microstructure presented in figure 4.2.

Figure 4.2: Three typical suspension selected during the experiments From left to right $\phi=$ $0.9 \%, 1.5 \%$ and $2.1 \%$. [Source: the author.]
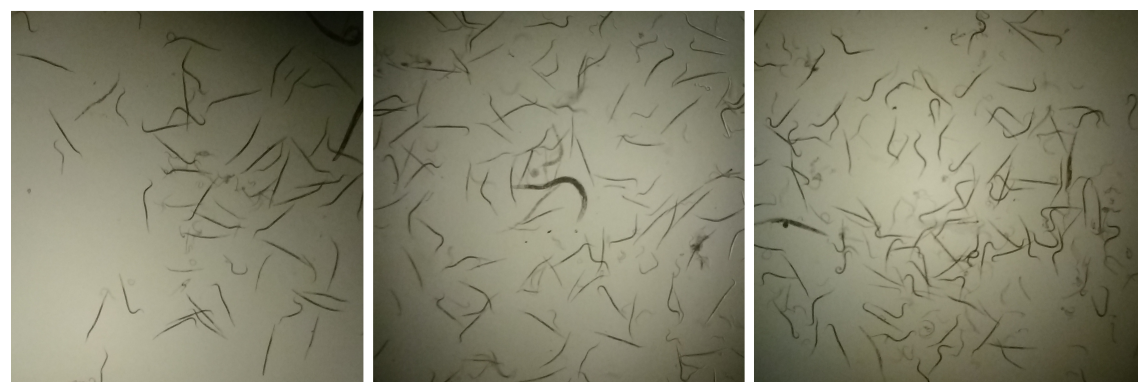

\subsection{Results and discussions}

\subsubsection{Comparison between active and passive suspension}

The first investigation is based on the comparison of the elastic modulus $G^{\prime}$ for different volume fractions of $C$. elegans with a $1 \%$ passive suspension of aqueous solution of polyacrylamide. Figure 4.3 shows the elastic modulus as a function of the volume fraction of the active particles. In the insert, we can also see the absence of a shear elastic modulus as the frequency goes to zero for the polyacrylamide suspension (i.e. $G_{0}^{\prime}=0$ ). The main objective is to show how the presence of active particles affects the bulk behavior of a viscoelastic suspension. However, we are not only interested in the activity of the nematode, but also in how its morphological characteristics affect the suspension in general. In other words, the polyacrylamide solution behaves like a simple Maxwell fluid. On the other hand, in the active suspension, besides the non-monotonic behavior of the elastic modulus, there is a non-null shear elastic modulus at low frequencies associated with the intrinsic movement of the active particle, which inserts a solid-like behavior even at low volume fractions. This is also seen in emulsions subjected to viscoelastic regimes for volume fractions above the spherical drops maximum random packing ( $>$ 64\%) (OLIVEIRA AND CUNHA, 2011). In the case 
of nematodes, this elastic intrinsic behavior is mainly related to their bending. As the active particle increases its curvature, there is an increase in the restoring mechanism. Thus, the bulk viscous dissipation is altered by the force exerted by the active particle on the ambient fluid.

Figure 4.3: Shear elastic modulus, $G^{\prime}(\omega \rightarrow 0)$, and loss modulus, $G^{\prime \prime}(\omega \rightarrow 0)$, as a function of the volume fraction. The insert shows $G^{\prime}(\omega)$ of an aqueous polyacrylamide solution at $\phi=1 \%$.

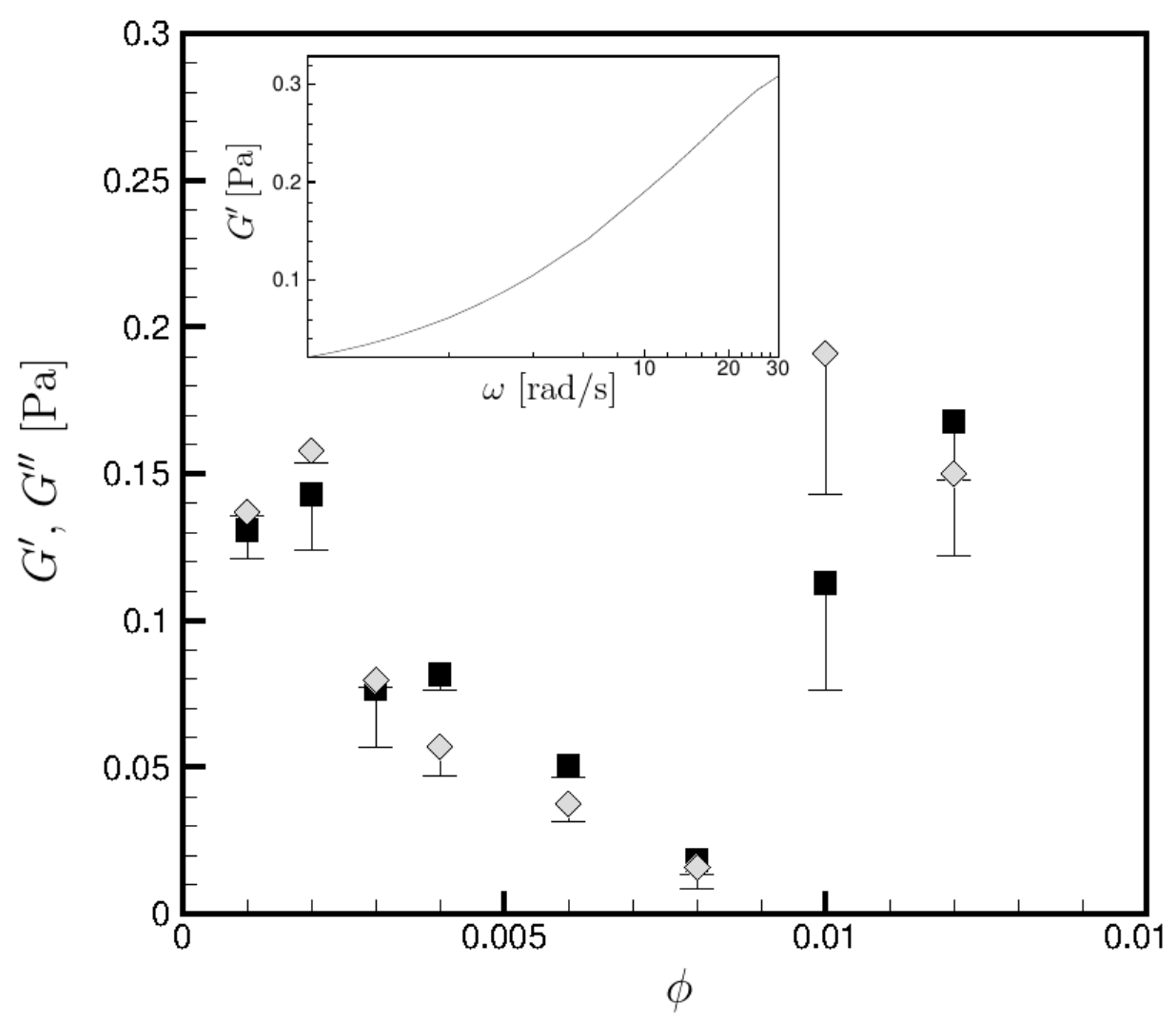

In the equilibrium condition the active particle is also not deformed (i.e. not aligned in the flow direction) by the flow, because the flow is very week and no shear thinning is observed. However, there is a non-equilibrium condition induced by the active particle motion. The bulk effect of this bending inhibits stretching and can be associated with an aparent dynamic yield stress at low shear rate. This effect introduces an elasticity in the bulk suspension and produces a $G^{\prime}$ different from zero even as the frequency goes to zero. So, this active suspension cannot be described by a standard Maxwell model in which $G_{0}^{\prime}=0$. In addition, for some volume fraction, the entanglements of nematodes lead to $G^{\prime}>>G^{\prime \prime}$. In contrast, in polymeric suspension at very low strains, the macromolecules are not being deformed and there is no elastic effect. In this condition, the 
macromolecule is fully relaxed at an equilibrium condition (i.e. randomly coiled). There is a very interesting finding here. At volume fractions below the critical (in this case close to 0.004) the effect of yield stress is higher and it will produce a kind of plastic viscosity, which is the resistance to deformation after the yielding point.

Using the empirical result obtained by Yasuda, Armstrong \& Cohen (YASUDA et al., 1981), which states that $N_{1} \sim\left(2 G^{\prime}\right)_{\dot{\gamma}=\omega}=\sigma_{11}-\sigma_{22}$ (i.e. first and second normal stresses differences) for the limit of small shear (in steady rates) and frequency (oscillatory shear), we can figure out when the collective behavior effect of the living particles becomes important to the rheology. Laun's rule (LAUN, 1986), which is a correction of this first result, states that the first normal stress difference from the shear elastic modulus at low frequency. It is important to highlight that this is valid only when in the limit of low frequency, low shear rate (AL-HADITHI et al., 1992a) and when $\left(G^{\prime} / G^{\prime \prime}\right)^{2}<<1$ so that

$$
\begin{aligned}
& \lim _{\omega \rightarrow 0} \frac{G^{\prime}(\omega)}{\omega^{2}}=\lim _{\dot{\gamma} \rightarrow 0} \frac{N_{1}(\dot{\gamma})}{2 \dot{\gamma}^{2}} . \\
& \left.\frac{G^{\prime}(\omega)}{\omega^{2}}\right|_{\omega \rightarrow 0}=\left.\frac{N_{1}(\dot{\gamma})}{2 \dot{\gamma}^{2}}\right|_{\dot{\gamma} \rightarrow 0} .
\end{aligned}
$$

Figure 4.4 shows the first normal stress difference for $\phi=3.5 \%$. The insert presents the difference $\Delta N_{1}=N_{1}(\omega \rightarrow 2.5)-N_{1}(\omega \rightarrow 0)$ for different volume fractions of active suspensions. The first normal stress difference increases only for volume fractions above $3 \%$, as the active particles form structures that can be stretched have a tendency to align in the flow directions. Therefore, our experiments have indicated that the orientation effect of an individual active particle on the bulk rheology is not perceptible at concentrations below $3 \%$. For $3.5 \%$, however, there is a considerable variation of the first normal stress of about $100 \%$. We attribute this variation to the stretching imposed by flow on the active particle like-structures which in turn produces anisotropy in the flow due to the presence of deformed-oriented structures. For macromolecules suspensions at small shear rates, $N_{1} \propto \dot{\gamma}^{2}$, (GUPTA, 2010). We observed that in the active suspension, $N_{1} \propto \dot{\gamma}^{3 / 2}$.

Figure 4.5 shows an remarkable difference between the rheological behavior of the active suspension examined here and a typical passive polymeric suspension. As expected, there is an 
Figure 4.4: Dimensional first normal stress difference obtained through Cox-Merz rule $\phi=3.5 \%$ as a function of the shear rate $\dot{\gamma}$. The insert shows the difference in $\Delta N_{1}=N_{1}(\omega \rightarrow 2.5)-N_{1}(\omega \rightarrow$ $0)$.

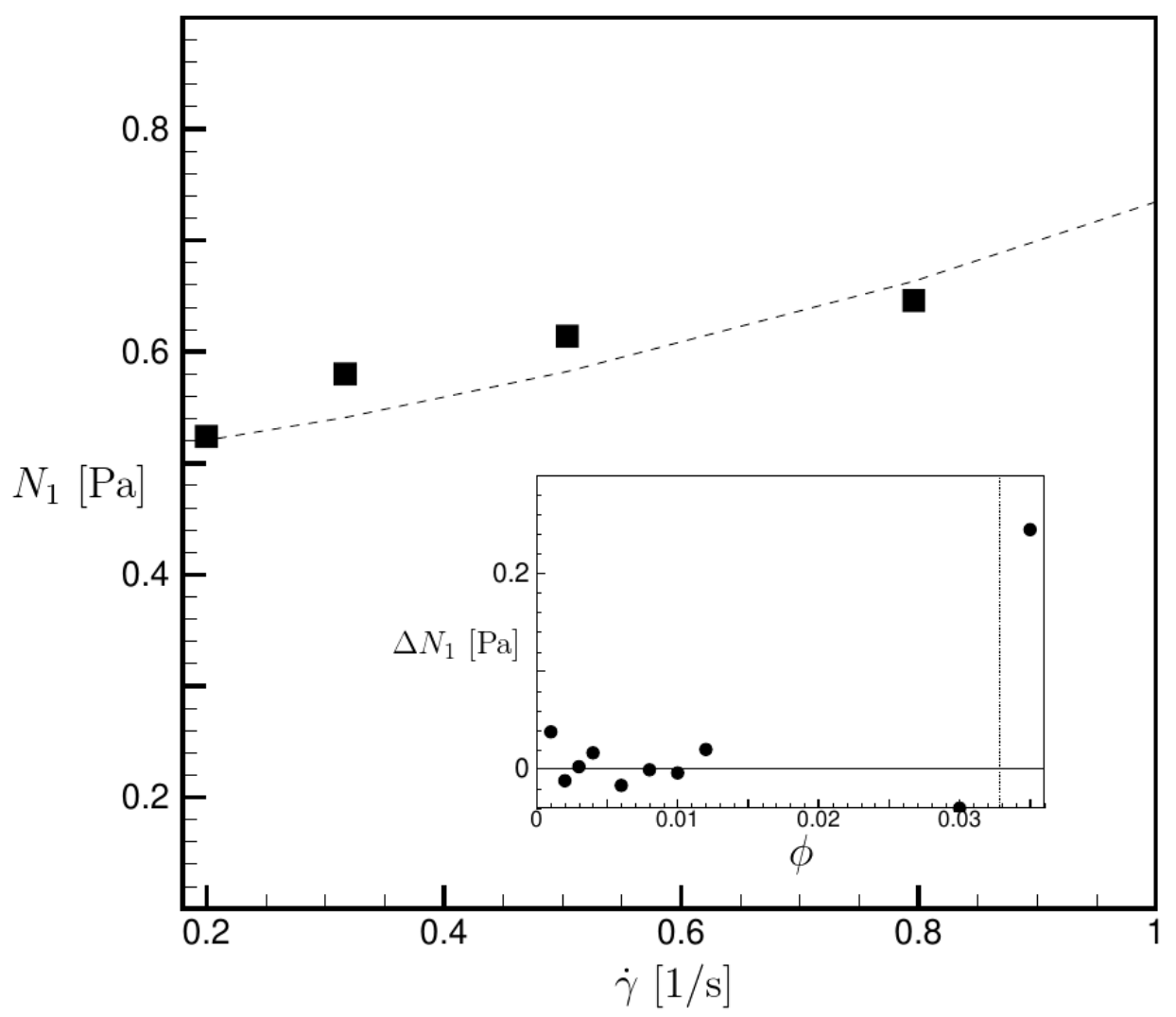

increase of $\sigma_{11}$ in the polyacrylamide suspension, due to the high stretching of the macromolecules that tend to be aligned in the flow direction even in a very dilute regime $(\phi<<1 \%)$. The increase of $\sigma_{11}$ only at higher volume fractions is a distinctive behavior of an active suspension where the interactions and the collective activity of the active particle, stretching and orientations of the alive structures become relevant. In this condition, the structures formed by the agglomerates of $C$. elegans and their interactions stretch. However, since the microorganism has an internal elasticity, the bending and relaxation actions of its body do not allow it to stretch and coil the same way as macromolecules do undergoing flow, and their moving behavior is not easily disturbed.

As observed before, when the shear rate increases, the active particles tend to decrease their transversal fluctuations and the structures align with the flow direction due to the applied stresses. Under this condition, there is a decrease in the effective viscosity. Moreover, Berri et al. (BERRI AND ET AL., 2010) observed that the continuous adaptability of the nematode locomotion is also 
Figure 4.5: Non-dimensional first normal stress difference obtained through Cox-Merz rule for $C$. elegans suspension of $\phi=1 \%$. The insert shows $N_{1}$ for an aqueous polyacrylamide solution of $\phi=1 \%$. [Source: the author.]

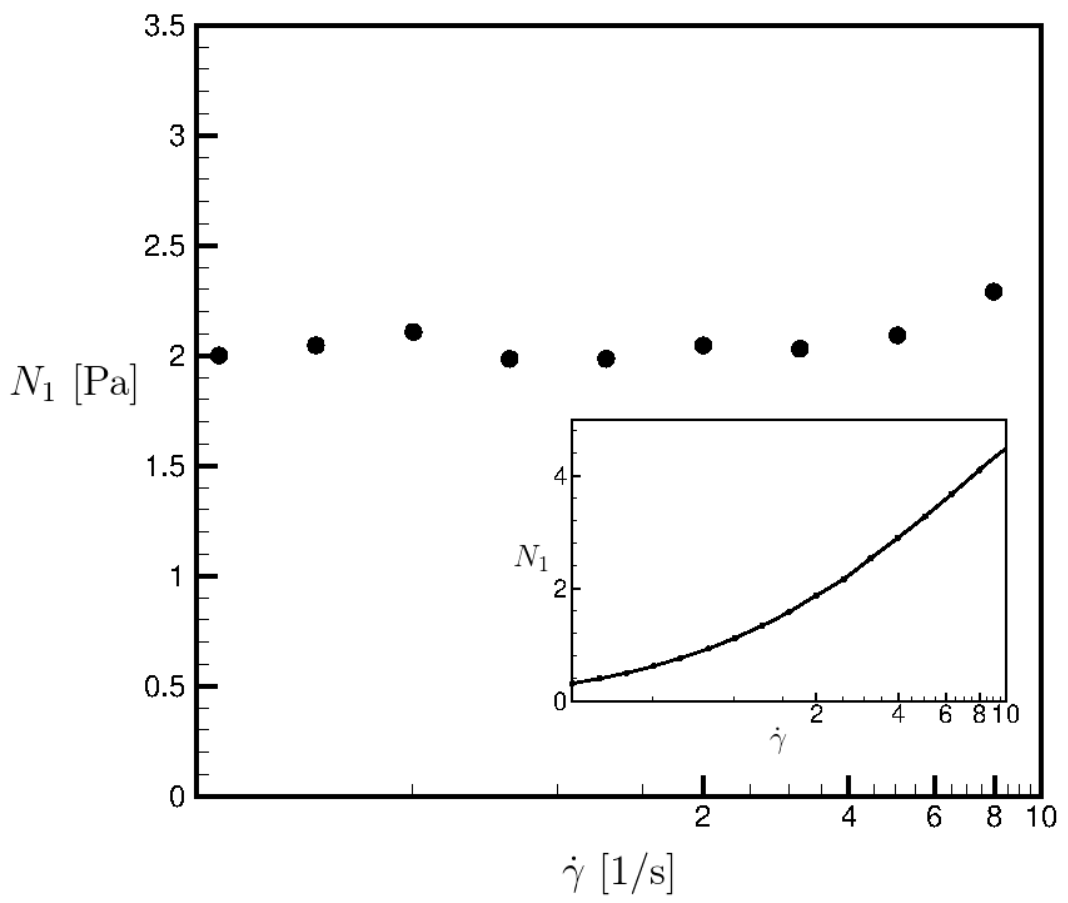

related to the external mechanical resistance. Gradually increasing external mechanical resistance on a swimming worm induces a continuous transition of locomotory gait, gradually decreasing the wavelength and frequency of undulations until the active particle gait resembles that of crawling on agarose surfaces (FANG-YEN et al., 2010).

\subsubsection{Effective viscosity for different volume fractions}

Considering the intrinsic motion of nematodes, we must observe that there is a disturbance in the flow even in the diluted regime. These instabilities can generate anomalous behaviors. For example, the effective viscosity can decrease depending on the volume fraction of microorganisms. This observation was previously made by Hatwalne et al. (HATWALNE et al., 2004), who generalized kinetic equations for liquid crystals to model the rheology of active suspensions. More recently, Ishikawa and Pedley (IsHIKAWA AND PEDLEY, 2014) performed Stokesian dynamics simulations 
of suspensions of spherical "squirmers", which swim as a result of a prescribed slip velocity on their surface. In the dilute limit, they found no change in effective viscosity due to swimming (for non-bottom heavy particles), a consequence of the spherical shape they assumed, which results in an isotropic orientation distribution (S AINTILLAN AND SHELlEy, 2011).

Normally, as multi-particle interactions begin to dominate the individual effect, higher order terms appear (i.e. virial expansion having terms $\phi^{2}, \phi^{3}$ and so on) as calculated by Batchelor (BATCHELOR, 1977). Krieger and Dougherty (KRIEGER AND DOUGHERTY, 1959) proposed a semi-empirical correlation for the effective viscosity of solid-liquid suspensions which is valid in the full range of particle volume fraction function, namely

$$
\eta_{r}=\left(1-\frac{\phi}{\phi_{\max }}\right)^{-[\eta] \phi_{\max }}
$$

where $\eta_{r}$ is the effective viscosity, $\phi$ is the particle volume fraction, $\phi_{\max }$ is the maximum packing and $[\eta]$ is the intrinsic viscosity. At higher $\phi$, the interaction between particles causes a stronger $\phi$ dependence and additional mechanisms become pronounced. This physical phenomenon involves a competition between shearing and thermal forces, in which the size of the particles plays a determining role (MARON AND PIERCE, 1956).

Mooney (MoOney, 1951) proposed a useful functional form describing the dependence of relative viscosity on particle volume fraction. An exponential dependence for semi-diluted suspensions directly related to the particle's geometry was given as

$$
\eta_{r}=\exp \left[\left(\frac{[\eta] \phi}{1-\phi / \phi_{\max }}\right)\right]
$$

Mooney's equation can be modified (DUNKEL et al., 2013) in order to compute the complex behavior induced by active particle fluctuations (BRATANOV et al., 2015) and crowding behavior (WENSINK et al., 2012). So we have analyzed each region shown in figure 4.6 based on both approaches (i.e. equations 4.3 and 4.4) in order to fit our experimental results. It is import to highlight that this first approach does not compute the deformation of each particle. As a matter of fact, this proposition is valid only when an equivalent sphere diameter is considered, that is, when the active 
particle is involved by a bounding spherical particle.

Figure 4.6: Effective viscosity $\eta_{r}$ as a function of $\phi$, for a suspension of wild type (N2) C. elegans. The pure anisotropic region (1), the active elastic region (2) and the structure formation region (3) are divided, showing the respective critical values of $\phi$. [Source: the author.]

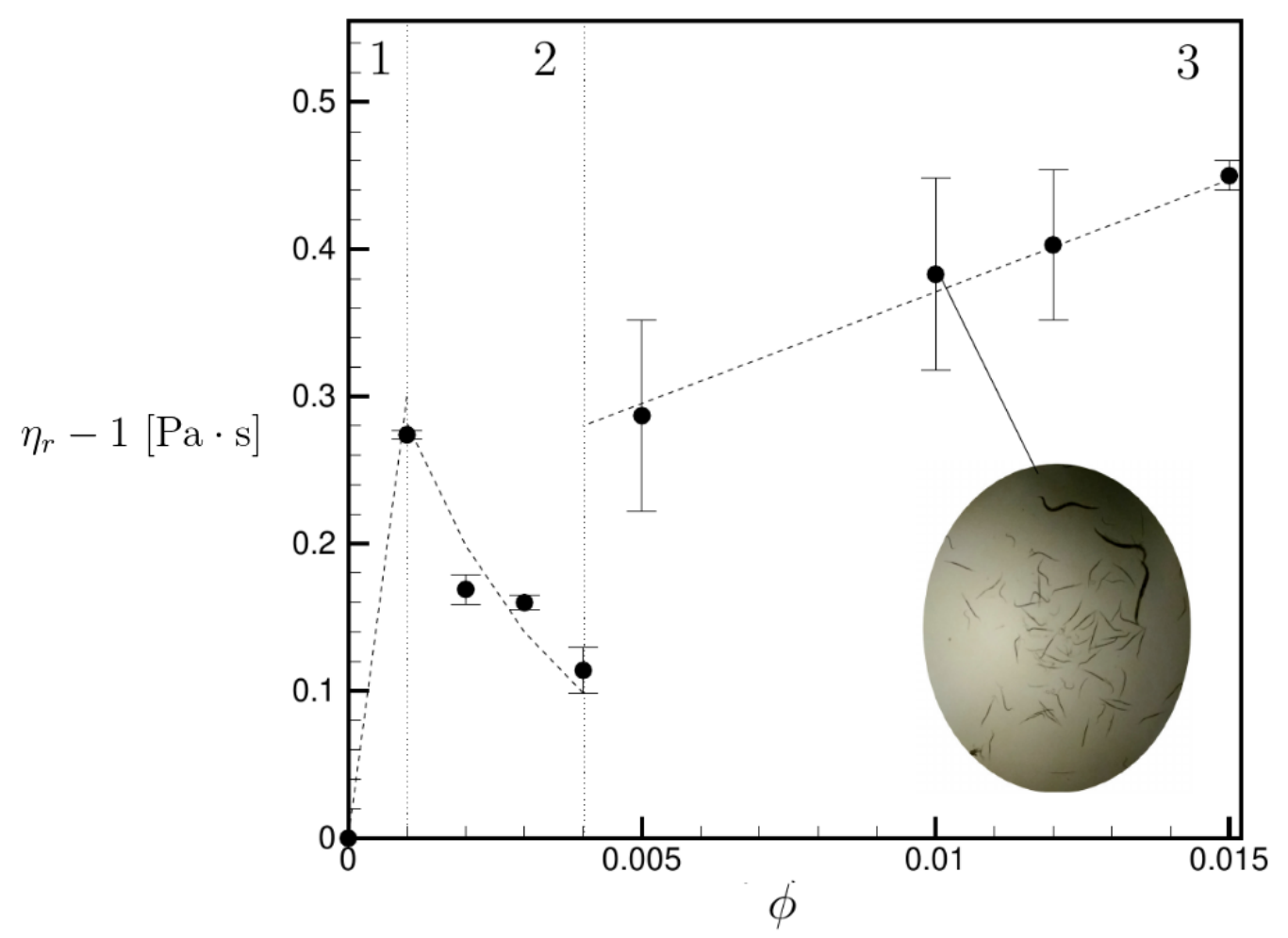

The effective viscosity of a suspension is a result of the total shear stress induced by the applied shear flow and internal fluctuations. The intrinsic resistance of the suspended fluid related to the active particle motion is directly related to the suspension viscosity. The effective viscosity related to the presence of the active particles as a function of the volume fraction is shown in figure 4.6. We observe three different regimes.

1. Pure anisotropic region - $0<\phi<0.002$ : Ttheoretically, in this region, this liquid would behave similarly to other very dilute suspensions. However, more experiments would be needed to corroborate these claims. It corresponds to the very diluted regime, where the effective viscosity presents a linear behavior as $\phi$ increases. In suspension regime, the non-dimensional effective viscosity would be given by $\eta_{r}=1+K n \ell^{3}$, where $K$ is an experimental constant, 
$n$ is the density number and $2 \ell$ the length of the rod-like active particle (BATCHELOR, 1977). In terms of the particle volume fraction, we have:

$$
\eta_{r}=1+\left(\frac{K}{\pi}\right) \phi\left(\frac{\ell}{a}\right)^{2}
$$

where $a$ is the rod-like active particle radius and $\ell / a$ is the particle aspect ratio. Thus, in a very diluted regime, we have a Batchelor rod like effect that would be equivalent to an Einstein's suspension of spheres with radius $\ell$ (EINSTEIN, 1905). At these very lower volume fractions the particle activity relaxation time is much smaller than a flow time scale, rendering this effect imperceptible. Therefore under condition of very dilute active suspension, the average particle stresslet (particle stress) does not contribute with elastic effect to the suspension bulk stress.

2. Active elastic region $-0.002<\phi<0.004$ : There is a decrease in the bulk viscosity in a non-linear fashion. That means that the elastic effect of each particle has already become important. In this particle volume fraction range, particle activity relaxation time is comparable to the applied flow time scale. In other words, the non-dissipative energy production results in negative viscosity values and the particle stresslet decreases. This occurs typically with pusher particles like the nematodes investigated here in the sense that they repel fluid from the body along their axis and draw fluid to their sides. Although the volume fraction is still low, the particle activity and its elastic bending becomes more important than the pure viscous anisotropic effect described for lower concentrations. Thus, this first transition is most closely linked to the elasticity of the nematodes. The effective viscosity in terms of volume fraction is:

$$
\eta_{r}=1+\exp \left(\frac{\beta \phi}{1-\phi / \phi_{\max }}\right)
$$

where $\beta$ is an important parameter which characterizes the strength of the elementary particle force dipole exerted on the solved fluid and its sign has vital rheological consequences. The particle activity enhances effective fluid viscosity for $\beta>0$ (i.e. puller microorganisms) and reduces it if $\beta<0$ (i.e. pusher). This active component comes from a swim stress, which is analogous to the osmotic Brownian stress of passive particles (TAKATORI AND BRADY, 2014). 
3. Structure formation and particle interaction $-\phi>0.004$ : The effective viscosity increases again and assumes positive values. That is probably related to the particle velocity fluctuations produced by viscous hydrodynamic interaction of the neighboring anisotropic particles over a test particle. The collective effect appears due to the particle-particle interaction and to the tendency to align in a common direction, producing the increase of the suspension relative viscosity. In this region, we may write the effective viscosity as a virial expansion:

$$
\eta_{r}=1+c_{1} \phi+c_{2} \phi^{2},
$$

where $c_{1}$ and $c_{2}$ are specific coefficients related to the active suspension. The third term of this equation is explicitly related to the interactions between two active particles.

Lopez (LOPEZ et al., 2015) showed that the collective motion of swimming bacteria can lower the viscosity of a fluid to zero, mimicking a superfluid like liquid helium. For highly active bacteria, the viscosity can even become negative, meaning the swimmers are pushing the fluid along. In the same way, C. elegans can lower the liquid viscosity by aligning themselves such that their pushing contributes to the velocity gradient. However, contrary to what was observed in suspensions solely composed of bacteria, in the case of suspension of $C$. elegans, this decrease is not enough to decrease $\eta_{r}-1$ to negative values. This is directly related to entanglements and size of the nematode, which, however, may decrease the bulk viscosity of the suspension as a function of the volume fraction. Potomkin (РотомкIN et al., 2017) demonstrated how the flexibility of bacterial flagella affects macroscopic properties of suspension of microswimmers. They found that flagellar bending may lead to a decrease of the effective viscosity in the absence of random reorientations, which is very similar to the behavior observed in C. elegans' suspension.

\subsubsection{Stress relaxation function and particle activity relaxation}

Although most experimental measurements and theoretical models have focused on the steady flow rheology, we anticipate the competition between flow alignment and orientational relaxation by run-and-tumble and rotary diffusion producing viscoelastic behavior in unsteady flows. In order to understand how the nematodes tend to relax after the application of a stress, step strain 
tests were conducted. In this condition, at $t=0$ an instantaneous shear strain $\gamma=0.1$ was applied. The profile of stress relaxation is important to understand how the fluid behaves mechanically.

Figure 4.7: Relaxation function $G(t)$ of an active suspension of $\phi=1 \%$. The insert shows a zoom in the zone of transition after the active relaxation. The dashed line represents the active relaxation time $\tau=0.33 s$. [Source: the author.]

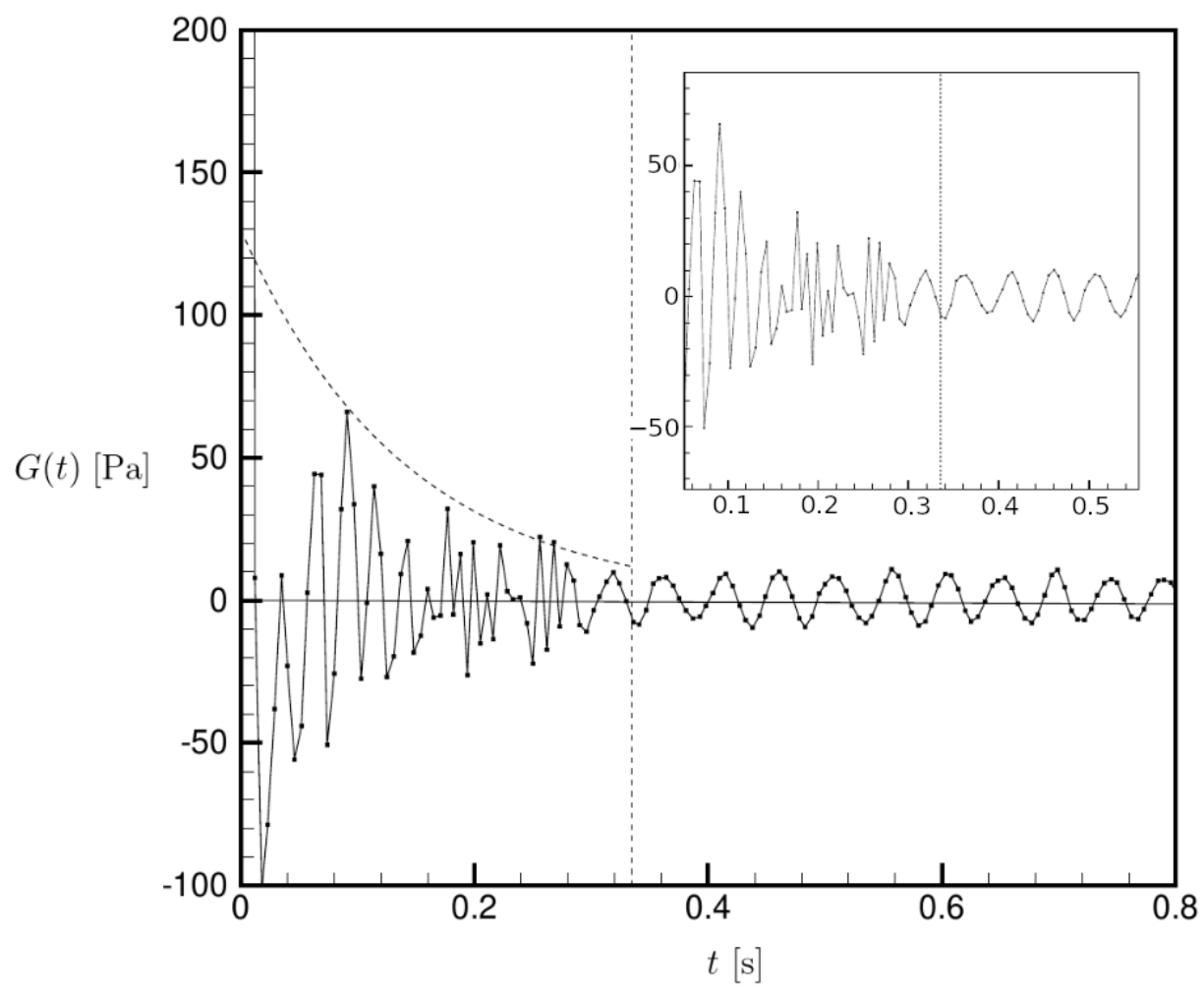

Upon start-up of rotation, an initial viscous stress jump occurs primarily due to the solvent viscosity. After this initial jump, particle orientations relax leading to a decrease in the measured viscosity as a result of the extensible stresslet. When rotation stops, the negative active stress persists for a certain duration, leading to a negative undershoot in the stress response, which corresponds to a retrograde torque. These mechanics lead to the oscillatory response shown in figure 4.7.

Considering the active particle as a slender body (COX AND MERZ, 1958), shown in figure 4.8 , in a prestressed weighted state (i.e. with an initial cuvature related to the intrinsic bending), the tangential forces will not resist exterior action, and will cause bias in the direction of application of the forces (NoviKov, 2006). Thus, the shear modulus might be negative. This is possible in inhomogeneous media. If the fluid constitutes a self-similar system of clusters of various sizes, 
each cluster will have its own resonance parameters and elastic characteristics, and will affect the effective shear modulus.

Figure 4.8: C. elegans as a slender body in a prestressed weighted state, i.e. curved. [Source: the author.]

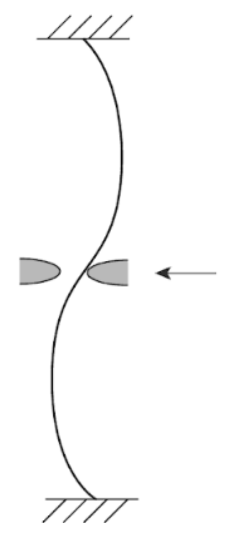

Source: (NoviKOV, 2006).

In fact, Bechtel and Khair (BECHTEL AND KHAIR, 2017) tested a modified Cox-Merz rule, in which the frequency-dependent linear viscosity approximately matches the steady shear viscosity at the maximum shear rate. The elastic component of viscosity is nonzero and peaks at intermediate frequencies, as could have been anticipated from the case of rigid rods. However, its sign can be negative in the case of pushers, which is at the origin of the stress undershoot after the cessation of rotation. Some constitutive models predicted pronounced oscillatory responses in elongational stress growth and stress relaxation after step shearing (ACIERNO et al., 1977).

The frequency of the oscillatory response is $f_{a}=20 \mathrm{~Hz}$. That means that during the active relaxation period $\tau_{a}$, the activity time is $\sim 1 / f_{a}=0.05 \mathrm{~s}$. Thus, the non-equilibrium condition is felt during the active relaxation time. If this frequency was smaller, the observed behavior would be very similar to that observed in viscoelastic passive suspensions due to the coupling between these time scales. In this case, the suspension would still have a non-instantaneous relaxation time, such as the one observed for the base suspension without nematodes (buffer), but without the oscillatory behavior, as shown in the dashed line in figure 4.7.

Figure 4.9 shows the active relaxation time $\tau$ as a function of the suspension volume fraction. As the volume fraction of the suspension increases, so does the active relaxation time. This is mainly due to the formation of aggregates and interactions between the particulate system, which 
increases the memory of the system.

Figure 4.9: Active relaxation time $\tau$ in seconds as a function of $\phi$. There is an increase in the time of relaxation due to the collective behavior previously observed. [Source: the author.]

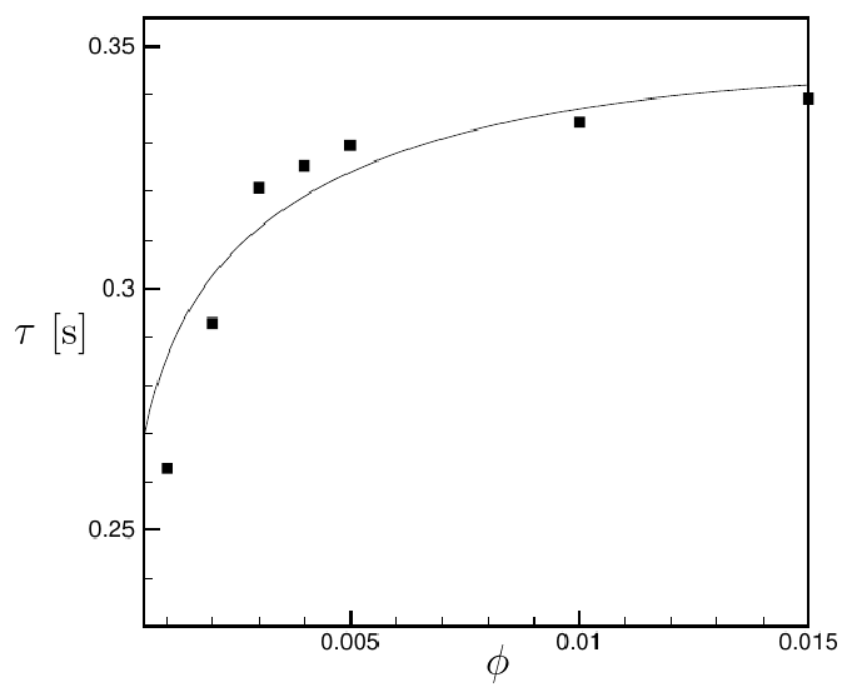

\subsubsection{Particle dipole stresslet - a physical interpretation}

According to Batchelor (BATCHELOR, 1977) the stress exerted by the surface $\boldsymbol{S}_{k}$ of the bounding sphere of radius $\ell$ on the fluid is given by

$$
\boldsymbol{S}_{k}=\oint_{S_{k}}\left[\boldsymbol{x}\left(\boldsymbol{n} \cdot \boldsymbol{\sigma}^{N}\right)-\mu(\boldsymbol{u n}+\boldsymbol{n u})\right]
$$

where $\boldsymbol{n}=\boldsymbol{x} / r$ is a unit vector, normal to the surface of the particle. In this case, without torque, the stresslet is the dipole produced by each particle. For particles prescribing a relative surface velocity (swimming gait), the second part of the integral does not depend on the swimming velocity. However, the first term involves the surface traction and without any other simplifications it can only be obtained by solving the flow everywhere. The bulk stress tensor of the suspension is given below:

$$
\boldsymbol{\sigma}=-\bar{p} \boldsymbol{I}+2 \mu \overline{\boldsymbol{D}}+n \overline{\boldsymbol{S}}
$$


The first two terms on the right-hand side of equation 4.9 are the contributions from the background continuous phase with an averaged pressure $\bar{p}$ and an average rate of strain $\bar{D}$. Here, $n$ is the average number of particles per unit volume and $\bar{S}$ is the average stresslet.

The active stress has the same tensorial form as the Brownian stress in suspensions of rod-like polymers. However, in active systems, the stress presented in equation 4.8 can be either positive or negative depending on the entropy production and fluctuations, which is directly related to the kind of microorganism: pusher or puller (S AINTILlan AND SHELlEY; SPAGNOLIE, 2011; 2015). As pointed by Kim and Karrila (KIM AND KARRILA, 1992), a second-rank-tensor called Stokeslet Green's function which relates velocity and force linearly can be obtained from the fundamental solution of creeping flow equation for the disturbance of velocity and pressure produced by a point force singularity. This tensor, given by $\boldsymbol{J}(\boldsymbol{r})=\left(\boldsymbol{I} / \boldsymbol{r}+\boldsymbol{r} \boldsymbol{r} / r^{3}\right)(1 / 8 \pi \mu)$, can be used with the traction force on the surface of an active particle to find the disturbance velocity. This solution can be expanded in several moments about the center of this microorganism: the monopole $\boldsymbol{F}$, the antisymmetric dipole $\boldsymbol{M}$ and the symmetric dipole $S$, or force, torque and stresslet, respectively. Therefore, considering a bounding sphere approximation around C. elegans, which permits a rigid particle description, we can use the multipole expansion. For these rigid particles free of body forces and torques we have:

$$
\boldsymbol{u}(\boldsymbol{x}) \sim \boldsymbol{S}:\left(1+\frac{\ell^{2}}{10} \nabla^{2}\right) \boldsymbol{J}
$$

where $S$ is the stresslet of a bounding spherical particle of radius $\ell$. Thus, for a spherical particle, $\boldsymbol{S}$ follows the third Faxén Law (RALLISON, 1978):

$$
\boldsymbol{S}=\frac{5}{2} V_{p} \eta\left(1+\frac{\ell^{2}}{10} \nabla^{2}\right) \boldsymbol{D}
$$

where $V_{p}=(4 / 3) \pi \ell^{3}$ is the particle volume, $\eta$ is the viscosity of the surrounding fluid, and $\boldsymbol{D}=\nabla \boldsymbol{u}+(\nabla \boldsymbol{u})^{T}$. If we denote the orientation direction of the active particle by $\boldsymbol{q}$, the stresslet component $\boldsymbol{q} \boldsymbol{q}$ is positive if the trust force is generated in front of the body and negative if it is generated behind, as shown in figure 4.10. Therefore, in the case of pushers, the stresslet strength is negative. For example, E. coli has a pronounced pusher behavior and all thrust comes from the 
rear part of its body.

Figure 4.10: Schematic of the stresslet exerted on the fluid for two types of cells, and the far-field velocity field relative to the swimming velocities of the cells. The arrows within the cells' bodies show the bodies' direction of "forward" locomotion and also the force by the cell bodies on the fluid. The external arrows show the associated forces by the flagella on the fluid. Plot (a) shows the thrust generated in front of the body (puller). Typical of the algae Chlamydomonas, the return stroke, with somewhat folded flagella, reverses the arrows. The cell body then moves backward. The return stroke results in less motion than the forward stroke, so the net motion over one complete cycle is forward. (b) Thrust generated behind the body (pusher). [Source: (ISHIKAWA, 2009).]

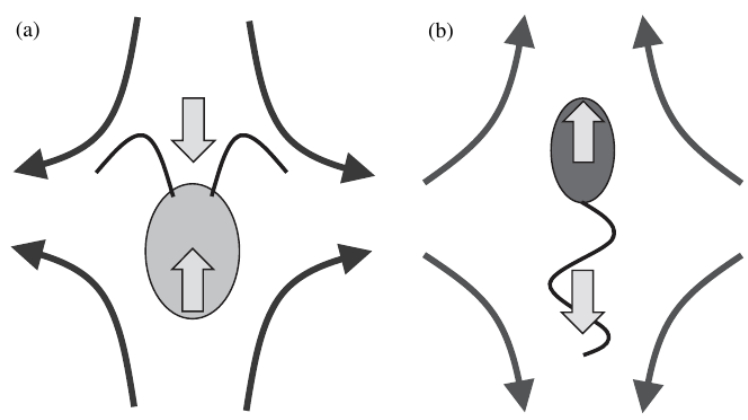

At low volume fractions, the interaction between the microorganisms is not considerable. Thus the active component becomes dominant and the effective viscosity decreases since the particle viscosity depends on the volume fraction, as seen in figure 4.6. As the volume fraction increases, the maximum packing becomes important due to the geometric characteristics of the microorganisms. This type of behavior was also observed by Sokolov and Aranson (SOKOLOV AND ARANSON, 2009a). Many different mechanisms contribute to this anomalous behavior (DOMBROWSKI et al., 2004).

By adding the extra term $\beta$ accounting for the permanent activity of microorganisms in equation 4.6, we are able to observe both puller and pusher behavior. In weak flows and at strong levels of activity, we obtained a negative particle viscosity. This result seems to show an nonphysical reduction in the viscous dissipation. However, since the particles are constantly injecting mechanical energy into the fluid, swimming cannot be considered a dissipative mechanism in nature.

Thus, the particle viscosity can be either positive or negative depending on the active power input of the particle. If it dominates viscous dissipation, there is a decrease in the suspension's 
viscosity. However, when viscous dissipation is the dominant contribution or the microorganism motion induces the same dissipative behavior as the applied stress, there is an increase in the effective viscosity.

\subsection{Chapter conclusions}

Motivated by the growing interest in active suspensions we conducted a series of experimental rheological analysis in a $C$. elegans suspension, a small nematode. It is important $t$ highlight that even the linear viscoelastic moduli of nematodes active suspensions are poorly understood and time-dependent rheological studies of nematodes' suspensions have been scarce in the current literature. With this study, we were able to observe both hydrodynamic and active mechanisms of the suspension during oscillatory shear and step strain tests.

Our experimental results have suggested a decrease in the fluids viscosity as the amount of nematodes increases until a certain critical volume fraction. We identified three important regions. The pure anisotropic shows an approximately linear increase of the viscosity is observed following the law of a rod body $\eta_{r} K \phi(\ell / a)^{2}$, where $\ell / a$ is the anisotropic aspect ratio. In the active elastic region the active particles insert elastic energy by their intrinsic motion on the bulk suspension. The suspension viscosity decreased as a consequence of this elastic energy production by the particles in the case of pusher microorganisms. After $\phi \sim 0.4 \%$ we have identified a region of structure formation, where collective viscous hydrodynamic particle-particle interactions that inhibit the elastic motion of the nematodes produce a nonlinear increasing of the suspension viscosity as the particle volume fraction increases.

Considering the similarities of the viscometric characteristics of the fluid in the low frequency regime, the first normal stress difference $\left(N_{1}\right)$ was also obtained. Unlike macromolecule suspensions, the active particle has a natural resistance to stretch, related to its intrinsic elasticity. Thus, there is anisotropy generated in the body of the nematode. However, as aggregates are formed, these structures tend to become anisotropic as they are stretching in the flow direction, causing an increase in the first normal stress difference.

In this chapter it is clear that the presence of microorganisms generates an increase in the complexity of the rheological characteristics of the suspension. Thus, for the correct representation 
in a constitutive model, simplifications are necessary. In the next chapter, a series of simplifications will be applied to a numerical model, seeking to obtain validation of the resulting behavior. 


\section{Filament Kinematic Simulation}

In the previous chapters, we obtained results of the kinematic behavior of one nematode and rheological characterization of the active suspension. The complexity of the microorganism and its viscoelastic membrane makes it difficult to define a constitutive model. For this reason, some simplifications are needed. The results of the stress relaxation function obtained experimentally suggest an orientational behavior. Thus, we believe that the nematode can be simplified by a flexible orientational filament.

In this chapter, we intend to simulate the motion of a slender filament, basically an idealized model of anguilliform swimming. In order to propose a rheological model for the suspension of nematodes, it is important to simplify the model. In this sense, we intende to study how the kinematics of a flexible filament can be compared to that of a nematode. The results presented here, using the algorithm IB2d proposed by Battista (BATTISTA et al., 2018), are extremely important in defining the basis of the orientational filaments that are used in the next chapter.

In the 1970s, Peskin (WHITE et al., 2002) developed the immersed-boundary method (IBM) to simulate flexible membranes in fluid flows. The membrane-fluid interaction is accomplished by distributing membrane forces as local fluid forces and updating the membrane configuration according to the local flow velocity. Since then, the IBM has been widely employed to study various situations, including cell deformation in micropipettes, leukocyte adhesion and movement, multiphase flows, red blood cell deformation and aggregation in shear flows and the behavior of biofilms (BATTISTA et al., 2017). One distinguishing feature of this approach is the ability to perform the entire simulation on a fixed Cartesian grid (BATTISTA et al., 2015). Thus, the IBM is an elegant way to fully couple the motion of a fluid and deformations of an immersed elastic structure.

Controlling the choice of behavioral output is a central function of the nervous system (GoSH AND EMMONS, 2008). Study of the regulation of behavior in the nematode C. elegans takes advantage of the fact that its behavior is relatively simple and its nervous system contains a constant number of neurons whose pattern of synaptic connectivity is known (CHEN et al.; WHITE et al., 2006; 1986). Depending on the availability of food and health, nematodes can move symmetrically or asymmetrically, as will be discussed in this chapter. 


\subsection{Immersed boundary method}

The conservation of momentum equations that govern an incompressible and viscous fluid are written as the following set of coupled partial differential equations namely conservation of momentum and mass

$$
\begin{gathered}
\rho\left(\frac{\partial \boldsymbol{U}}{\partial t}(x, t)+\boldsymbol{U}(x, t) \cdot \nabla \boldsymbol{U}(x, t)\right)=-\nabla p(x, t)+\mu \Delta \boldsymbol{U}(x, t)+\boldsymbol{F}(x, t), \\
\nabla \cdot \boldsymbol{U}(x, t)=0,
\end{gathered}
$$

where $\boldsymbol{U}(x, t)$ is the fluid velocity, $p(x, t)$ is the pressure, $\boldsymbol{F}(x, t)$ is the force per unit area applied to the fluid by the immersed boundary, $\rho$ and $\mu$ are the fluid's density and dynamic viscosity, respectively. The independent variables are the time $t$ and the position $x$. The variables $\boldsymbol{U}, p$ and $\boldsymbol{F}$ are all written in an Eulerian frame on the fixed Cartesian mesh $x$. The Navier-Stokes equations in Eulerian domain, e.g., updated $\boldsymbol{U}^{n+1}$ and $p^{n+1}$ from $\boldsymbol{U}^{n}$ and $\boldsymbol{F}^{n}$ are solved using the fast Fourier Transform and more details can be found in the appendix A. (COOLEY AND TUKEY, 1965).

The equations that couple the motion of the fluid to deformations of the structure are written as integral equations. These interaction equations handle all communication between the fluid (Eulerian) grid and immersed boundary (Lagrangian grid). They are given as the following integral equations with delta function kernels

$$
\begin{gathered}
\boldsymbol{F}(x, t)=\int \boldsymbol{f}(s, t) \delta(\boldsymbol{x}-\boldsymbol{X}(s, t)) d q \\
\boldsymbol{u}(\boldsymbol{X}(x, t))=\int \boldsymbol{U}(s, t) \delta(\boldsymbol{x}-\boldsymbol{X}(s, t)) d \boldsymbol{x},
\end{gathered}
$$

where $\boldsymbol{f}(s, t)$ is the force per unit area imposed by elastic deformations in the immersed structure onto the fluid as a function of Lagrangian position, $s$, and time, $t, \delta(x)$ is a three-dimensional delta 
function, and $\boldsymbol{X}(s, t)$ gives the Cartesian coordinates at time $t$ of the material point labeled by the Lagrangian parameter, $s$. The velocity of the fluid, $\boldsymbol{u}(\boldsymbol{X}(x, t))$, is given by the derivative of the Cartesian coordinates $\boldsymbol{X}(s, t)$.

Equation 5.3 applies the force from the immersed boundary to the fluid through the external forcing term in equation 5.1. After that, equation 5.4 moves the boundary at the local fluid velocity. This enforces the no-slip condition. Each integral transformation uses a three dimensional Dirac delta function kernel, $\delta$, to convert Lagrangian variables to Eulerian variables and vice versa. To either hold the geometry nearly rigid or prescribe the motion of the immersed structure, all of the Lagrangian points along the immersed boundary are tethered to target points. We do this through a penalty force formulation of $\boldsymbol{f}(s, t)$, written as the following

$$
\boldsymbol{f}(s, t)=k_{t}(\boldsymbol{Y}(s, t)-\boldsymbol{X}(s, t)),
$$

where $k_{t}$ is a stiffness coefficient and $\boldsymbol{Y}(s, t)$ is the prescribed position of the target boundary. Note that $\boldsymbol{Y}(s, t)$ is a function of both the Lagrangian parameter, $s$, and time $t$ and in these models $k_{t}$ was chosen to be large so that it would effectively drag the Lagrangian points into the prescribed positions. The swimmer is modelled as a chain of springs and beams. Springs allow for stretching and compressing of the successive Lagrangian points, while beams allow for bending. Their force equations can be written as the following,

$$
\begin{gathered}
F_{s p r}=-k_{s p r}\left(1-\frac{R_{L}}{\left\|\boldsymbol{X}_{S}-\boldsymbol{X}_{M}\right\|}\right) \cdot\left(\boldsymbol{X}_{S}-\boldsymbol{X}_{M}\right)+b_{s} \frac{d}{d t}\left\|\boldsymbol{X}_{S}-\boldsymbol{X}_{M}\right\|, \\
F_{\text {beam }}=-k_{\text {beam }} \frac{\partial^{4}}{\partial s^{4}}\left(\boldsymbol{X}(s, t)-\boldsymbol{X}_{B}(s, t)\right),
\end{gathered}
$$

where $k_{s p r}$ and $k_{\text {beam }}$ are the spring stiffness and beam stiffness coefficients, respectively, and $b_{s}$ is the damping coefficient. For the linear spring forces, the terms $\boldsymbol{X}_{M}$ and $\boldsymbol{X}_{S}$ represent the positions in Cartesian coordinates of the master and slave Lagrangian nodes at time $t$ and $R_{L}$ is the spring's corresponding resting length. For the bending force, $\boldsymbol{X}_{B}(s, t)$ represents the curvature of the configuration at time, $t$. In other words, considering the nematode's model, the resistance to stretching 
between successive Lagrangian points is achieved by modeling the connections with Hookean (or non-Hookean) springs, while the resistance to bending between three successive Lagrangian point is modeled by using a noninvariant beam, as shown in figures 5.1 and 5.2, respectively. Thus, the curvature is changed by interpolating through two different configurative phases of the swimmer.

Figure 5.1: Illustrating the idea behind the damped springs model with a damped spring at equilibrium (left) and a damped spring stretched a distanced beyond its resting length $R_{L}$ (right). Resistance to stretching or bending is opposed by a friction-like term that is proportional to the velocity of deformation. [Source: the author.]

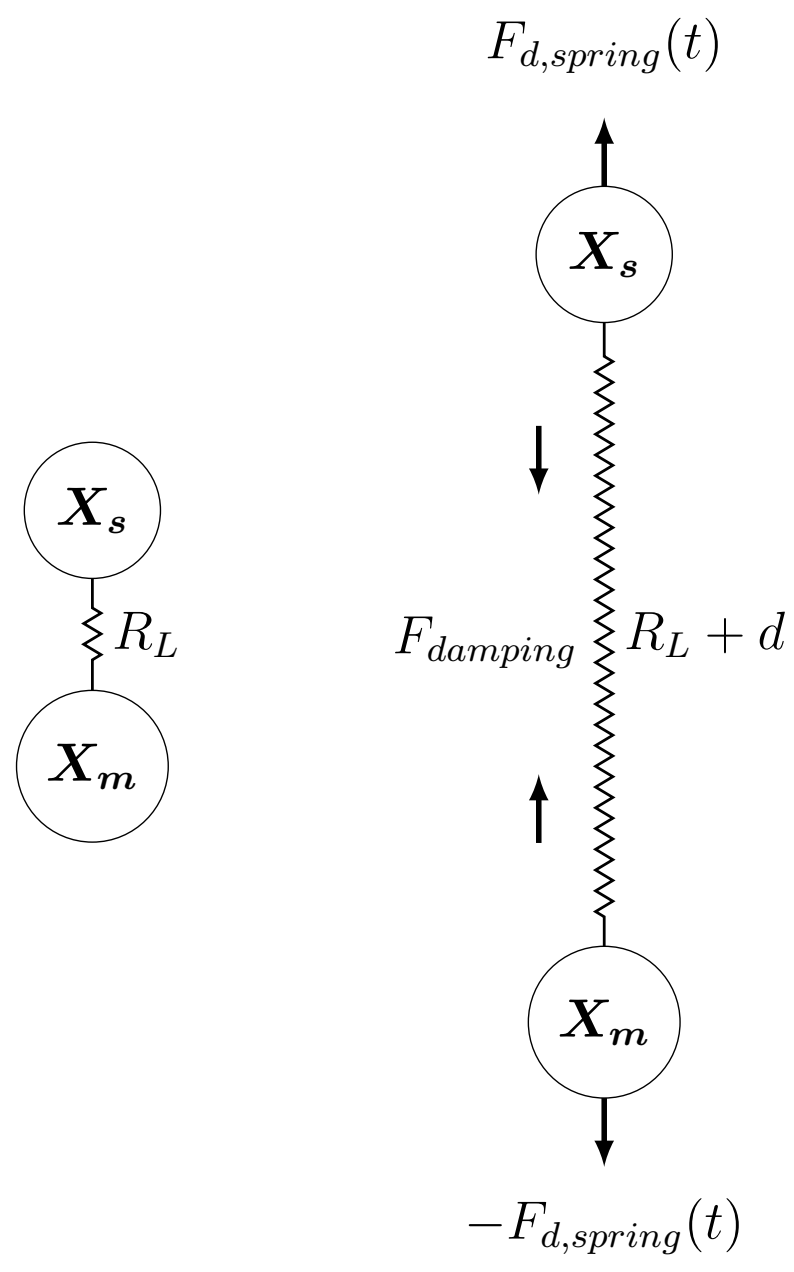


Figure 5.2: Illustrating the idea behind the noninvariant beam model. The beam has a preferred x-and y-curvature, given by $C_{x}$ and $C_{y}$, respectively. When the configuration is deformed so curvatures are now $C_{x}^{\prime}$ and $C_{y}^{\prime}$ respectively, a restoring force drives the configuration towards its preferred equilibrium position. [Source: the author.]
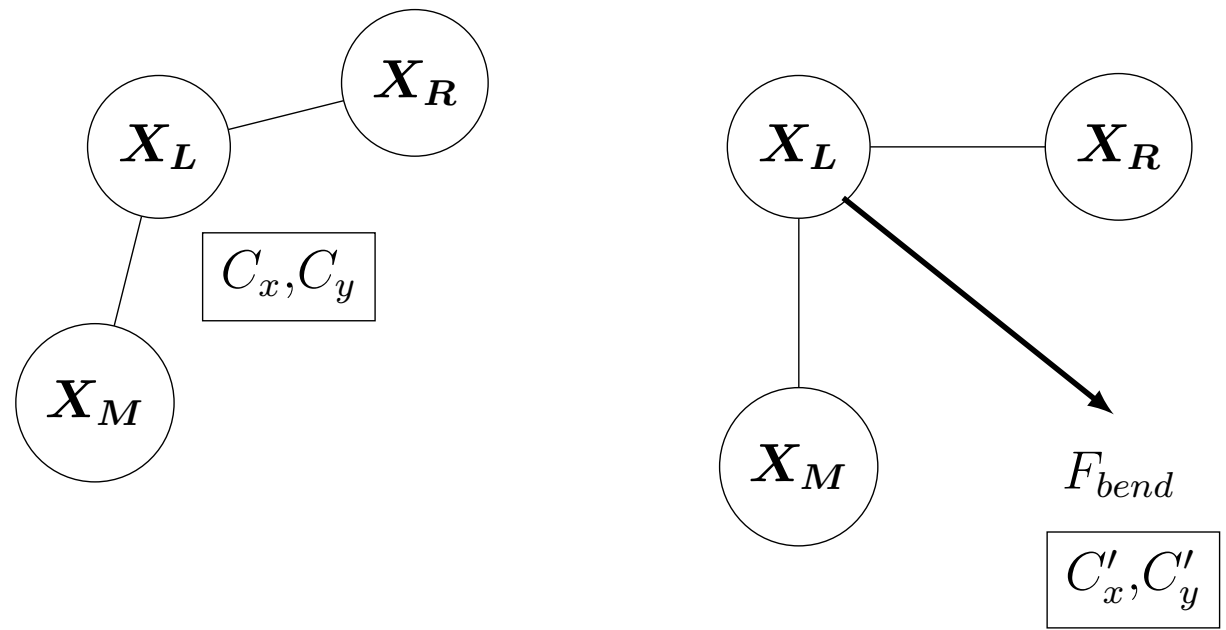
Basically, the numerical algorithm is:

$\circ$ Step 1: Find the force $f^{n}$ on the immersed boundary, from the current boundary configuration, $\boldsymbol{X}^{n}$ using equation 5.5.

- Step 2: Use equation 5.3 to spread the boundary force from the Lagrangian bondary mesh to the Eulerian fluid lattice points.

- Step 3: Solve the Navier-Stokes equations (equations 5.1 and 5.2) on the Eulerian grid. In this condition, $\boldsymbol{U}^{n+1}$ and $p^{n+1}$ are obtained updating $\boldsymbol{U}^{n}, p^{n}, \boldsymbol{F}^{n}$ based on FFT (more details are shown in the appendix B).

○ Step 4: Uptade the material positions, $\boldsymbol{X}^{n+1}$, using the local fuid velocities, $\boldsymbol{U}^{n+1}$, computed from $\boldsymbol{U}^{n+1}$ and equation 5.4, e.g. move the immersed structure at the local fluid velocities thereby enforcing no-slip boundary conditions.

\subsection{Swimmer modelling}

During crawling locomotion, $C$. elegans obtains its maximal muscle activity and maximal body curvature. These show a small but important phase-shift, with maximal muscle activity preceding maximal body curvature. Phase-shifts are comparable between dorsal and ventral muscle but the magnitude increases along the body of the worm and is significantly higher in posterior muscle compared to anterior and mid-body muscle (VICTORIA J. BUTLER et al., 2015). Such a relationship has also been observed for the longitudinal red muscle fibres of many fish species that display anguilliform locomotion similar to $C$. elegans. An idealized swimmer was constructed by taking a line segment and attaching a polynomial section to it, as shown in figure 5.4. The straight portion composes $28 \%$ of the total length of the body, while the polynomial, i.e., $y=x^{3}$, portion makes up the remaining $72 \%$. The polynomial portion was found starting from $x=0$ and adding equally spaced points (at a distance twice of that of the fluid mesh).

In terms of the Eulerian mesh, it is necessary to perform a mesh convergence analysis. However, we do not have the exact analytical solution. Thus, meshes with different nodes were used and the position of the filament head $X_{e}$ after 4 strokes, as shown in figure 5.3. It is important to observe that increasing the resolution, while decreasing the error, results in significant increases in 
the computational time required to run a simulation. In general this computational expense scaling canbe written as

$$
\text { computational time }=(\text { resolution factor })^{\text {dimension }}
$$

Figure 5.3: Swimming strokes performed by the head, $X_{e}$, based on the number of the mesh's nodes $n$. The higher resolution cases demonstrate better forward swimming performance [Source: the author.]

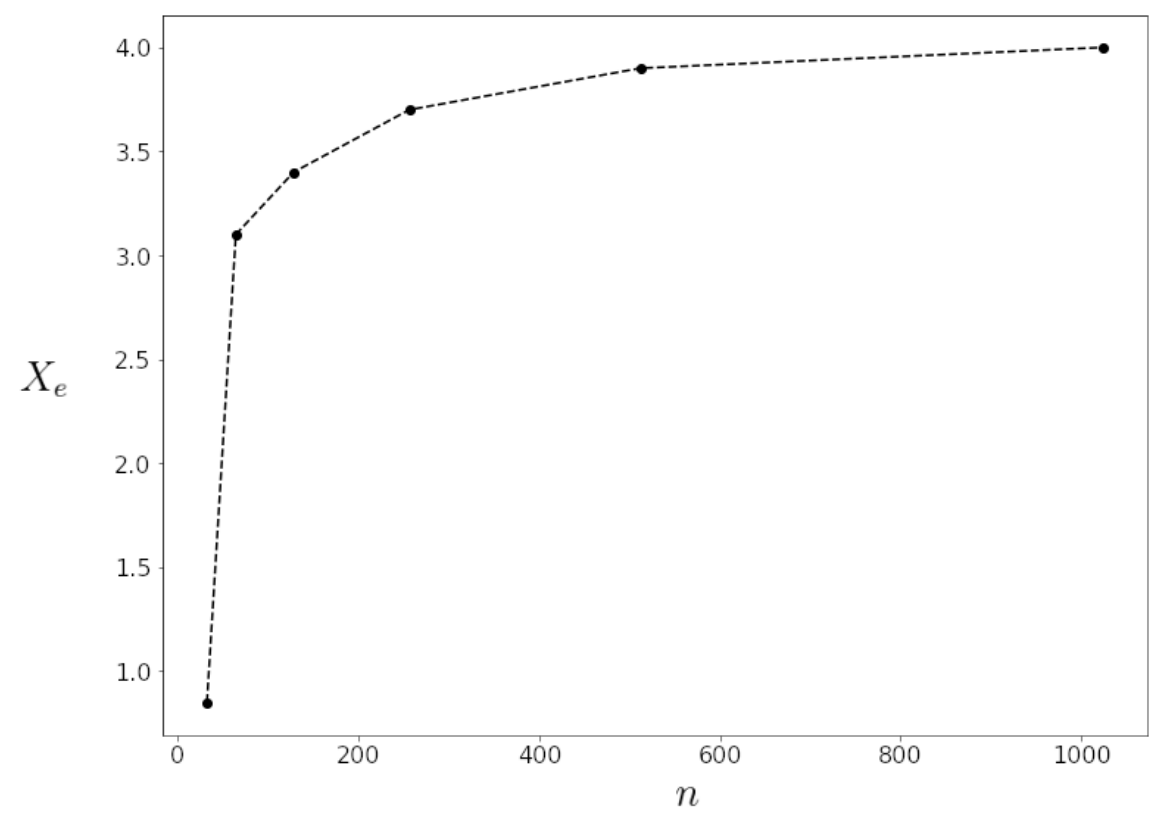

One must inquire how much accuracy is required for a problem, e.g., validating that the swimmer is capturing biologically relevant kinematics and/or swimming speeds at certain resolutions. In our case, we observed that using $\left(n_{x}, n_{y}\right)=512 \times 256$ resolution is enough.

We will model the swimmer's body switching between two curvature states, phase 1 and phase 2, shown in figure 5.4. Each phase was defined by negating the y-coordinate of the polynomial portion of the body. This is a biologically relevant modeling assumption because muscle activation patterns produce specific intrinsic curvatures for a swimmer's body. When the Lagrangian points interact with the surrounding fluid, locomotion emerges. The curvatures to be interpolated between were computed as follows: 
Figure 5.4: The curvature of the nematode is produced based on a straight line, shown by the white arrow and a interpolation in a polynomial section. The plot on top shows the vorticity field at $t=90[\mathrm{~s}]$ for curvature at phase 1 , while the bottom plot shows the vorticity field at $t=100[\mathrm{~s}]$ for curvature at phase 2. The idealized anguilliform swimmer moves forward due to vortices being shed off its caudal end during each stroke. [Source: the author.]
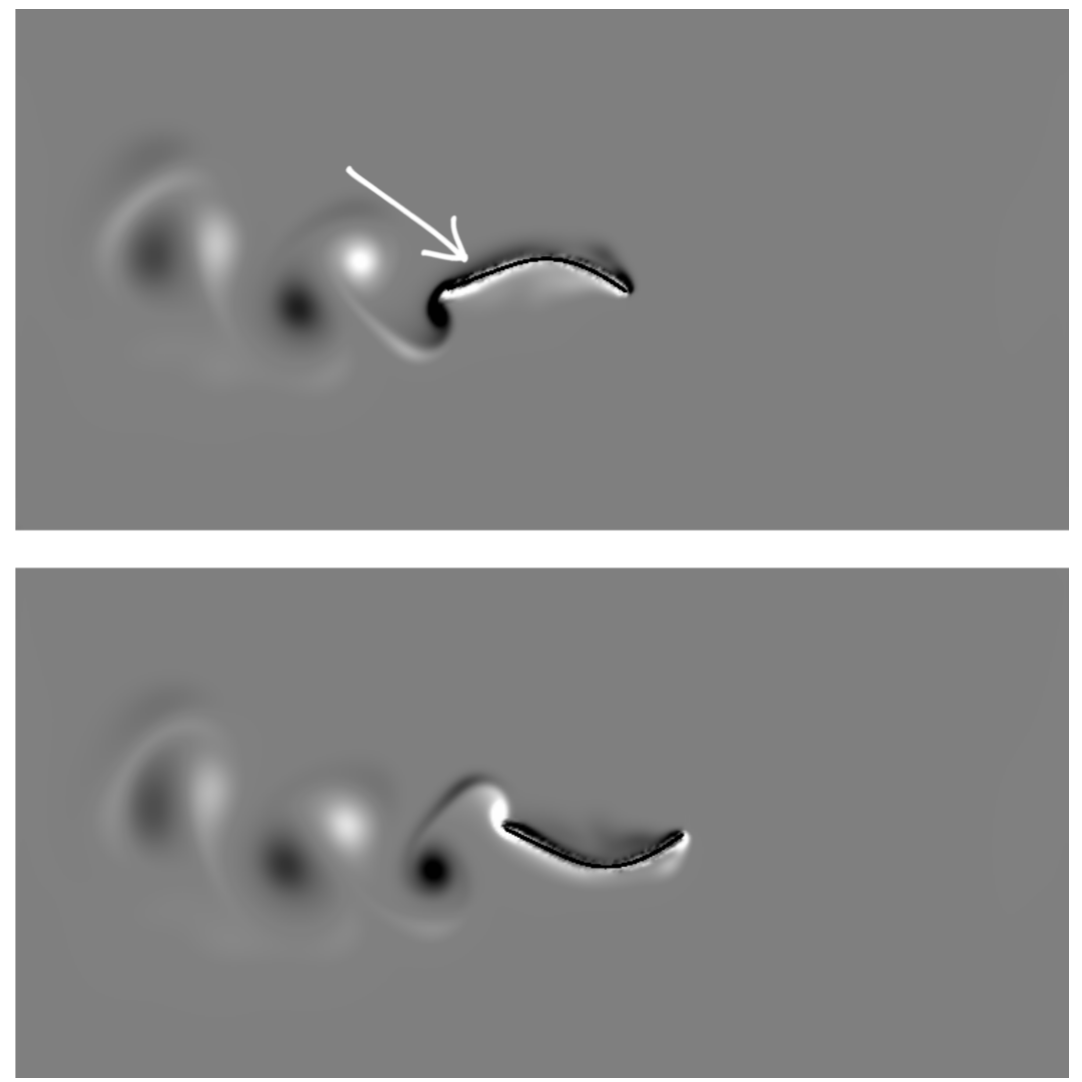

$$
\begin{gathered}
C_{x}^{\delta}=x_{\text {Lag }}^{\delta}(s)-2 x_{\text {Lag }}^{\delta}(s+1)+x_{\text {Lag }}^{\delta}(s+2) \\
C_{y}^{\delta}=y_{\text {Lag }}^{\delta}(s)-2 y_{\text {Lag }}^{\delta}(s+1)+y_{\text {Lag }}^{\delta}(s+2)
\end{gathered}
$$

where $s$ runs over all interior points along the swimmer's body and $\delta$ refers to phase 1 or 2 . Basically, instead of changing the explicit positions of the Lagrangian points, we update the curvatures $C_{x}^{\delta}$ and $C_{y}^{\delta}$. We also define the downstroke and upstroke to be moving between phase 1 to phase 2 and phase 2 to phase 1, respectively. Furthermore we also define 1 strokeperiod to encompass both the upstroke and downstroke. 
To solve the position, velocity and acceleration of each point, we use interpolation. Considering two points $\boldsymbol{a}$ and $\boldsymbol{b}$, we can parameterize a straight line between them in the following way:

$$
[x(t), y(t)]=\boldsymbol{g}_{0}(t)=\boldsymbol{a}+\frac{t}{t_{1}}(\boldsymbol{b}-\boldsymbol{a}),
$$

for $t \in\left[0, t_{1}\right]$, we have $\boldsymbol{g}_{0}(0)=\boldsymbol{a}$ and $\boldsymbol{g}_{1}\left(t_{1}\right)=\boldsymbol{b}$. We can interpolate point $\boldsymbol{b}$ with another point $\boldsymbol{c}$

$$
[x(t), y(t)]=\boldsymbol{g}_{1}(t)=\boldsymbol{b}+\frac{t-t_{1}}{t_{2}-t_{1}}(\boldsymbol{c}-\boldsymbol{b}),
$$

for $t \in\left[t_{1}, t_{2}\right]$. The piecewise linear interpolant between all three points could be written as

$$
[x(t), y(t)]=\left(\begin{array}{l}
\boldsymbol{g}_{0}(t) \\
\boldsymbol{g}_{1}(t)
\end{array}\right)= \begin{cases}\boldsymbol{a}+\frac{t}{t_{1}}(\boldsymbol{b}-\boldsymbol{a}), & 0 \leq t \leq t_{1} \\
\boldsymbol{b}+\frac{t-t_{1}}{t_{2}}(\boldsymbol{c}-\boldsymbol{b}), & 0 t_{1} \leq t \leq t_{2}\end{cases}
$$

This can be extended to a larger collection of points. If points $\boldsymbol{a}, \boldsymbol{b}$ and $\boldsymbol{c}$ are matrices, where each column contains $N-(x, y)$ points:

$$
\boldsymbol{A}=\left[\begin{array}{cc}
x_{0}^{a} & y_{0}^{a} \\
x_{1}^{a} & y_{1}^{a} \\
\vdots & \vdots \\
x_{N}^{a} & y_{N}^{a}
\end{array}\right], \boldsymbol{B}=\left[\begin{array}{cc}
x_{0}^{b} & y_{0}^{b} \\
x_{1}^{b} & y_{1}^{b} \\
\vdots & \vdots \\
x_{N}^{b} & y_{N}^{b}
\end{array}\right] \text {, and } \boldsymbol{C}=\left[\begin{array}{cc}
x_{0}^{c} & y_{0}^{c} \\
x_{1}^{c} & y_{1}^{c} \\
\vdots & \vdots \\
x_{N}^{c} & y_{N}^{c}
\end{array}\right]
$$

we can write an analogous spline interpolant as follows

$$
[x(t), y(t)]=\left(\begin{array}{l}
\boldsymbol{G}_{1}(t) \\
\boldsymbol{G}_{1}(t)
\end{array}\right)== \begin{cases}\boldsymbol{A}+\frac{t}{t_{1}}(\boldsymbol{B}-\boldsymbol{A}), & 0 \leq t \leq t_{1} \\
\boldsymbol{B}+\frac{t-t_{1}}{t_{2}}(\boldsymbol{C}-\boldsymbol{B}), & t_{1} \leq t \leq t_{2}\end{cases}
$$


Moreover, rather than use linear interpolation, which leads to instantaneous accelerations, it's possible to use a cubic polynomial between successive points. So the intepolant could be written as

$$
g(t)=\boldsymbol{a}+h(t)(\boldsymbol{b}-\boldsymbol{a})
$$

where $h(t)$ is a cubic polynomial, e.g.,

$$
h(t)=d_{0}+d_{1} t+d_{2} t^{2}+d_{3} t^{3} .
$$

It leads to the following conditions

$$
\begin{aligned}
& \text { continuity }\left\{\begin{array}{l}
g(0)=0 \\
g(1)=1
\end{array}\right. \\
& \text { continuous velocities }\left\{\begin{array}{l}
g^{\prime}(0)=0 \\
g^{\prime}(1)=0
\end{array}\right. \\
& \text { no instantaneous accelerations }\left\{\begin{array}{l}
g^{\prime \prime}(0)=0 \\
g^{\prime \prime}(1)=0
\end{array}\right.
\end{aligned}
$$

It is possible to solve this over-constrained system interpolating mediary points $p_{1}$ and $p_{2}$, such that we partition the interval $[0,1]$ into three regions $0<p_{1}<p_{2}<1$. Therefore we can consider the following interpolant:

$$
g(t)= \begin{cases}g_{0}(t)=a_{0}+a_{1} t+a_{2} t^{2}+a_{3} t^{3} & 0 \leq t \leq p_{1} \\ g_{1}(t)=b_{0}+b_{1} t+b_{2} t^{2}+b_{3} t^{3} & p_{1} \leq t \leq p_{2} \\ g_{2}(t)=c_{0}+c_{1} t+c_{2} t^{2}+c_{3} t^{3} & p_{2} \leq t \leq 1\end{cases}
$$


which leads to

$$
\begin{aligned}
& \text { continuity }\left\{\begin{array}{l}
g(0)=0 \\
g(1)=1 \\
g_{0}\left(p_{1}\right)=g_{1}\left(p_{1}\right) \\
g_{1}\left(p_{2}\right)=g_{2}\left(p_{2}\right)
\end{array}\right. \\
& \text { continuous velocities }\left\{\begin{array}{l}
g^{\prime}(0)=0 \\
g^{\prime}(1)=0 \\
g_{0}^{\prime}\left(p_{1}\right)=g_{1}^{\prime}\left(p_{1}\right) \\
g_{1}^{\prime}\left(p_{2}\right)=g_{2}^{\prime}\left(p_{2}\right)
\end{array}\right. \\
& \text { no instantaneous accelerations }\left\{\begin{array}{l}
g^{\prime \prime}(0)=0 \\
g^{\prime \prime}(1)=0 \\
g_{0}^{\prime \prime}\left(p_{1}\right)=g_{1}^{\prime \prime}\left(p_{1}\right) \\
g_{1}^{\prime \prime}\left(p_{2}\right)=g_{2}^{\prime \prime}\left(p_{2}\right)
\end{array}\right.
\end{aligned}
$$

This gives the following linear system to solve, with variables $p_{1}$ and $p_{2}$ 


$$
\left[\begin{array}{cccccccccccc}
1 & 0 & 0 & 0 & 0 & 0 & 0 & 0 & 0 & 0 & 0 & 0 \\
0 & 1 & 0 & 0 & 0 & 0 & 0 & 0 & 0 & 0 & 0 & 0 \\
0 & 0 & 1 & 0 & 0 & 0 & 0 & 0 & 0 & 0 & 0 & 0 \\
1 & p_{1} & p_{1}^{2} & p_{1}^{3} & -1 & -p_{1} & -p_{1}^{2} & -p_{1}^{3} & 0 & 0 & 0 & 0 \\
0 & 1 & 2 p_{1} & 3 p_{1}^{2} & 0 & -1 & -2 p_{1} & -3 p_{1}^{2} & 0 & 0 & 0 & 0 \\
0 & 0 & 2 & 6 p_{1} & 0 & 0 & -2 & -6 p_{1} & 0 & 0 & 0 & 0 \\
0 & 0 & 0 & 0 & 1 & p_{2} & p_{2}^{2} & p_{2}^{3} & -1 & -p_{2} & -p_{2}^{2} & -p_{2}^{3} \\
0 & 0 & 0 & 0 & 0 & 1 & 2 p_{2} & 3 p_{2}^{2} & 0 & -1 & -2 p_{2} & -3 p_{2}^{2} \\
0 & 0 & 0 & 0 & 0 & 0 & 2 & 6 p_{2} & 0 & 0 & -2 & -6 p_{2} \\
0 & 0 & 0 & 0 & 0 & 0 & 0 & 0 & 1 & 1 & 1 & 1 \\
0 & 0 & 0 & 0 & 0 & 0 & 0 & 0 & 0 & 1 & 2 & 3 \\
0 & 0 & 0 & 0 & 0 & 0 & 0 & 0 & 0 & 0 & 2 & 6
\end{array}\right]\left(\begin{array}{l}
a_{0} \\
a_{1} \\
a_{2} \\
a_{3} \\
b_{0} \\
b_{1} \\
b_{2} \\
b_{3} \\
c_{0} \\
c_{1} \\
c_{2} \\
c_{3}
\end{array}\right)=\left(\begin{array}{l}
0 \\
0 \\
0 \\
0 \\
0 \\
0 \\
0 \\
0 \\
0 \\
1 \\
0 \\
0
\end{array}\right)
$$

As $p_{1} \rightarrow 0$ (or $p_{2} \rightarrow 1$ ), we see that the initial acceleration (or final deceleration) becomes greater and greater in magnitude. In practice, these parameters match the acceleration to the kinematics coming from a biological system or engineering system. We will vary these points symmetrically about the interpolation interval and consider the following cases:

$$
\begin{aligned}
& \left(p_{1}, p_{2}\right)=(0.1,0.9) \\
& \left(p_{1}, p_{2}\right)=(0.2,0.8) \\
& \left(p_{1}, p_{2}\right)=(0.3,0.7) \\
& \left(p_{1}, p_{2}\right)=(0.4,0.6)
\end{aligned}
$$

Upon varying these points, we need to make sure that our interpolation function is consistent, that is, we need to solve the linear system to get the proper coefficients for the spline interpolant. Based on that, we are able to compute the polynomial part of the nematode model. The terms obtained fo each condition tested in these chapter can be found on the appendix C. 


\subsection{Results and discussions}

\subsubsection{Symmetrical changes}

Considering the symmetrical change of the mediary points $\left(p_{1}, p_{2}\right)$ as shown in equation 5.24 , we can compute the coefficients of the interpolation function. Figure 5.5 shows the body curvature of the nematode depending on $\left(p_{1}, p_{2}\right)$. We observe that when $\left(p_{1}, p_{2}\right)=(0.1,0.9)$, the nematode has higher curvature. That means that $C$. elegans transform more elastic energy into propulsion in this case.

Figure 5.5: Nematode curvature from $t=0$ time steps to $t=90$ time steps. The black nematode represents $\left(p_{1}, p_{2}\right)=(0.1,0.9)$, the red nematode $\left(p_{1}, p_{2}\right)=(0.2,0.8)$, the blue $\left(p_{1}, p_{2}\right)=$ $(0.3,0.7)$ and the green $\left(p_{1}, p_{2}\right)=(0.4,0.6)$. [Source: the author.]

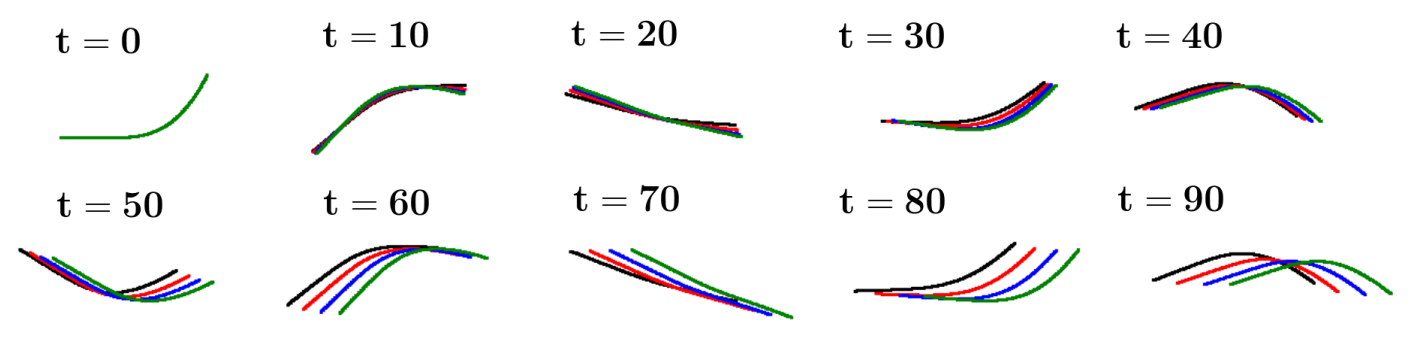

It is possible to observe in figure 5.6 an 5.7 that the swimming performance is changed. Higher magnitudes of velocity and acceleration, as presented when $\left(p_{1}, p_{2}\right)=(0.4,0.6)$ lead to faster swimming motion. Moreover, when the swimming velocity is higher, the nematode tends to move faster toward the end of the channel, as depicted in figure 5.8. Figure 5.9 shows the trajectory of the nematode depending on the interpolation points. It is possible to see the nonlinearity caused by the difference in symmetry. 
Figure 5.6: Forward distance swam in bodylenghts vs. swimming strokes performed in the case of symmetric interpolation points $\left(p_{1}, p_{2}\right)$. The dashed line represents $\left(p_{1}, p_{2}\right)=(0.4,0.6)$, the dotted line $\left(p_{1}, p_{2}\right)=(0.3,0.7)$, the dash-dot line $\left(p_{1}, p_{2}\right)=(0.2,0.8)$ and the solid line $\left(p_{1}, p_{2}\right)=$ $(0.1,0.9)$. [Source: the author.]

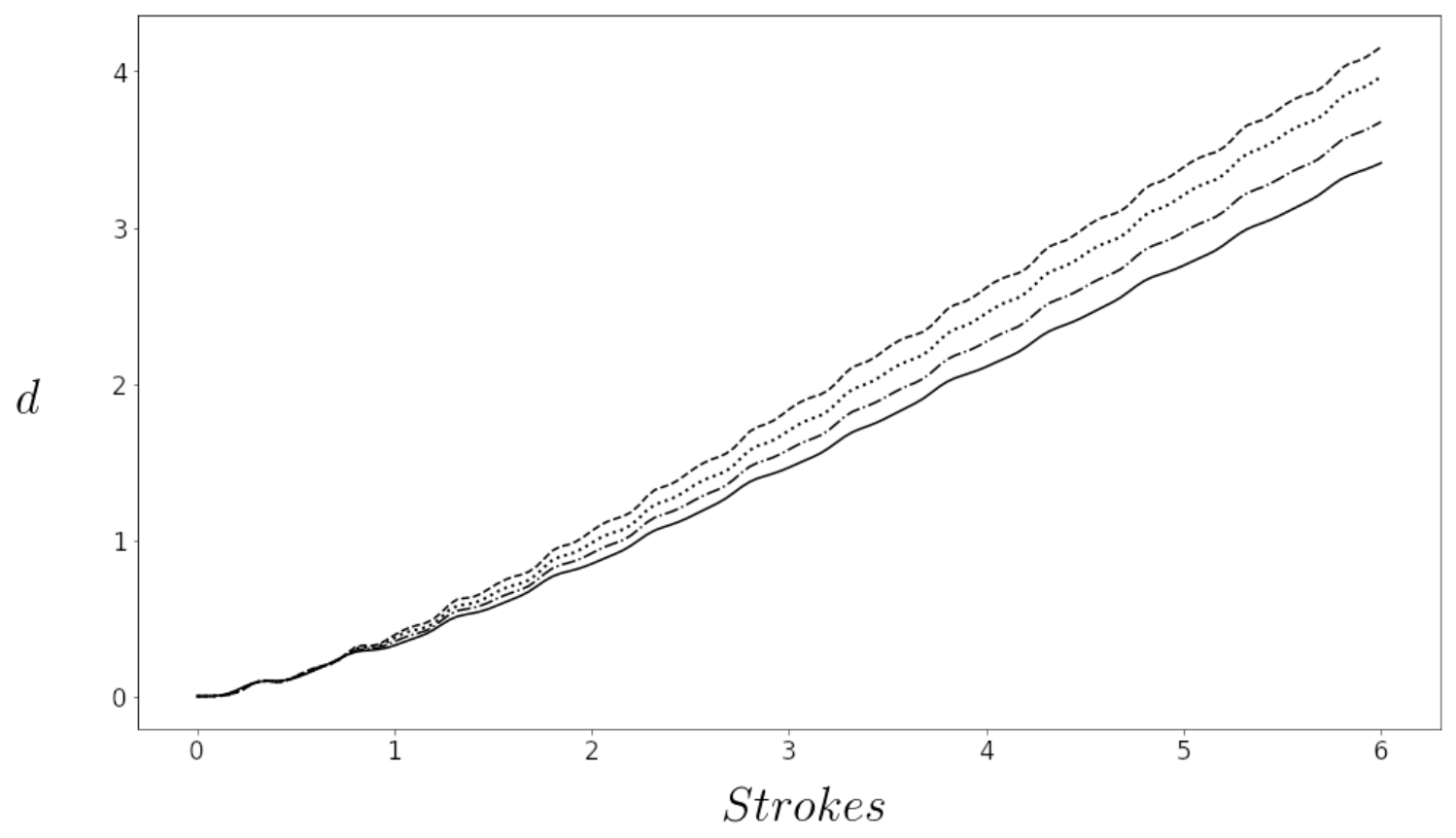


Figure 5.7: Forward velocity in bodylenghts per strokes vs. swimming strokes obtained in the cases of symmetric interpolation points $\left(p_{1}, p_{2}\right)$. The first plot represents $\left(p_{1}, p_{2}\right)=(0.1,0.9)$, the second plot $\left(p_{1}, p_{2}\right)=(0.2,0.8)$, the third $\left(p_{1}, p_{2}\right)=(0.3,0.7)$ and the last plot $\left(p_{1}, p_{2}\right)=(0.4,0.6)$. [Source: the author.]

$v$
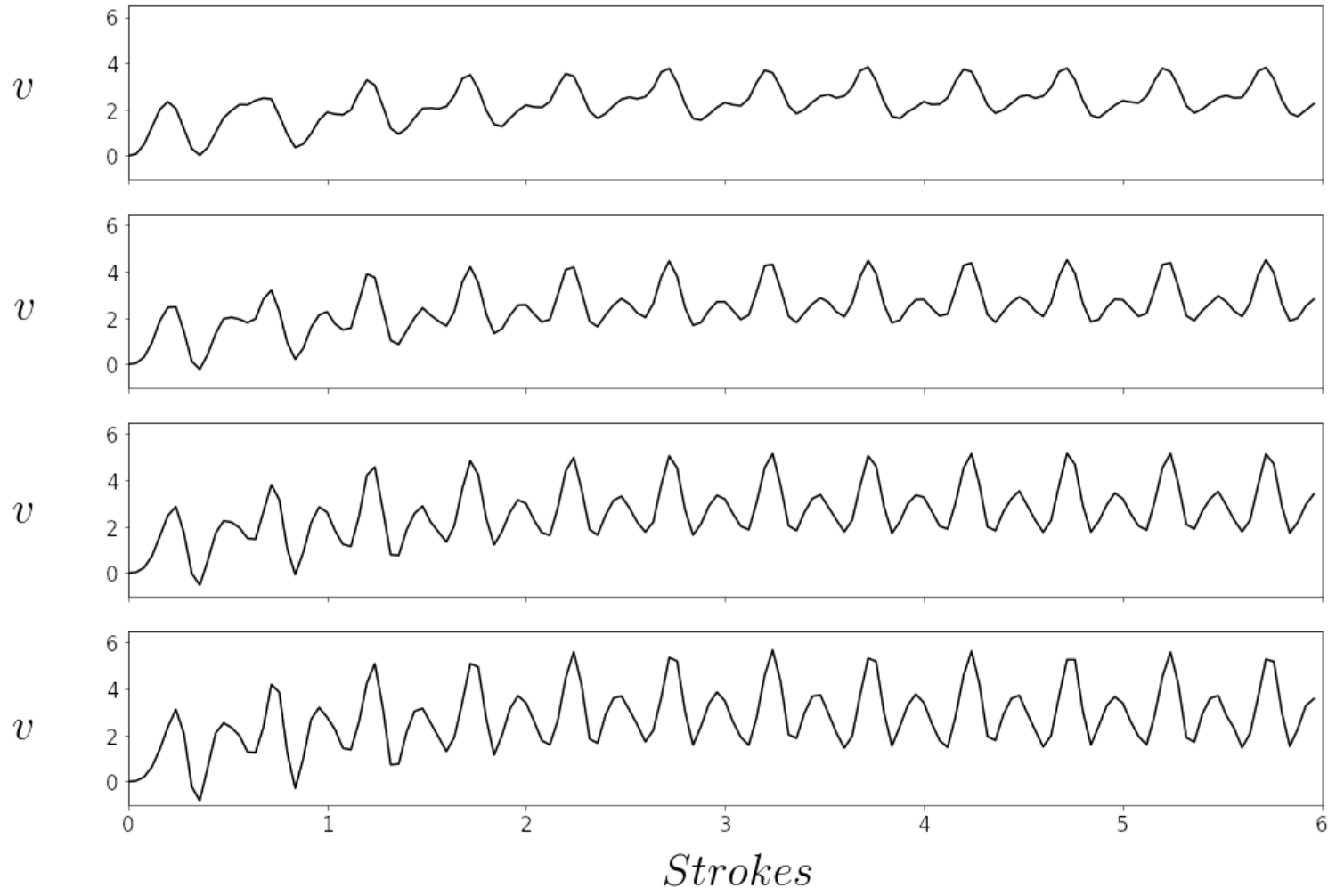
Figure 5.8: Zoom of the forward velocity in bodylenghts per strokes vs. swimming strokes performed in the case of symmetric interpolation. The dashed line represents $\left(p_{1}, p_{2}\right)=(0.4,0.6)$, the dotted line shows $\left(p_{1}, p_{2}\right)=(0.3,0.7)$, the dash-dot line $\left(p_{1}, p_{2}\right)=(0.2,0.8)$ and the solid line $\left(p_{1}, p_{2}\right)=(0.1,0.9)$. [Source: the author.]

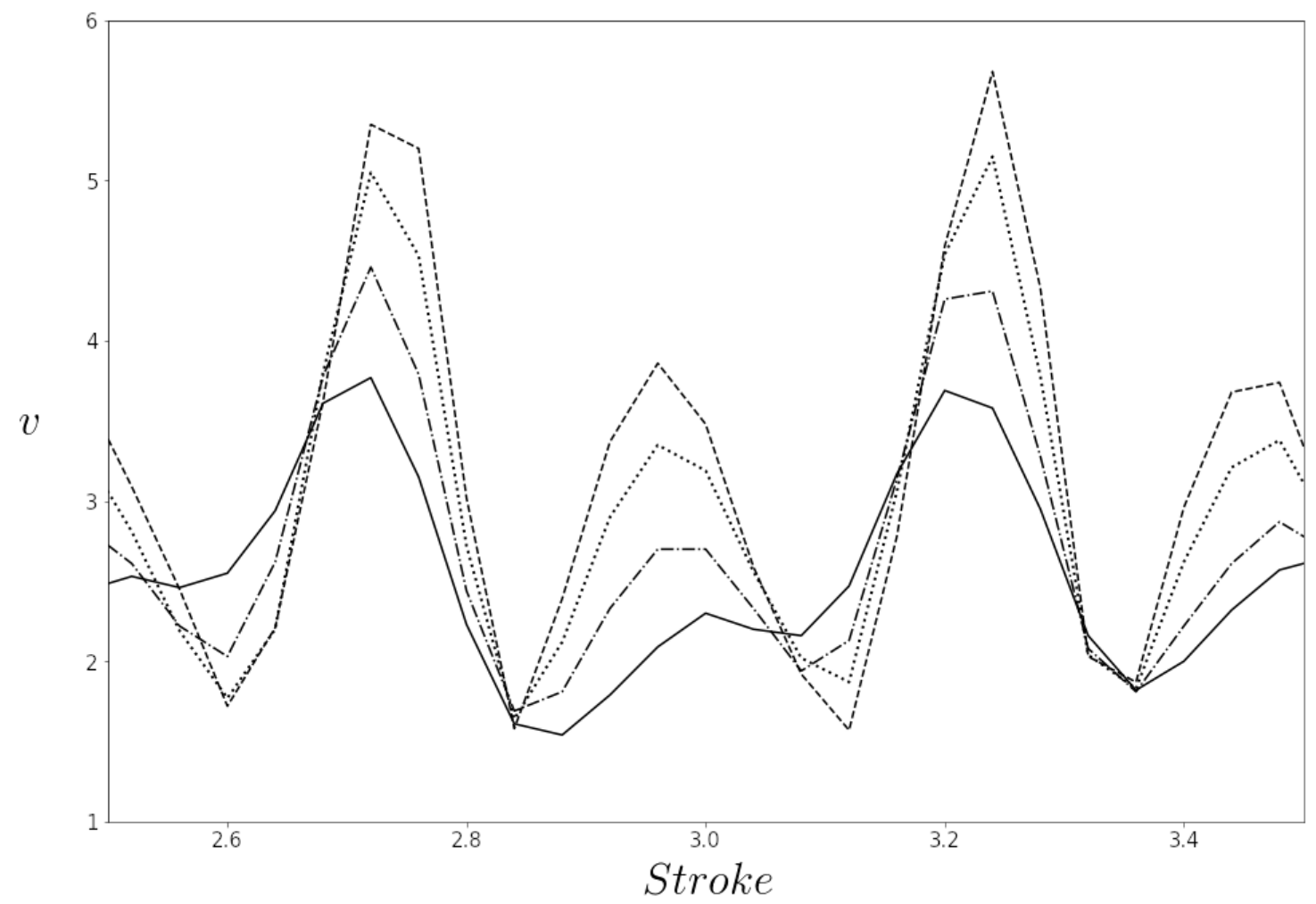


Figure 5.9: The trajectory of the nematode in the $x y$ space measured in bodylenghts. The first plot shows the trajectory for $\left(p_{1}, p_{2}\right)=(0.1,0.9)$, the second $\left(p_{1}, p_{2}\right)=(0.2,0.8)$, the third shows $\left(p_{1}, p_{2}\right)=(0.3,0.7)$ and the last represents $\left(p_{1}, p_{2}\right)=(0.4,0.6)$. [Source: the author.]
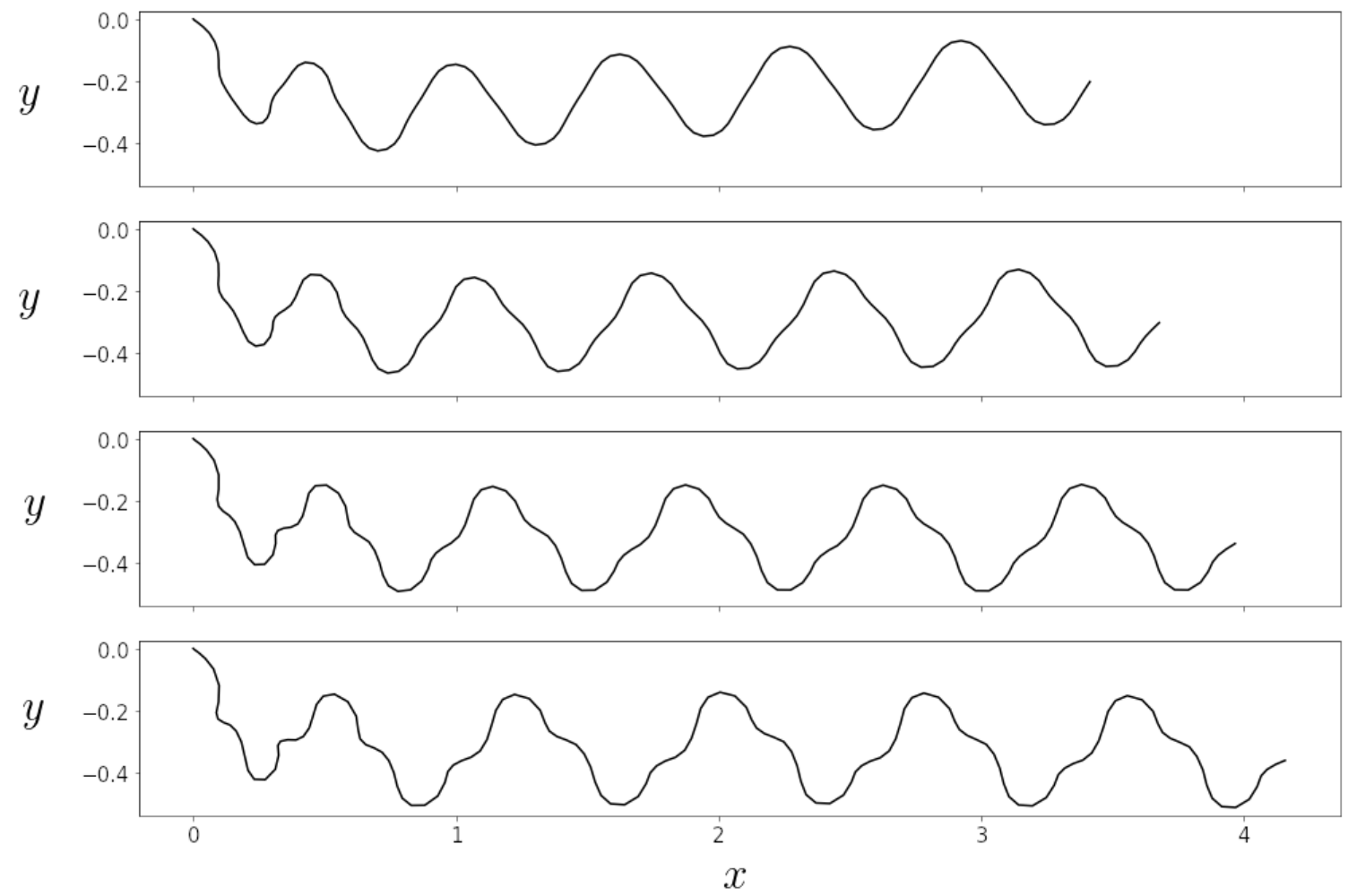


\subsubsection{Asymmetrical changes}

The interpolation points can also be changed asymetrically. Here, we consider the following cases:

$$
\begin{aligned}
& \left(p_{1}, p_{2}\right)=(0.1,0.9) \\
& \left(p_{1}, p_{2}\right)=(0.1,0.7) \\
& \left(p_{1}, p_{2}\right)=(0.1,0.5) \\
& \left(p_{1}, p_{2}\right)=(0.1,0.3)
\end{aligned}
$$

It is important to note that in this section, although we are asymmetrically varying $p_{2}$ about the interpolation interval, both the upstroke and downstroke have the same period. The only difference is that the rate of change of the interpolation function during each portion of the stroke. This change however leads to completely different results. Figure 5.10 shows the body curvature of the nematode depending on $\left(p_{1}, p_{2}\right)$.

Figure 5.10: Nematode curvature from $t=0$ time steps to $t=90$ time steps. The black nematode represents $\left(p_{1}, p_{2}\right)=(0.1,0.3)$, the red nematode $\left(p_{1}, p_{2}\right)=(0.1,0.5)$, the blue $\left(p_{1}, p_{2}\right)=$ $(0.3,0.7)$ and the green $\left(p_{1}, p_{2}\right)=(0.1,0.9)$. [Source: the author.]

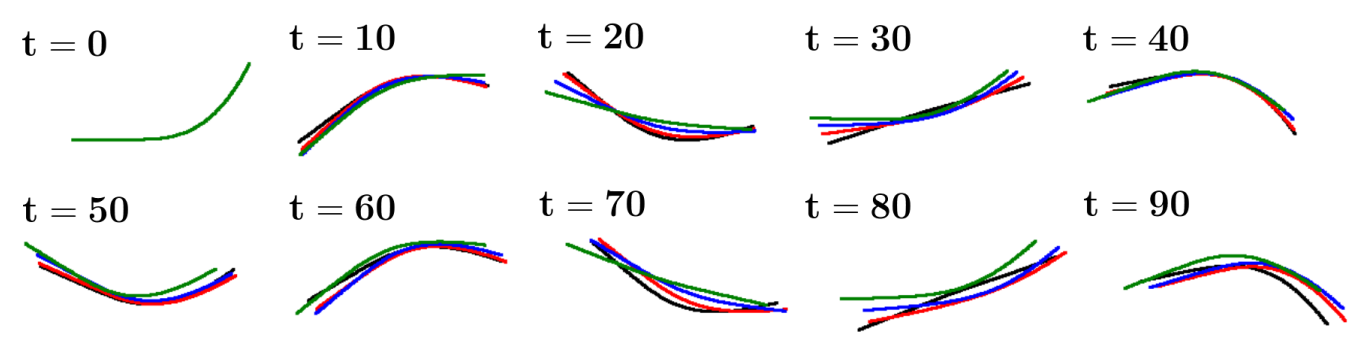

The dynamics are different between each swimmer for the above cases; however, perhaps surprisingly, there appears to be less variation than the previous case of symmetric $\left(p_{1}, p_{2}\right)$ choices in terms of forward swimming. Figures 5.11 and 5.12 show the distance and velocity considering this asymetric change. We observe that in this case there was a non-linear relationship with choice 
of $p_{2}$ and how fast the swimmer went, e.g., the case with $p_{2}=0.5$ was the fastest, followed by $p_{2}=0.7$, then 0.3 , and finally 0.9. A zoom of the forward velocity in bodylenghts per strokes vs. swimming strokes performed in the case of asymmetric interpolation is shown in figure 5.13.

Figure 5.11: Forward distance swam in bodylenghts vs. swimming strokes performed in the case of asymmetric interpolation points $\left(p_{1}, p_{2}\right)$. The dotted line represents $\left(p_{1}, p_{2}\right)=(0.1,0.5)$, the dash-dot line $\left(p_{1}, p_{2}\right)=(0.1,0.7)$, the dashed line $\left(p_{1}, p_{2}\right)=(0.1,0.3)$ and the solid line $\left(p_{1}, p_{2}\right)=$ $(0.1,0.9)$. [Source: the author.]

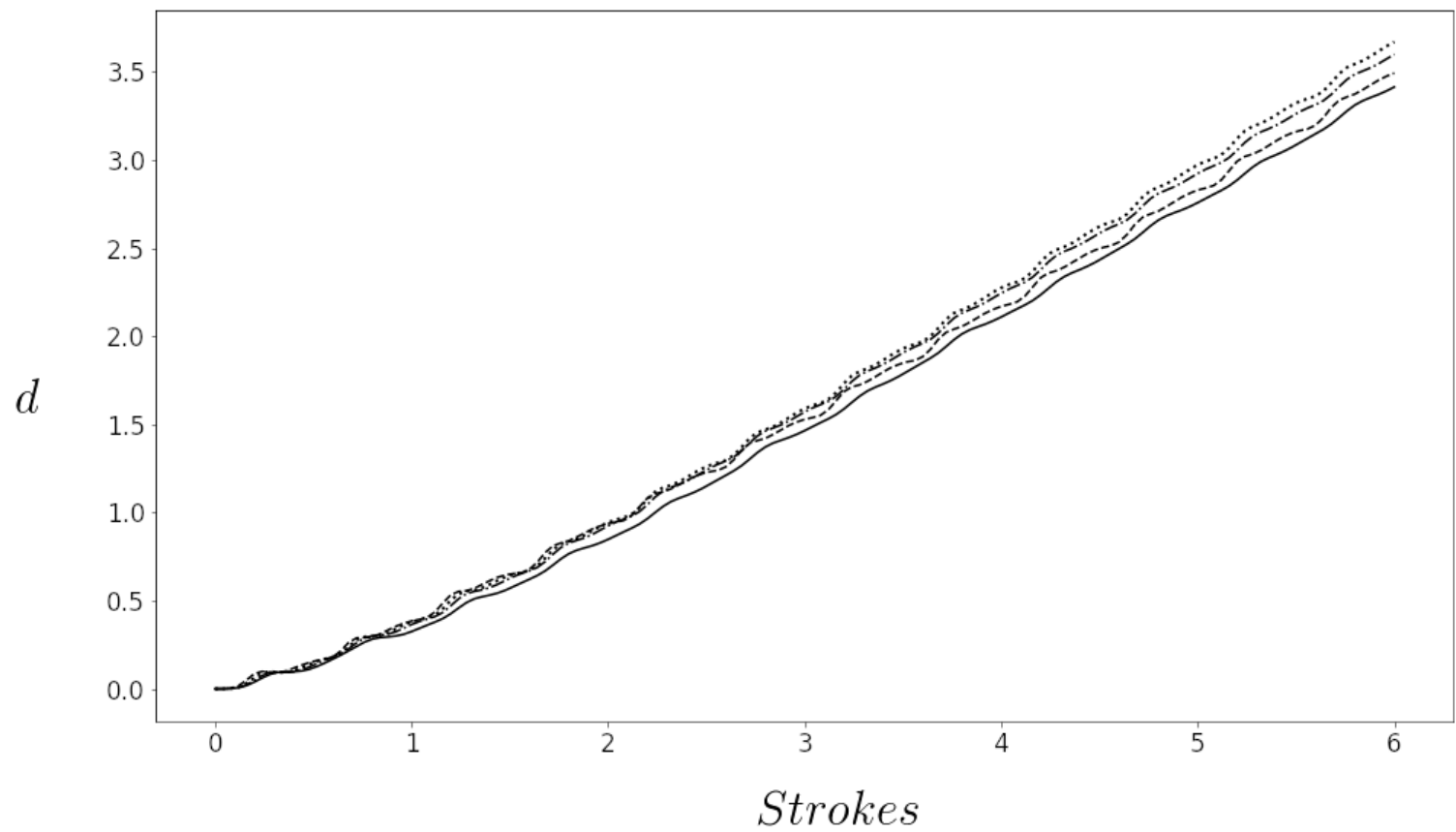

Figure 5.14 shows the trajectory of the nematode considering these asymetric changes. The asymmetry causes a difference in the trajectory. However, it is perceived that there is a large difference between these results and the experimental results observed. It is possible to notice that, despite the numerical capacities of this algorithm, for nematodes with no mutation, the simulations with symmetric changes in $p_{1}$ and $p_{2}$ are more similar to the experimental observations. 
Figure 5.12: Forward velocity in bodylenghts per strokes vs. swimming strokes performed in the case of asymmetric interpolation points $\left(p_{1}, p_{2}\right)$. The first plot represents $\left(p_{1}, p_{2}\right)=(0.1,0.9)$, the second plot $\left(p_{1}, p_{2}\right)=(0.1,0.7)$, the third $\left(p_{1}, p_{2}\right)=(0.1,0.5)$ and the last plot $\left(p_{1}, p_{2}\right)=$ $(0.1,0.3)$. [Source: the author.]

$v$
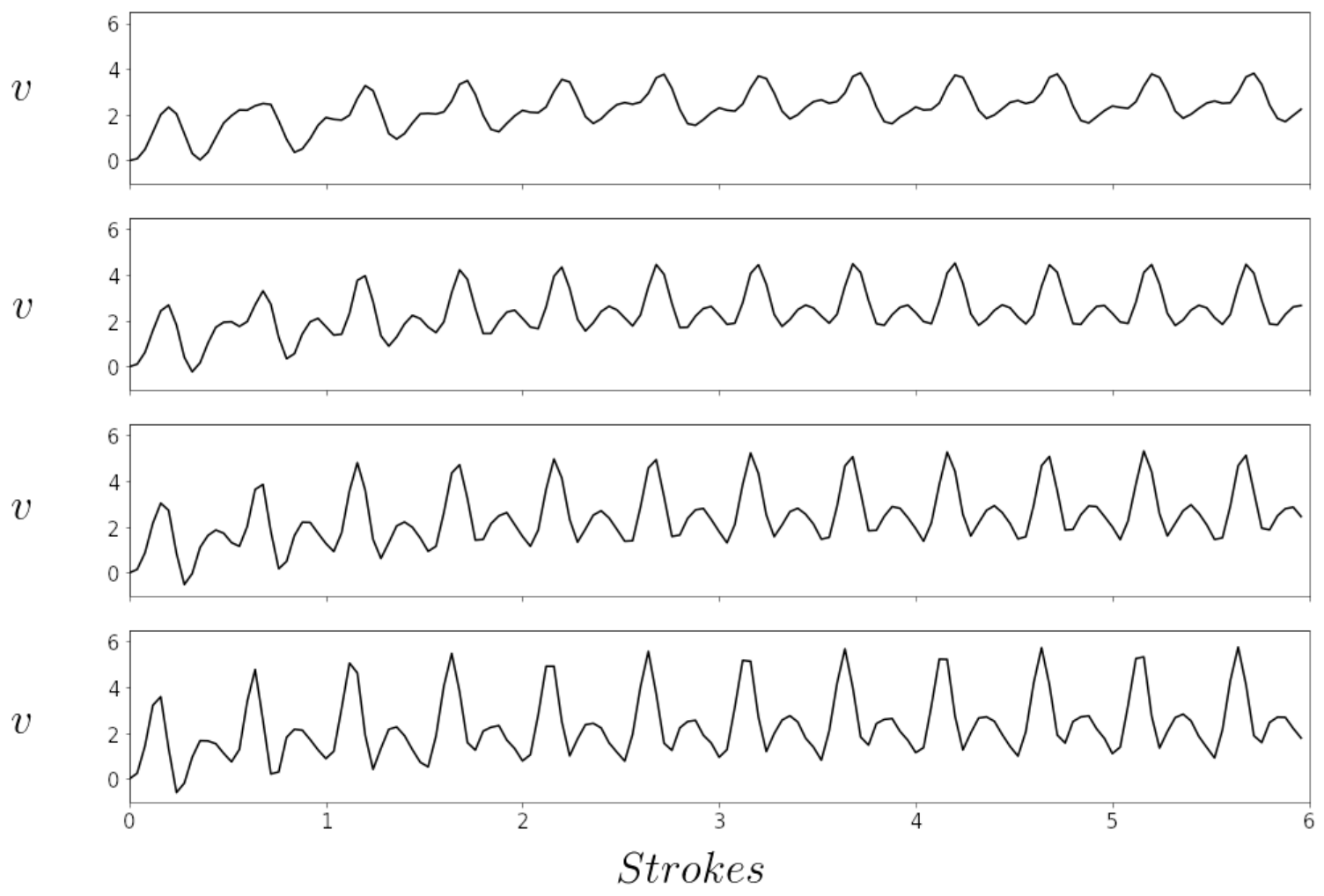
Figure 5.13: Zoom of the forward velocity in bodylenghts per strokes vs. swimming strokes performed in the case of asymmetric interpolation. The dashed line represents $\left(p_{1}, p_{2}\right)=(0.1,0.3)$, the dotted line shows $\left(p_{1}, p_{2}\right)=(0.1,0.5)$, the dash-dot line $\left(p_{1}, p_{2}\right)=(0.1,0.7)$ and the solid line $\left(p_{1}, p_{2}\right)=(0.1,0.9)$. [Source: the author.]

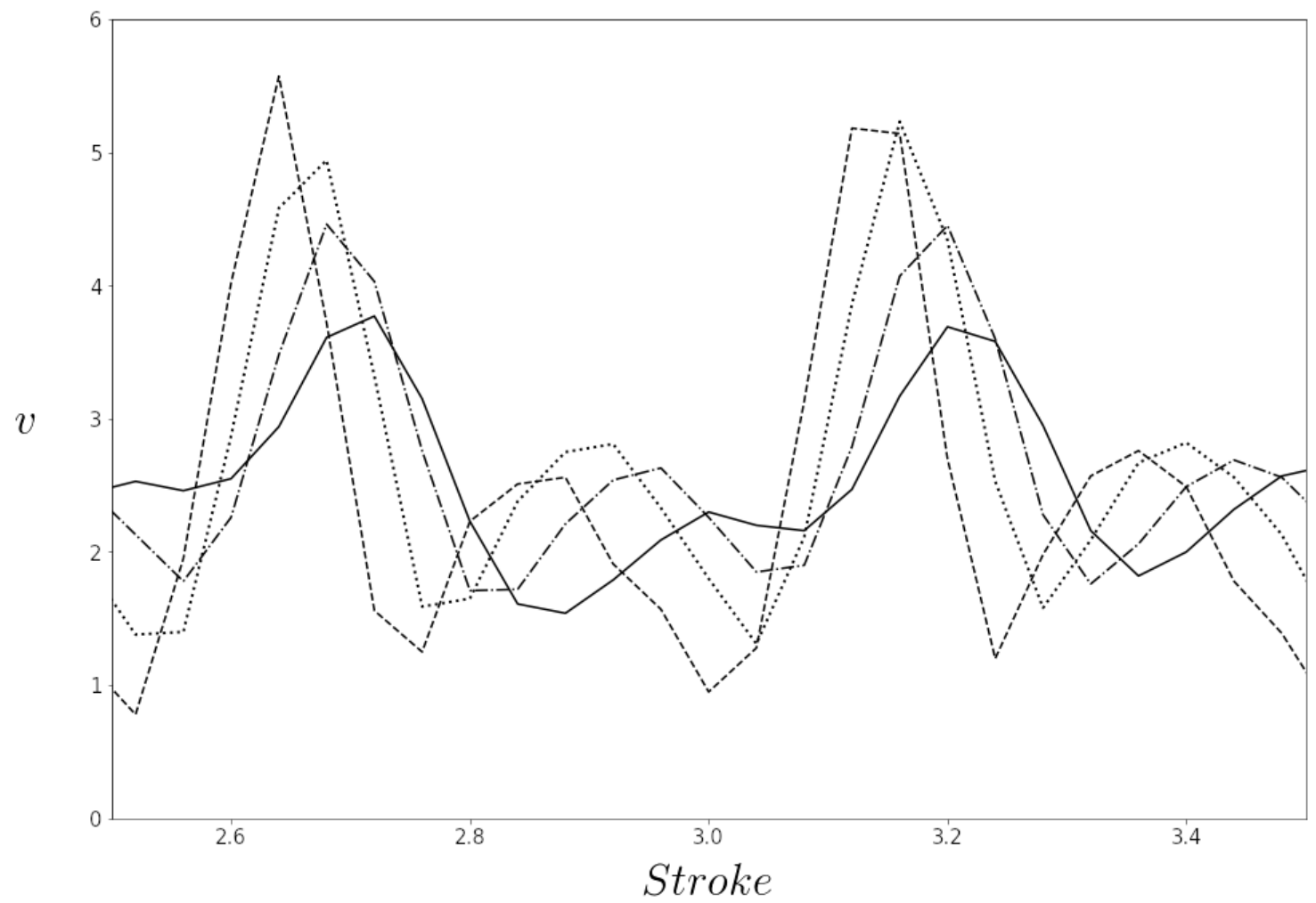


Figure 5.14: The trajectory of the nematode in the $x y$ space measured in bodylenghts. The first plot shows the trajectory for $\left(p_{1}, p_{2}\right)=(0.1,0.9)$, the second $\left(p_{1}, p_{2}\right)=(0.1,0.7)$, the third shows $\left(p_{1}, p_{2}\right)=(0.1,0.5)$ and the last represents $\left(p_{1}, p_{2}\right)=(0.1,0.3)$. [Source: the author.]
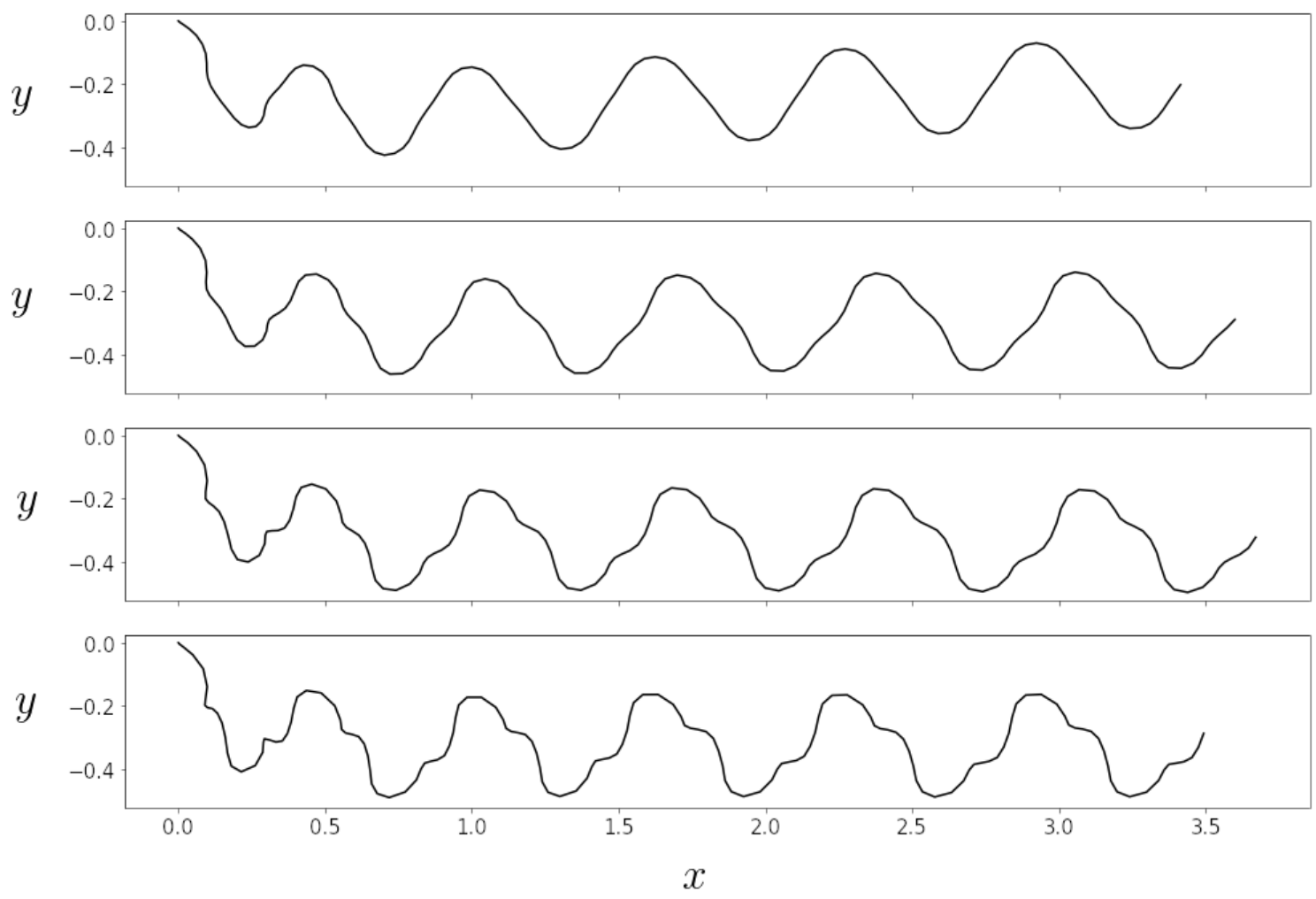


\subsubsection{Asymmetric Stroke Period}

The stroke period can also be changed, regardless of the interpolation points (kept as $(p 1, p 2)=(0.1,0.9))$. The upstroke $t_{u}$ and the downstroke $t_{d}$ percentages of the total stroke period were asymetrically varied. The following cases were simulated:

$$
\begin{array}{r}
t_{u}=t_{d} \text { representing } t_{u}=0.5 T \text { and } t_{d}=0.5 T \\
t_{u}=0.75 t_{d} \text { representing } t_{u}=0.429 T \text { and } t_{d}=0.571 T \\
t_{u}=0.5 t_{d} \text { representing } t_{u}=0.33 T \text { and } t_{d}=0.66 T \\
t_{u}=0.25 t_{d} \text { representing } t_{u}=0.2 T \text { and } t_{d}=0.8 T
\end{array}
$$

Figure 5.15: Nematode curvature from $t=0$ time steps to $t=90$ time steps. The black nematode represents $t_{u}=0.25 t_{d}$, the red nematode $t_{u}=0.5 t_{d}$, the blue $t_{u}=0.75 t_{d}$ and the green $t_{u}=t_{d}$. [Source: the author.]
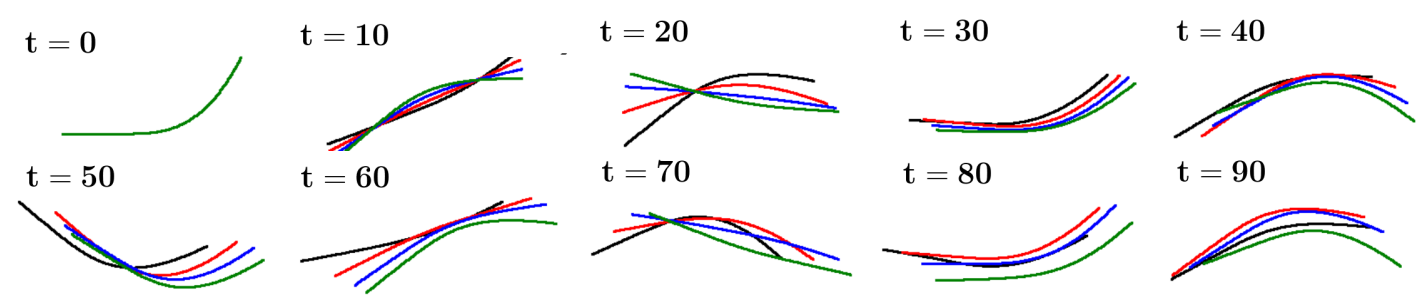

Figure 5.15 shows the body curvature of the nematode depending on $t_{u}$ and $t_{d}$. As the upstroke percentage of a stroke decreases, it happens faster. However, although the swimmer that moves forward the fastest has the quickest upstroke, having a faster upstroke does not always equate to a faster forward swimming speed. Interestingly, due to the asymmetric upstroke (UPS) and downstroke (DWS), the swimming velocity profiles look significantly different. In particular, the waveforms appear trimodal rather than bimodal, which were observed in the cases of varying the interpolation modes. Figures 5.16 and 5.17 show the forward distance and velocity considering asymetric UPS and DWS and figure 5.18 represents a zoom in the forward velocity. 
Figure 5.16: Forward distance swam in bodylengths vs. swimming strokes performed in the case of asymmetric upstroke and downstroke periods. The dashed line represents UPS $=25 \%$ DWS, the dotted UPS $=50 \%$ DWS, the dash-dot UPS $=75 \%$ DWS and the solid UPS $=100 \%$ DWS. [Source: the author.]

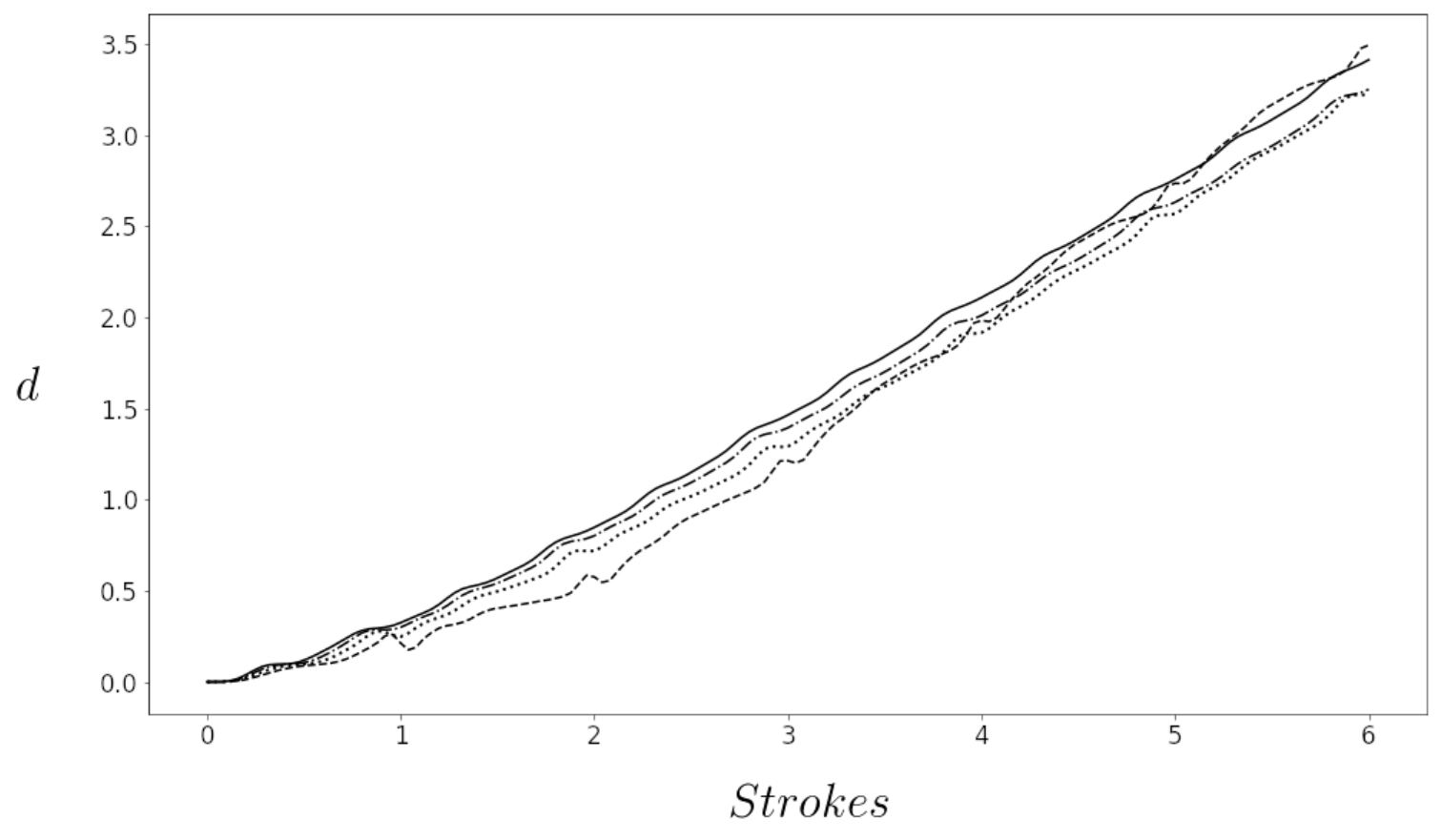


Figure 5.17: Forward velocity in bodylengths per stroke vs. swimming strokes performed in the case of asymmetric upstroke and downstroke periods. The first graph represents UPS $=100 \%$ DWS, the second UPS $=75 \%$ DWS, the third UPS $=50 \%$ DWS and the last UPS $=100 \%$ DWS. [Source: the author.]

$v$

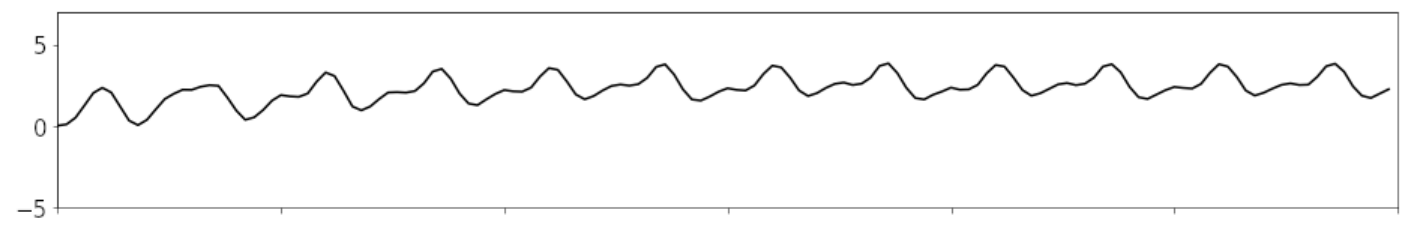

$v$

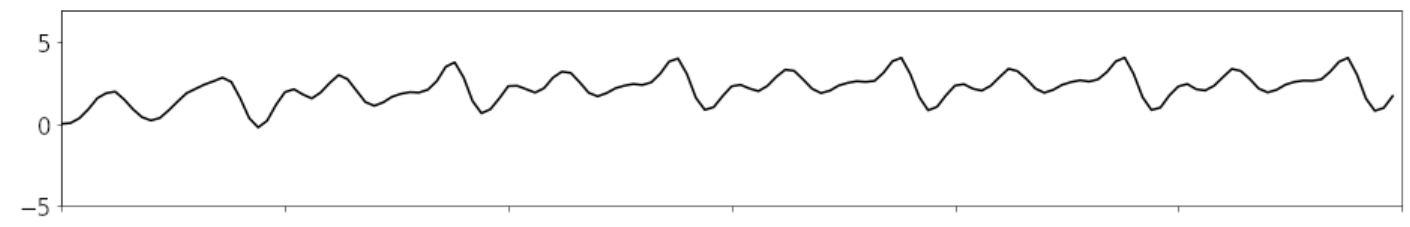

$v$

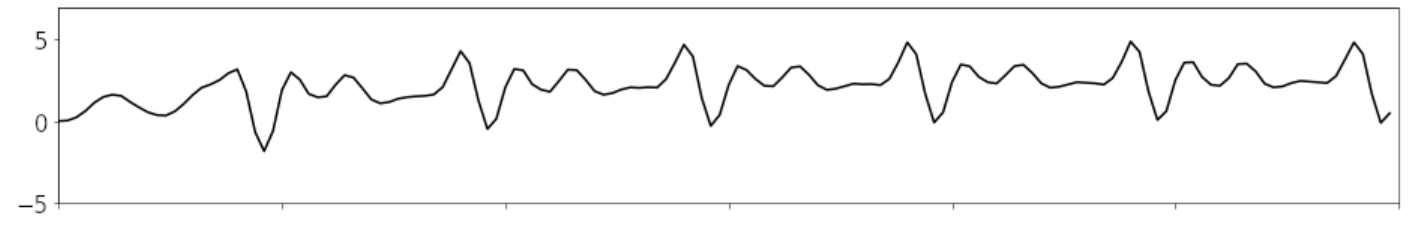

$v$

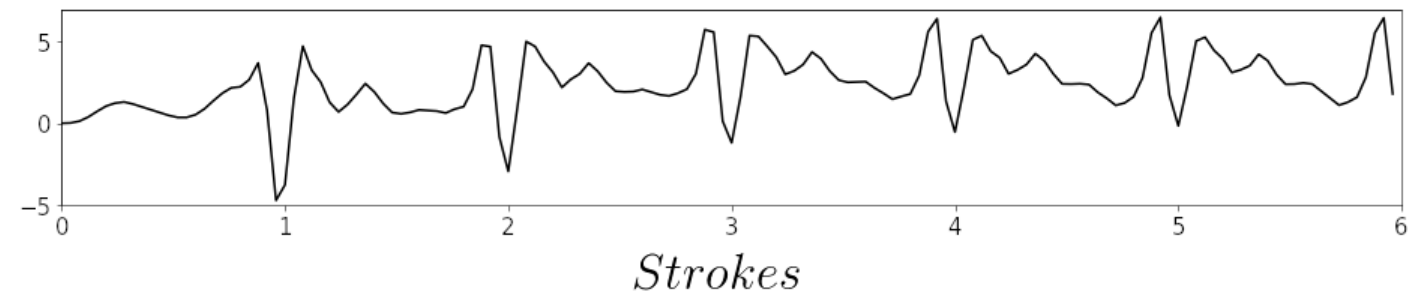


Figure 5.18: Zoom of the forward velocity in bodylenghts per strokes vs. swimming strokes performed in the case of asymmetric upstroke and downstroke periods. The dashed line represents UPS $=25 \%$ DWS, the dotted line shows UPS $=50 \%$ DWS, the dash-dot line UPS $=75 \%$ DWS and the solid line UPS $=100 \%$ DWS. [Source: the author.]

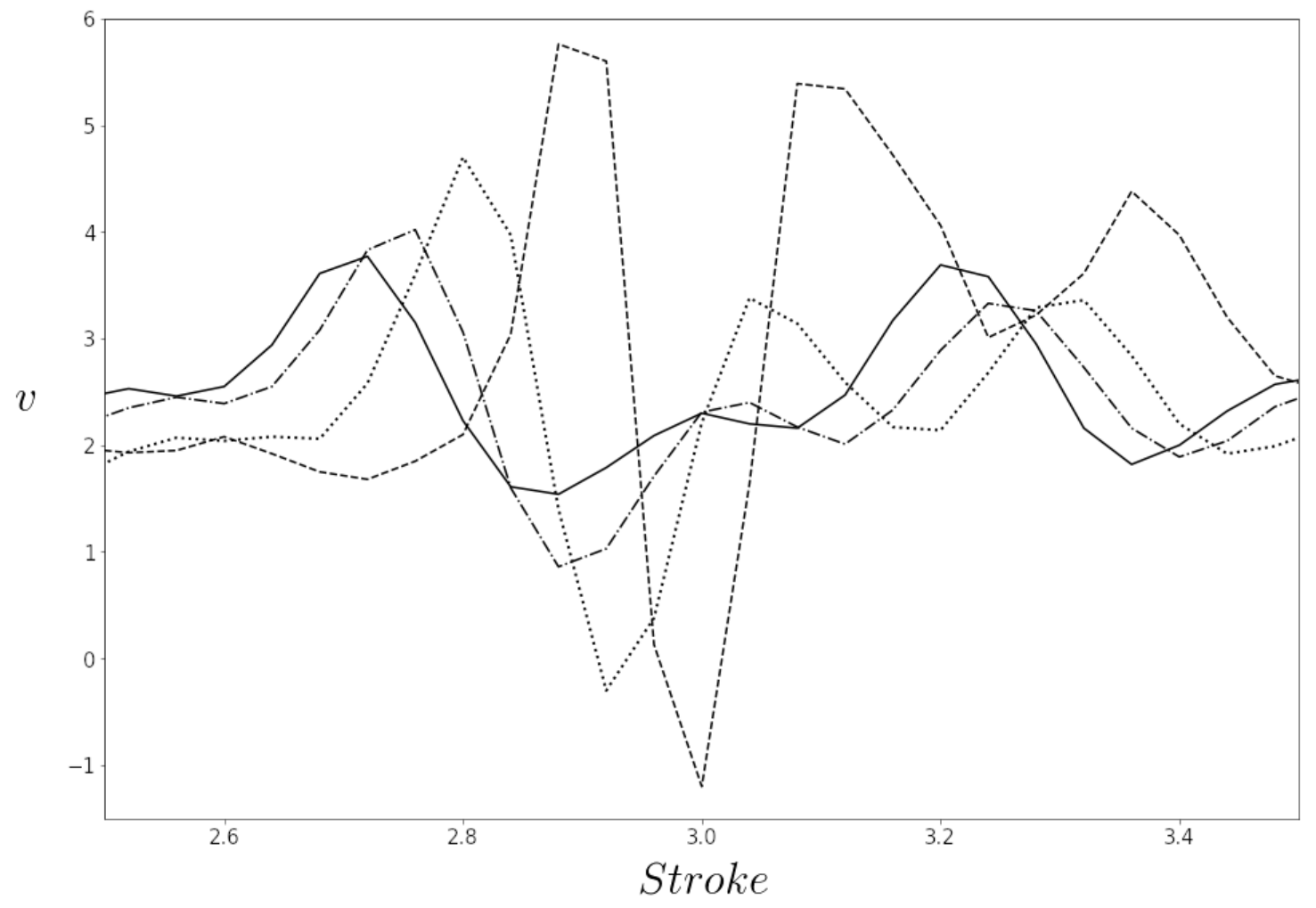


Figure 5.19: The trajectory of the nematode in the $x y$ space measured in bodylenghts. The first plot shows the trajectory for UPS $=100 \%$ DWS, the second UPS $=75 \%$ DWS, the third shows UPS $=$ $50 \%$ DWS and the last represents UPS $=25 \%$ DWS. [Source: the author.]
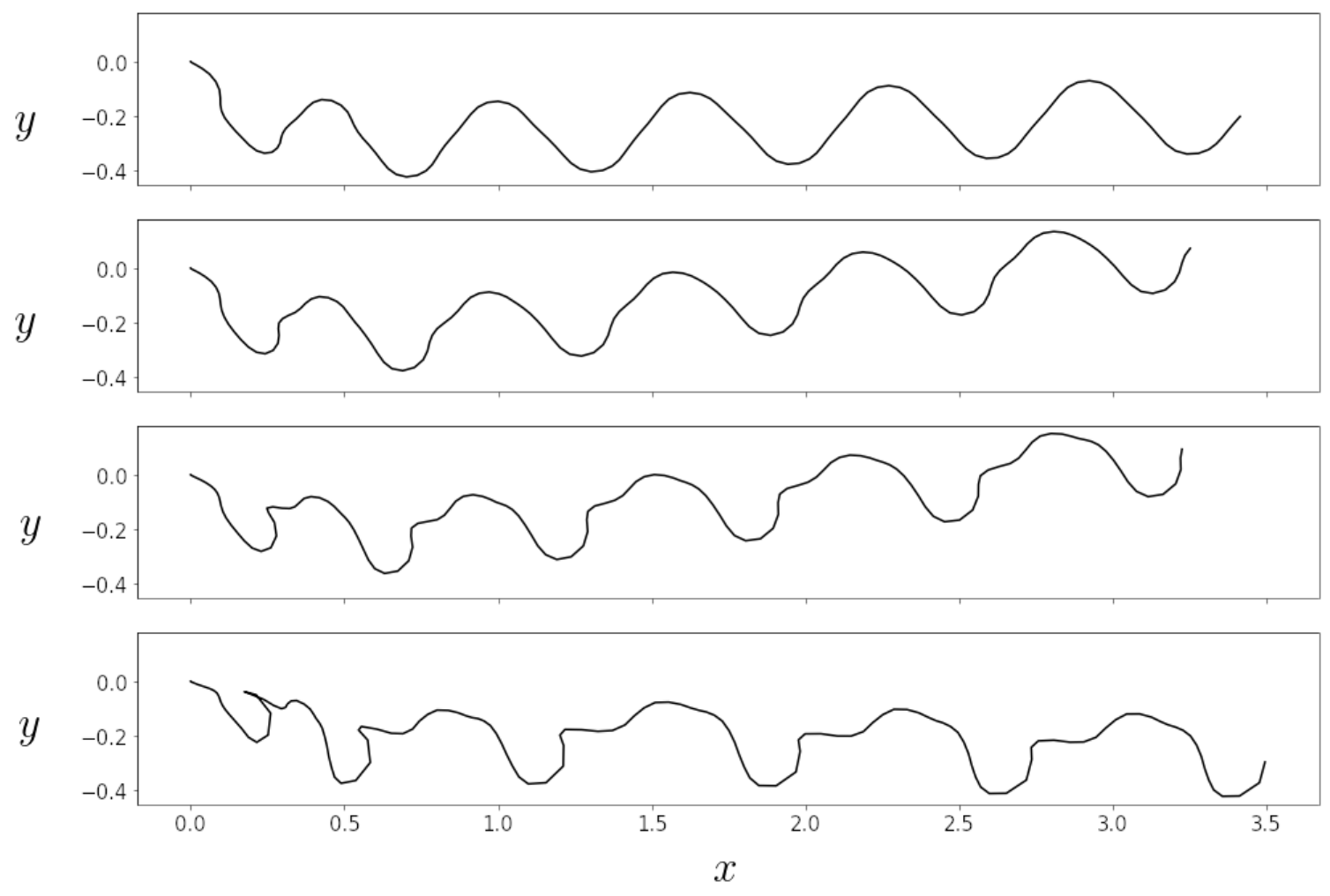


\subsection{Chapter conclusions}

In the previous chapter, it was observed that the nematode relaxation function is directly related to its orientational behavior. In order to simplify the rheological model that will be proposed in the next chapter, immersed boundary simulations of an orientational flexible filament were performed and the results were compared to the kinetic results presented in chapter 3 . The observed results allowed us to relate the curvature of the nematode to its velocity and acceleration conditions.

The interpolation median points used were varied symmetrically and asymmetrically. In this sense, it can be seen from figures 5.20 and 5.21 that the velocity and acceleration profiles change abruptly.

Figure 5.20: Plots of the piecewise cubic interpolant, $g(t)$, its derivative, $\dot{g}(t)$, and its second derivative, $\ddot{g}(t)$, with $0 \leq t \leq 1$, for varying $\left(p_{1}, p_{2}\right)$ symmetrically chosen. The blue lines are represented by $\left(p_{1}=0.1, p_{2}=0.9\right),\left(p_{1}=0.2, p_{2}=0.8\right)$ the red lines, $\left(p_{1}=0.3, p_{2}=0.7\right)$ the black lines and $\left(p_{1}=0.4, p_{2}=0.6\right)$ the green lines. [Source: the author.]
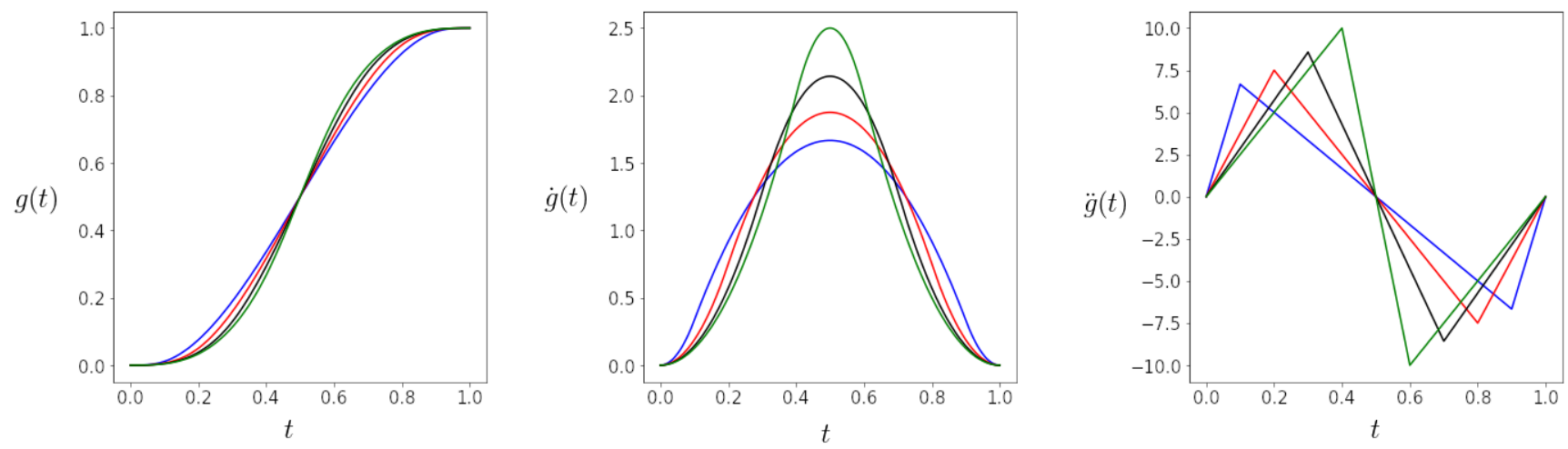

Figure 5.20 shows exactly why when $\left(p_{1}=0.4,0.6\right)$ the nematode moved more with less strokes. The acceleration, $\ddot{g}(t)$ has the highest value in both phases of curvature. On the other hand, when the mediary points were asymmetrically modified, as shown in figure 5.21, the two cases where there is greater symmetry between the acceleration and velocity of both curvature phases are represented by the red and black lines. In this sense, there is an optimization between the symmetry of the derivatives and their real magnitude. It is possible to observe that even when not prescribing the precise movement of a swimmer, but rather the microorganism's material property states (e.g., curvature), changing the spline interpolant affects the system's outcome. 
Figure 5.21: Plots of the piecewise cubic interpolant, $g(t)$, its derivative, $\dot{g}(t)$, and its second derivative, $\ddot{g}(t)$, with $0 \leq t \leq 1$, for varying $\left(p_{1}, p_{2}\right)$ asymmetrically chosen. The blue lines are represented by $\left(p_{1}=0.1, p_{2}=0.9\right),\left(p_{1}=0.1, p_{2}=0.7\right)$ the red lines, $\left(p_{1}=0.1, p_{2}=0.5\right)$ the black lines and $\left(p_{1}=0.1, p_{2}=0.3\right)$ the green lines. [Source: the author.]
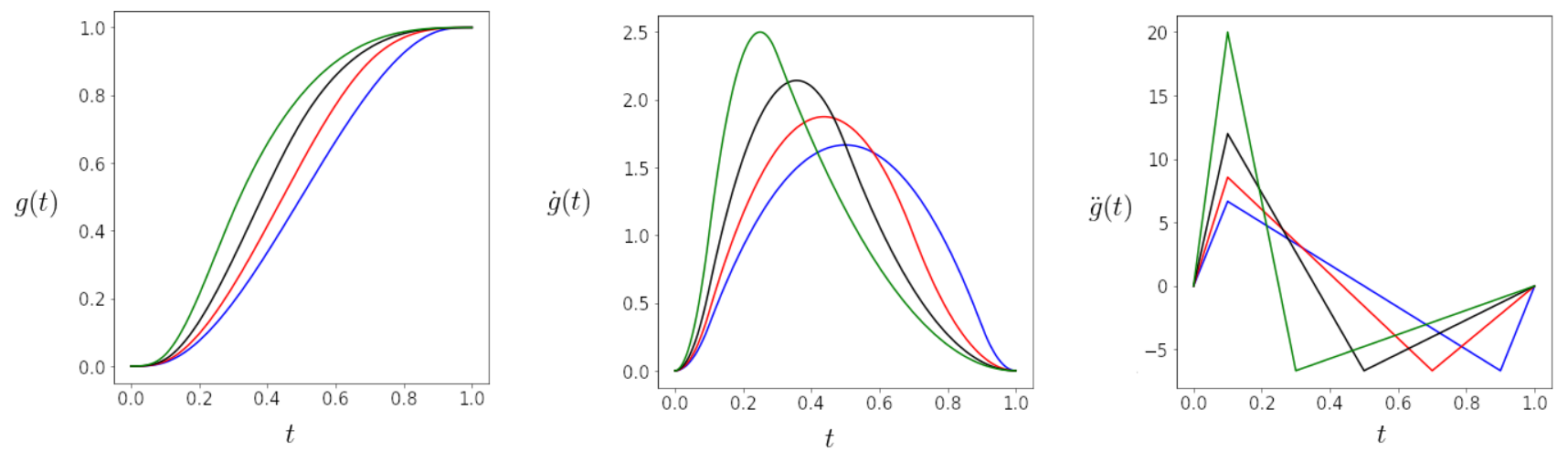

In addition, it was observed that a simple flexible filament presents results similar to those observed in kinematic experiments. In general, for the purpose of locomotion, the viscoelastic characteristics of the nematodes themselves do not seem to interfere severely. In this sense, this information will be used to construct the rheological model in the next chapter. 


\section{Nonlinear Constitutive Model}

This chapter provides a constitutive model for the active suspension of nematodes, which is lacking in the literature. The new model is tested on both linear and nonlinear regimes and compared with the experimental data, showing promissing results.

Considering the orientational instabilities of the active suspension observed in the experiments, a nonlinear phenomenological model for neutrally buoyant force-free active suspension of nematodes is proposed and tested on the description of the linear and nonlinear viscoelastic behavior of this living suspension. The model is based on coarse grained equations that govern the motion of the nematic living particles over suficiently large length and time scales for the continuum description of the motion to be valid. In a nematic model, it is assumed that interactions among the cells are such that they maintain a common alignment so that the orientations are all close to the director orientation. Therefore, it is not necessary to include an orientation distribution.

The central idea behind the modeling of the active nematic is that out of thermal equilibrium, new terms enter the equation of motion for the nematic director as well as the stress tensor. These terms are more relevant than the terms mandated by thermodynamic approaches, in the sense that their effects are stronger at long wavelengths and at long times, i.e. in the thermodynamic limit.

As stated before, the average equations for the balance of mass, accounting for the incompressibility of the medium and the balance of forces in the absence of fluid inertia are, respectively:

$$
\begin{gathered}
\nabla \cdot \boldsymbol{u}=0, \\
-\nabla p+\nabla \cdot \boldsymbol{T}=0,
\end{gathered}
$$

where $\boldsymbol{u}$ denotes the ensemble-average velocity, $p$ denotes the mechanical pressure and $\boldsymbol{T}$ is the suspension bulk stress written in terms of two contributions: one due to the viscous ambient fluid, $\boldsymbol{D}$, and another due the activity particles $\boldsymbol{\sigma}$, as 


$$
\boldsymbol{T}=2 \mu \boldsymbol{D}+\boldsymbol{\sigma}
$$

where $\sigma$ represents the active bulk stress tensor due to particle stress contribution, given by

$$
\boldsymbol{\sigma}=-\phi \beta\left(\boldsymbol{Q}-\frac{1}{3} \operatorname{tr}(\boldsymbol{Q}) \boldsymbol{I}\right)
$$

Here $\mu$ is the viscosity of the ambient fluid, $\boldsymbol{D}=(1 / 2)\left[\nabla \cdot \boldsymbol{u}+(\nabla \cdot \boldsymbol{u})^{T}\right]$ is the average rate of deformation tensor, $\phi$ is the particle volume fraction and $\beta$ is an important parameter which characterizes the strength of the elementary particle force dipole exerted on the solvent fluid and its sign has vital rheological consequences.

Particle activity enhaces effective viscosity if $\beta<0$ (i.e. puller) and reduces it if $\beta>0$ (pusher), as observed in previous experiments. Now, the alignment order deviatoric tensor $\boldsymbol{Q}$ in our model is defined such as described by Simha (SIMHA AND RAMASWAMY, 2002):

$$
\boldsymbol{Q}=<\boldsymbol{q}_{k} \boldsymbol{q}_{k}-\frac{1}{3} \boldsymbol{I}>
$$

where $\boldsymbol{q}_{k}$ is the unit vector along the axis of the $k$ th active particle and represents the particle axisdirection (or director field), $\boldsymbol{I}$ is the identity tensor and $\langle\cdot\rangle$ denotes an orientational ensembleaverage. Therefore, the order tensor $Q$ represents an average of the direction of the director field. In this research, $Q$ is a smooth field but not nearly isotropic everywhere as considered in the lineaized model.

In order to provide a closure for the model, a time evolution equation for the order tensor $Q$ is needed. As a preliminary model, we use Oldroyd- Maxwell upper convected material derivative for a dilute active suspension in the absence of thermal or active fluctuations. Under this assumption, the evolution of $Q$ can be first described by

$$
\frac{\partial \boldsymbol{Q}}{\partial t}=\lambda \boldsymbol{D}-\frac{1}{\tau_{a}} \boldsymbol{Q}+\left(\nabla \boldsymbol{u} \cdot \boldsymbol{Q}+\boldsymbol{Q} \cdot \nabla \boldsymbol{u}^{T}\right)-(\boldsymbol{u}+U \boldsymbol{n}) \cdot \boldsymbol{Q}
$$


where $\partial / \partial t$ denotes an Eulerian time derivative, $\nabla \boldsymbol{u}$ is the ensemble-average velocity gradient tensor and $\tau_{a}$ is the activity correlation time related to the particle orientation. Also, $\lambda$ is an independent reactive coefficient which characterizes the reversible response of the director field to symmetric local stress (FORSTER, 1974).

According to this model, $Q$ can be convected with the sum of the free active particle velocity $U \boldsymbol{n}$ and the fluid velocity $\boldsymbol{u}$ and can also be changed due to particle relaxation (or tumbling), rotation and stretching, because of the vorticity and the rate of deformation produced by the ensembleaveraged fluid velocity field. The signature of this constitutive equation is a shear stress that does not vanish for zero deformation rate. This is because the active filament system is being driven out of equilibrium by two sources of energy: one external, due to the shear, and the other internal, due to the activity.

\subsection{Non-affine Motion}

Most theories of soft matter elasticity assume that the local strain in a sample after deformation is identical everywhere and equal to the macroscopic strain, or equivalently that the deformation is affine. Recently, also the importance of non-affine deformations has been pointed out (HEUSSINGER AND FREY, 2006). We observed that smaller nematodes frequently undergo reversible but highly non-affine motion when sheared. In this sense, no result in the literature proposes such a complete model for the study of nematode suspensions.

While the nematodes centers follow the macroscopic shear deformation affinely, the head and tail can deviate from the affine deformation field in order to minimize the global free energy (HinsCH AND FREY, 2009). Also, the entanglement of nematodes (Rousslet et al., 2014) can react by non-affine deformation to the macroscopic shear.

In cross-linked polymeric fluids, macroscopic deformation of the medium induces two motions at the micro-level: (i) sliding of junctions with respect to their reference positions that reflects non-affine deformation of the network, and (ii) slippage of chains with respect to entanglements that is associated with unfolding of back-loops (DROZDOV AND GOTTLIEB, 2005). Similar behavior was observed with C. elegans. In this condition, we rewrite equation 6.6 using Gordon-Schowalter derivative which takes non-affine motion into account: 


$$
\frac{D \boldsymbol{Q}}{D t}=\lambda \boldsymbol{D}-\frac{1}{\tau_{a}} \boldsymbol{Q}+\boldsymbol{Q} \cdot(\nabla \boldsymbol{u}-\xi \boldsymbol{D})^{T}+(\nabla \boldsymbol{u}-\xi \boldsymbol{D}) \cdot \boldsymbol{Q}
$$

where $\xi$ is interpreted as the representative of the slip of the nematode with respect to the neighboring continuum. The special case when $\xi=0$ corresponds to the upper-convected derivative (Deville AND GATSKY; SARAmito, 2012; 2014). However, this slip parameter should only be considered when the system is not diluted and there are entanglements of nematodes, as seen in figure 6.1, obtained from the experiments.

Figure 6.1: Entanglement of nematodes observed during experiments after shear. [Source: the author.]

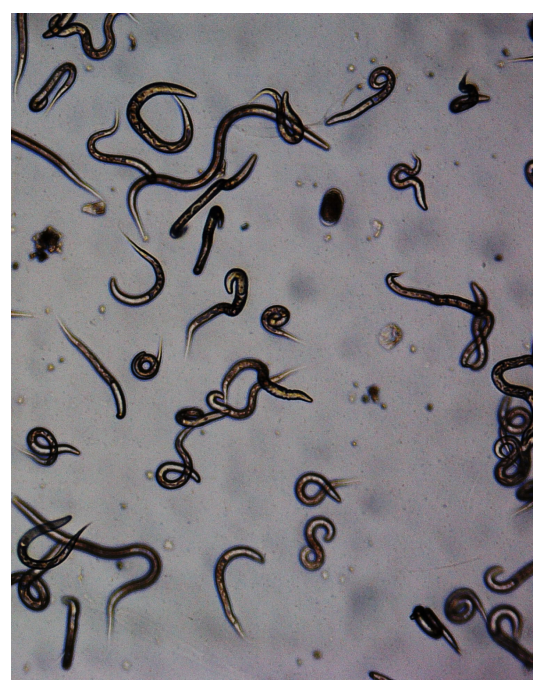

At this point it is useful to reiterate the definitions of two commonly used dimensionless numbers to describe flow, the Deborah number $D e=\tau_{c} \omega$ and the Weissenberg number $W i=\tau_{c} \omega \gamma_{0}=\tau_{c} \dot{\gamma}$. The Deborah number is defined as the ratio of a characteristic relaxation time of the material $\tau_{c}$ and a characteristic time of observation $\tau_{0}$, which is inverse of the angular frequency $\tau_{0}=1 / \omega$ for oscillatory shear. It measures to what degree elastic effects influence the overall mechanical response. For example in the Maxwell model, if $D e<1$ the viscous behavior dominates, $G^{\prime \prime}>G^{\prime}$, whereas for $D e>1$, the response is predominantly elastic, $G^{\prime}>G^{\prime \prime}$. The Weissenberg number, is the ratio of $\tau_{c}$ and a characteristic time $\tau_{d}$ of the deformation. In this case, $\tau_{d}$ is the inverse of the shear rate $\tau_{d}=1 / \dot{\gamma}$ for steady shear or $\tau_{d}=1 /\left(\omega \gamma_{0}\right)$ for oscillatory shear. The Weissenberg number can be seen as a dimensionless shear rate that indicates the importance of nonlinear behavior. That means the input flow is defined as $\dot{\gamma}_{0}=W i \cos (D e t)$. 
Figures 6.2 and 6.3 show the difference from the elastic and viscous Lissajous-Bowditch curves when non-affine motion is considered or not. It is possible to observe that when the volume fraction is small, the response change due to the presence of the slip parameter is not representative. However, as the volume fraction increases and the collective behavior starts to play a role, the presence of the slip parameter dramatically changes the behavior of the suspension.

The concentration dependence of large amplitude oscillatory shear (LAOS) behaviour would be due to the increase in the length of the tubular chains, and the consequent increase in the entanglements at higher concentrations. The inverse frequency dependence is due to the fact it competes with the relaxation processes in the material. When the frequency is small, lesser than the inverse of the relaxation time, the material has enough time to relax within the period of shear leading to secondary loops. 


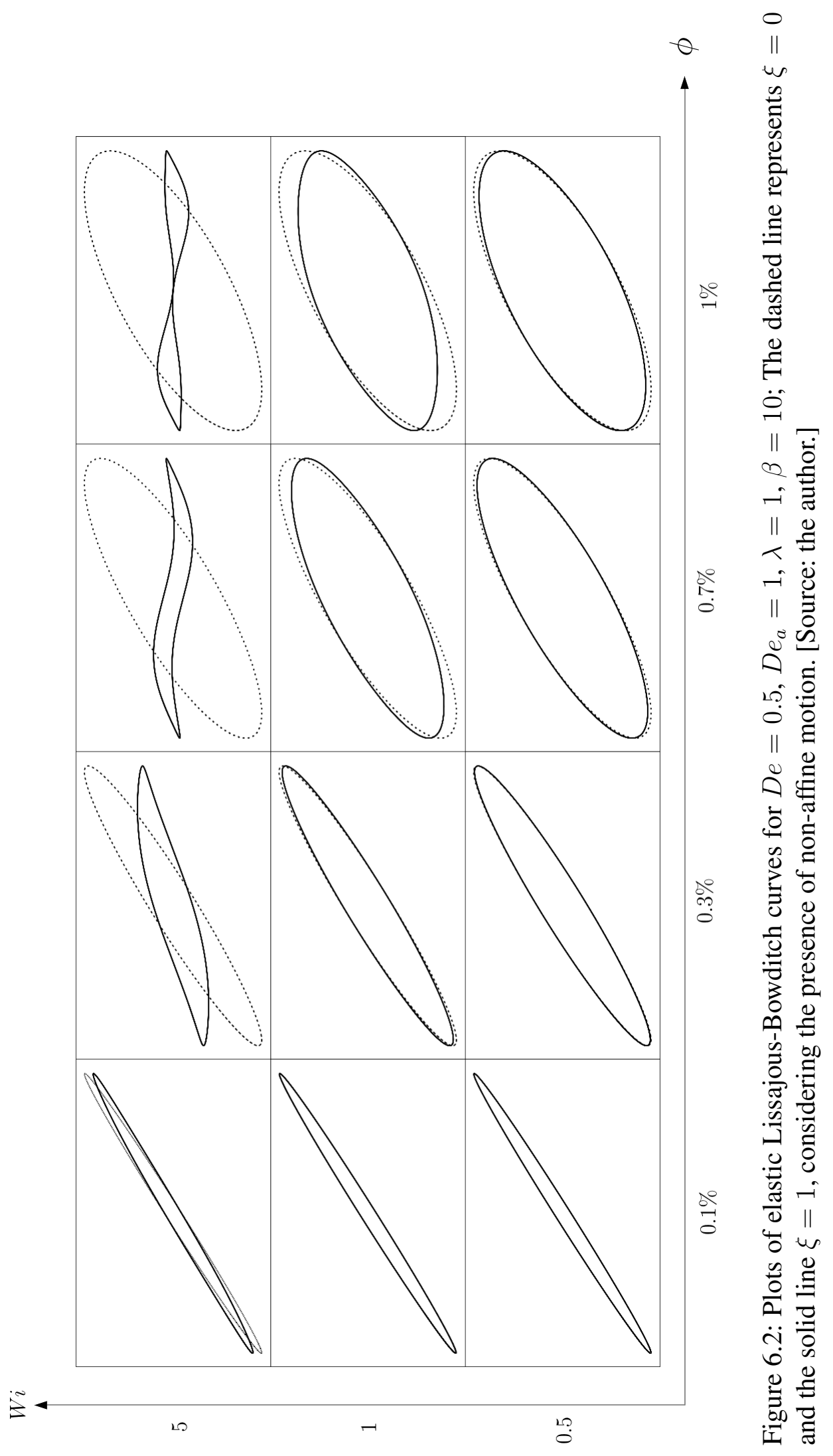




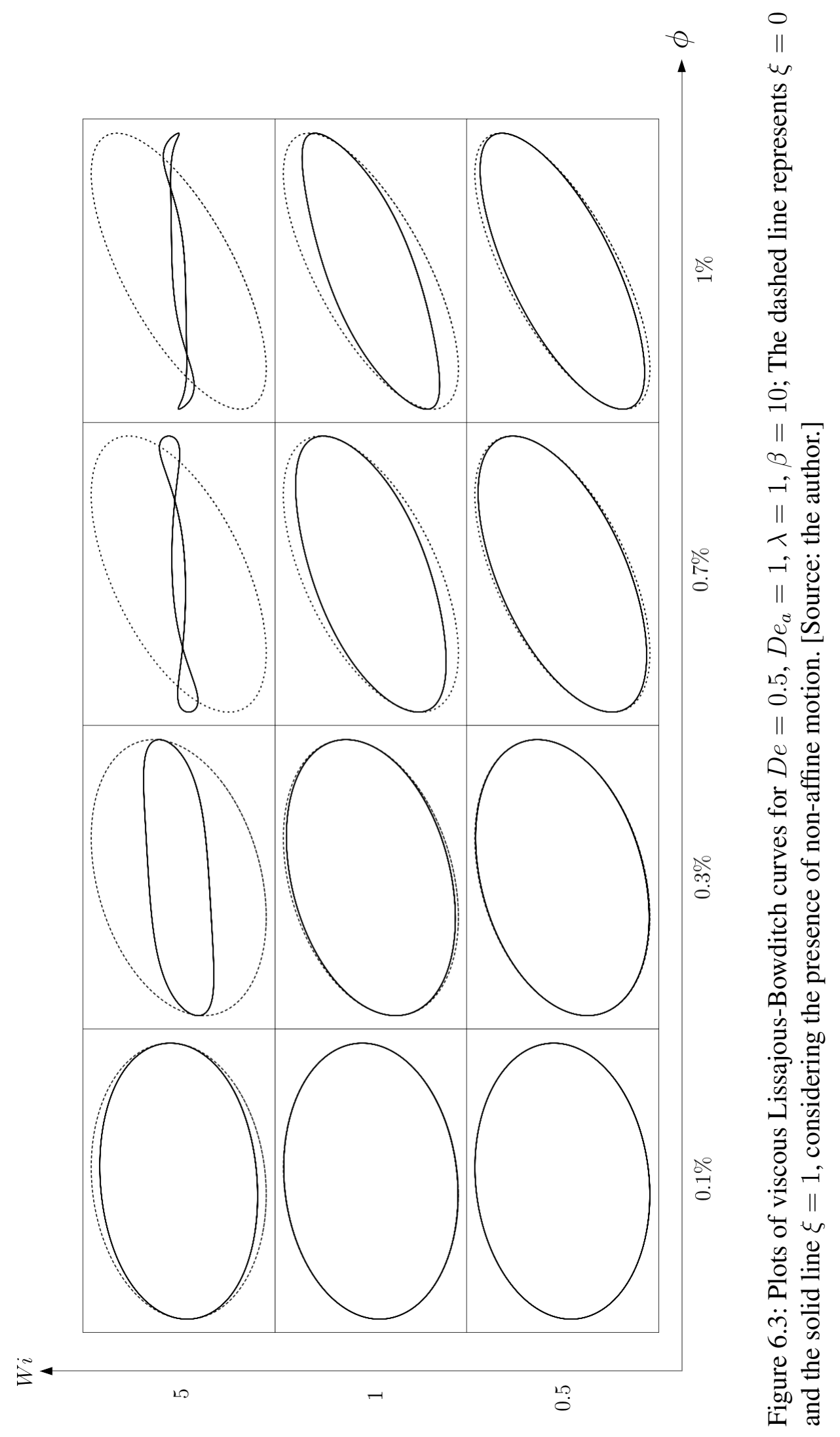


Using Gordon-Schowalter equations allows us to observe that shear and normal stress differences are oscillatory functions of shear strain (AGASSANT AND PIAU; LARSON, 1996; 1988). The shear stress and elastic recovery oscillate between positive and negative values. An oscillatory response osccurs not only in step shears but also in start-up of steady shearing, if the amplitude of the forcing becomes large.

Usually, LAOS is focused on the long-time steady-state oscillatory material response that is represented by a closed space-curve. However time-varying material responses associated with thixotropy, shear-induced migration or rheological aging can also be represented in this material phase-space by trajectories that slowly decay towards the corresponding periodic attractor (EWOLDT AND MCKInLEY, 2010).

\subsection{Linear Regime}

In order to compare our model with the small amplitude oscillatory shear results, one can rewrite the Fourier components which are in-phase and out-phase with the strain input.

$$
\sigma(t)=\gamma_{0}\left(G^{\prime}(\omega) \sin (\omega t)+G^{\prime \prime}(\omega) \cos (\omega t)\right)
$$

being $G^{\prime}$ and $G^{\prime \prime}$ the viscoelastic moduli. In the linear regime, the elastic Lissajous curve will be elliptical as shown in figure 6.4. This curve as a whole is tilted, with a slope corresponding to the elastic modulus $G^{\prime}(\omega)$ of the material. For a linear material, this slope can either be taken from the line passing through the points of minimum and maximum strain or as the tangential curve to the Lissajous curve to zero strain, shown as a dashed line. Both slopes can be used as a definition of the characteristic elastic modulus. The area under the curve still corresponds to the dissipated energy per cycle and is completely characterized by the first harmonic of the stress response.

We now introduce an active Deborah number, $D e_{a}$, associated with $\tau_{a}$, the swimming timescale of the nematode. We may see how the activity timescale also influences the behavior of the suspension in figure 6.5. It is possible to see that as $D e_{a}$ increases, the resultant behavior approaches a pure viscous fluid. However, as it decreases the elastic behavior becomes pronounced due to the activity. As the amplitude of excitation increases, the nonlinearities also start to appear. 


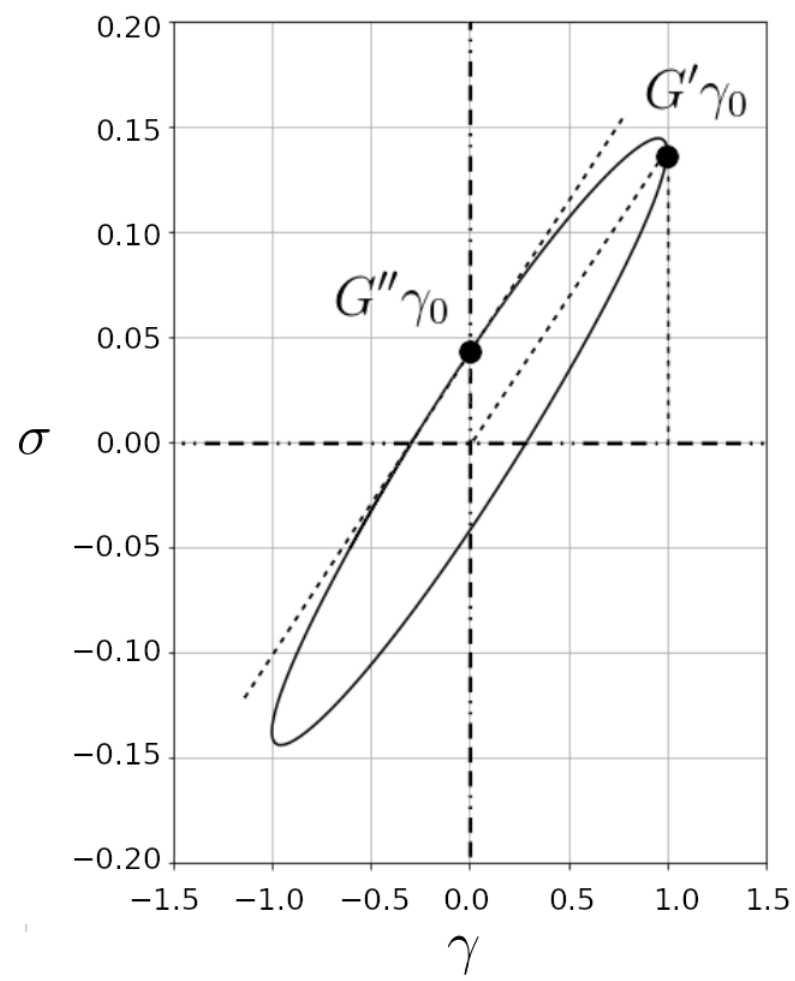

Figure 6.4: Plot of elastic Lissajous-Bowditch curve for $D e=1, D e_{a}=1, W i=0.15, \lambda=1$, $\beta=30, \xi=1$ and $\phi=0.5 \%$, showing $\sigma[\mathrm{Pa}]$ per strain, $\gamma$. [Source: the author.]

Considering the linear regime and the small amplitude oscillatory shear tests, we were able to compare the model to the experimental data. Figure 6.6 shows the stress obtained experimentally from the values of $G^{\prime}$ and $G^{\prime \prime}$ in comparison with the values predicted by the model. In this case, there are no major changes considering $\xi=0$ or $\xi=1$, since these are small-amplitude tests of a diluted system. 


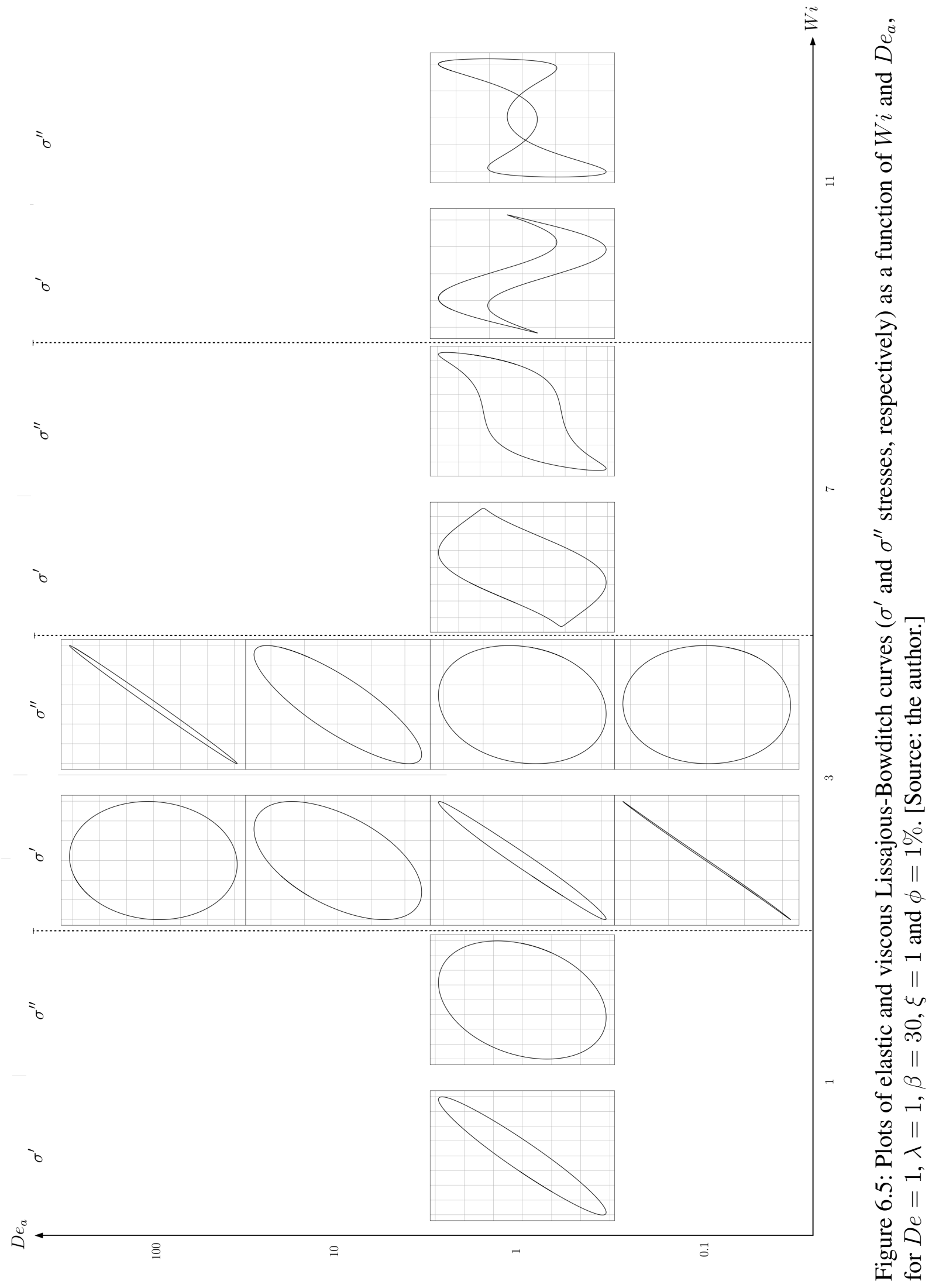



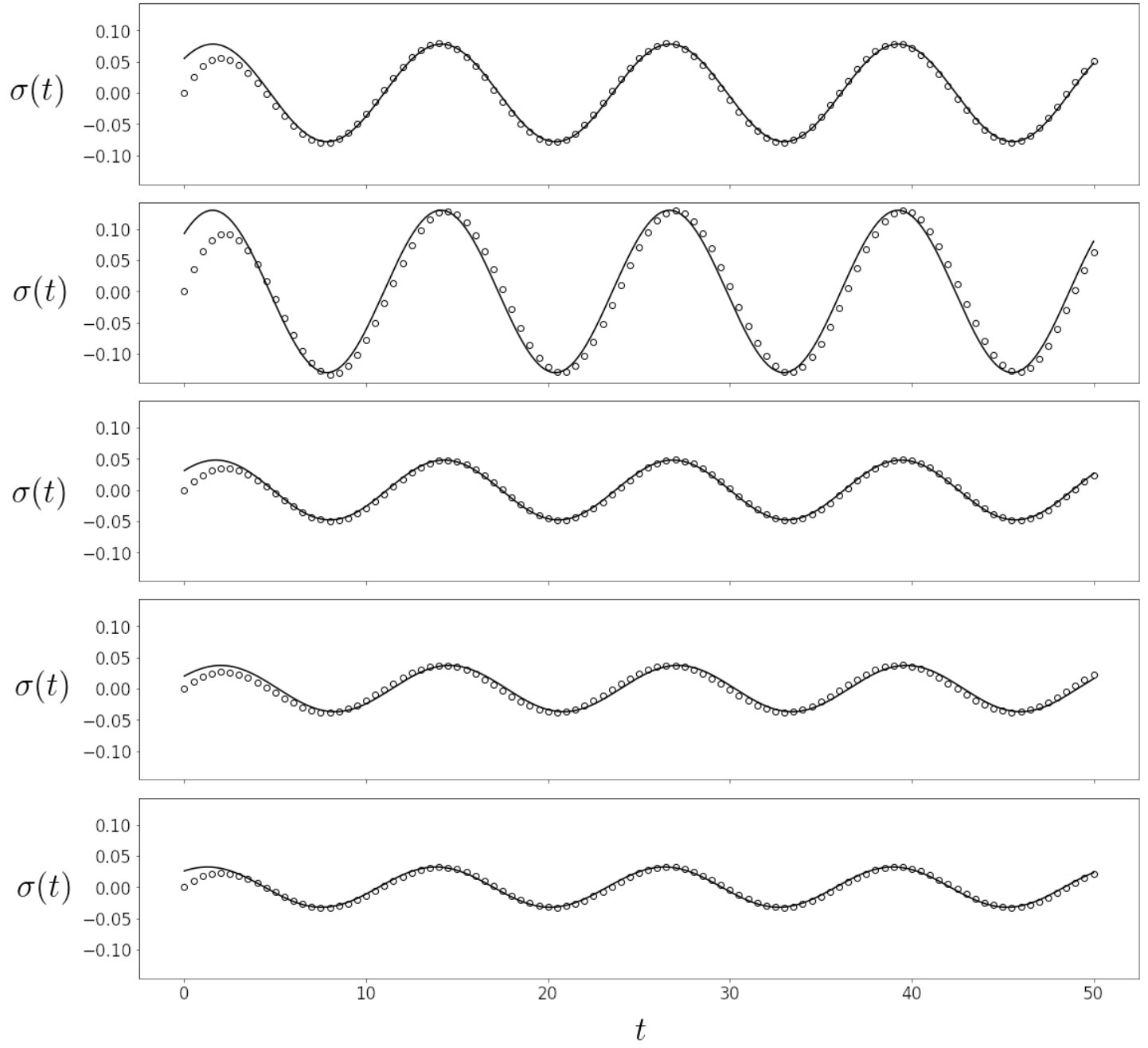

Figure 6.6: Stress, $\sigma[\mathrm{Pa}]$, as a function of time $[\mathrm{s}]$, obtained from the experimental data of $G^{\prime}$ and $G^{\prime \prime}$ (hollow circles) and from the proposed model (solid black line) for $\phi=0.001, \phi=0.002$, $\phi=0.003, \phi=0.004$ and $\phi=0.005$ respectively from top to botton. The parameters used to fit each curve are given in table 6.1. [Source: the author.] 
Table 6.1: Parameters used to fit the experimental data to the model, considering the actual experimental parameters $\gamma=0.4, \omega=0.5, \xi=1, \eta=0.01$. De $e_{a}$ was set to 1 , so that the time scales of the flow and activity become compatible. [Source: the author.]

\begin{tabular}{rcl}
\hline$\beta$ & $\phi$ & \multicolumn{1}{c}{$\lambda$} \\
\hline 580 & 0.001 & 0.95 \\
390 & 0.002 & 1 \\
200 & 0.003 & 0.6 \\
80 & 0.004 & 0.65 \\
170 & 0.005 & 0.35
\end{tabular}

The relative viscosity fit of the so-called "active elastic region" (ranging from $0.002 \lesssim \phi \lesssim$ 0.004) proposed in chapter 4 was also tested with the same values of $\beta$ from table 6.1, as shown in figure 6.7. Again, there is a decrease in the bulk relative viscosity $\eta_{r}$ in a non-linenar fashion. That means that the elastic effect of each particle becomes important and there is a maximum value for the energy production. As stated before, in this particle volume fraction range, particle activity relaxation is comparable to the applied flow. The non-dissipative energy production gave rise to negative viscosity values and the particle stresslet decreased. It is important to highlight that $\beta$ is directly related to the behavior of the particles as a whole. That is, considering that it is stresslet bulk, the larger the volume fraction, the greater the magnitude of $\beta$. In general, $\beta$ is associated not only with the type of microorganism, but also with its interactions. 
Figure 6.7: Relative bulk viscosity $\eta_{r}$ of the suspension as a function of the volume fraction, $\phi[\%]$. The hollow circles represent the viscosity obtained assuming the $\beta$ values from the table 6.1, used to fit the model to the experimental data. The dashed line represent the spline adjusted from the experiments. [Source: the author.]

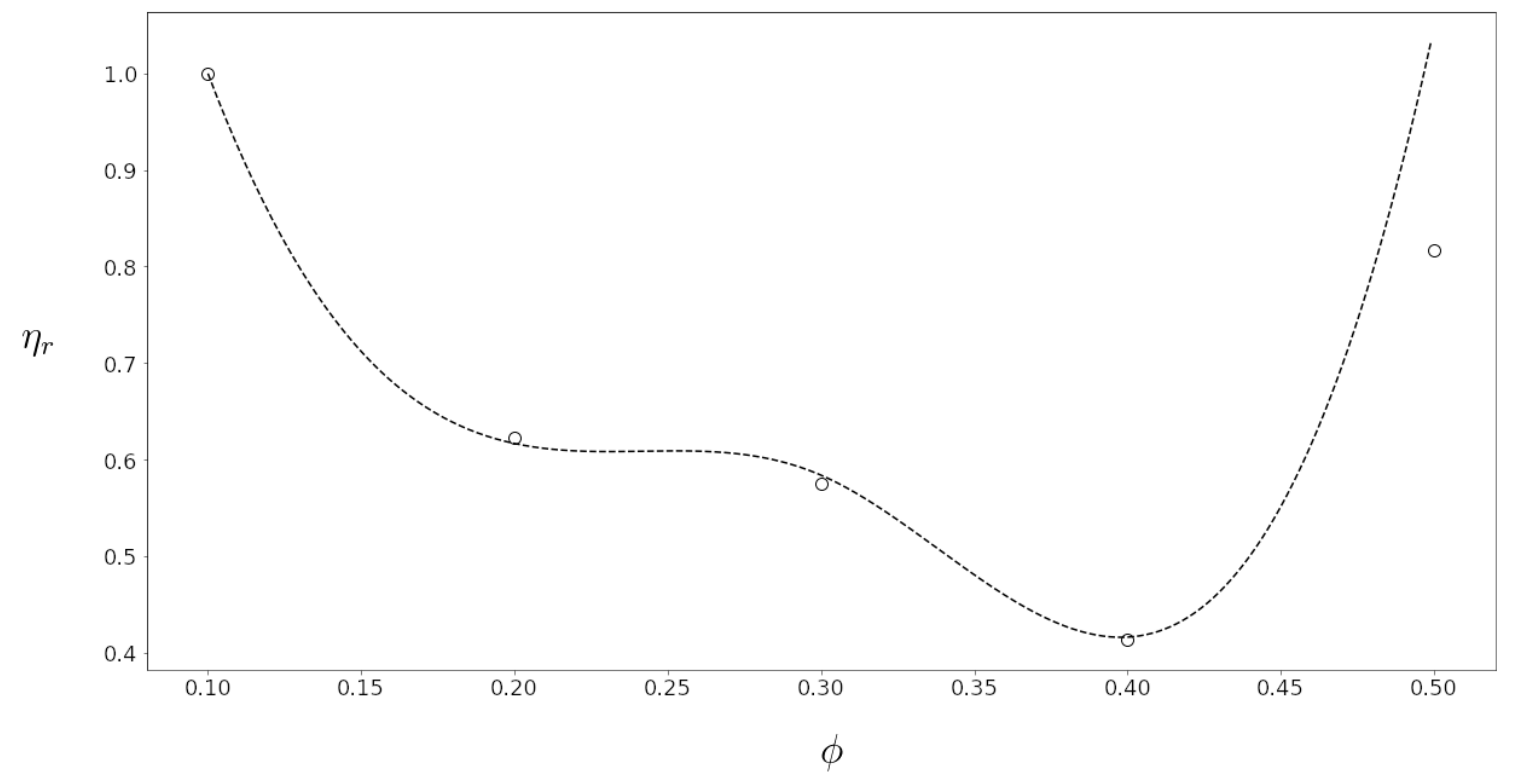




\subsection{Nonlinear Regime}

Now, the model can be tested for large amplitudes and frequencies. Using the LissajoudBowditch approach explained in chapter 2, we are now able to draw the Pipkin diagram of the fluid. The Pipkin diagram is used to analyze how the fluid changes its behavior depending on the applied amplitude and frequency. At low frequencies and strain amplitudes, the material response is purely viscous and Newtonian. As mentioned earlier, this is at timescales larger than the largest relaxation time in the material. With an increase in frequency, we observe viscoelastic response at timescales smaller than the largest relaxation time in the material. Depending on the strain amplitude or strain rate amplitude, a crossover from linear viscoelastic behavior to nonlinear viscoelastic behavior is observed. At low frequencies, this crossover occurs beyond a threshold of strain rate amplitude, while at high frequencies, it occurs beyond a threshold of strain amplitude. At very high frequencies, with timescales shorter than the smallest relaxation time of the material, all modes are frozen and an elastic response is observed.

In general, the projections of nonlinear responses will not entirely be positively oriented (where positive orientation is defined by the right-hand rule) nor will they be simple closed curves; they will contain alternating regions of positive and negative orientation and will intersect themselves multiple times. As a result, the nonlinear parameter must be defined by only one cycle of the signal at steady state.

In figures 6.10 and 6.11, at sufficiently low Deborah number, the viscoelastic material has enough time to relax and behaves like a Newtonian liquid for small amplitudes. Increasing $W i$ at constant $D e$ means applying higher shear rates that result in nonlinear effects such as shear thinning, which can be modeled by a generalized Newtonian fluid model. Increasing $W i$ at high but constant $D e$ drives the material first into the instrinsic LAOS region.

The distinct non-ellipsoidal wave forms can be interpreted using the sequence of physical processes approach following Rogers (ROGERS AND LETTINGA, 2012). In figure 6.8, starting at zero stress $\left(\gamma_{0}=-\gamma_{0}\right)$ and observing the response to increasing strain (reading the LissajousBowditch figure from left to right), the stress increases almost linearly with strain as depicted by the portions of the Lissajous curve which are straight in the elastic representation in figure 6.8. It is associated with the cage modulus or residual modulus $G_{r}=\left.\frac{d \sigma}{d \gamma}\right|_{\sigma=0}$. This modulus quantifies the strenght of the residual elasticity in the linear region after reversal of flow direction that is recovered 
in every cycle. For small deformations, that is the linear regime, $G_{r}$ is equal to the storage modulus $G^{\prime}$ (KIND et al., 2015). Once the yield stress, shown by the dashed line in the same figure, is exceeded, the material flows with a plastic viscosity which is seen in the non-monotonic portion of the stress trajectory in the viscous representation. This continues until the end of the half-cycle ( $\gamma=+\gamma_{0}, \dot{\gamma}=0$ ), and subsequently the sequence is repeated in the opposite direction. A similar behavior is observed in figure 6.9.

Figure 6.8: Plots of elastic ( $\left.\sigma^{\prime}[\mathrm{Pa}]\right)$ and viscous $\left(\sigma^{\prime \prime}[\mathrm{Pa}]\right)$ Lissajous-Bowditch curves as a function of $\gamma$ and $\dot{\gamma}\left[\mathrm{s}^{-1}\right]$ for $W i=5, D e=0.5, D e_{a}=1, \lambda=1, \beta=30, \xi=1$ and $\phi=1 \%$. The yielding point is represented by $\gamma_{y}$ and the maximum stress is $\sigma_{\max }$. [Source: the author.]
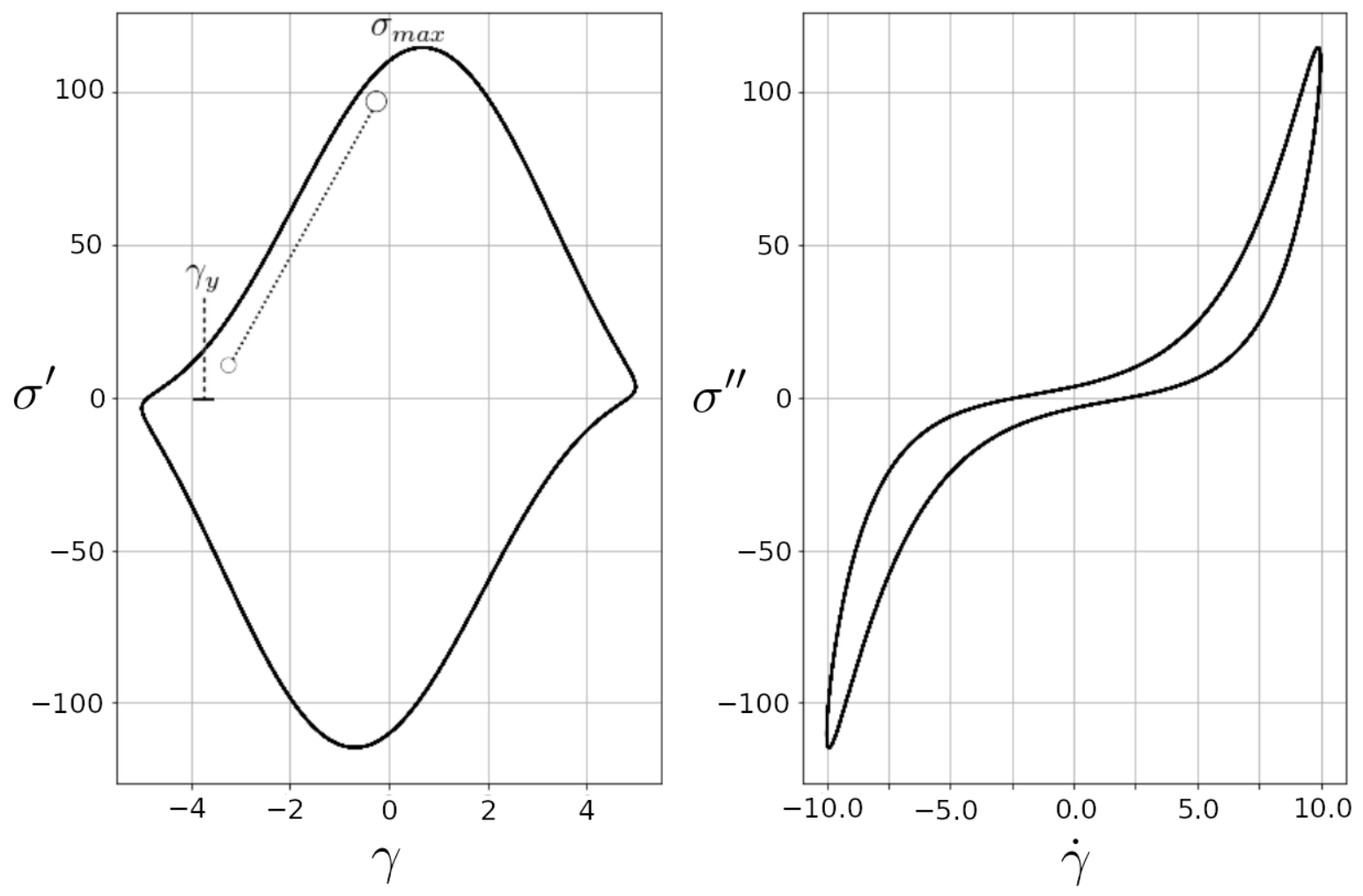
Figure 6.9: Plots of elastic $\left(\sigma^{\prime}[\mathrm{Pa}]\right)$ and viscous $\left(\sigma^{\prime \prime}[\mathrm{Pa}]\right)$ Lissajous-Bowditch curves as a function of $\gamma$ and $\dot{\gamma}\left[\mathrm{s}^{-1}\right]$ for $W i=2, D e=0.1, D e_{a}=1, \lambda=1, \beta=30, \xi=1$ and $\phi=0.3 \%$. The cage moduli is represented by $G_{r}$ and the maximum stress is $\sigma_{\max }$. [Source: the author.]
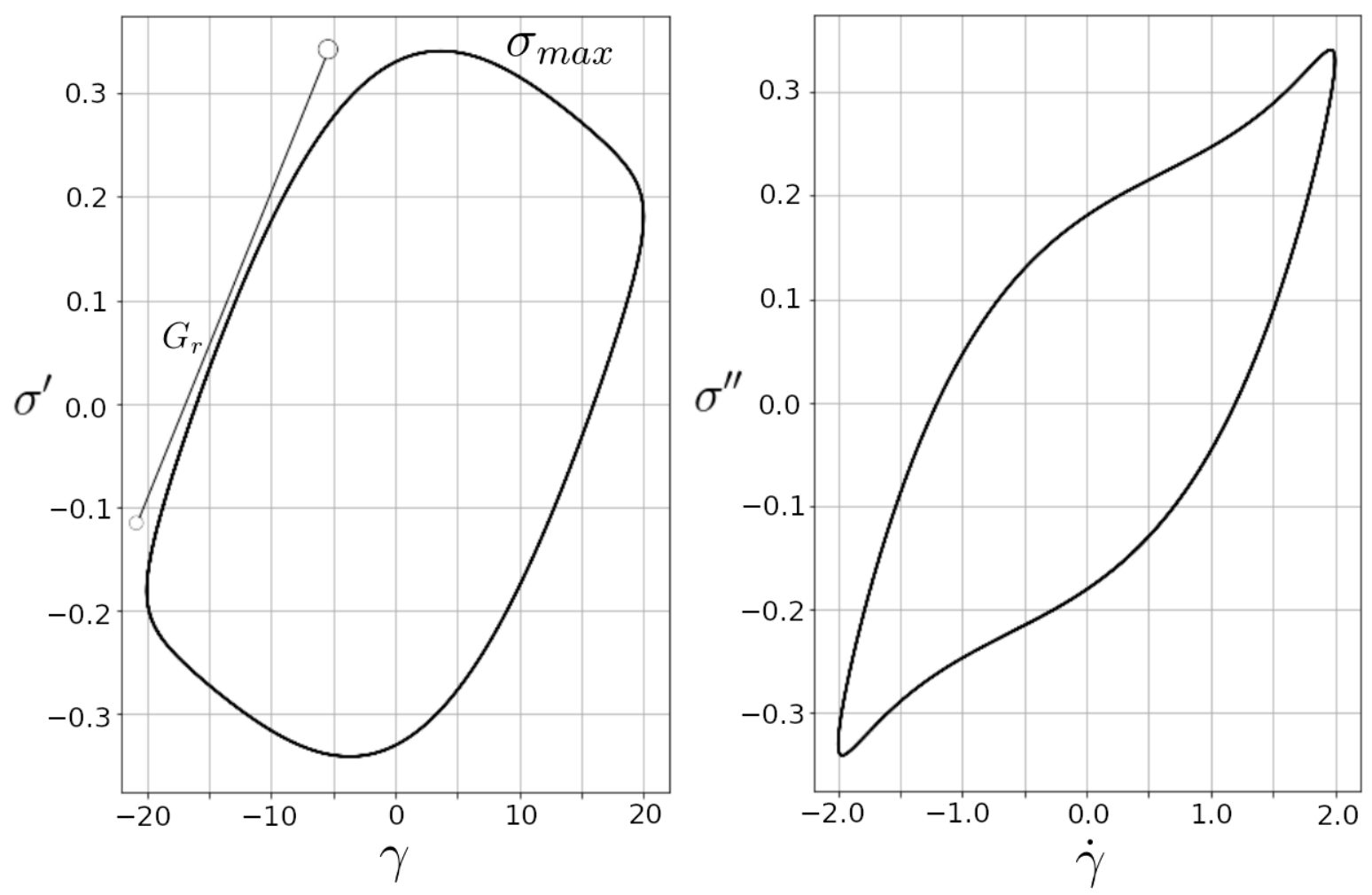


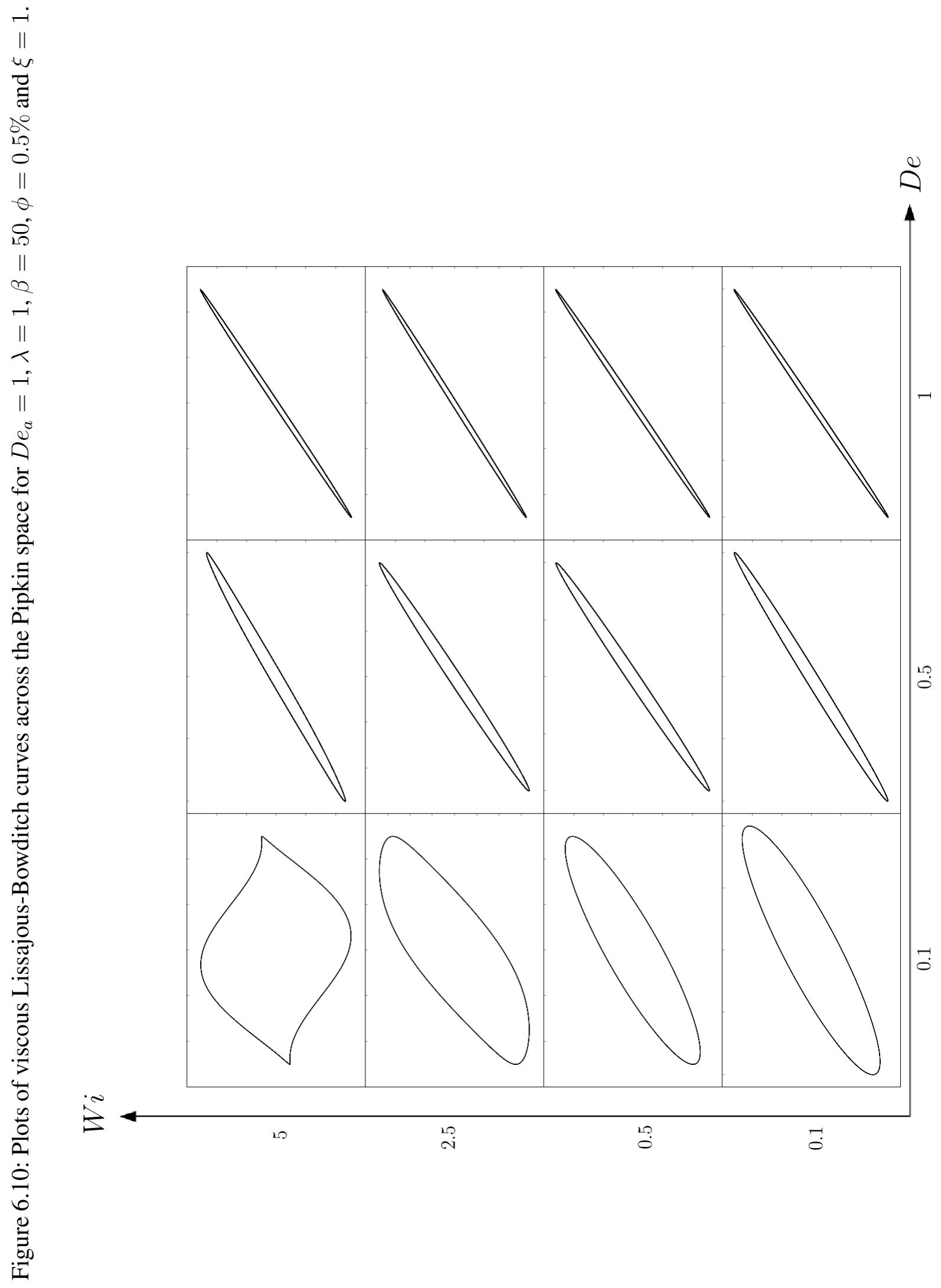




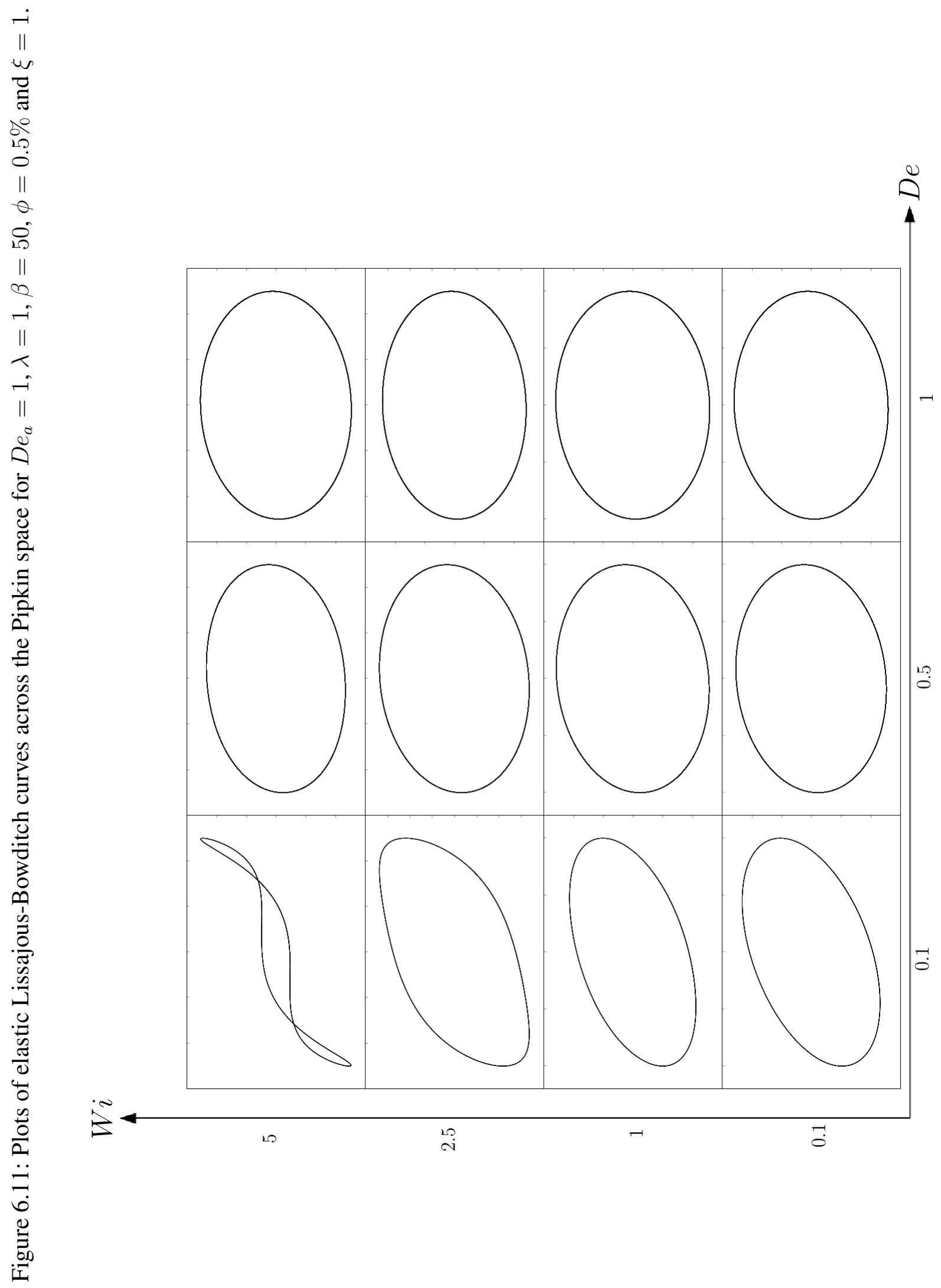




\subsection{Normal Stress Differences}

Usually, the stress tensor can be represented as the sum of hydrostatic pressure and the deviatoric component. If pressure is not high, it is possible to neglect the compressibility of liquid relevant in some real situations. Therefore, only the deviatoric part of the stress tensor is important for deformation of fluids. It means that if the hydrostatic pressure is superimposed, it will change all normal components of the stress tensor but will not influence flow. The direct consequence of this approach is that in order to characterize the effect of normal stresses in shear flow, it is not the absolute values of normal stresses but their differences that are important.

Usually, the normal stresses are smaller than the shear stresses at low shear rates but they grow along with an increase of the shear rate much faster than shear stresses. That happens because $N_{1}=\sigma_{11}-\sigma_{22}$ is a second order effect in relation to shear strain, $N_{1} \propto \gamma^{2}$. Furthermore, the first normal stress difference is the second order effect in the whole shear rate range, including the range of non-Newtonian flow.

The literature abounds with studies of either normal stress differences, but seldom are both discussed for a given material. Most of the studies on normal stress differences are conducted on polymeric systems (i.e. melts and solutions). The second normal stress difference, though not very large, is usually related to instabilities and development of the elastic-type secondary flows in the movement of liquid and fractures of viscoelastic liquids and suspensions (MALKIN AND ISAYEV, 2011). In our case, it is directly related to the destruction and formation of nematode's aggregates.

The first normal stress difference in large amplitude oscillatory shear consists of two contributions: one from non-oscillating nonzero mean value and the other from oscillating even harmonics, while the nonlinear shear stress can be expressed as a sum of odd harmonics (NAM et al., 2010). In polymer solutions or melts, the existence of normal stress differences can be attributed essentially to changes in molecular conformation, and perhaps alignment. In moderate shear environments, when chain scission does not occur, the individual molecules are elastically strained, and they recover their equilibrium conformation when the stress field is removed or dissipated.

As seen in figure 6.12, the Lissajous curve of normal stress versus shear strain for the suspensions we studied had a symmetrical, butterfly-like shape, and the area enclosed by the curve was 
related to the phase difference. Because of the left and right symmetry of the curve, the stress could be divided into three intervals according to the strain changes. Figure 6.12 shows the variation in structural strain as the normal stress changes. In the first interval, the structural strain increased with increasing shear strain, leading to a decrease in the normal stress. When the shear strain reached its maximum, the normal stress increased slightly due to the reduction of the shear rate and the reorganization of the chain-like structure. In this interval, the structural strain was large, the interparticle space increased, and the chain-like structure was destroyed. In the second interval, as the strain decreased, the structural strain decreased rapidly until it reversed. The normal stress increased and the nematodes formed a chain-likes structure. In the third interval, as the strain continued to increase, the chain-like structure was destroyed. The normal stress reduced with the increasing structural strain, causing the normal stress to decrease.

The phase angle behavior of the first normal stress difference can be easily confirmed through the Lissajous patterns in figure 6.13, as a function of the Deborah number. The characteristics of the materials are confirmed by the ellipsoidal shape of shear stress with respect to shear strain. Areas of the ellipsoid of the shear stress vs. shear strain are directly related to the loss modulus of the materials. The Lissajous patterns of the suspension is close to a line indicating that the shear stress is nearly in-phase with the shear strain. Furthermore, we observe that when the Deborah number is too small or too large, the difference in phase of normal stress and shear strain approaches $\pm \pi / 2$.

It is interesting to note the existence of secondary loops in these Lissajous-Bowditch curves. In normal stresses, they are related to the $2 \omega$ dependence of $N_{1}$, as seen in the frequency spectrum of figure 6.14. However, in shear stress, the interpretation of secondary loops has, to date, been limited to the study of specific material examples, being related to physical microstructural features such as non-affine deformation (JEYASEELAN AND GIACOMIN, 2008) and the absence of long-chain branching in polymer melts (STADLER et al., 2008). However, such secondary loops have been observed for many different material systems including micellar solutions (EWOLDT et al., 2008b), a polystyrene solution (JEYASEELAN AND GIACOMIN, 2008), several molten polymers (TEE AND DEALY, 1975), star-polymer networks as well as Xanthan gum solutions and an invert-emulsion drilling fluid. Nonlinear constitutive models can also show secondary loops, examples include a non-affine network model, a tube-based model of entangled linear polymers (LEYGUE et al., 2006) and a single mode Giesekus model (EwOLDT et al., 2008b).

In addition, using Gordon-Schowalter derivative, we may compute second normal stress difference, $N_{2}$, as seen in figure 6.14. If the slip parameter $\xi$ is set to zero, the normal stresses cannot 
be predicted by the model as depicted in figure 6.15. Suspensions of rigid non-colloidal particles are known to exhibit a strong negative second normal stress difference, in particular when the suspension is concentrated, giving rise to secondary flows (SIGINER, 2015). Since $N_{2}$ can also be seen as a measure of the relative stretching of particles in the direction of the velocity gradient vs. the neutral direction, its negative sign implies the nematode's elongation. The normal stress differences are deeply related to the nonlinear aspects of the solution. Variables such as shear rate, temperature, particle size and shape, volume fraction and interaction are related to these measurements. To sum up, normal stresses arise due to anisotropy and orientation of particles and a robust model should compute both $N_{1}$ and $N_{2}$.

\subsection{Chapter conclusions}

In this chapter, we proposed a constitutive model based on a flexible orientational filament. The model was tested with both only affine and also non-affine motion. That means that the local strain in a sample after deformation is not identical everywhere due to the concentration differences and the elastic disturbances caused by the active particle intrinsic motion. While the nematodes centers follow the macroscopic shear deformation affinely, the head and tail can deviate from the affine deformation field in order to minimize the global free energy.

The model was validated with experimental data in linear regime (OSAS) and independent analysis from nonlinear regime (LAOS). Using the Lissajoud-Bowditch approach, the Pipkin diagrams were constructed using different parameters. We observed that at sufficiently low Deborah number, the viscoelastic material has enough time to relax and behaves like a Newtonian liquid for small amplitudes. The material was driven into the nonlinear region, when $W i$ was increased to high values but $D e$ was kept constant.

The normal stress difference was also studied. It increased when the nematodes formed a chain-like structure, until it is destroyed. Furthermore, using Gordon-Schowalter derivative, we were able to compute the second normal stress difference, $N_{2}$. Its negative sign is related to the nematode's elongation during shear at large amplitudes.In the next chapter, we introduce the use of microrheometry to analyze active suspensions. 
Figure 6.12: The plot shows the Lissajous-Bowditch curve of the first normal stress difference, $N_{1}(t)[\mathrm{Pa}]$ and the shear strain with its plots as functions of time $[\mathrm{s}], N_{1}(t)$ and $\gamma(t)$. The parameters used were $W i=1, D e=0.5, D e_{a}=1, \lambda=1, \beta=50, \xi=1$ and $\phi=1 \%$. [Source: the author.]
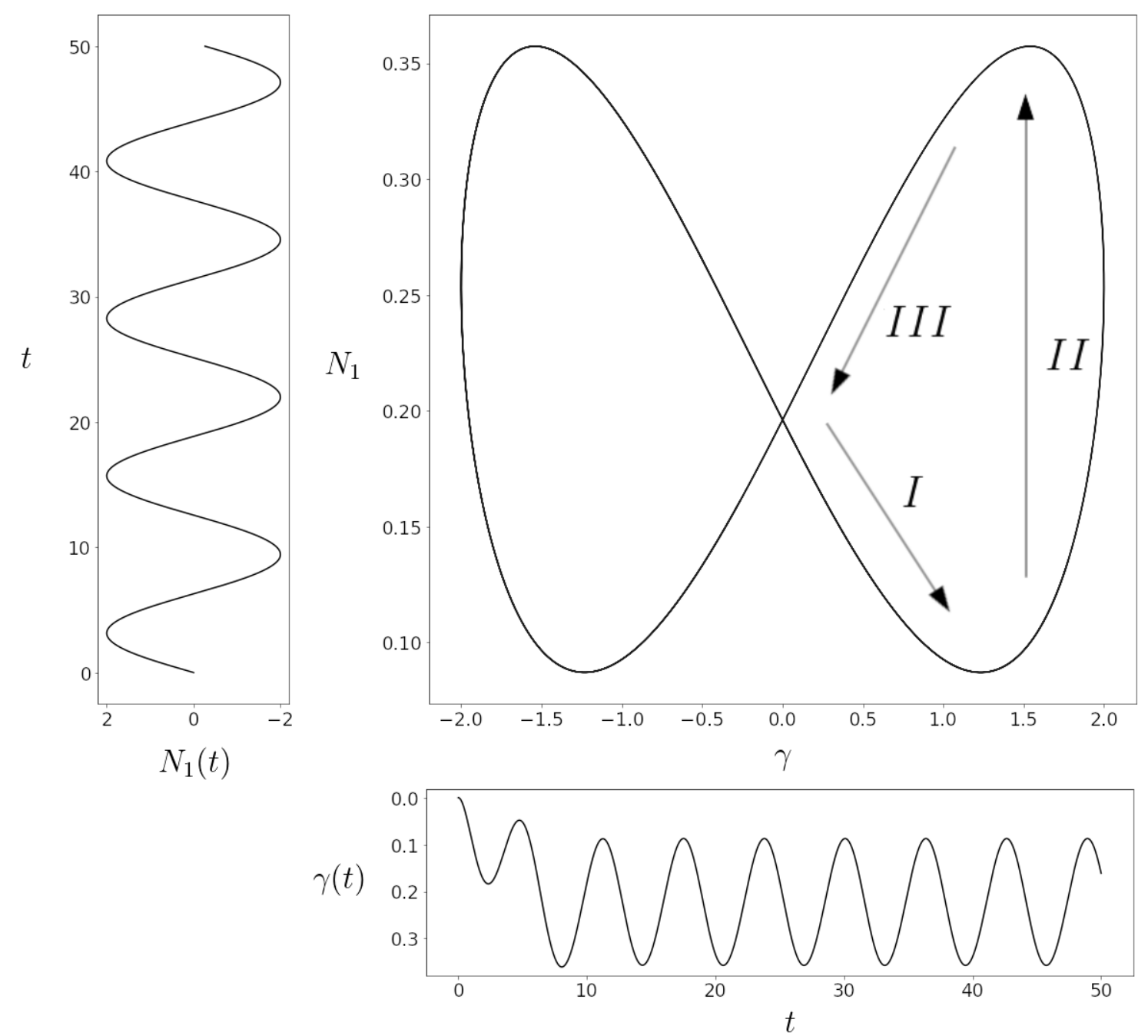


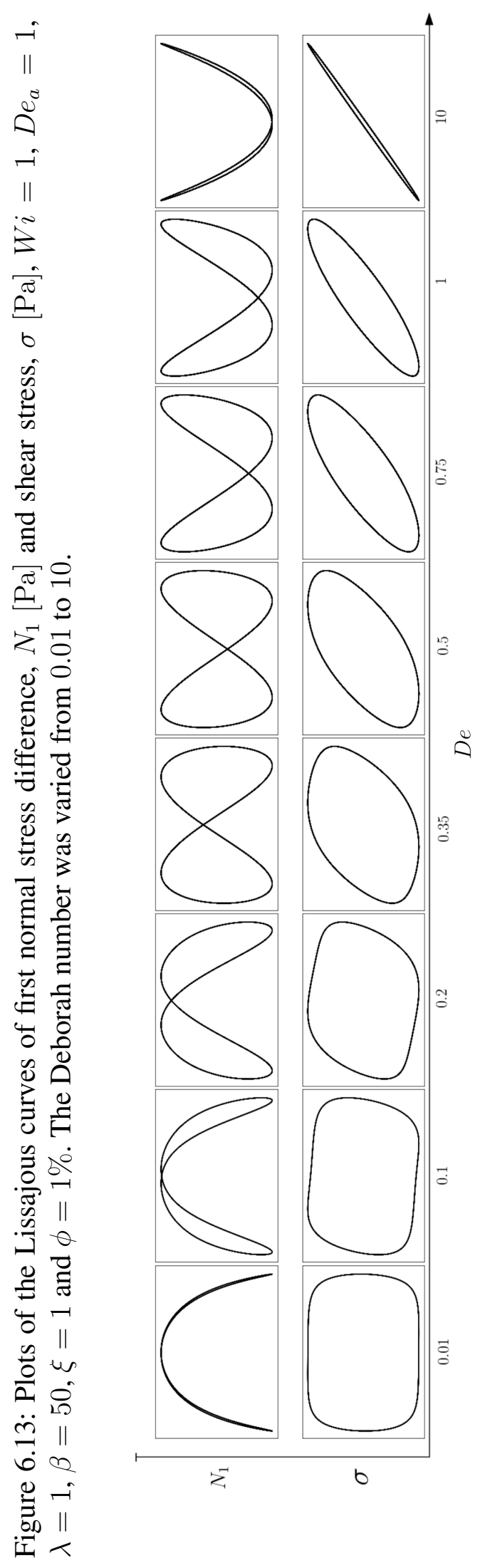


Figure 6.14: The first plot shows the shear stress $\sigma(t)[\mathrm{Pa}]$ (dashed line), first normal stress difference, $N_{1}(t)[\mathrm{Pa}]$ (solid line) and second normal stress difference, $N_{2}(t)$ [Pa] (black circles) as a function of time, $t \mathrm{~s}$, for $W i=1, D e=0.5, D e_{a}=1, \lambda=1, \beta=50, \xi=1$ and $\phi=1 \%$. The second plot presents the Fast Fourier Transform as a function of frequency [rad] of the shear stress (solid line) and the first normal stress difference (dashed line). [Source: the author.]
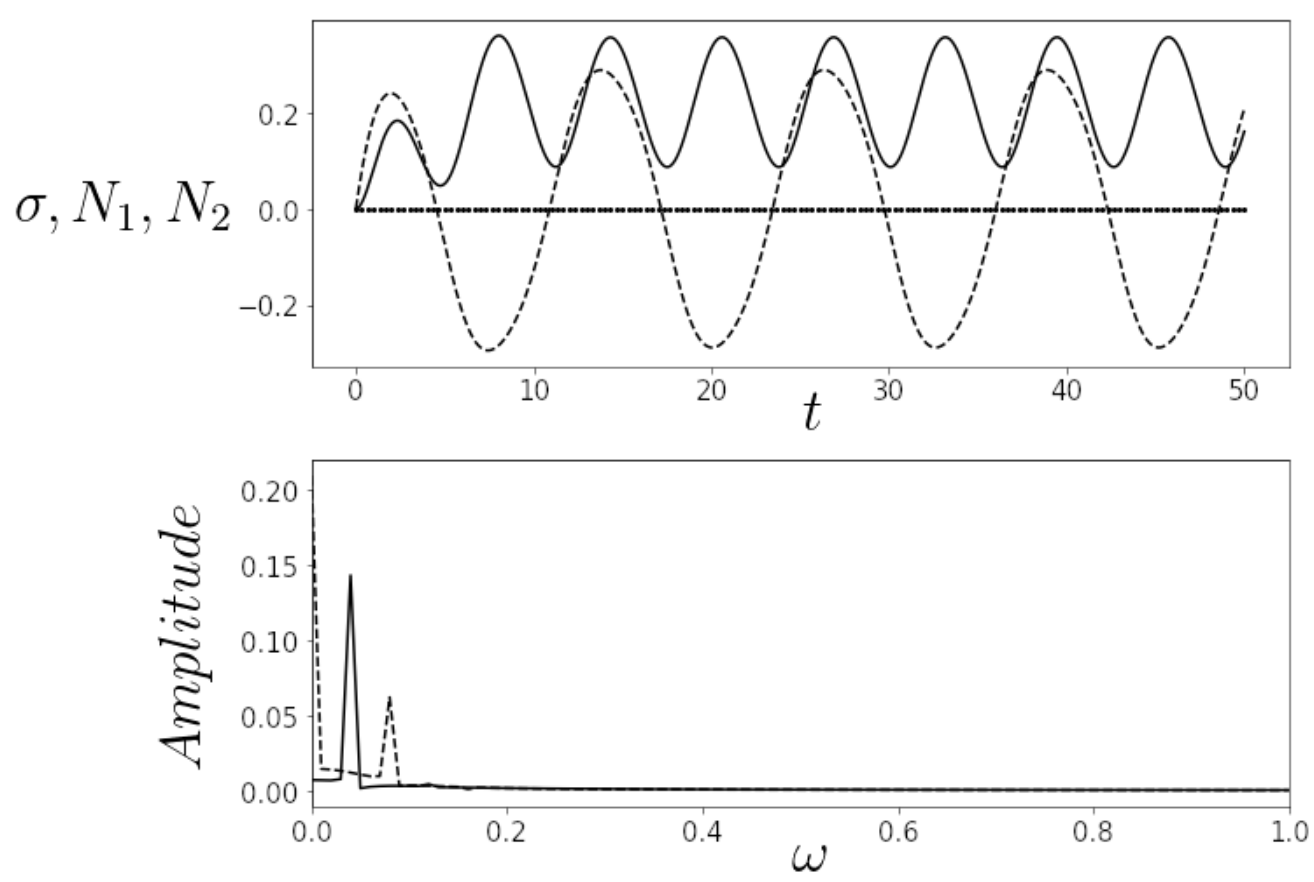
Figure 6.15: The plot shows the shear stress $\sigma(t)[\mathrm{Pa}]$ (solid) and the second normal stress difference $N_{2}(t)[\mathrm{Pa}]$ for $\xi=0$ (black circles) and $\xi=1$ (dashed line), as a function of time, $t \mathrm{~s}$. The parameters of the model are $W i=1, D e=0.5, D e_{a}=1, \lambda=1, \beta=50, \xi=1$ and $\phi=1 \%$. [Source: the author.]

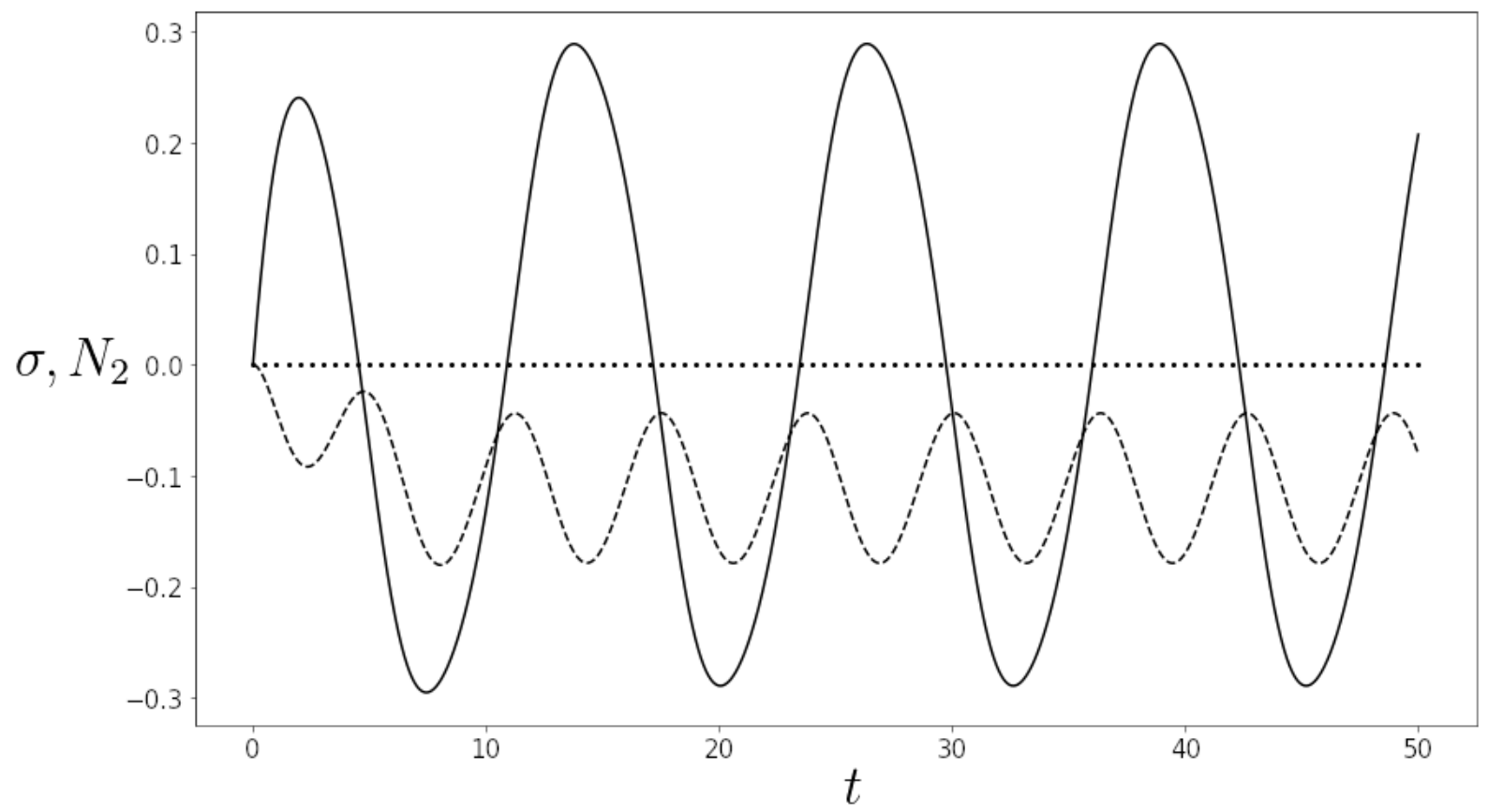




\section{Microrheometer}

In this chapter, a microrheometer developed at the University of Pennsylvania (Penn Complex Fluids Lab, directed by Professor Paulo Arratia). This new methodology is still under development. Thus, the experiments performed in this chapter were performed with polymer particles. However, the experimental and theoretical methodology could (and will be in future works) also be used for living particles. A pressure pump is coupled to a Poly(methyl methacrylate) (PMMA) microchannel with square cross-sectional area of $\sim 275 \mu \mathrm{m}$. The channel is observed with a microscope and the flow is recorded using a CCD camera. Pressure sensors (Wet/Wet Differential Pressure Transducer - model PXM409-025HDWUV from Omega) are used to obtain the correct applied pressure. The sensors are coupled to flexible microtubes in a $\mathrm{T}$ formation. One of the $\mathrm{T}$ inlets is associated with a pressure pump, while the other is directly connected to the microchannel. A schematic of the microchannel is presented in figure 7.1 and the microrheometer in figure 7.2. The microchannel was produced by $3 \mathrm{D}$ printing and sealed using a thin PMMA plate and Acetonitrile.

Figure 7.1: Microchannel schematic. [Source: the author]

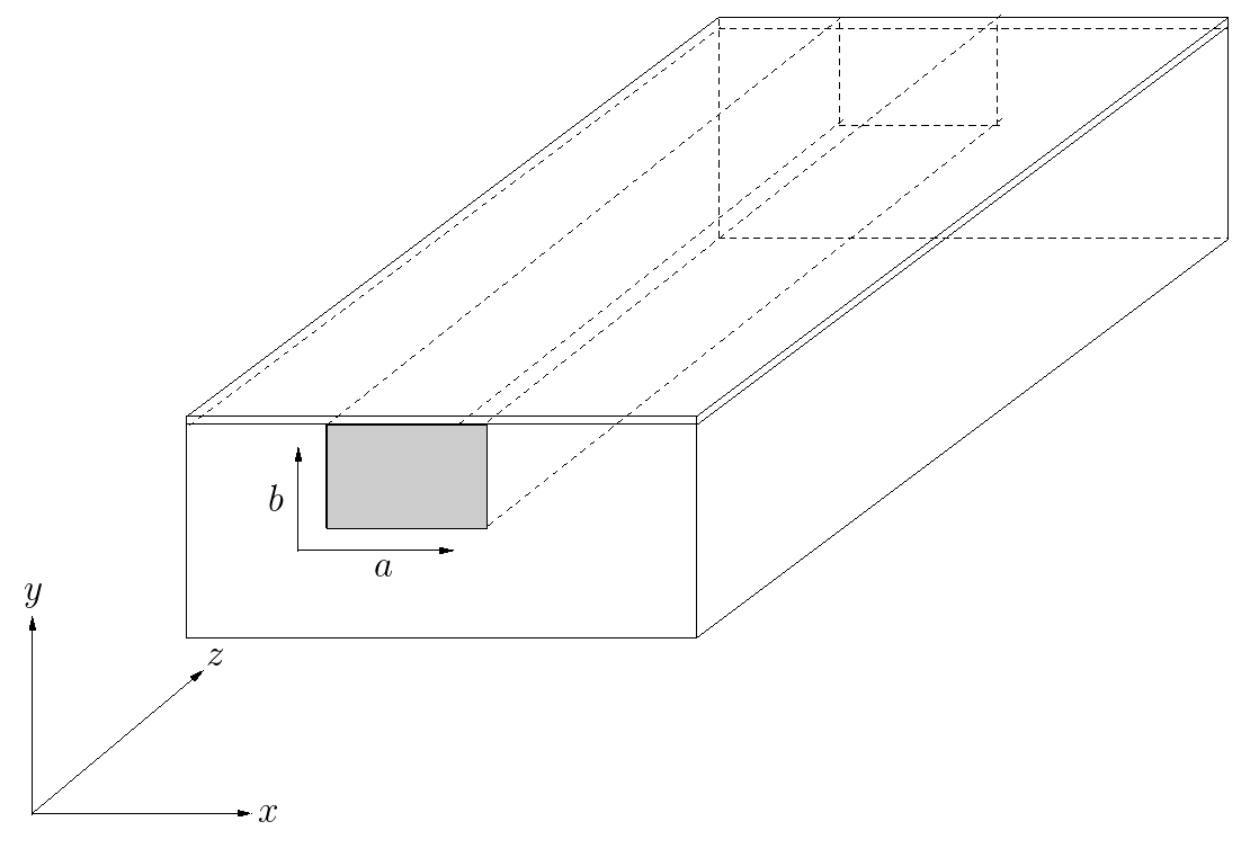

Classical macroscopic rheometry techniques for measuring fluid properties in shear and elongation typically involve characteristic length-scales $\mathcal{O}(1 \mathrm{~mm})$, require sample volumes $\mathcal{O}(1 \mathrm{~mL})$ 
Figure 7.2: Microrheometer schematic. [Source: the author]

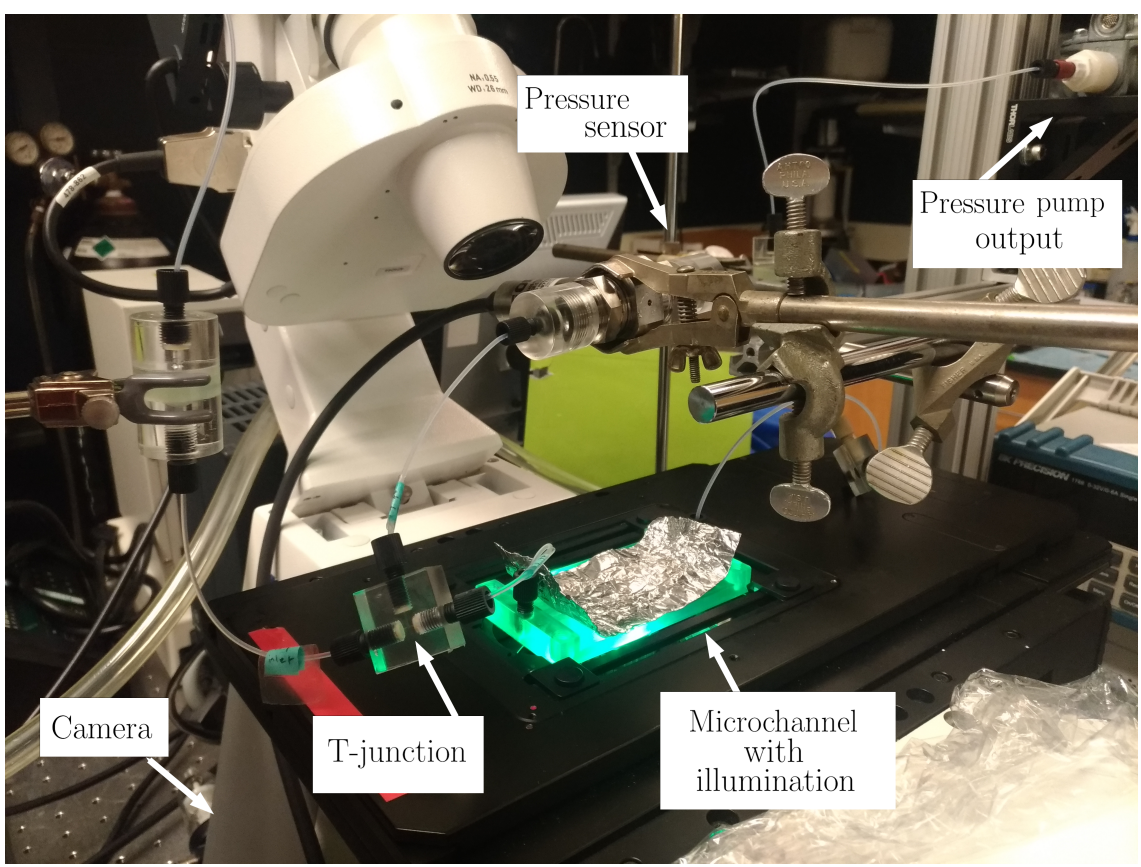

and probe deformation rates of perhaps up to $\mathcal{O}\left(100-1000 \mathrm{~s}^{-1}\right)$. While these methods are satisfactory for understanding the behaviour of many fluids in a wide variety of flows, there are circumstances when using devices with smaller length-scales to investigate rheological response may be advantageous.

It is immediately clear that the advantage of small sample volumes plays a particularly important role in biological systems. The amount of material (in particular proteins) is often very limited, and under these conditions it may not be feasible to produce enough for $1 \mathrm{~mL}$ of solution. In this case, microrheology, a technique used to measure the rheological properties of a medium, such as microviscosity, via the measurement of the trajectory of a flow tracer (a micrometre-sized particle), can be used. The motion of the tracer particles reflects the rheological properties of their local environment. In usual rheometers, errors in torque and angular deflection measurement as well as in determining the true sample geometry influence the resulting viscosity calculations. In this condition, it is very difficult to measure low viscous fluids due to the torque resolution. However, it is not a problem in microrheology and it is possible to obtain very accurate results.

The microrheometer of University of Pennsylvania, uses video-particle tracking to make the rheological measurements. This method relies on the motion of a tracer particle within a material 
that needs to be characterized. Video tracking allows measuring the compliance and can yield a complete characterization of the linear viscoelasticity of the matrix. The most challenging aspects of a video tracking experiment are often the process of acquiring the trajectory of a number of particles and the image analysis, which consists in analyzing individual frames to extract the coordinates of all the particles in the frame and matching the particles through subsequent frames to produce data of trajectories.

In this case, the microrheometer consists in a thin microchannel of $275 \mu \mathrm{m}$ width and $9.05 \mathrm{~cm}$ long. A pressure driven flow is applied at the inlet of the channel and fluorescent particles (Thermo Scientific ${ }^{T M}$ Fluoro-Max Dyed Green Aqueous Fluorescent Particles) of polystyrene with $2 \mu \mathrm{m}$ are added to the flow in order to obtain the velocity profile.

\subsection{Particle tracking analysis}

The video analysis enables us to extract the trajectories of individual microspheres from a video of their microscope images. The time evolution of the distribution of particles, namely

$$
\rho(\boldsymbol{r}, t)=\sum_{i=1}^{N} \delta\left(\boldsymbol{r}-\boldsymbol{r}_{i}(t)\right)
$$

then can be used to calculate quantities of interest. In equation 7.1, $\boldsymbol{r}(t)$ is the location of the $i$-th particle in a field of $N$ particles at time $t$ and $\rho(\boldsymbol{r}, t)$ is the trajectory.

The software based on IDL, a programming language optimized for visual data analysis, to extract any particle information $\rho(\boldsymbol{r}, t)$ from a sequence of digital images, consists of five logical steps: correcting imperfections in the individual images, locating candidate particle positions, refining these positions, discriminating "false" particles, and finally linking the time-resolved particle locations into trajectories.

The difficulty of measuring $\rho(\boldsymbol{r}, t)$ can vary greatly from system to system. For instance, images of a dilute suspension whose particles are geometrically confined at the microscope's focal plane are simpler to process than pictures of a dense suspension of colloid moving in three dimen- 
sions. That is one of the reasons why we are using a dilute suspension to analyze the particles.

Digitized images typically suffer from a range of imperfections including noise, nonuniform contrast and geometric distortion. These all introduce errors into $\rho(\boldsymbol{r}, t)$ unless steps are taken to restore the image to its "ideal" state. Some geometric distortions are caused by defects in the microscope optics, but most are introduced in later stages of digitization.

Contrast gradients can arise from nonuniform sensitivity among the camera's pixels. Significant variation is often due to uneven illumination. Usually, the background is then subtracted, but it may be difficult if the features of interest are relatively small and diluted as is frequently the case for colloidal images. Under these circumstances, the background is reasonably well modeled by a boxcar, $A_{w}$, average over a region of extent $2 w+1$, where $w$ is an integer larger than a single sphere's apparent radius in pixels, but smaller than the intersphere separation:

$$
A_{w}(x, y)=\frac{1}{(2 w+1)^{2}} \sum_{i, j=-w}^{w} A(x+i, y+j)
$$

Another problem that actually destroys information is noise. Some types of noise, such as that from CCD camera are unavoidable. They tend to be purely random with a correlation length $\lambda_{n} \approx 1$ pixel. Convolving an image $A_{w}(x, y)$ with a Gaussian surface of revolution of half width $\lambda_{n}$ strongly suppresses such noise without unduly blurring the image (CROCKER AND GRIER, 1996):

$$
A_{\lambda_{n}}(x, y)=\frac{1}{B} \sum_{i, j=-w}^{w} A(x+i, y+j) \exp \left(-\frac{i^{2}+j^{2}}{4 \lambda_{n}^{2}}\right)
$$

with normalization $B=\left[\sum_{i, j=-w}^{w} \exp \left(-\frac{i^{2}+j^{2}}{4 \lambda_{n}^{2}}\right)\right]^{2}$. The difference between the noise-reduced and background images is an estimate of the ideal image. Using a convolution kernel, we can compute equations 7.2 and 7.3 .

The particles are then identified within an image as local brightness maxima. In practice, a pixel is adopted as a candidate if no other pixel within a distance $w$ is brighter. Having already found a locally brightest pixel at $(x, y)$, which presumably is near a sphere's geometric center at 
$\left(x_{0}, y_{0}\right)$, we calculate the offset from $(x, y)$ to the brightness-weighted centroid of the pixels in a region around $(x, y)$ :

$$
\left(\begin{array}{c}
\epsilon_{x} \\
\epsilon_{y}
\end{array}\right)=\frac{1}{m_{0}} \sum_{i^{2}+j^{2} \leq w^{2}}\left(\begin{array}{l}
i \\
j
\end{array}\right) A(x+i, y+j),
$$

where $m_{0}$ is the integrated brightness of the sphere's image. The refined location estimate is then $\left(x_{0}, y_{0}\right)=\left(x+\epsilon_{x}, y+\epsilon_{y}\right)$. The background subtraction performed by the convolution kernel in equations 7.2 and 7.3 avoids biasing $\epsilon_{x}$ and $\epsilon_{y}$ toward the center of the fitting region and away from the particle image's centroid. If neither $\left|\epsilon_{x}\right|$ nor $\left|\epsilon_{y}\right|$ exceeds 0.5 , the candidate centroid location can be moved accordingly and the refinement recalculated.

After determining the location of each particle, we can determine which particle in a given image most likely corresponds to one in the preceding image. Tracking more than one particle requires care since any particle can be identified with only one particle in each of the successive and preceding frames. Thus, we seek the most probable set of $N$ identifications between $N$ locations in two consecutive images. If the particles are indistinguishable, as for monodisperse colloidal spheres, this likelihood can be estimated only by proximity in the two images. More information regarding the probabilistic theory involved in this step can be found in (CROCKER AND GRIER, 1996), The implementation of this method is also available in Python as Trackpy (VAN DER WEL et al., 2010). An example of the particles already identified is seen in figure 7.3.

\subsection{Newtonian and polymeric suspensions}

As a first approach, a $75 \%$ glycerol aqueous solution was used as the working fluid. One drop of fluorescent particles (Thermo Scientific ${ }^{T M}$ Fluoro-Max Dyed Green Aqueous Fluorescent Particles) of polystyrene with $2 \mu \mathrm{m}$ were added in order to obtain the velocity profile for each $\mathrm{mL}$ of solution. An oscillatory signal with DC component was applied using the MFCS-EZ (Fluigent) pressure pump with frequency of approximately $\omega=1 \mathrm{rad} \mathrm{s}^{-1}$.

The small length-scales characteristic of microfluidic devices generally result in flows of liquids in which viscous stresses dominate inertia with typical Reynolds numbers $R e=\rho U d / \eta<$ 
Figure 7.3: Identified particles in water. The particles with especially low mass or especially large size are probably out of focus or aggregated, respectively.

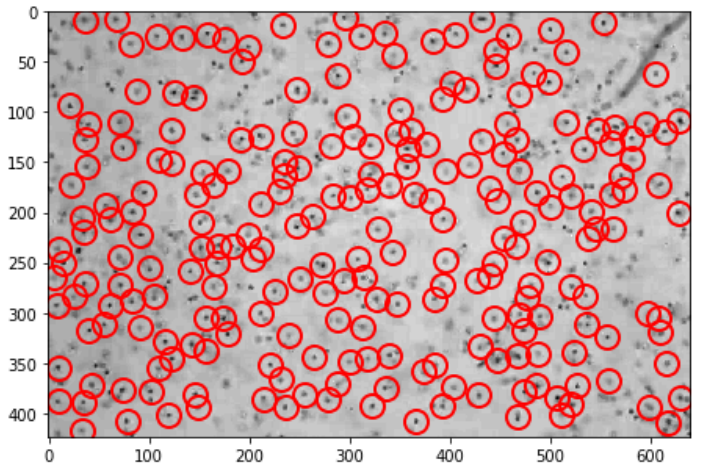

1, where $\eta$ is the dynamic viscosity, $\rho$ is the fluid density, $d$ is a characteristic time scale (such as the particle diameter) and $U$ is a characteristic velocity of the flow which is controlled by the volumetric flow rate $U \sim Q / d^{2}$. While low Reynolds number flows present challenges that limit efficient mixing in microfluidic systems, they are advantageous when seeking to impose laminar viscometric flows with controlled kinematics and, for Newtonian fluids at least, it is possible to accurately measure steady two-dimensional and three-dimensional flows. For steady fully-developed flow in a rectilinear channel the pressure drop is given by the force balance $w_{c} d \Delta P=2 L\left(w_{c}+d\right) \tau$, where $L$ is the channel length. Hence the measured pressure drop $\Delta P=2 \tau L\left(w_{c}+d\right) /(w d) \sim 2 \tau L / d$, can be very large when $L / d \gg 1$ and $w_{c} / d \gg 1$.

The shear rate, an important variable for estimating polymer deformation, is defined in the microchannel as

$$
\dot{\gamma}=\frac{\partial U_{z}(x, y)}{\partial x}
$$

where $U_{z}(x, y)$ is the velocity profile in the $z$ direction, given by (POZRIKIDIS, 2011) 


$$
U_{x}(y, z)=\frac{1}{2 \eta}\left(\frac{d P}{d z}\right)\left(y^{2}-b^{2}+d^{2} \sum_{n=0}^{\infty} 4(-1)^{n} \frac{\cosh \left(2 \alpha_{n} x\right) \cos \left(2 \alpha_{n} y\right)}{b \alpha_{n}^{3} \cosh \left(\alpha_{n} a\right)}\right)
$$

where $\alpha_{n}=(n+1 / 2) \pi / b, d P / d z$ is the pressure gradient in the streamwise direction, $\eta$ is the viscosity and $b$ is the height of the channel. In addition the shear stress, $\sigma_{y x}(y)$ is defines as

$$
\sigma_{x y}\left(y x=\frac{\Delta P}{L}\left(x-\frac{b}{2}\right) .\right.
$$

Based on that, the input pressure, which is directly related to the input stress, was measured using a Wet/Wet Differential Pressure Transducer (model PXM409-025HDWUV from Omega). The resulting signal is seen in figure 7.4. This signal will be the input of the LAOS stress test and it is normalized by the average pressure. It is important to filter the signal in order to avoid oversampling and unwanted noise.

Figure 7.4: Normalized input pressure $p / \bar{p}$ as a function of time, $t[\mathrm{~s}]$. The dashed signal represents the signal obtained from the pressure sensor. The solid line shows the filtered signal using a butterworth low-pass filter. [Source: the author.]

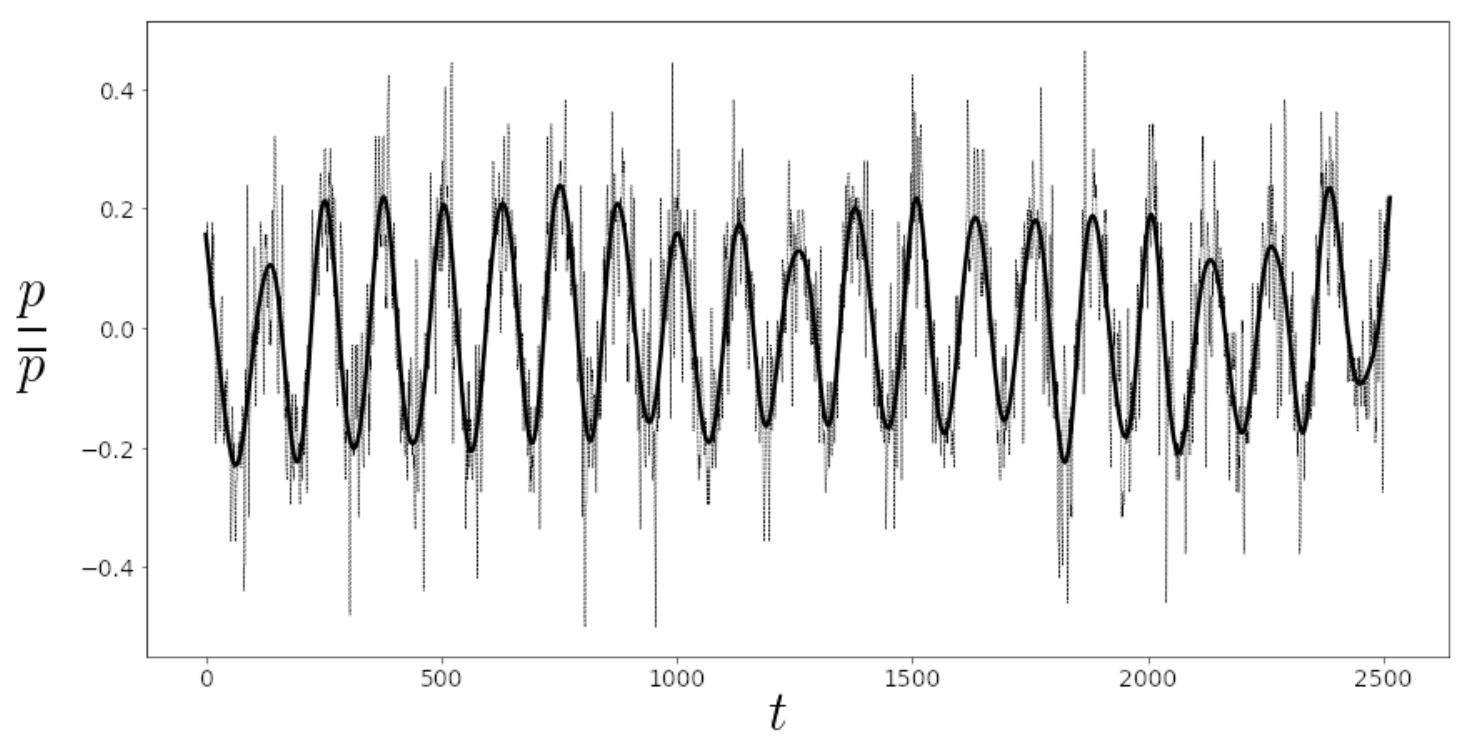

After recording the suspension motion using a Fastcam SA1.1 (from Photron) attached to a 
Observer.Z1 microscope (from Zeiss) at $60 \mathrm{fps}$, we were able to obtain the velocity of the suspension over time using the algorithm presented in the first section of this chapter. Next, we computed the position of each particle on each frame and obtained their trajectories. Figure 7.5 shows the time history of the velocity and pressure signal after the application of a butterworth low-pass filter. The velocity was also normalized by the average.

Figure 7.5: Normalized output velocity $v / \bar{v}$ and input pressure as a function of time, $t[\mathrm{~s}]$. The dashed signal represents the filtered signal. The velocity here is related to the strain-rate while the pressure is considered the input stress. [Source: the author.]
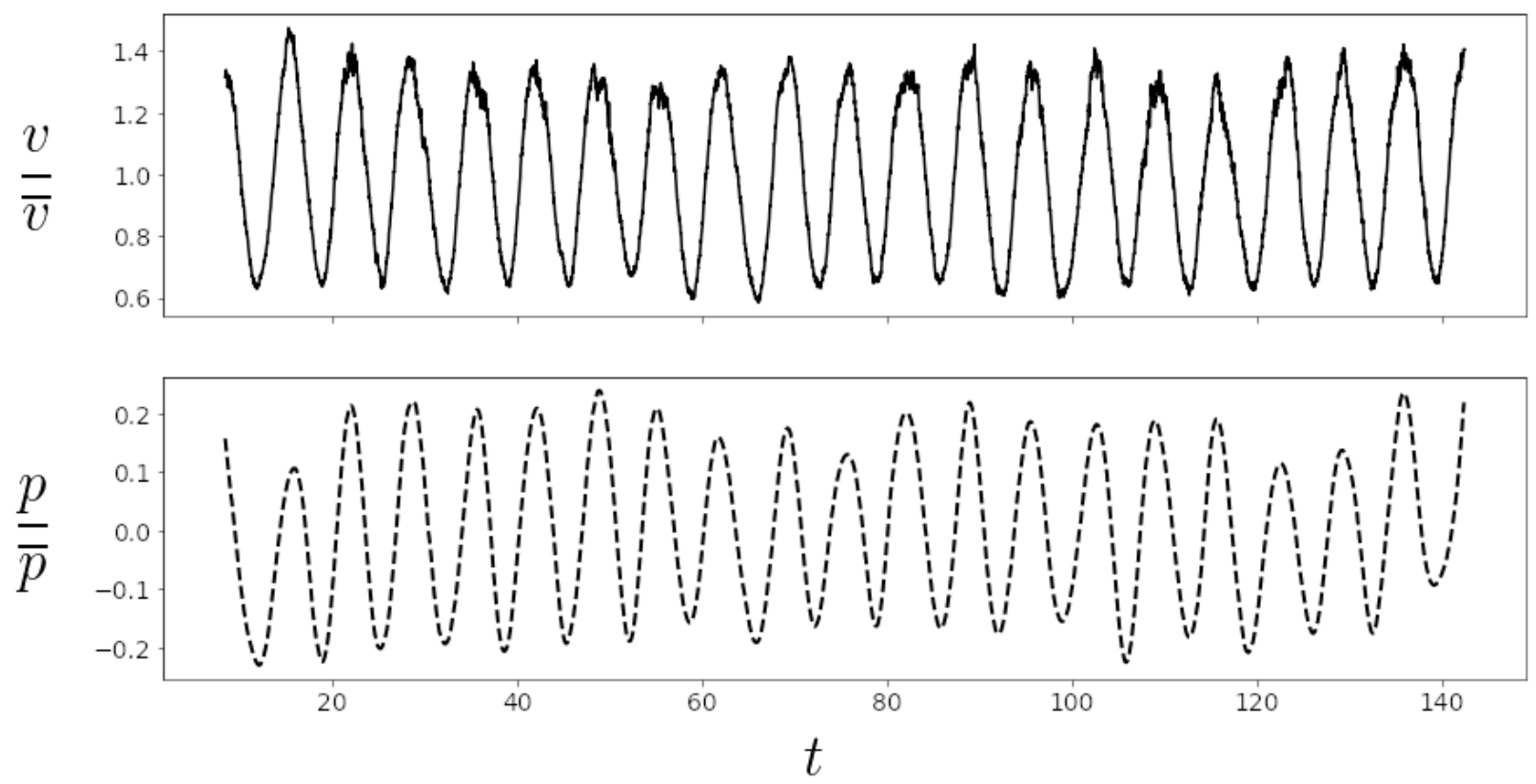

After testing the Newtonian solution, a suspension with $175 \mathrm{ppm}$ of poly(acrylic acid) with a molecular weight of 18 million in a $90 \%$ glycerol solution was used. Again, the input pressure was measured over time as presented in figure 7.6. The resultant velocity of the suspension over time is shown in figure 7.7 .

With both velocity and pressure (representing strain-rate and stress) we are able to compute the velocity profiles and the shear-rate as a function of the position. The velocity profile is calculated using one period of the root mean square (RMS) velocity signal. In figure 7.8 we can se a comparison between the theoretical velocity profile for a Newtonian fluid and the experimental re- 
Figure 7.6: Normalized input pressure $p / \bar{p}$ as a function of time, $t[\mathrm{~s}]$, for the non-Newtonian suspension. The dashed signal represents the filtered signal. [Source: the author.]

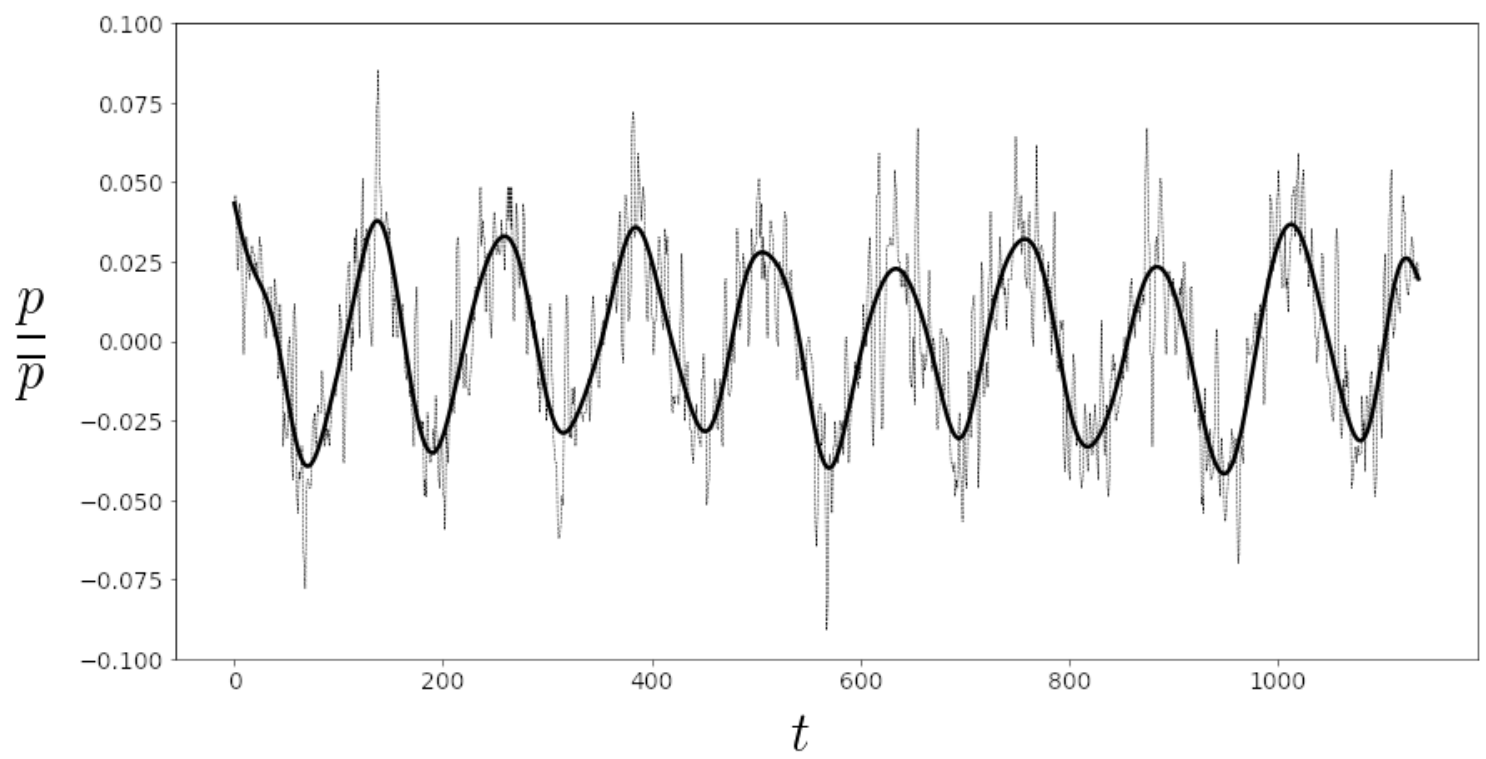

sult, showing excellent agreement. Figure 7.9 shows the velocity profile and shear-rate (computed by centered finite differences) for both Newtonian and non-Newtonian suspensions. It is important to highlight that approximatey $9 \%$ is removed from the proximity of the wall to errors. Thus, the distance $x$ in the case of the velocity profile extends from $x \simeq 12.5$ to $x \simeq 262.5$. In the case of shear rate, this distance removed is approximately $14 \%$ and the profile extends from $x \simeq 37.5$ to $x \simeq 237.5$.

As expected the velocity profile of the polymeric suspension has a small maximum value, due to the higher viscosity. In addition, the profile is less parabolic, showing a small shear-thinning behavior. The shear rate near the wall is also higher and more inclined for the non-Newtonian suspension. 
Figure 7.7: Normalized output velocity $v / \bar{v}$ and input pressure $p / \bar{p}$ as a function of time, $t[\mathrm{~s}]$, for the non-Newtonian suspension. The dashed signal represents the filtered signal. [Source: the author.]
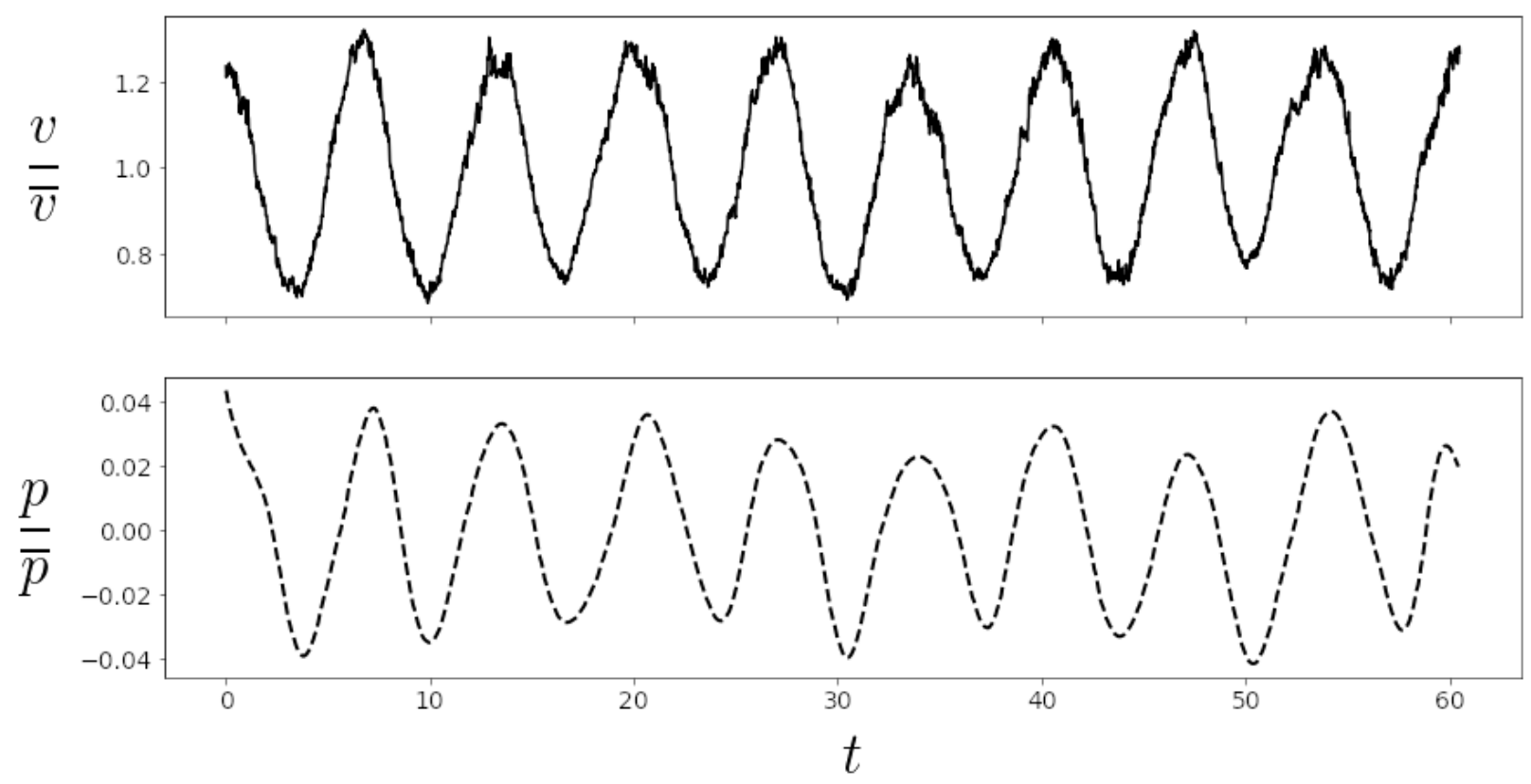
Figure 7.8: Comparison between theoretical (solid line) and experimental (black circles) velocity pofiles for Newtonian fluid.[Source: the author.]

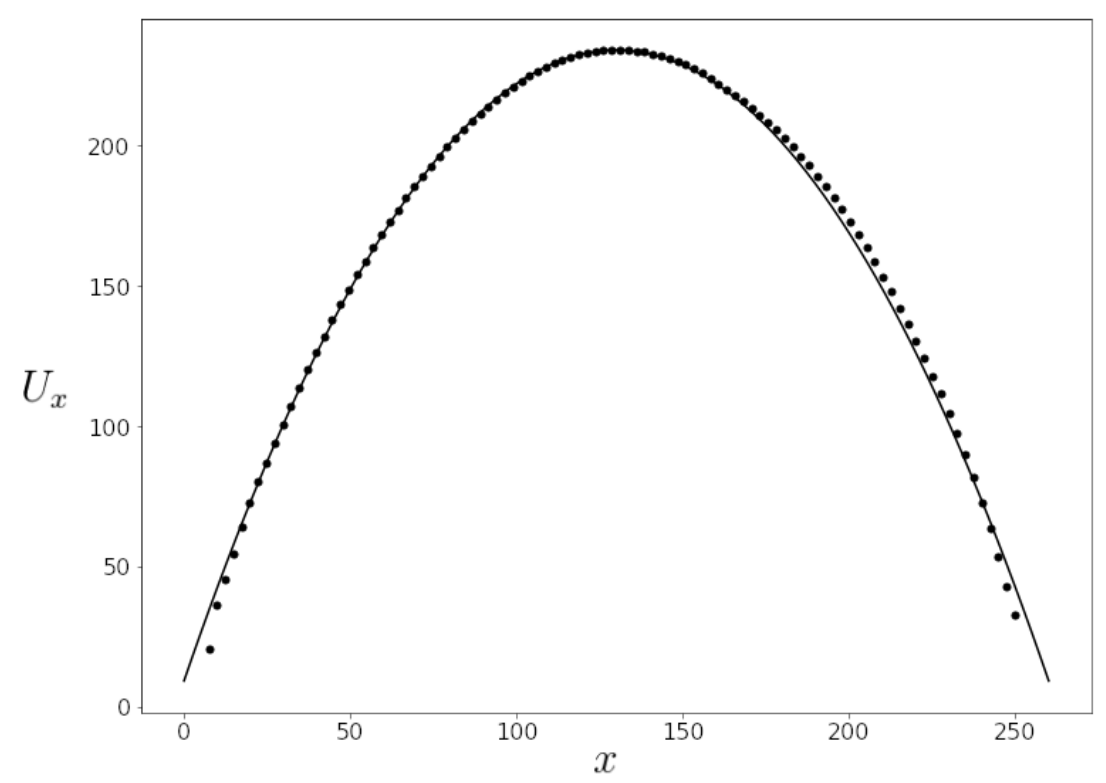


Figure 7.9: The left plot shows the velocity $[\mu \mathrm{m} / \mathrm{s}]$ profile obtained using the RMS velocity for the Newtonian (solid line) and non-Newtonian suspensions (dashed line). The right plot represents the shear-rate $\left[\mathrm{s}^{-1}\right]$ of the Newtonian (solid line) and non-Newtonian suspensions (dashed line) as a function of the position in the channel, $x \mu \mathrm{m}$. [Source: the author.]
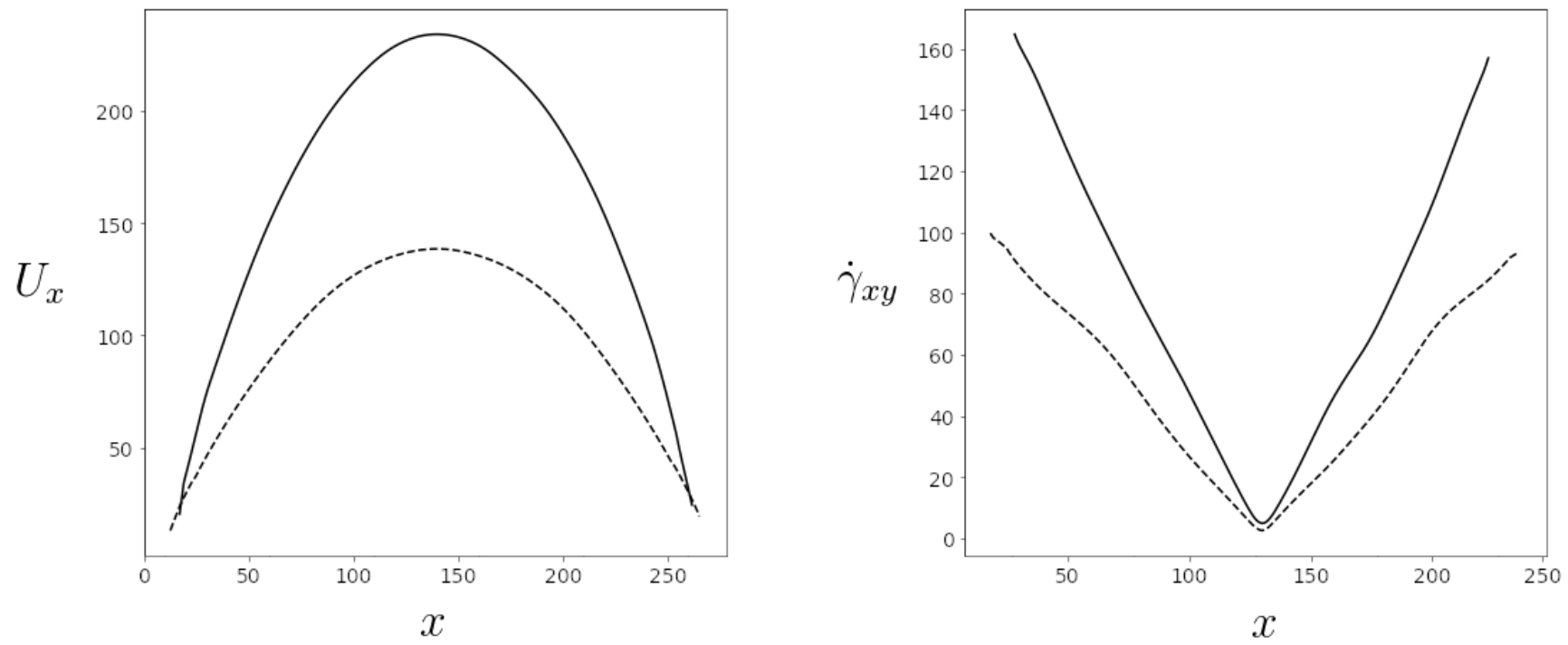


\subsection{Chebyshev expansion for UD-LAOS}

In chapter 2, the Chebyshev decomposition was presented for LAOS tests. Considering that UD-LAOS will also provide nonlinear signals, any orthogonal decomposition can be used to analyse the signals. Traditional small signal analysis uses Taylor expansion. However, this approach leads to large errors, or even divergence, when the input signal amplitude is large. Chebyshev series not only solves the convergence issues associated with Taylor expansions, but also results in much smaller approximation errors.

Volterra analysis involves the expansion of all system's nonlinearities in polynomial series (SARKAS et al., 2008). Traditionally, Taylor series is used. However, the convergence domain of a Taylor series is a disk in the complex plane (WAMBACQ AND S ANSEN, 1998). A complex singularity of the function being expanded reduces the convergence radius affecting the overall convergence for real values. The second drawback of Taylor series is their non-uniform error distribution behavior. The approximation error is very small near the expansion point but increases rapidly as the distance from the expansion point increases. This causes large errors when analyzing systems with large input signals.

On the other hand, the convergence domain of Chebyshev series is an ellipse (with semi axis $a$ and $b$ ) in the complex plain and has guaranteed convergence inside the interval $[a, b]$, as long as no singularities lie within this interval. This implies that complex singularities do not affect the convergence of the series, hence it converges for all nonlinearity functions found in practice (ISAACSON AND KELLER, 1994). Moreover, the min-max sense of the approximation results to equidistributed error within the interval $[a, b]$. This solves the error problem with large input signals.

Thus, a nonlinear function $f\left(\sigma^{\prime}\right)=f\left(\sigma+\sigma_{D C}\right)$, where $\sigma^{\prime}$ is the total stress which can be decomposed into

$$
f\left(\sigma+\sigma_{D C}\right) \simeq a_{0}+a_{1} \sigma+a_{2} \sigma^{2}+a_{3} \sigma^{3}+\ldots
$$

Where $\sigma_{D C}$ is the bias (or constant) stress and is considered known and $\sigma$ is te perturbation stress. In oscillatory shear, $\sigma$ is a sinusoidal excitation with amplitude $\gamma_{0}$, the serie needs to be calculated 
in the interval $\left[-\gamma_{0}, \gamma_{0}\right]$. The change of variables $\sigma=\gamma_{0} x$ is made. The Chebyshev coefficients $c_{n}$ of each $T_{n}(x)$ polynomial are calculated using Chebyshev quadrature as

$$
c_{n}=\frac{2}{\pi} \int_{-1}^{+1} \frac{f(\sigma+\gamma x) T_{n}(x)}{\sqrt{1-x^{2}}} d x .
$$

Thus, the truncated Chebyshev series of order $n$ is given by:

$$
S_{n}(x)=\frac{c_{0}}{2}+c_{1} T_{1}(x)+c_{2} T_{2}(x)+\ldots+c_{n} T_{n}(x)
$$

The reverse variable change $x=\sigma / \gamma_{0}$ is performed in the above equation and the Chebyshev polynomials are expanded, thus obtaining the final expression. The coefficients $a_{n}$ are linear combinations of the coefficients $c_{n}$. This comes from the fact that the polynomial $T_{n}(x)$ comprises monomials of orders $n, n-2, \ldots$. In other words, it is not possible to separe the DC effect from other harmonics. Equation 7.11 shows the relationship between $a_{n}$ and $c_{n}$ up to the fifth order, up to the fifth order, in which it is possible to identify almost all non-linearities of the signals (EwOLDT et al., 2008b).

$$
\begin{array}{r}
a_{0}=\frac{c_{0}}{2}-c_{2}+c_{4} \\
a_{1}=\frac{c_{1}}{\gamma}-\frac{3 c_{3}}{\gamma}+\frac{5 c_{5}}{\gamma} \\
a_{2}=\frac{2 c_{2}}{\gamma^{2}}-\frac{8 c_{4}}{\gamma^{2}} \\
a_{3}=\frac{4 c_{3}}{\gamma^{3}}-\frac{20 c_{5}}{\gamma^{3}} \\
a_{4}=\frac{8 c_{4}}{\gamma^{4}} \\
a_{5}=\frac{16 c_{5}}{\gamma^{5}}
\end{array}
$$




\subsection{Neural networks}

As mentioned in chapters 2 and 6, UD-LAOS is an interesting method to analyze how the structure of the fluid evolves during shear. However, no physical insight is obtained based on the typical rheological analysis such as Chebyshev polynomials, stress decomposition and Fourier transform analysis. As we demonstrated in the previous section, the DC component of strain (or stress) spread through all coefficients and the symmetry is broken. On the other hand, UD-LAOS is extremely important for the study of orientational particles. In a flow such as the unidirectional oscillatory, we can observe how the particles behave to oscillation only, without the reversible flow behavior. For that reason, we propose a completely new methodology, not yet present in the literature, to analyze the results obtained in UD-LAOS.

The methodology is based on machine learning. In a simple way, the idea is to remove the DC signal from the results obtained experimentally in UD-LAOS. We used a deep, artificial neural network known as multilayer perceptron (MLP) (SIMON, 1998). It is composed of an input layer to receive the signal, an output layer that makes a decision or prediction about the input, and in between those two, an arbitrary number of hidden layers that are the true computational engine of the MLP. MLPs with one hidden layer are capable of approximating any continuous function. Multilayer perceptrons are often applied to supervised learning problems: they train on a set of input-output pairs and learn to model the correlation (or dependencies) between those inputs and outputs. Training involves adjusting the parameters, or the weights and biases, of the model in order to minimize error. The algorithm of the densely-connected neural network layers was written using Python with Keras and Tensor Flow.

The building block for these neural networks are artificial neurons as shown in figure 7.10. These are simple computational units that have weighted input signals and produce an output signal using an activation function. Like linear regression, each neuron also has a bias which can be thought of as an input that always has the value 1.0 and it too must be weighted. Thus, the weights on the inputs are very much like the coefficients used in a regression equation. For example, a neuron may have two inputs in which case it requires three weights: one for each input and one for the bias. Weights are often initialized to small random values, such as values in the range 0 to 0.3 , although more complex initialization schemes can be used. Like linear regression, larger weights indicate increased complexity and fragility. It is desirable to keep weights in the network small and regularization techniques can be used. 
Figure 7.10: Neural network scheme showing the Input layer with $n$ inputs $I_{n}$, one hidden layer with $n$ neurons $H_{n}$ and one output layer with $n$ outputs $O_{n}$. [Source: the author.]

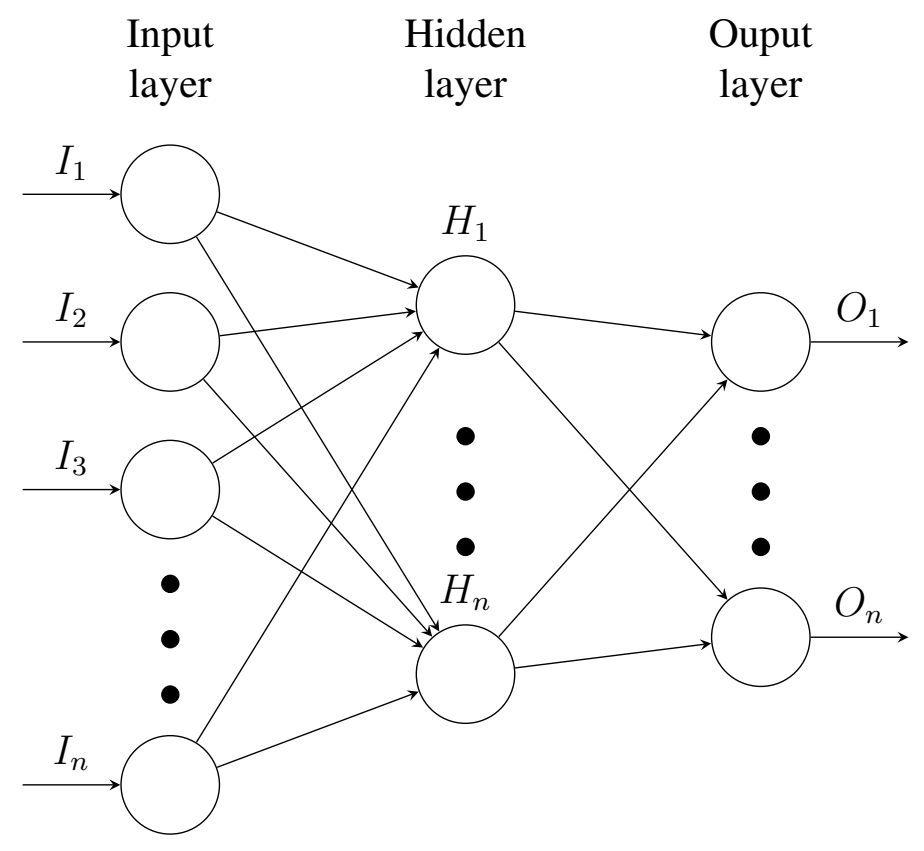

Formally, a one-hidden-layer MLP is a function $f: R^{D} \rightarrow R^{L}$, where $D$ is the size of input vector $x$ and $L$ is the size of the outut vector $f(x)$, such that

$$
f(x)=G\left[\boldsymbol{b}^{(2)}+\boldsymbol{W}^{(2)}\left(s\left(\boldsymbol{b}^{(1)}+\boldsymbol{W}^{(1)} x\right)\right)\right]
$$

with bias vectors $\boldsymbol{b}^{(1)}, \boldsymbol{b}^{(2)}$, weight matrices $\boldsymbol{W}^{(1)}, \boldsymbol{W}^{(2)}$ and activation functions $G$ and $s$. The vector $\boldsymbol{h}(x)=s\left(\boldsymbol{b}^{(1)}+\boldsymbol{W}^{(1)} x\right)$ constitutes the hidden layer. $\boldsymbol{W}^{(1)} \in R^{D \times D_{h}}$ is the weight matrix connecting the input vector to the hidden layer. Each column $\boldsymbol{W}_{\cdot i}^{(1)}$ represents the weights from the input units to the $i$-th hidden unit.

In this way, known rheological models were used in the generation of data for neural network training. The parameters used are shown in table 7.1. After obtaining the stress signal from these rheological models, a signal period obtained in UD-LAOS is decomposed into Chebyshev polynomials up to the seventh order. These coefficients are grouped as the input of the neural network. The target of the neural network for each of these inputs are the Chebyshev coefficients considering the LAOS test, with $\gamma_{D C}=0$. This process was conducted, as shown in figure 7.11, for Maxwell UPC, 
Table 7.1: Parameters used to train the neural network. The network was trainned using different frequencies in the range $0.1 \leq \omega \leq 1$. [Source: the author.]

\begin{tabular}{ccccccccc}
\hline & $\lambda^{\prime}$ & $\eta_{p}$ & $\alpha$ & $\lambda_{r}^{\prime}$ & $a$ & $\gamma_{D C}$ & $\gamma$ & $L_{f}$ \\
\hline Maxwell UPC & {$[0.5,10]$} & {$[0.002,2]$} & - & - & - & {$[0.1,1]$} & {$[0.1,1.5]$} & - \\
Giesekus & {$[0.5,10]$} & {$[0.002,2]$} & {$[0,0.5]$} & - & - & {$[0.1,1]$} & {$[0.1,1.5]$} & - \\
Oldroyd-B & {$[0.5,10]$} & {$[0.002,2]$} & - & {$[0.1,10]$} & - & {$[0.1,1]$} & {$[0.1,1.5]$} & - \\
FENE-P & {$[0.5,10]$} & {$[0.002,2]$} & - & - & {$[10,1000]$} & {$[0.1,1]$} & {$[0.1,1.5]$} & {$[10,1000]$}
\end{tabular}

Giesekus, Oldroyd-B and FENE-P models. 
Figure 7.11: Algorithm used for the data generation considering UPC Maxwell model. [Source: the author.]

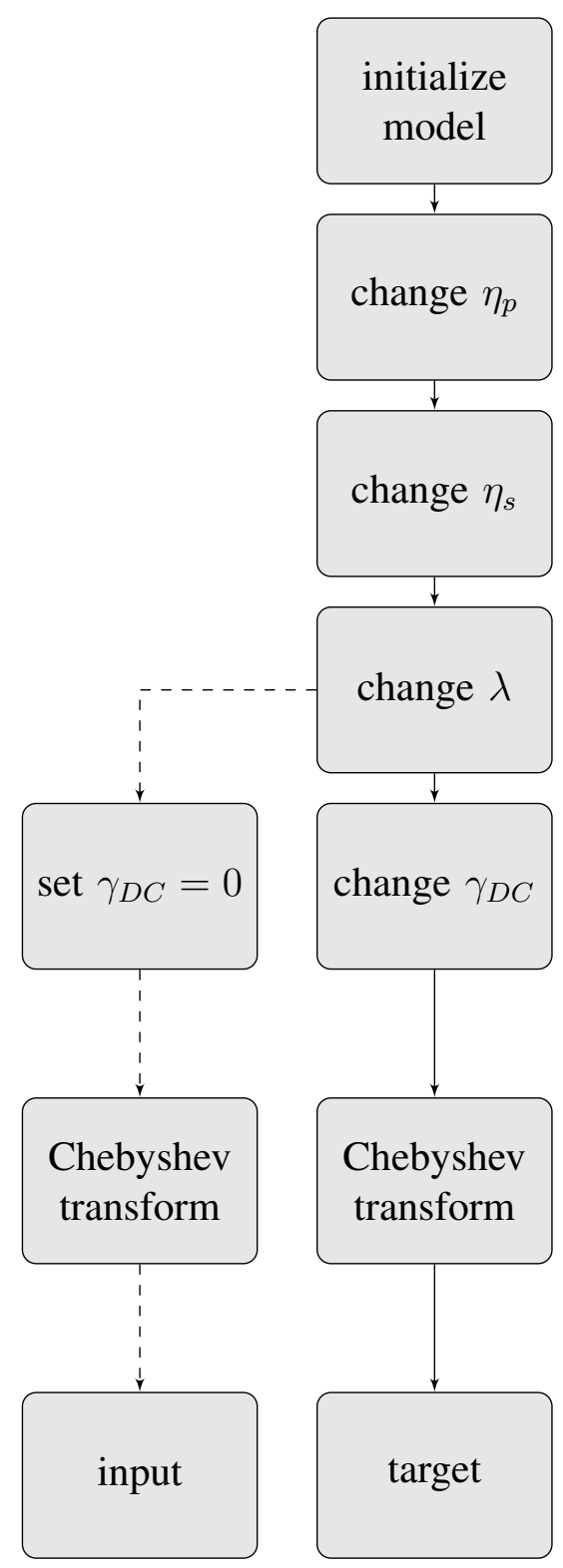




\subsubsection{Methodology validation}

The neural network was constructed with one input layer with input vector size $n=8$, containning the Chebyshev coefficients up to the 7-th order, based on rectifier linear unit function. The rectifier is an activation function defined as the positive part of its argument

$$
f\left(x_{i}\right)=x_{i}^{+}=\max \left(0, x_{i}\right)
$$

where $x_{i}$ is the input to a neuron. This is also known as a ramp function and is analogous to half-wave rectification in electrical engineering. The function is linear for values greater than zero, meaning it has a lot of the desirable properties of a linear activation function when training a neural network.

Since the data is not linearly separable, we use more than one hidden layer. Usually, two hidden layers can represent an arbitrary decision boundary to arbitrary accuracy with rational activation functions and can approximate any smooth mapping to any accuracy (HINTON et al.; HORNIK; CyBenko, 2006; 1991; 1989). On both hidden layers, the normalized exponential function (softmax) was used as activation. Softmax function outputs a vector that represents the probability distributions of a list of potential outcomes, namely

$$
f\left(x_{i}\right)=\frac{e^{x_{i}}}{\sum_{i} e^{x_{i}}} .
$$

The first hidden layer has 64 nodes and the second 32 nodes. Since the output layer also has 8 nodes, the network has 1,680 parameters to compute. The RMSprop algorithm (HINTON, 2014) was used to optimize the learning rate.

The objective of all optimizers is to reach the global minima where the cost function attains the least possible value. Let's consider, for instance, the gradient descent, a first-order iterative optimization algorithm, as example. To find a local minimum of a function using gradient descent, one takes steps proportional to the negative of the gradient (or approximate gradient) of the function at the current point. Basically, each time we find the gradient and update the values of weights and 
biases, we move closer to the optimum value. Before we start training the neural network, its cost would be high. Through each iteration of training the neural network, the cost reduces and moves closer to the global minimum value. In contrast, the cost function is not always convex and then there is a chance that the result is in a local minima and the loss might never converge to the global minimum value. In other words, the weights are updated as

$$
w_{\text {new }}=w_{\text {old }}-\alpha_{L R} \frac{\partial L}{\partial w_{\text {old }}}
$$

where $\alpha_{L R}$ is the learning rate and $\partial L / \partial w$ is the gradient of $L$, the loss function to minimise the component $w$. The rate used to change the weights and bias and reach the global minimum is called learning rate. Here, we can modify both learning rate and gradient component. Choosing a large value for the learning rate could lead to the impossibility to reach the global minima. However, small values of learning rate lead to high convergence times. The magnitude of the gradient can be very different for different weights and can change during learning. This makes it hard to choose a single global learning rate.

The RMSprop optimizer (HINTON et al., 2006) restricts the oscillations (or changes in weights and bias) in the vertical direction. Therefore, we can increase our learning rate and our algorithm could take larger steps in the horizontal direction converging faster. Moreover, it does not modify the gradient itself, but the learning rate. This algorithm update the weights as

$$
w_{\text {new }}=w_{\text {old }}-\frac{\alpha_{L R}}{\sqrt{S_{\text {new }}+\epsilon}} \frac{\partial L}{\partial w_{\text {old }}},
$$

where

$$
S_{\text {new }}=0.9 S_{\text {old }}+(1-0.9)\left(\frac{\partial L}{\partial w_{\text {old }}}\right)^{2}
$$

being $S_{\text {old }}$ the cumulative sum of current and past squared gradients and $\epsilon$ the "fuzz factor", a small floating point value to always ensure a non-zero division. 
It is important to emphasize that there is no mathematical theory for the definition of number of nodes, layers or optimization methods. Thus, after some tests, it was determined that these parameters are the most adequate considering the number of samples and the non-linearities of the problem.

The neural network was then trainned for 350 epochs using data from Maxwell UPC, Giesekus and Oldroyb-B (almost 200000 samples). The trainned network was tested for 216 samples of FENE-P model, resulting in a mean absolute error of 0.0193 . Two examples of the output are presented in figures 7.12 and 7.13. This low error shows that overfitting is not a problem in this configuration. Based on that, we are now able to use experimental data into the neural network.

Figure 7.12: Stress as a function of time for the output of the neural network. The solid line represents the expected target, while the dashed line represents the input of the network, namely the signal with $\gamma_{D C}$. The solid circles are the output of the neural network and represent the signal with DC after the nonlinear subtraction of $\gamma_{D C}$. The parameters are $\gamma_{D C}=0.3, \eta_{p}=1, \lambda^{\prime}=1, L=1$, $\gamma=1, a=1$ and $\omega=0.5$. [Source: the author.]

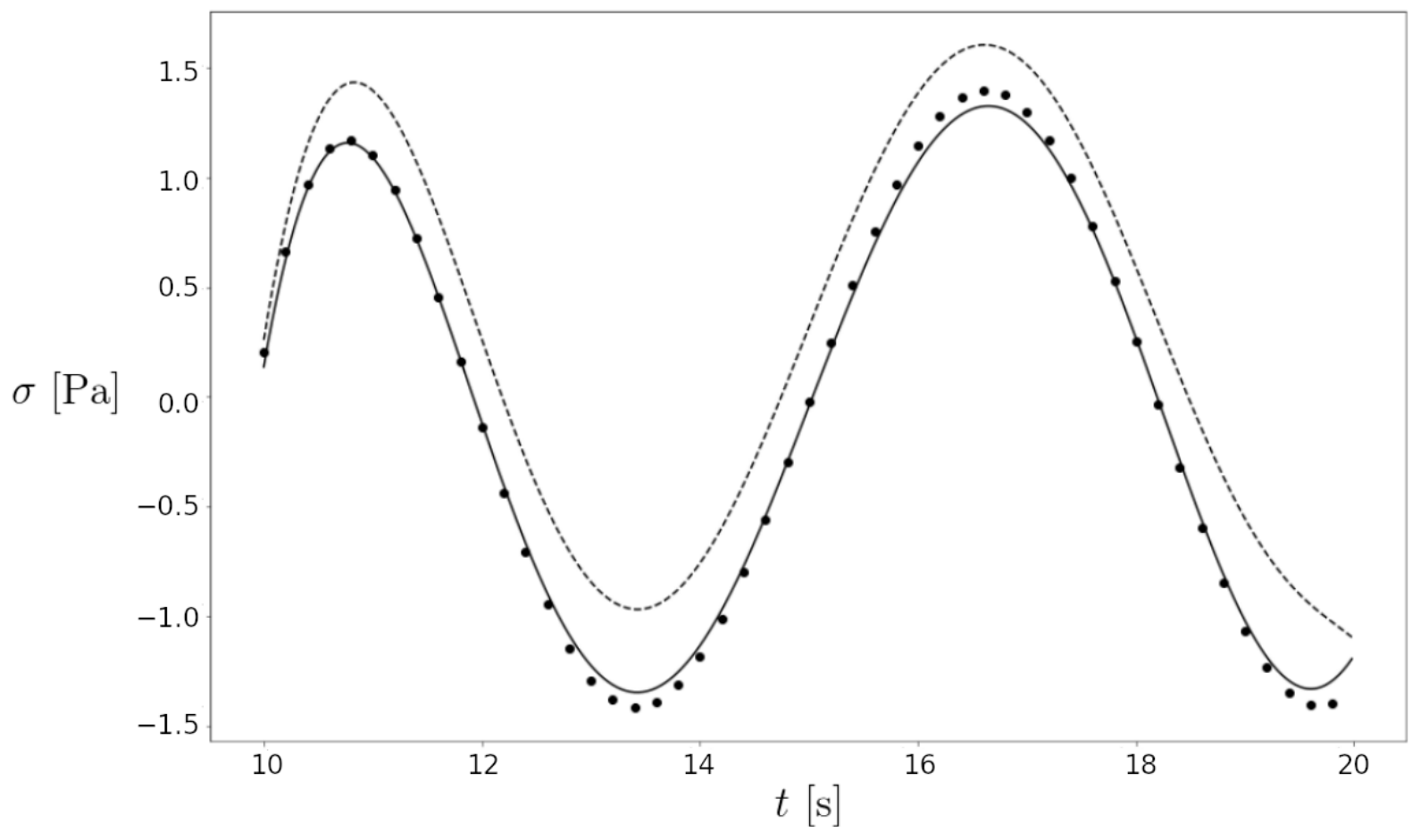


Figure 7.13: Stress as a function of time for the output of the neural network. The solid line represents the expected target, while the dashed line represents the input of the network, namely the signal with $\gamma_{D C}$. The solid circles are the output of the neural network and represent the signal with DC after the nonlinear subtraction of $\gamma_{D C}$. The parameters are $\gamma_{D C}=0.9, \eta_{p}=3, \lambda^{\prime}=7, L=1$, $\gamma=1, a=2$, and $\omega=0.5$. [Source: the author.]

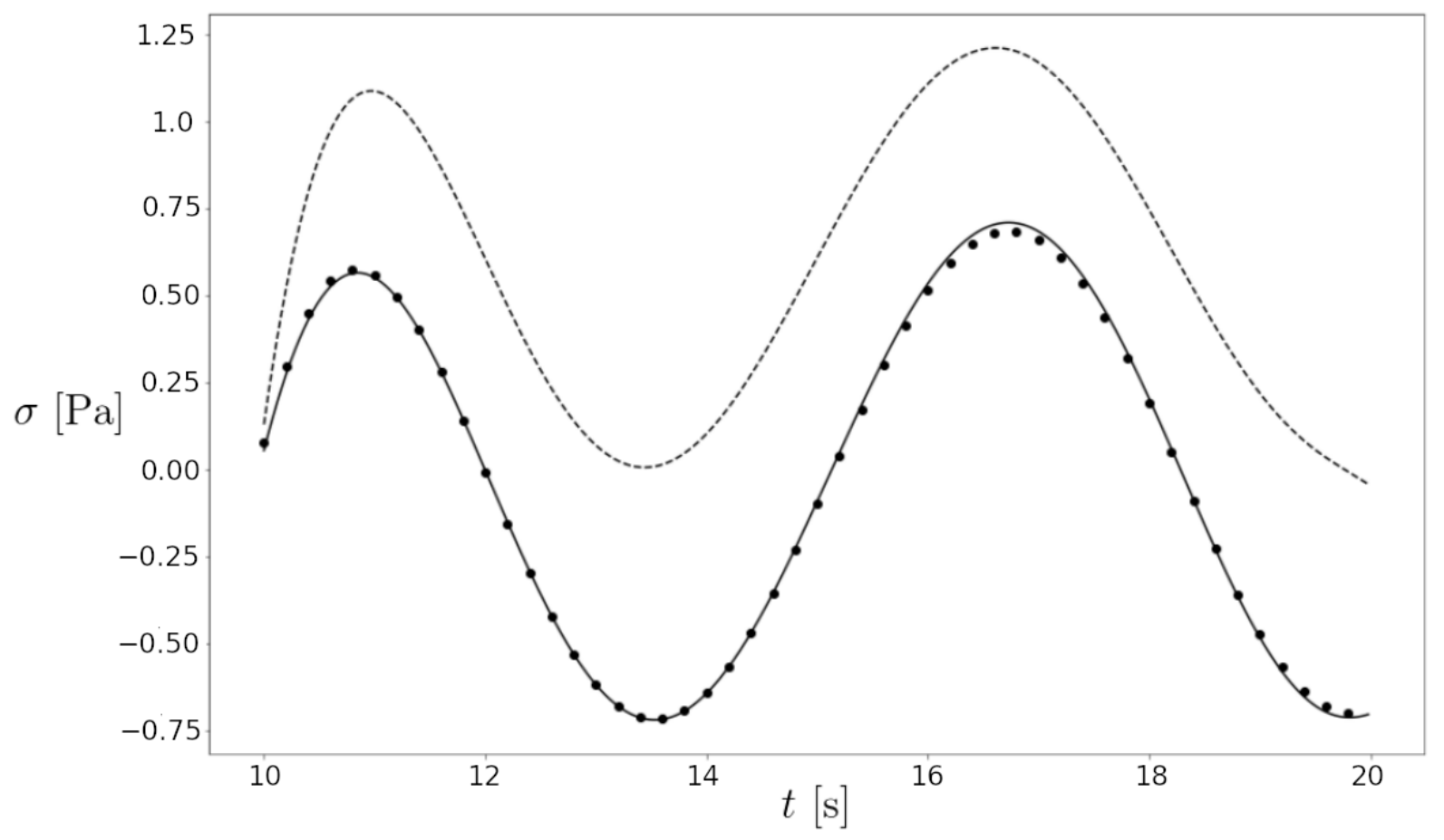

\subsubsection{Experimental data}

Since the neural network have shown good results, we are able to use the experimental data to remove the DC signal from the UD-LAOS results. The network was also trainned with FENE$\mathrm{P}$ model and tested with the Newtonian and polymeric suspension. Figure 7.14 shows the result obtained from the network for the Newtonian fluid. We observe that just subtracting the average from the velocity signal obtained from the particle tracking is not enough to reconstruct the LAOS experiment. There is a phase shift related to the UD-LAOS test. It is possible to see that, as expected, there is not phase shift between the output signal from the neural network (representing the strain-rate) and the pressure signal (representing the applied stress). However, for the polymeric suspension shown in figure 7.15, the agreement is remarkable and it is possible to see the expected phase shift related to the elastic behavior of the suspension. It means that we are able to obtain the rheological measurements such as compliances and fluidities. 
Figure 7.14: The plot shows the applied filtered pressure signal (dashed line), the normalized velocity subtracted from the average (solid line) and the output from the neural network, namely the expected velocity in a simulated LAOS experiment (solid circles) for a Newtonian fluid as a function of time, $t[\mathrm{~s}]$. [Source: the author.]

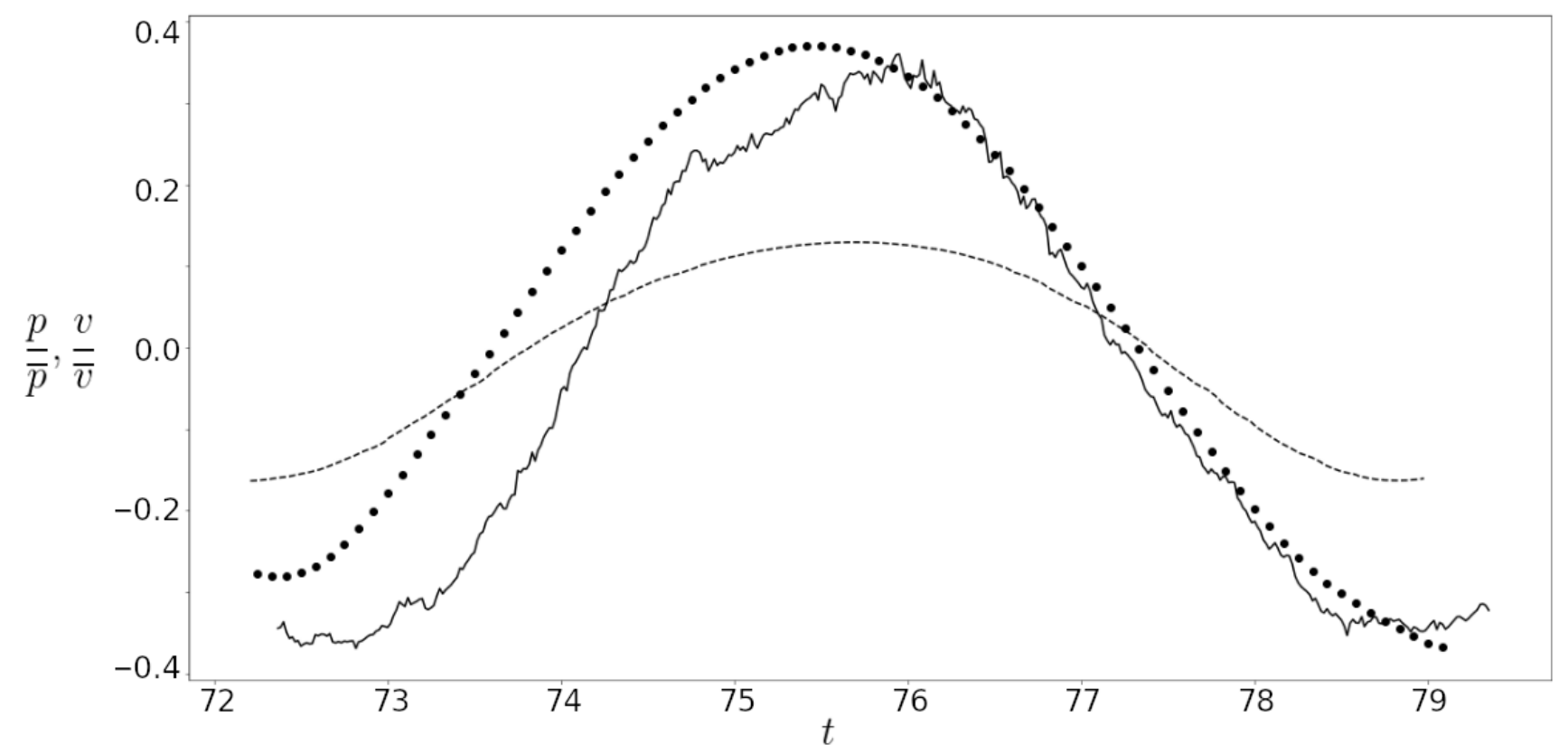

\subsection{Chapter conclusions}

Classical macroscopic rheometry techniques for measuring fluid properties in shear and elongation typically involve characteristic length-scales $\mathcal{O}(1 \mathrm{~mm})$, require sample volumes $\mathcal{O}(1 \mathrm{~mL})$ and probe deformation rates of perhaps up to $\mathcal{O}\left(100-1000 \mathrm{~s}^{-1}\right)$. While these methods are satisfactory for understanding the behaviour of many fluids in a wide variety of flows, there are circumstances when using devices with smaller length-scales to investigate rheological response may be advantageous. The use of unidirectional oscillatory flows in microrheology facilitates the study of suspensions of microorganisms.

This methodology presents a wide range of possibilities. Experiments in UD-LAOS are extremely important to understand how the orientation of the particles modifies the flow. In addition, this type of flow is the most common in in vivo situations in bioengineering. This DC bias in large 
Figure 7.15: The plot shows the applied filtered pressure signal (dashed line), the normalized velocity subtracted from the average (solid line) and the output from the neural network, namely the expected velocity in a simulated LAOS experiment (solid circles) for a non-Newtonian fluid as a function of time, $t[\mathrm{~s}]$. [Source: the author.]

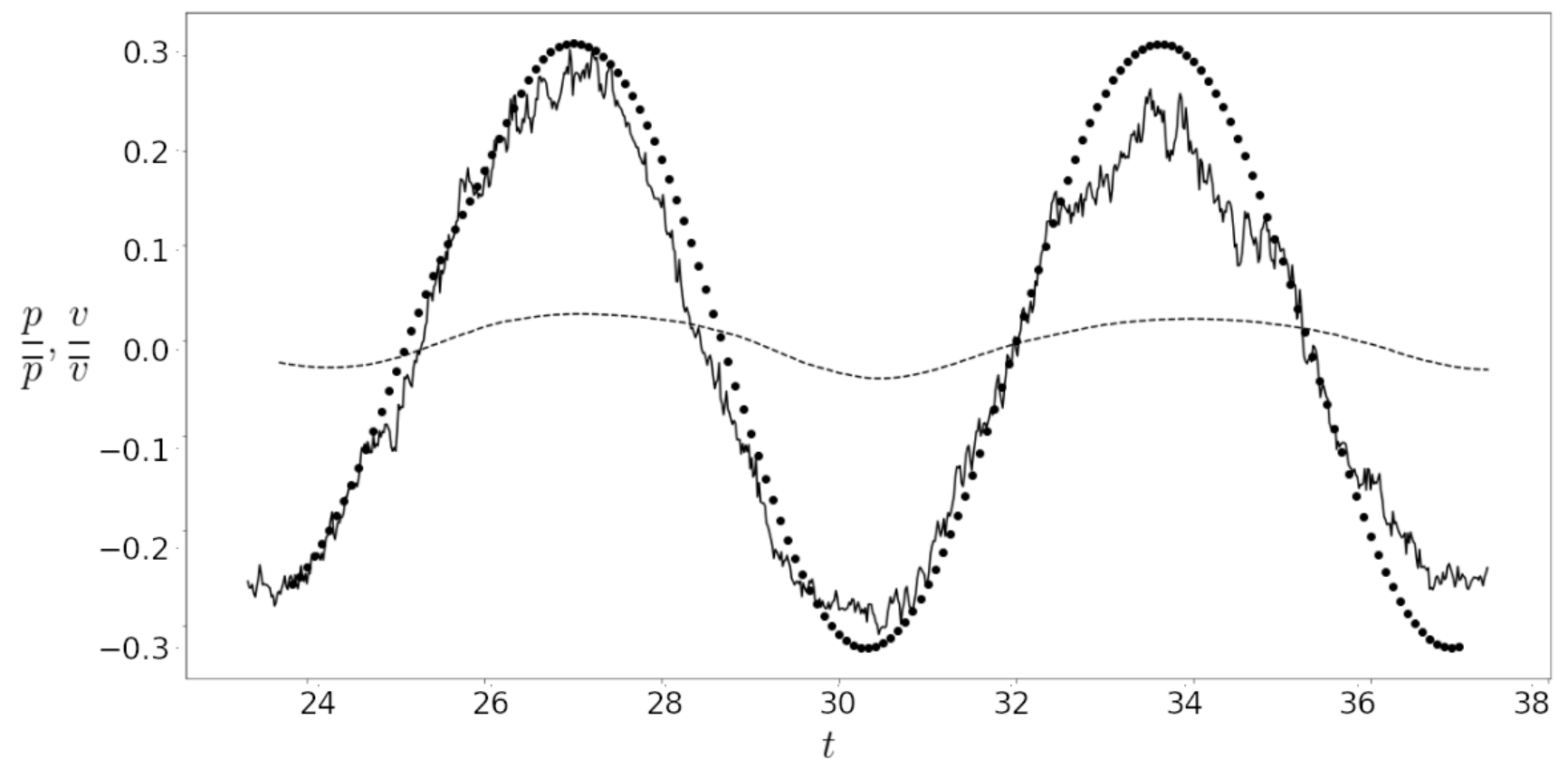

signals often causes a shift in the DC operating point that affects the amplitude, the amount of nonlinearity and the phase shift.

However, up to the present moment, there is no framework in the literature capable of analyzing quantitatively the rheological results obtained in UD-LAOS. Considering that in the nonlinear regime the DC component of the excitation signal propagates to all degrees of freedom in a non-separable fashion, the most appropriate way of dealing with the results is to remove the DC component. In this way, it is possible to analyze the results using the preferred methodology: stress decomposition, Chebyshev polynomials or Fourier transform. 


\section{Conclusion and future work}

Motivated by the growing interest in active suspensions, this thesis presented a new framework to fully understand the behavior of these living fluids. Several tools of signal analysis and artificial intelligence are used in a coupled way with experiments in macroreology and microreology, in order to describe the behavior of these suspensions. In addition, analytical tools are used for the proposition of a rheological model, based on the experimental data of kinematics and rheology. Simplification of the model is first tested through immersed boundary simulations. This protocol can also be applied to several types of passive and active suspensions. In this work, the motivation and the theoretical foundation are presented in chapters 1 and 2.

In the third chapter we have presented a statistical analysis on the kinematics-wave motion of a suspension of $C$. elegans in a gel-like medium. We have studied two different populations from a biological perspective of the surrounding medium. These populations consisted in a starving and a well-fed group of nematodes. We have found experimentally a linear correlation between the length and the wavelength of the individuals for both populations. We proposed a theoretical correlation to justify this linear dependence and the results have indicated that $C$. elegans indeed uses sinusoidal propulsion to move in creeping flow.

We have also found that, whereas the centroid of the individuals in both populations behaves nearly harmonically, their heads and tails evolve to highly non-harmonic motion. This nonlinear motion is used to break the time reversibility in which they are trapped due to their small sizes, known as kinematic reversibility in low Reynolds number flows. Another important finding of the present work is the discrepancy observed in the collective motion of both populations. We observed that well-fed individuals tend to move in the direction of E. coli collonies with less spreading in the surrounding medium. On the other hand, a starving population collectively behaves quite differently, seeking for food in several possible directions and with a much stronger head motion.

The study of the kinematic properties of the active particle is extremelly important to propose a constitutive model. Depending on the type of propulsion or orientation we may have different bulk results.

Considering that even the linear viscoelastic moduli of nematodes suspensions are poorly understood and time-dependent rheological studies of these types of suspensions have been scarce 
in the current literature, we presented a series of experimental rheological analysis of C. elegans suspensions in chapter 4 . We were able to observe both hydrodynamic and active mechanisms of the suspension during oscillatory shear and step strain tests.

Our experimental results have suggested a decrease in the fluids viscosity as the amount of nematodes increases until a certain critical volume fraction. We identified three important regions. The pure anisotropic region $(0 \leq \phi \leq 0.002)$ shows an approximately linear increase of the viscosity with nematode volume fraction. The viscosity is observed to follow the law of a rod body $\eta_{r} \sim K \phi(\ell / a)^{2}$, where $\ell / a$ is the anisotropic aspect ratio. In the active elastic region $(0.002 \leq \phi \leq 0.004)$ the active particles insert elastic energy by their intrinsic motion on the bulk suspension. The suspension viscosity decreased as a consequence of this elastic energy production by the particles in the case of pusher microorganisms. For $\phi \gtrsim 0.4 \%$, we have identified a region of structure formation, where collective viscous hydrodynamic particle-particle interactions that inhibit the elastic motion of the nematodes produce a nonlinear increasing of the suspension viscosity as the particle volume fraction increases.

At relatively high filling fractions microorganisms interact mostly through hydrodynamic entrainment induced by their swimming with respect to the ambient fluid. However, at low volume fractions, the fluctuations caused by the nematode's mobility, which is also generated by the elastic response that arise from the worm's body bending, tend to decrease the bulk viscosity. In the case of passive objects, a disturbance velocity only arises if an external force or flow field is imposed. This is unlike self-propelled particles, which drive fluid disturbances even in quiescent environments. The flow field a swimmer generates depends in a complicated manner on body kinematics, surface stresses, and possible surface slip.

The normal stress difference $\left(N_{1}\right)$ was also calculated. Unlike macromolecule suspensions, the active particle has a natural resistance to stretch, related to its intrinsic elasticity. Thus, there is anisotropy generated in the body of the nematode. However, as aggregates are formed, these structures tend to become anisotropic as they are stretching in the flow direction, causing an increase in the first difference of normal stress.

We have also observed an oscillatory behavior on the relaxation function. A similar behavior was previouslly observed in liquid crystals. As a matter of fact, the first normal-stress difference in shear flow of low-molecular-weight nematics is calculated from the Leslie-Ericksen theory. It is shown that, depending on the boundary orientation, the stress difference is either always negative, 
always positive or changes from negative to positive with increasing shear stress. This investigation was prompted by recent experimental studies demonstrating negative values of the first normalstress difference for polymeric liquid crystals. In this active suspension, not only the passive relaxation time must be observed, but also the active relaxation time. It clearly increases in higher volume fractions due to the collective behavior previously observed.

Even in the presence of an intrinsic elasticity in the nematode's membrane, considering the high aspect ratio of the nematode, we may simplify its shape by a slender filament. This simplification is important to propose a rheological constitutive model for the suspension. In this sense, in chapter 5 we analyzes how the kinematics of a flexible filament can be compared to that of a nematode. We used immersed boundary method to simulate the slender body. It was divided into a straight line and a third degree polynomial to promote bending. The main objective was to simulate the anguilliform locomotion of the nematode considering the positions of the interpolating mediary points, $p_{1}$ and $p_{2}$, of the polynomial part of the filament. As $p_{1}$ and $p_{2}$ were symmetrically and asymetrically changed, we computed trajectory, distance swam as a function of the number of strokes and velocity. We were then able to qualitativelly compare these results with the kinematic data presented in the third chapter.

When the mediary points were symmetrically changed, higher magnitudes of velocity and acceleration were observed when the distance from $p_{2}$ to $p_{1}$ was smaller, namely $\left(p_{1}, p_{2}\right)=(0.4,0.5)$. That means that the nematode curvature is smaller but it has more energy due to the size of the tail to use as propulsion. Moreover, the size of the center body, defined as $p_{2}-p_{1}$, is smaller and perceive less inertia, thus less energy is required to change the signal of the filament's curvature. That also means that the nematode tends to swim a longer distance with less strokes.

The mediary points were also changed asymetrically. These asymetries cause a difference in the trajectory. However, it is perceived that there is a large difference between these results and the experimental results observed. It is possible to notice that, despite the numerical capacities of this algorithm, for nematodes with no mutation, the simulations with symmetric changes in $p_{1}$ and $p_{2}$ are more compatible with experimental data. However, it is important to notice that the change of $p_{2}$ does not promote changes as the first case, when $p_{1}$ was also changed. Thus, the position of $p_{1}$ is probably more important than the size of the center body in terms of propulsion.

The stroke period can also be changed, regardless of the interpolation points (kept as $(p 1, p 2)=(0.1,0.9)$. The upstroke $t_{u}$ and the downstroke $t_{d}$ percentages of the total stroke pe- 
riod were asymetrically varied. As the upstroke percentage of a stroke decreases, it happens faster. However, although the swimmer that moves forward the fastest has the quickest upstroke, having a faster upstroke does not always lead to a faster forward swimming speed. Interestingly, due to the asymmetric UPS and DWS, the swimming velocity profiles were shown to be significantly different. In particular, the waveforms appear trimodal rather than bimodal, which were observed in the cases of varying the interpolation modes. For obvious reasons, the asymetric modification in period of UPS and DWS changes the trajectory of the filament.

The qualitative comparisons of the distance, trajectory and velocity curves of the immersed boundary simulations and the experimental data showed that it is possible to consider the nematode C. elegans as a flexible filament. In this sense, a new constitutive rheological model was developed.

Considering the orientational instabilities of the active suspension observed in the experiments, a nonlinear phenomenological model for neutrally buoyant force-free active suspension of nematodes was proposed and tested on the description of the linear and nonlinear viscoelastic behavior of this living suspension. Viscometric measurements of the simulated model were compared with the experiments in small amplitude oscillatory shear presented in chapter 4.

The model was tested with both only affine and also non-affine motion. That means that the local strain in a sample after deformation is not identical everywhere due to the concentration differences and the elastic disturbances caused by the active particle intrinsic motion. While the nematodes centers follow the macroscopic shear deformation affinely, the head and tail can deviate from the affine deformation field in order to minimize the global free energy. We observed that on LAOS tests and at concentrated suspensions, the non-affine motion must be considered and Gordon-Schowalter derivative used.

In this condition, $G^{\prime}$ and $G^{\prime \prime}$ obtained from the simulated model were compared with the experimental data and the constitutive equations were validated. Moreover, the stress response was very similar from those obtained through the rheometer. It is extremely important that the models are validated so that they can be tested in other flows. In general, it is far more practical to simulate a model and its bulk response than to perform experimental tests with live particles on different setups, which are in some cases extremelly complex.

Considering that the model was validated with experimental data, tests in the nonlinear regime were performed. Using the Lissajoud-Bowditch approach, the Pipkin diagrams were con- 
structed using different parameters. We observed that at sufficiently low Deborah number, the viscoelastic material has enough time to relax and behaves like a Newtonian liquid for small amplitudes. The material was driven into the nonlinear region, when $W i$ was increased to high values but De was kept constant.

The normal stress difference in LAOS was also studied. The normal stress increased when the nematodes formed a chain-like structure, until it is destroyed. Furthermore, using GordonSchowalter derivative, we were able to compute the second normal stress difference, $N_{2}$. Its negative sign is related to the nematode's elongation during shear at large amplitudes. Basically, the normal stresses arise due to anisotropy and orientation of particles and the proposed model is robust enough to compute both $N_{1}$ and $N_{2}$.

As mentioned before, classical macroscopic rheometry techniques for measuring fluid properties in shear and elongation typically involve characteristic length-scales $\mathcal{O}(1 \mathrm{~mm})$, require sample volumes $\mathcal{O}(1 \mathrm{~mL})$ and probe deformation rates of perhaps up to $\mathcal{O}\left(100-1000 \mathrm{~s}^{-1}\right)$. While these methods are satisfactory for understanding the behaviour of many fluids in a wide variety of flows, there are circumstances when using devices with smaller length-scales to investigate rheological response may be advantageous. Furthermore, usual macroscopic rheometry does not allow us to obtain velocity profile and track the particles. Based on that, a framework for analysis of rheological properties of active suspensions using microscopic rheometry was proposed on chapter 7 .

The protocol developed to use the microfluidic rheometer constructed at University of Pennsylvania presents unique results in the literature involving neural networks. This rheometer, based on pulsatile flow, which is constantly present in vivo, allows studying the reaction of the particles in non-reversible flows. However, the UD-LAOS flow adds non-linearities to the response of the fluid due to the DC component of strain (or stress).

Firstly, we analyzed mathematically whether the same decomposition based on Chebyshev polynomials could be made. We identified that nonlinearities spread throughout the harmonic spectrum. In this sense, seeking to remove the DC signal and emulate the behavior of a suspension under LAOS test from the results obtained in UD-LAOS, a neural network was trained with several viscoelastic rheological models well established in the literature.

A polymeric viscoelastic suspension was tested in that network and it was observed that it correctly removed the non-linearities of the response concerning the addition of the DC signal and 
the non-reversibility of this type of flow. From this, it was possible to correctly identify the phase shift between the excitation and response signals, which allows the viscometric quantities to be obtained such as compliances and fluidities, viscosities and elastic moduli.

In addition to the viscometric measurements, the particles were also tracked based on the IDL programming language and the velocity and shear rate profile were measured for both Newtonian and non-Newtonian suspensions.

In general, this thesis presents a complete picture of how to analyze a suspension of active (or passive) particles and can be extended to any type of microorganism or molecule. First, it is necessary to understand how the particle behaves kinetically, so as to understand each organism as one. With this type of analysis it is possible to determine the type of active particle and how its propulsion occurs, in addition to the type of instabilities it causes in the surrounding fluid.

After identifying these kinematic characteristics, it is necessary to understand how the behavior of several particles modifies the fluid and its rheological measurements. In this sense, experiments in macroreology are important to understand the continuum. However, active particles are usually complex organisms in physical structure, having intrinsic viscoelastic characteristics and unconventional shapes, besides having their own propulsion mechanisms. For this reason, in some cases some simplifications are necessary in order to consider only the crucial aspects of these particles.

In order to determine if these simplifications are comparable to the reality of the active particles, simulations may be performed and compared with experimental data. As we observed, the nematodes can be simplified as a flexible filament based on the immersed boundary simulations.

The rheological model is then proposed considering the simplifications performed. As mentioned, active particles are difficult to use in experiments due to its sensibility and the complex setup necessary to perform tests considering different flows. It is easier to study the behavior of these living fluids considering constitutive models, which must be proposed based on the simplifications. However, it is very important to validate the model using experimental data in linear regime before performing nonlinear experiments.

To conclude the framework, microrheology can be used in order to obtain the velocity and shear rate profiles, track the particles and observe their response to shear flows commonly present 
in vivo, such as pulsatile flows.

This complete framework proposed is completely new in the literature and allows the entire spectrum of the suspension to be studied from the particle as an independent agent, to its collective behavior in the various types of experimental flows and setups following other well stablished frameworks of rheological analysis such as Fourier transform, stress decomposition and Chebyshev polynomials.

In the future, different other types of suspensions will be studied: one example is the use of methylotrophic bacteria that produce biopolymers. Brazil is responsible for the production of 6.5 million tons of plastics in the world, mainly in the civil construction, food and automotive sectors. Even more worrying is that today, about $60 \%$ of what is produced are non-biodegradable plastics. An environmentally correct alternative to the production of petroderivatives is bioplastics, or also called biopolymers. In particular, the polyhydroxyalkanoates (PHAs) represent a class of polymers which may serve as potential substitutes for conventional plastics. This is because PHAs are produced naturally by bacteria through renewable sources, are biodegradable and also biocompatible, that is, they do not produce toxic byproducts during their degradation process. These PHAs are a class of biopolymers naturally produced by bacteria as energy reserve granules.

This class of bacteria is extremely interesting to be studied using this framework. It is necessary to understand how they behave kinetically, since there are several microorganisms present together. In addition, the presence of the bacteria before and after the production of PHAs changes the rheology of the suspension fluid. Besides that, because they are complex microorganisms and act together, the models need to be simplified and then simulated to determine the validity of these simplifications. After this step it is possible to propose a constitutive model and study the suspensions in all types of flow and conditions. This greatly facilitates the experimental process that is nowadays done with samples taken from nature (e.g. samples from Santos bay (CARDoso, 2017)). Finally, this type of suspension can be studied in the microfluidic rheometer and it is possible to analyze the trajectory of the particles during flow, identify the velocity profile and also calculate the viscometric characteristics of the suspension while the orientation of the bacteria is analyzed. By analyzing all these aspects of this suspension, for example, it is possible to understand what are the best conditions of concentration, temperature, interaction between microorganisms, type of flow or excitation applied to increase the production of the biopolymers, producing a sustainable alternative to common polymers. 
In addition, there are other processes within the proposed framework that can be improved. For example, during rheological analysis, it is possible to perform other tests such as the creep test instead of the step strain test. Some exprimental errors related to low torques and inertial problems can be overcome in this way.

Another limitation that may be tackled is that the trained neural network identifies only viscoelastic fluids. Thixotropic suspensions, for example, will not be correctly computed by this network. Currently, different neural networks need to be trained for different types of fluids, considering existing rheological models. In this sense, it is considered that the training of a more robust neural network can overcome this problem of previous identification of the type of fluid. In addition, other types of orthogonal decomposition, other than Chebyshev polynomials or Fourier series, can be used to train new machine learning algorithms.

In general, a few details of the proposed framework can be improved punctually. However, each adaptation must be made based on the characteristics of the active particle being studied. All in all, this work presents several new tools that were still lacking in the literature for understanding active suspensions. A whole new branch was developed, opening several opportunities for future work. 


\section{References}

ABKENAR, M. and MARX, K. Collective behavior of penetrable self-propelled rods in two dimensions. Physical Review E, v. 88, n. 6, 062314, 2013.

ACIERNO, D.; LA MANTIA, F. and MARRUCCI, G. A non-linear viscoelastic model with structure-dependent relaxation times: III. comparison with l.d. polyethylene creep and recoil data. Journal of Non-Newtonian Fluid Mechanics, v. 209, n. 3, 271-280, 1977.

AGASSANT, J.F. and PIAU, J.M. Rheology for Polymer Melt Processing. Elsevier, 1996.

AKEMA, M.J.; HUNTER-ENSOR, M.; RINGSTAD, N. and HORVITZ, H.R. Tyramine Functions Independently of Octopamine in the Caenorhabditis elegans Nervous System. Neuron, v. 46, $247-$ 260, 2005.

AL-HADITHI, T.; BARNES, H. and WALTERS, K. The relationship between the linear (oscillatory) and nonlinear (steady-state) flow properties of a series of polymer and colloidal systems. Colloid and Polymer Science, v. 270, 40-46, 1992a.

AL-HADITHI, T.S.R.; BARNES, H.A. and WALTERS, K. The relationship between the linear (oscillatory) and nonlinear (steady-state) flow properties of a series of polymer and colloidal systems. Colloid and Polymer Science, v. 270, 40-46, 1992b.

AL-HADITHIH, T.S.R.; BARNESK, A. and WALTERS, K. The relationship between the linear (oscillatory) and nonlinear (steady-state) flow properties of a series of polymer and colloidal systems. Colloid and Polymer Science, v. 270, 40-46, 1992.

ARMSTRONG, M.J.; BERIS, A.N.; ROGERS, S.A. and WAGNER, N.J. Dynamic shear rheology 
of a thixotropic suspension: Comparison of an improved structure-based model with large amplitude oscillatory shear experiments. Journal of Rheology, v. 60, 433-450, 2016.

BACKHOLM, M.; RYU, W.S. and DALNOKI-VERESS, K. Viscoelastic properties of the nematode Caenorhabditis elegans, a self-similar, shear-thinning worm. Proceedings of the National Academy of Sciences of the USA, v. 110, n. 12, 4528-4533, 2013.

BALASUBRAMANIAN, S.; KAGAN, D.; HU, C.M.; CAMPUZANO, S.; LOBO-CASTANON, M.J.; LIM, N.; KANG, D.; ZIMMERMAN, M.; ZHANG, L. e WANG, J. Micromachine-enabled capture and isolation of cancer cells in complex media. Angewandte Chemie International Edition, v. 50, 4161-4164, 2011.

BASKARAN, A. and MARCHETTI, M. Statistical mechanics and hydrodynamics of bacterial suspensions. Proceedings of the National Academy of Sciences of the USA, v. 106, n. 37, 15567$15572,2009$.

BATCHELOR, G. The effect of Brownian motion on the bulk stress in a suspension of spherical particles. Journal of Fluid Mechanics, v. 83, n. 1, 97-117, 1977.

BATTISTA, N.A.; BAIRD, A.J. and MILLER, L.A. A mathematical model and MATLAB code for muscle-fluid-structure simulations. Integrative and Comparative Biology, v. 55, 901-11, 2015.

BATTISTA, N.A.; STRICKLAND, W.C.; BARRETT, A. and MILLER, L.A. IB2d Reloaded: a more powerful Python and MATLAB implementation of the immersed boundary method. Mathematical Methods in the Applied Sciences, v. 41, 8455-8480, 2018.

BATTISTA, N.A.; STRICKLAND, W.C. and MILLER, L.A. IB2d:a Python and MATLAB implementation of the immersed boundary method. Bioinspiration and Biomemetics, v. 12, n. 3, 036003, 2017.

BAUGH, L.R. To grow or not to grow: Nutritional control of development during Caenorhabditis elegans L1 arrest. Genetics, v. 194, 539-555, 2013. 
BECHTEL, T. and KHAIR, A. Linear viscoelasticity of a dilute active suspension. Rheological Acta, v. 56, n. 2, 149-160, 2017.

BERNE, B.J. and PECORA, R. Dynamic Light Scattering. Wiley, 1976.

BERRI, S.; BOYLE, J.; TASSIERI, M.; HOPE, I. and COHEN, N. Forward locomotion of the nematode C. elegans is achieved through modulation of a single gait. HFSP Journal, v. 3, n. 3, 186-193, 2009.

BERRI, S. and ET AL. Forward locomotion of the nematode C. elegans is achieved through modulation of a single gait. HFSP Journal, v. 3, n. 3, 2010.

BIRD, R.B.; DOTSON, P.J. and JOHNSON, N.L. Polymer solution rheology based on a finitely extensible bead-spring chain model. Journal Non-Newtonian Fluid Mechanics, v. 7, 213-235, 1980.

BISWAS, S.; MURATA, T.; EBINA, Y.; OKADA, H. and MIKI, T. A method for motion compensation of a moving nematode Caenorhabditis elegans and its application to frequency analysis of pharyngeal pulsation. Journal of Biotechnology, v. 61, n. 3, 175-189, 1998.

BLAKE, J. Hydrodynamic calculations on the movements of cilia and flagella. II. Opalina. Journal of theoretical biology, v. 52, n. 1, 67-82, 1975.

BLAKE, J. and SLEIGH, M. Mechanics of Ciliary Locomotion. J. Theor. Biol., v. 49, n. 1, 183-203, 1974.

BOYLE, J.H.; JOHN, S. and DEHGHANI-SANIJ, A.A. Adaptive Undulatory Locomotion of a C. elegans Inspired Robot. IEEE/ASME Transactions on Mechatronics, v. 18, n. 6, 439-448, 2013.

BRATANOV, V.; JENKO, F. and FREY, E. New class of turbulence in active fluids. PNAS, v. 8, n. 112(49), 15048-53, 2015. 
BRENNEN, C. and WINET, H. Fluid Mechanics of Propulsion by Cilia and Flagella. Annual Review of Fluid Mechanics, v. 9, 339-398, 1977.

BROWN, W. Dynamic Light Scattering: The Methods and Some Applications. 1993.

CALHOUN, A.; CHALASANI, S. and SHARPEE, T. Maximally informative foraging by Caenorhabditis elegans. eLife, v. 3, e04220, 2014.

CALVO-MARZAL，P; SATTAYASAMITSATHIT, S.; BALASUBRAMANIAN, S.; WINDMILLER, J.; DAO, C. and WANG, J. Propulsion of nanowire diodes. Chemical Communications, v. 46, n. 10, 1623-1624, 2010.

CARDOSO, L.O.B. Produção de Polihidroxibutirato (PHB) por Bactérias Metilotróficas. Master dissertation, 2017.

CASSADA, R.C. and RUSSEL, R.L. The dauer larva, a post-embryonic developmental variant of the nematode Caenorhabditis elegans. Developmental Biology, v. 46, 326-342, 1975.

CATES, M.; FIELDING, S.; MARENDUZZO, D.; ORLANDINI, E. and YEOMANS, J.M. Shearing Active Gels Close to the Isotropic-Nematic Transition. Physical Review Letters, v. 101, n. 6, 068102, 2008.

CHANG, S.T.; PAUNOV, V.N.; PETSEV, D.N. e VELEV, O.D. Remotely powered self-propelling particles and micropumps based on miniature diodes. Nature materials, v. 6, 235-240, 2007.

CHEN, B.L.; HALL, D.H. and CHKLOVSKII, D.B. Wiring optimization can relate neuronal structure and function. Proceedings of the National Academy of Sciences, v. 103, 4723-4728, 2006.

CHILCOTT, M.D. and RALLISON, J.M. Creeping flow of dilute polymer solutions past cylinders and spheres. Journal Non-Newtonian Fluid Mechanics, v. 29, 381-432, 1988. 
CHILDRESS, S. Mechanics of swimming and flying. Cambridge University Press, 1981.

CHO, K.S.; HYUN, K.; AHN, K.H. e LEE, S. A geometrical interpretation of large amplitude oscillatory shear response. Journal of Rheology, v. 49, n. 3, 747, 2005.

CICUTA, P. and DONALD, A.M. Microrheology: a review of the method andapplications. Soft Matter, v. 3, 1449-1455, 2007.

COOLEY, J.W. and TUKEY, J.W. An algorithm for the machine calculation of complex fourier series. Mathematics of Computation, v. 19, 297-301, 1965.

COX, W.P. and MERZ, E.H. Correlation of dynamic and steady flow properties. Journal of Polymeric Science, v. 28, 619, 1958.

CROCKER, J. and GRIER, D.G. Methods of Digital Video Microscopy for Colloidal Studies. Journal of Colloid and Interface Science, v. 179, 298, 1996.

CYBENKO, G. Approximations by superpositions of sigmoidal functions. Mathematics of Control, Signals, and Systems, v. 2, n. 4, 303-314, 1989.

DAUPTAIN, A.; FAVIER, J. and BOTTARO, A. Hydrodynamics of ciliary propulsion. Journal of Fluids and Structures, v. 24, n. 8, 1156-1165, 2008.

DEVILlE, M. and GATSKY, T.B. Mathematical Modeling for Complex Fluids and Flows. Springer Berlin Heidelberg, 2012.

DOMBROWSKI, C.; CISNEROS, L.; CHATKAEW, S.; GOLDSTEIN, R.E. and KESSLER, J.O. Self-concentration and large-scale coherence in bacterial dynamics. Physical Review Letters, v. 93, n. 9, 0981032, 2004.

DOOSTMOHAMMADI, A.; IGNES-MULLOL, J.; YEOMANS, J.M. and SAGUES, F. Active 
Nematics. Nature Communications, v. 9, 3246, 2018.

DROZDOV, A.D. and GOTTLIEB, M. Constitutive equations for non-affine polymer networks with slippage of chains. Continuum Mechanics and Thermodynamics, v. 17, n. 3, 217-246, 2005.

DUMITRIU, S. Polysaccharides: Structural Diversity and Functional Versatility. Marcel Dekker, 1998.

DUNKEL, J.; HEIDENREICH, S.; DRESCHER, K.; WENSINK, H.H.; BAR, M. and GOLDSTEIN, R.E. Fluid Dynamics of Bacterial Turbulence. Physical Review Letters, v. 110, n. 22, 228102, 2013.

EBBENS, S. and HOWSE, J. In the pursuit of propulsion at the nanoscale. Soft Matter, v. 6, 726-738, 2010.

EINSTEIN, A. Eine neue Bestimmung der Molekuledimensionen. Annalen der physik, v. 19, 289-306, 1905.

ELGETTI, J.; WINKLER, R.G. and GOMPER, G. Physics of microswimmers single particle motion and collective behavior: a review. Reports on Progress in Physics, v. 78, 056601, 2015.

ERBAN, R. and OTHMER, H. From Individual to Collective Behavior in Bacterial Chemotaxis Read More: https://epubs.siam.org/doi/abs/10.1137/S0036139903433232. Journal of Applied Mathematics, v. 65, n. 5, 361-391, 2006.

EWOLDT, R.H.; HOSOI, A.E. and MCKINLEY, G.H. An Ontology for Large Amplitude Oscillatory Shear Flow. AIP Conference Proceedings, v. 1027, n. 1, 2008a.

EWOLDT, R.H.; HOSOI, A.E. and MCKINLEY, G.H. New measures for characterizing nonlinear viscoelasticity in large amplitude oscillatory shear. Journal of Rheology, v. 52, n. 6, 1427-1458, 2008b. 
EWOLDT, R.H. and MCKINLEY, G.H. On secondary loops in LAOS via self-intersection of Lissajous-Bowditch curves. Rheologica Acta, v. 49, 213-219, 2010.

EWOLDT, R.H.; WINTER, P. and MCKINLEY, G.H. MITlaos - MATLAB based data analysis software for characterizing nonlinear viscoelastic responses to oscillatory shear strain. v2.1 Beta, 2007.

FANG-YEN, C.; WYART, M.; XIE, J.; KAWAI, R.; KODGER, T.; CHEN, S.; WEN, Q. and SAMUEL, A.D.T. Biomechanical analysis of gait adaptation in the nematode Caenorhabditis elegans. PNAS, v. 107, n. 47, 2010.

FAUCI, L. and DILLON, R. A Review of Material Frame-Indifference in Mechanics. Applied Mechanics Review, v. 51, n. 8, 489-504, 1998.

FAUCI, L. and DILLON, R. Biofluidmechanics of reproduction. Annual Review of Fluid Mechanics, v. 38, 371-394, 2006.

FIELDING, S.; MARENDUZZO, D. and CATES, M. Nonlinear dynamics and rheology of active fluids: simulations in two dimensions. Physical Review E, v. 83, 041910, 2011.

FODOR, E. and ET AL. How Far from Equilibrium Is Active Matter? Physics Review Letters, v. 117, n. 3, 038103-1, 2016.

FORSTER, D. Microscopic Theory of Flow Alignment in Nematic Liquid Crystals. Physical Review Letters, v. 32, n. 21, 1161-1164, 1974.

FRANCIS, C.L.; RYAN, T.A.; JONES, B.D.; SMITH, S. and FALKOW, S. Active Nematics. Nature, v. 364, 639-642, 1993.

FU, H.; WOLGEMUTH, C. and POWERS, T. Physics of Fluids, v. 21, 033102, 2009. 
GACHELIN, J. and ET AL. Collective motion in an active suspension of Escherichia coli bacteria. New Journal of Physics, v. 16, n. 2, 025003, 2014.

GAO, W. and WANG, J. Synthetic micro/nanomotors in drug delivery. Nanoscale, v. 18, 1591815919, 2014.

GARCIA-GRADILLA, V.; OROZCO, J.; SATTAYASAMITSATHIT, S.; SOTO, F.; KURALAY, F.; POURAZARY, A.; KATZENBERG, A.; GAO, W.; SHEN, Y. and WANG, J. Functionalized ultrasound-propelled magnetically guided nanomotors: toward practical biomedical applications. ACS Nano, v. 7, n. 10, 9232-9240, 2013.

GIBBS, J. and ZHAO, Y. Autonomously motile catalytic nanomotors by bubble propulsion. Applied Physics Letters, v. 94, 163104, 2009.

GIESEKUS, H. A Simple Constitutive Equation for Polymer Fluids Based on the Concept of Deformation-Dependent Tensorial Mobility. Journal of Non-Newtonian Fluid Mechanics, v. 11, 69-109, 1982.

GIESEKUS, H. Constitutive Equations for Polymer Fluids Based on the Concept of ConfigurationDependent Molecular Mobility: A Generalized Mean-Configuration Model. Journal of NonNewtonian Fluid Mechanics, v. 17, 349-372, 1985.

GIOMI, L.; MARCHETTI, M. and LIVERPOOL, T. Complex Spontaneous Flows and Concentration Banding in Active Polar Films. Physical Review Letters, v. 101, 198101, 2008.

GOSH, R. and EMMONS, S.W. Episodic swimming behavior in the nematode C. elegans. Journal of Experimental Biology, v. 211, 3703-3711, 2008.

GRAY, J. and LISSMANN, H. The Locomotion of Nematodes. Journal of Experimental Biology, v. 23, 135-154, 1964.

GRULLER, H.; DEWALD, U. and EBERHARDT, M. Nematic liquid crystals formed by living 
amoeboid cells. 11, v. 100, 187-192, 2012.

GUPTA, R.K. Polymer and Composite Rheology. CRC Press, 2010.

GYRYA, V.; ARANSON, I.; BERLYAND, L. e KARPEEV, D. A model of hydrodynamic interaction between swimming bacteria. Bulletin of Mathematical Biology, v. 72, n. 1, 148-183, 2009.

HATWALNE, Y.; RAMASWAMY, S.; RAO, M. e SIMHA, R. Rheology of Active-Particle Suspensions. Physical Review Letters, v. 92, 118101, 2004.

HERNANDEZ-ORTIZ, J.; STOLTZ, C. and GRAHAM, M. Transport and Collective Dynamics in Suspensions of Confined Swimming Particles. Physical Review Letters, v. 95, n. 20, 204501, 2005.

HEUSSINGER, C. and FREY, E. Stiff Polymers, Foams, and Fiber Networks. PhysicaL Review Letters, v. 96, 017802, 2006.

HINSCH, H. and FREY, E. Non-Affine Shear Modulus in Entangled Networks of Semiflexible Polymers. Soft Condensed Matter, v. arXiv:0907.1875, 2009.

HINTON, G.E. Neural networks for machine learning - lecture 6a, overview of mini-batch gradient descent. 2014.

URL: https://www.cs.toronto.edu/tijmen/csc321/slides/

HINTON, G.E.; OSINDERO, S. and TEH, Y.W. A Fast Learning Algorithm for Deep Belief Nets. Neural Computation, v. 18, n. 7, 1527-1554, 2006.

HOHENEGGER, C. and SHELley, M. Stability of active suspensions. Physical Review E, v. 81, n. 4, 046311, 2010. 
HORNIK, K. Approximation Capabilities of Multilayer Feedforward Networks. Neural Networks, v. 4, n. 2, 251-257, 1991.

HORSTMANN, J.A. The Role of Flagella and Bacterial Motility in Virulence of Salmonella. PhD Thesis, p. Technische Universitat Braunschweig, 2017.

HYUN, K.; AHN, K.H.; LEE, S.J.; SUGIMOTO, M. and KOYAMA, K. Degree of branching of polypropylene measuredfrom Fourier-transform rheology. Journal of Rheology, v. 46, 123-129, 2006.

HYUN, K.; BAIK, E.S.; AHN, K.H.; LEE, S.J.; SUGIMOTO, M. and KOYAMA, K. Fouriertransform rheology under medium amplitude oscillatory shear for linear and branched polymer melts. Journal of Rheology, v. 51, n. 6, 1319-1342, 2007.

HYUN, K. and WILHELM, M. Establishing a new mechanical nonlinear coefficient Q from FT-Rheology: First investigation of entangled linear and comb polymer model system. Macromolecules, v. 42, n. 1, 411-422, 2009.

INGHAM, C.J. and BEN JACOB, E. Swarming and complex pattern formation in Paenibacillus vortex studied by imaging and tracking cells. BMC Microbiology, v. 8, e00103-13, 2008.

ISAACSON, E. and KELLER, H.B. Analysis of numerical methods. Dover Publications, 1994.

ISHIKAWA, T. Suspension biomechanicsof swimming microbes. Journal of the Royal Society Interface, v. 6, 815-834, 2009.

ISHIKAWA, T. and PEDLEY, T. Dispersion of model microorganisms swimming in a nonuniform suspension. Physical Review Letters, v. 90, n. 3, 033008, 2014.

JEYASEELAN, R.S. and GIACOMIN, A.J. Network theory for polymer solutions in large amplitude oscillatory shear. Journal of Non-Newtonian Fluid Mechanics, v. 148, n. 1-3, 24-32, 2008. 
JUAREZ, G.; LU, K.; SZNITMAN, J. e ARRATIA, P.E. Motility of small nematodes in wet granular media. Europhys. Lett., v. 92, 44002, 2010.

KAGAN, D.; BENCHIMOL, M.J.; CLAUSSEN, J.C.; CHULUUN-ERDENE, E.; ESENER, S. and WANG, J. Acoustic droplet vaporization and propulsion of perfluorocarbon-loaded microbullets for targeted tissue penetration and deformation. Angewandte Chemie International Edition, v. 51, 7519-7522, 2012.

KAGAN, D.; CAMPUZANO, S.; BALASUBRAMANIAN, S.; KURALAY, F.; FLECHSIG, G.U. and WANG, J. Functionalized micromachines for selective and rapid isolation of nucleic acid targets from complex samples. Nano Letters, v. 11, 2083-2087, 2011.

KEIM, N.C.; GARCIA, M. and ARRATIA, P.E. Fluid Elasticity Can Enable Propulsion at Low Reynolds Number. Physics Fluids, v. 24, n. 8, 081703, 2012.

KELLER, S. and SEGEL, L. Model for chemotaxis. Journal of Theoretical Biology, v. 30, n. 2, 225-234, 1971.

KEMPF, M.; AHIRWAL, D.; CZIEP, M. e WILHELM, M. Synthesis and Linear and Nonlinear Melt Rheology of Well-Defined Comb Architectures of PS and PpMS with a Low and Controlled Degree of Long-Chain Branching. Macromolecules, v. 46, 4978-994, 2013.

KIM, S. and KARRILA, S. Microhydrodynamics: principles and selected applications. Butterworth Heinemann, Boston, MA, 1992.

KIND, M.; PEUKERT, W. and REHAGE, H. Colloid Process Engineering. Springer, 2015.

KOELLER, R.C. Application of fractional calculus to the theory of viscoelasticity. Journal of Applied Mechanics, v. 51, 229-307, 1950.

KRIEGER, I. and DOUGHERTY, T. A mechanism for non-Newtonian flow in suspensions of rigid spheres. Transactions of the Society of Rheology, v. 3, n. 1, 137-152, 1959. 
LADOUX, B. and MEGE, R.M. Mechanobiology of collective cell behaviours. Nature Reviews Molecular Cell Biology, v. 18, 743-757, 2017.

LANDAU, L.D. and LIFSHITZ, E.M. Fluid Mechanics. Pergamon Press, 1998.

LARSON, R.G. Constitutive Equations for Polymer Melts and Solutions. Elsevier, 1988.

LAUGA, E. Propulsion in a viscoelastic fluid. Physics of Fluids, v. 19, 083104, 2007.

LAUGA, E. and POWER, T. The hydrodynamics of swimming microorganisms. Reports on Progress in Physics, v. 72, 096601, 2009.

LAUGA, E. and POWERS, T.R. The hydrodynamics of swimming microorganisms. Reports on Progress in Physics, v. 72, 096601, 2009.

LAUN, H.M. Prediction of elastic strains of polymer melts inshear and elongation. Journal of Rheology, v. 30, 459-501, 1986.

LEE, H.; CHOI, M.; LEE, D.; KIM, H.; HWANG, H.; KIM, H.; PARK, S.; PAIK, Y. and LEE, J. Nictation, a dispersal behavior of the nematode Caenorhabditis elegans, is regulated by IL2 neurons. Nature Neuroscience, v. 15, n. 2, 107-112, 2011.

LEYGUE, A.; BAILLY, C. and KEUNINGS, R. A tube-based constitutive equation for polydisperse entangled linear polymers. Journal of Non-Newtonian Fluid Mechanics, v. 136, 1-16, 2006.

LIU, I.S. On Euclidean objectivity and the principle of material frame-indifference. Continuum Mechanics and Thermodynamics, v. 16, 177-183, 2004.

LIU, M.; ZENTGRAF, T.; LIU, Y.; BARTAL, G. and ZHANG, X. Light-driven nanoscale plasmonic motors. Nature Nanotechnology, v. 5, 570-573, 2010. 
LIVERPOOL, T. and MARCHETTI, M. Instabilities of Isotropic Solutions of Active Polar Filaments. Physics Review Letters, v. 90, n. 13, 138102, 2003.

LIVERPOOL, T. and MARCHETTI, M. Cell Motility. Springer, 2007.

LOGET, G. and KUHN, A. Propulsion of Microobjects by Dynamic Bipolar Self-Regeneration. Journal of the American Chemical Society, v. 132, 15918-15919, 2010.

LOPEZ, H.M.; GACHELIN, J.; DOUARCHE, C.; AURADOU, H. and CLEMENT, E. Turning bacteria suspensions into super fluids. Physical Review Letters, v. 115, 028301, 2015.

LUSHI, E.; GOLDSTEIN, R.E. and SHELLEY, M. Collective Chemotactic Dynamics in the Presence of Self-Generated Fluid Flows. Physical Review E, v. 86, 040902(R), 2012.

MACSPORRAN, W.C. and SPIERS, R.P. The dynamic performance of the Weissenberg rheogoniometer III. Large amplitude oscillatory shearing - harmonic analysis. Rheologica Acta, v. 23, 90-96, 1984.

MALADEN, R.D.; DING, Y.; LI, C. e GOLDMAN, D.I. Undulatory swimming in sand: subsurface locomotion of the sandfish lizard. Science, v. 325, 314, 2009.

MALKIN, A.Y. and ISAYEV, A.I. Rheology: Concepts, Methods and Applications. ChemTec Publishing, 2011.

MALVAR, S.; GONTIJO, R.G.; CARMO, B.S. e CUNHA, F.R. On the kinematics-wave motion of living particles in suspension. Biomicrofluidics, v. 11, n. 4, 044112, 2017.

MARENDUZZO, D.; ORLANDINI, E. and YEOMANS, J.M. Hydrodynamics and rheology of active liquid crystals: a numerical investigation. Physical Review Letters, v. 98, n. 112(49), 118102, 2007. 
MARON, S. and PIERCE, P. Application of Ree-Eyring generalized flow theory to suspensions of spherical particles. Journal of colloid science, v. 11, n. 1, 80-95, 1956.

MELITO, H.S.; DAUBERT, C.R. and FOEGEDING, E.A. Validation of a large amplitude oscillatory shear protocol. Journal of Food Engineering, v. 113, n. 1, 124-135, 2012.

MIKI, K. and CLAPHAM, D.E. Rheotaxis guides mammalian sperm. Current Biology, v. 23, 443-452, 2013.

MOONEY, M. The viscosity of a concentrated suspension of spherical particles. Journal of Colloid Science, v. 6, n. 2, 162-170, 1951.

MORAN, J. and POSNER, J. Phoretic self-propulsion. Annual Review of Fluid Mechanics, v. 49, 511-540, 2017.

NAM, J.G.; AHNA, K.H. and LEE, S.J. First normal stress difference of entangled polymer solutions in large amplitude oscillatory shear flow. Journal of Rheology, v. 54, 1243, 2010.

NEIDHOFER, T.M.; WILHELM, M. and DEBBAUT, B. Fourier-transform rheology experiments and finite-element simulations on linear polystyrene solutions. Journal of Rheology, v. 47, n. 6, 1351-1371, 2003.

NOVIKOV, V.V. Fractals, Diffusion, and Relaxation in Disordered Complex Systems, Part 2, chapter 7. Wiley-Interscience, 2006.

OLDROYD, J.G. On the Formulation of Rheological Equations of State. . Proceedings of the Royal Society A: Mathematical, Physical and Engineering Sciences, v. 200, 523-541, 1950.

OLIVEIRA, T.F. and CUNHA, F.R. A Theoretical Description of a Dilute Emulsion of Very Viscous Drops Undergoing Unsteady Simple Shear. Journal of Fluids Engineering, v. 133, n. 10, $101208,2011$. 
PARIDA, L.; FHOSH, U.U. and PADMANABHAN, V. The effects of groove height and substrate stiffness on C. elegans locomotion. Journal of Biomechanics, v. 55, 34-40, 2017.

PARIDA, L. and PADMANABHAN, V. Durotaxis in Nematode Caenorhabditis elegans. Biophysical Journal, v. 111, 666-674, 2016.

PAXTON, W.; KISTLER, K.; OLMEDA, C.; SEN, A.; ANGELO, S. et al.. Catalytic nanomotors: autonomous movement of striped nanorods. Journal of the American Chemical Society, v. 126, n. 41, 13424-13431, 2004.

PHAN-THIEN, N. and TANNER, R.I. A New Constitutive Equation Derived from Network Theory. Journal of Non-Newtonian Fluid Mechanics, v. 2, 353-365, 1977.

PHAN-THIEN, N. and TANNER, R.I. A Nonlinear Network Viscoelastic Model. Journal of Rheology, v. 22, 259-283, 1978.

PIRRI, J.K. and ALKEMA, M.J. The neuroethology of C. elegans escape. Current Ppinion in Neurobiology, v. 22, n. 2, 187-193, 2012.

POTOMKIN, M.; TOURNUS, M.; BERLYAND, L.V. e ARANSON, I.S. Flagella bending affects macroscopic properties of bacterial suspensions. Journal of the Royal Society Interface, v. 14, 20161031, 2017.

POZRIKIDIS, C. Fluid Dynamics: Theory, Computation, and Numerical Simulation. Springer Science+Business Media, LLC, 2011.

PURCELL, E.M. Life at low Reynolds number. American Journal of Physics, v. 45, n. 3, 1977.

RALLISON, J.M. Note on the Faxén relations for a particle in Stokes flow. Journal of Fluid Mechanics, v. 88, n. 3, 529-533, 1978. 
ROGERS, A.S. and LETTINGA, P. A sequence of physical processes determined and quantified in large-amplitude oscillatory shear (LAOS): Application to theoretical nonlinear models. Journal of Rheology, v. 56, n. 1, 1-25, 2012.

ROUSSLET, N.; SPRENGER, J.; TAPPAN, S.J. e GLASER, J.R. Robust tracking and quantification of $\mathrm{C}$. elegans body shape and locomotion through coiling, entanglement, and omega bends. Worm, v. 3, n. 4, e982437, 2014.

SAINTILlAN, D. Extensional rheology of active suspensions. Physical Review E, v. 81, n. 5, 056307, 2010.

SAINTILlAN, D. Rheology of Active Fluids. Annual Review of Fluid Mechanics, v. 50, 563592, 2018.

SAINTILLAN, D. and SHELLEY, M. Instabilities and Pattern Formation in Active Particle Suspensions: Kinetic Theory and Continuum Simulations. Physical Review Letters, v. 100, n. 17, $178103,2008$.

SAINTILLAN, D. and SHELLEY, M. Emergence of coherent structures and large-scale flows in motile suspensions. Journal of the Royal Society Interface, v. 9, n. 68, 571-585, 2011.

SANSONETTI, P. Host-pathogen interactions: the seduction of molecular cross talk. Gut, v. 50, III2-8, 2002.

SARAMITO, P. On a modified non-singular log-conformation formulation for Johnson-Segalman viscoelastic fluids. Journal of Non-Newtonian Fluid Mechanics, pp. 16-30, 2014.

SARKAS, I.; MAVRIDIS, D.; PAPAMICHAIL, M. e PAPADOPOULOS, G. Large and small signal distortion analysis using modified Volterra series. Analog Integrated Circuits and Signal Processing, v. 54, 133-142, 2008.

SERRA, D.O.; RICHTER, A.M.; KLAUCK, G.; MIKA, F. and HENGGE, R. Microanatomy at 
cellular resolution and spatial order of physiological differentiation in a bacterial biofilm. mBio, v. 86, e00103-13, 2013.

SHAPERE, A. and WILCZEK, F. Self-Propulsion at Low Reynolds Number. Physical Review Letters, v. 58, 2051-2054, 1987.

SHUKLA, A. and M., J.Y. Boltzmann superposition principle for a time-dependent soft material: assessment under creep flow field. Rheologica Acta, v. 56, 927-940, 2017.

SIGINER, D.A. Developments in the Flow of Complex Fluids in Tubes. Springer, 2015.

SIHMA, R. and RAMASWAMY, S. Hydrodynamic Fluctuations and Instabilities in Ordered Suspensions of Self-Propelled Particles. Physical Review Letters, v. 89, n. 5, 058101, 2002.

SIMHA, R.A. and RAMASWAMY, S. Hydrodynamic Fluctuations and Instabilities in Ordered Suspensions of Self-Propelled Particles. Physical Review Letters, v. 89, n. 5, 058101, 2002.

SIMON, H. Neural Networks: A Comprehensive Foundation. Prentice Hall, 1998.

SOKOLOV, A. and ARANSON, I. Reduction of Viscosity in Suspension of Swimming Bacteria. PhysicaL Review Letters, v. 103, n. 14, 148101, 2009a.

SOKOLOV, A. and ARANSON, I. Reduction of viscosity in suspension of swimming bacteria. Physical Review Letters, v. 103, 148101, 2009 b.

SOKOLOV, A.; GOLDSTEIN, R.E.; FELDCHTEIN, F. and ARANSON, I.S. Enhanced mixing and spatial instability in concentrated bacterial suspensions. Physical Review E, v. 80, n. 3, 031903, 2009.

SOMMER, T.; DANZA, F.; BERG, J.; SENGUPTA, A.; CONSTANTINESCU, G.; TOKYAY, T.; BURGMANN, H.; DRESSLER, Y.; SEPULVEDA, S.; SCHUBERT, C.; TONOLLA, M. and 
WUEST, A. Bacteria-induced mixing in natural waters. Geophysical Research Letters, v. 44, n. 18, 9424-9432, 2017.

SPAGNOLIE, S.E. Complex Fluids in Biological Systems: Experiment, Theory, and Computation. Springer, 2015.

STADLER, F.J.; LEYGUE, A.; BURHIN, H. e BAILLY, C. The potential of large amplitude oscillatory shear to gain an insight into the long-chain branching structure of polymers. The 235th ACS National Meeting, pp. 121-122, 2008.

STIERNAGLE, T. Maintenance of C. elegans. WormBook: The Online Review of C. elegans Biology, 2006.

SUBRAMANIAN, G. and KOCH, D. Critical bacterial concentration for the onset of collective swimming. Jornal of Fluid Mechanics, , n. 632, 359-400, 2009.

SZNITMAN, J.; PUROHIT, P.; KRAJACIC, P.; LAMITINA, T. and ARRATIA, P. Material Properties of Caenorhabditis elegans Swimming at Low Reynolds Number. Biophysical Journal, v. 98, 617-626, 2010.

TAKATORI, S. and BRADY, J. Swim Pressure: Stress Generation in Active Matter. Soft Matter, v. 100, n. 47, 9433-9445, 2014.

TANAKA, T.; HOCHER, L.O. and BENEDEK, G.B. Spectrum of light scattered from a viscoelastic gel . Journal of Chemical Physics, v. 59, 5151, 1973.

TAYLOR, G.I. Analysis of the swimming of microscopic organisms. Proceedings of the Royal Society A, v. 209, n. 1099, 447, 1951.

TEE, T.T. and DEALY, J.M. Nonlinear viscoelasticity of polymer melts. Transactions of the Society of Rheology, v. 19, n. 4, 595-615, 1975. 
TONAR, J.; TU, Y. and RAMASWAMY, S. Hydrodynamics and phases of flocks. Annals of Physics, v. 318, n. 1, 170-244, 2005.

TOTTORI, S.; ZHANG, L.; QIU, F.; KRAWCZYK, K.K.; FRANCO-OBREGON, A. e NELSON, B.J. Magnetic helical micromachines: fabrication, controlled swimming, and cargo transport. Advanced Materials, v. 24, 811-816, 2012.

TRUESDELL, C. The Elements of Continuum Mechanics. Springer Verlag, 1966.

TRUESDELL, C. A First Course in Rational Continuum Mechanics. Academic Press, 1991. ISBN 978-0127013008.

TULSIANI, D. Introduction to Mammalian Reproduction. Springer, New York, 2012.

VAN DER WEL, C.; ALLAN, D.; KEIM, N. e CASWELL, T. Trackpy. 2010.

URL: http://soft-matter.github.io/trackpy/v0.4.1/

VICTORIA J. BUTLER, V.J.; BRANICKY, R.; YEMINI, E.; LIEWALD, J.F.; GOTTSCHALK, A.; KERR, R.A.; CHKLOVSKII, D.B. e SCHAFER, W.R. A consistent muscle activation strategy underlies crawling and swimming in Caenorhabditis elegans. Journal of the Royal Society Interface, v. 12, 20140963, 2015.

VIDAL-GADEA, A.; WARD, K.; BERON, C.; GHORASHIAN, N.; GOKCE, S.; RUSSELL, J.; TRUONG, N.; PARIKH, A.; GADEA, O. et al.. Magnetosensitive neurons mediate geomagnetic orientation in Caenorhabditis elegans. eLife, v. 4, e07493, 2015.

VOGEL, S. Life in moving fluids. Princeton University Press, 1994.

VOITURIEZ, R.; JOANNY, J. and PROST, J. Generic phase diagram of active polar films. Physical Review Letters, v. 96, n. 2, 028102, 2006. 
WAMBACQ, P. and SANSEN, W.M. Distortion analysis of analog integrated circuits, v. 45. Kluwer, 1998.

WANG, W.; CASTRO, L.A.; HOYOS, M. e E., M.T. Autonomous motion of metallic microrods propelled by ultrasound. ACS Nano, v. 6, 6122-6132, 2012.

WARE, R.W.; CLARK, D.; CROSSLAND, K. e RUSSELL, R. The nerve ring of the nematode Caenorhabditis elegans: Sensory input and motor output. Journal of Comparative Neurology, v. 162, n. 1, 71-110, 1975.

WENSINK, H.; DUNKEL, J.; HEIDENREICH, S.; DRESCHER, K.; GOLDSTEIN, R.E.; LOWEN, H. and YEOMANS, J.M. Meso-scale turbulence in living fluids. PNAS, v. 109, n. 36, 14308-14313, 2012.

WHITE, J.; SOUTHGATE, E.; THOMSON, J. e BRENNER, S. The structure of the nervous system of the nematode Caenorhabditis elegans. Philosophical Transactions of the Royal Society B, v. 314, n. 1165, 1-340, 1986.

WHITE, J.; SOUTHGATE, E.; THOMSON, J. e BRENNER, S. The immersed boundary method. Acta Numerica, v. 11, 479-517, 2002.

WIEDEMANN, A.; VIRLOGEUX-PAYANT, I.; CHAUSSE, A.M. and SCHIKORA, A. ND VELGE, P. Host-pathogen interactions: the seduction of molecular cross talk. Frontiers in Microbiology, v. 5, 791, 2015.

WILHELM, M.; MARING, D. and SPIESS, H.W. Fourier-transform rheology. Rheologica Acta, v. 37, 399-405, 1998.

WIOLAND, H.; LUSHI, E. and GOLDSTEIN, R.E. Directed collective motion of bacterial under channel confinement. New Journal of Physics, v. 18, 075002, 2015.

WU, K.; HISHAMUDA, J.; CHEN, D.; DECAMP, S.; CHANG, Y.; FERNANDEZ-NIEVES, A.; 
FRADEN, S. and DOGIC, Z. Transition from turbulent to coherent flows in confined threedimensional active fluids. Science, v. 355, eaal1979, 2017.

XIA, Q.; XIAO, H.; PAN, Y. e WANG, L. Microrheology, advances in methods and insights. Advances in Colloid and Interface Science, v. 257, 71-85, 2018.

YASUDA, K.; ARMSTRONG, R.C. and COHEN, R.E. Shear flow properties of concentrated solutions of linear and star branched polystyrenes. Rheologica Acta, v. 20, n. 2, 163-178, 1981.

YATES, C.; ERBAN, R.; ESCUDERO, C.; COUZIN, I.; BUHL, J.; KEVREKIDIS, I.; MAINI, P. and SUMPTER, D. Inherent noise can facilitate coherence in collective swarm motion. Proceedings of the National Academy of Sciences of the USA, v. 106, n. 14, 5464-5469, 2009.

ZHANG, L.; ABBOTT, J.J.; DONG, L.; KATOCHBIL, B.E.; BELL, D. and NELSON, B.J. Artificial bacterial flagella: Fabrication and magnetic control. Applied Physics Letters, v. 94, 064107, 2009.

ZHANG, Z.; LIU, J.; MERIANO, J.; RU, C.; XIE, S.; LUO, J. e SUN, Y. Human sperm rheotaxis: a passive physical process. Scientific Reports, v. 6, 23553, 2016. 


\section{APPENDICES}




\section{A Spectral Methods via Fast Fourier Transform (FFT)}

In this appendix, we discuss the pseudospectral method based on the Fast Fourier Transform (FFT). The pseudospectral method is an alternative to finite differences and finite elements for some classes of partial differential equations. The pseudospectral method is more limited than these other approaches in several ways. If the problem is not naturally periodic, it has to be reformulated to a periodic setting.

To solve the incompressivle Navier-Stokes equations via Fast Fourier Transform, we consider the vorticity formulation

$$
\begin{gathered}
\frac{\partial \boldsymbol{\omega}}{\partial t}+\nabla \times(\boldsymbol{\omega} \times \boldsymbol{u})=\nu \nabla \boldsymbol{\omega} \\
\nabla \cdot \boldsymbol{u}=0 .
\end{gathered}
$$

After some algebraic manipulation, equation A.1 is transformed into the following form

$$
\frac{\omega}{\partial t}+\boldsymbol{u} \cdot \nabla \boldsymbol{\omega}-\boldsymbol{\omega} \cdot \nabla \boldsymbol{u}=\nu \Delta \boldsymbol{\omega}
$$

Equation A.3 looks like a parabolic partial differential equation but with the extra term $\boldsymbol{\omega} \cdot \nabla \boldsymbol{u}$. Using the incompressibility condition and properties of vorticity in $2 \mathrm{D}$, this term is identically zero, giving the following form of the momentum equation in terms of vorticity

$$
\frac{\partial \boldsymbol{\omega}}{\partial t}+\boldsymbol{u} \cdot \nabla \boldsymbol{\omega}=\nu \Delta \boldsymbol{\omega}
$$

Next, we introduce the streamfunction, $\varphi$, as part of the vector potential for $\boldsymbol{u}$

$$
\boldsymbol{u}=\nabla \times \varphi \hat{k}
$$


Hence if we have the streamfunction, it is possible to obtain the components of the 2D fluid velocity, e.g.:

$$
u=\frac{\partial \varphi}{\partial y}
$$

and

$$
v=-\frac{\partial \varphi}{\partial x}
$$

Furthermore, taking $\nabla \times \nabla \times \varphi \hat{k}$, we obtain a Poisson problem for $\varphi$ in terms of $\boldsymbol{\omega}$,

$$
\Delta \varphi=-\omega
$$

If we are able to solve for the streamfunction, $\varphi$, from $\boldsymbol{\omega}$, we can then get $\boldsymbol{u}$ and it will automatically satisfy the incompressibility condition from the form of equation A.5. IB2s uses the following algorithm to solve Navier-Stokes equations:

○ Step 1: Solve the Poisson problem for the streamfunction, $\varphi^{n}$, from the previous time-step's vorticity $\omega^{n}$ i.e.,

$$
\hat{\varphi}_{i j}^{n}=\frac{\omega_{i j}^{n}}{\left.K_{X_{i}}^{2}+k_{Y_{j}}^{2}\right)},
$$

where $k_{X_{i}}$ and $k_{Y_{j}}$ are the Fourier wave-numbers.

○ Step 2: Next we compute the $\mathrm{x}, \mathrm{y}$-derivatives of the streamfunction, $\varphi^{n}$ and vorticity, $\boldsymbol{\omega}^{n}$ (in real space), then compute discretized, advection term, and finally transform the advection term into frequency space. To do this, we take the derivatives of streamfunction in frequency space and then the Inverse Fast Fourier Transverse. 


$$
\begin{gathered}
u_{i j}^{n}=\mathcal{F}^{-1} K_{Y} \hat{\varphi}_{i j}^{n} \\
v_{i j}^{n}=\mathcal{F}^{-1}-K_{X} \hat{\varphi_{i j}^{n}} \\
\omega_{x_{i j}}^{n}=\mathcal{F}^{-1} K_{X} \hat{\omega_{i j}^{n}} \\
\omega_{y_{i j}}^{n}=\mathcal{F}^{-1} K_{Y} \hat{\omega_{i j}^{n}}
\end{gathered}
$$

Once the quantities are in real space, it is possible to compute the advection term, $F_{a d v_{i j}}^{n}$, from equation A.4.

- Step 3: Finally we use the Crank-Nicholson scheme to update the streamfunction to the next time-step $\varphi^{n+1}$,

$$
\hat{\varphi}_{i j}^{n+1}=\frac{\left[1+\frac{\nu \Delta t}{2}\left(k_{X_{i j}}^{2}+k_{Y_{i j}}^{2}\right)\right] \hat{\varphi}_{i j}^{n}-\Delta t \hat{F}_{\mathrm{adv}_{i j}}^{n}}{1-\frac{\nu \Delta t}{2}\left(k_{X_{i j}}^{2}+k_{Y_{i j}}^{2}\right)}
$$

Note that this method is semi-implicit, explicitly discretizing the advective term, while implicitly discretizing the diffusive viscous term. The Crank-Nicholson scheme is second order accurate in time and is unconditionally stable for an array of parabolic problems. 


\section{B Discretizing Navier-Stokes equations}

IB2d uses finite difference approximations to discretize the Navier-Stokes equations on a fixed lattice, e.g., the Eulerian (fluid) grid. It follows the discretization defined as follows

$$
\begin{gathered}
\rho\left(\frac{\boldsymbol{u}^{k+1}-\boldsymbol{u}^{k}}{\Delta t}+S_{\Delta x}\left(\boldsymbol{u}^{k} \boldsymbol{u}^{k}\right)\right)-\boldsymbol{D}^{0} p^{k+1}=\mu \Sigma_{\alpha=1}^{2} D_{\alpha}^{+} D_{\alpha}^{-} \boldsymbol{u}^{k+1}+\boldsymbol{F}^{k} \\
\boldsymbol{D}^{0} \cdot \boldsymbol{u}^{k+1}=0
\end{gathered}
$$

where $\Delta t$ and $\Delta x$ are the tie-step and Eulerian meshwidth, respectively, and $\rho$ and $\mu$ are the density and kinematic viscosity of the fluid, respectively. $\boldsymbol{D}^{0}$ is the central differencing operator, defined as

$$
\boldsymbol{D}^{0}=\left(D_{1}^{0}, D_{2}^{0}\right)
$$

with

$$
\left(D_{\alpha}^{0}\right)(\boldsymbol{x})=\frac{\phi\left(\boldsymbol{x}+\Delta x \boldsymbol{e}_{\alpha}\right)-\phi\left(\boldsymbol{x}+\Delta x \boldsymbol{e}_{\alpha}\right)}{2 \Delta x},
$$

where $\left(e_{1}, e_{2}\right)$ is the standard basis in $\mathbb{R}^{2}$. The viscous term, given by $\sum_{\alpha=1}^{2} D_{\alpha}^{+} D_{\alpha}^{-} \boldsymbol{u}^{k+1}$ is a difference approximation to the Laplacian, where the $D_{\alpha}^{ \pm}$operators are the forward and backward approximations to $\partial / \partial x_{a}$. They are defined as

$$
\left(D_{\alpha}^{+}\right)(\boldsymbol{x})=\frac{\phi\left(\boldsymbol{x}+\Delta x \boldsymbol{e}_{\alpha}\right)-\phi(x)}{\delta x}
$$




$$
\left(D_{\alpha}^{-}\right)(\boldsymbol{x})=\frac{\phi(\boldsymbol{x})-\phi\left(\boldsymbol{x}-\Delta x \boldsymbol{e}_{\alpha}\right)}{\delta x}
$$

The skew-symmetic difference operator, $S_{\Delta x}$, serves as an approximation to the nonlinear advection term, $\boldsymbol{u} \cdot \nabla \boldsymbol{u}$, and is defined as follows

$$
S_{\Delta x}=\frac{1}{2}\left[\boldsymbol{u} \cdot \boldsymbol{D}_{\Delta x}^{0} \phi+\boldsymbol{D}_{\Delta x}^{0} \phi \cdot(\boldsymbol{u} \phi)\right]
$$

Using the discretizations proposed in equations B.4, B.5, B.6 and B.7, the equations B.1 and B. 2 are linear in $\boldsymbol{u}^{k+1}$ and $p^{k+1}$. To solve for $\boldsymbol{u}^{k+1}$ and $p^{k+1}$ from $\boldsymbol{u}^{k}, p^{k+1}$ and $F^{k}$, the Fast Fourier Transform (FFT) is used. As mentioned before, this assumes a periodic domain.

It is important to highlight that Navier-Stokes equations need not be discretized in this manner, however this discretization makes it easier to spread the Lagrangian forces to the Eulerian grid and move the Lagrangian structure at the local fluid velocity. 


\section{Spline Interpolant Coefficients for Swimmers}

In this supplemental section, we list the spline interpolation coefficients when varying $\left(p_{1}, p_{2}\right)$ for the swimmer.

\section{C.1 Symmetric $\left(p_{1}, p_{2}\right)$ coefficients}

1. $\left(p_{1}, p_{2}\right)=(0.1,0.9)$

$$
\begin{array}{lll}
a_{0}=0 & b_{0}=0.014 & c_{0}=-10.111 \\
a_{1}=0 & b_{1}=-0.417 & c_{1}=33.333 \\
a_{2}=0 & b_{2}=4.167 & c_{2}=-33.333 \\
a_{3}=11.111 & b_{3}=-2.778 & c_{3}=11.111
\end{array}
$$

2. $\left(p_{1}, p_{2}\right)=(0.2,0.8)$

$$
\begin{array}{lll}
a_{0}=0 & b_{0}=0.083 & c_{0}=-5.250 \\
a_{1}=0 & b_{1}=-1.250 & c_{1}=18.750 \\
a_{2}=0 & b_{2}=6.250 & c_{2}=-18.750 \\
a_{3}=6.250 & b_{3}=-4.167 & c_{3}=6.250
\end{array}
$$

3. $\left(p_{1}, p_{2}\right)=(0.3,0.7)$

$$
\begin{array}{lll}
a_{0}=0 & b_{0}=0.321 & c_{0}=-3.762 \\
a_{1}=0 & b_{1}=-3.214 & c_{1}=14.286 \\
a_{2}=0 & b_{2}=10.714 & c_{2}=-14.286 \\
a_{3}=4.762 & b_{3}=-7.143 & c_{3}=4.762
\end{array}
$$

4. $\left(p_{1}, p_{2}\right)=(0.4,0.6)$ 


$$
\begin{array}{lll}
a_{0}=0 & b_{0}=1.333 & c_{0}=-3.167 \\
a_{1}=0 & b_{1}=-10.000 & c_{1}=12.500 \\
a_{2}=0 & b_{2}=25.000 & c_{2}=-12.500 \\
a_{3}=4.167 & b_{3}=16.667 & c_{3}=4.167
\end{array}
$$

\section{C.2 Asymmetric $\left(p_{1}, p_{2}\right)$ coefficients}

1. $\left(p_{1}, p_{2}\right)=(0.1,0.9)$

$$
\begin{array}{lll}
a_{0}=0 & b_{0}=0.014 & c_{0}=-10.111 \\
a_{1}=0 & b_{1}=-0.417 & c_{1}=33.333 \\
a_{2}=0 & b_{2}=4.167 & c_{2}=-33.333 \\
a_{3}=11.111 & b_{3}=-2.778 & c_{3}=11.111
\end{array}
$$

2. $\left(p_{1}, p_{2}\right)=(0.1,0.7)$

$$
\begin{array}{lll}
a_{0}=0 & b_{0}=0.019 & c_{0}=-2.704 \\
a_{1}=0 & b_{1}=-0.556 & c_{1}=11.111 \\
a_{2}=0 & b_{2}=5.556 & c_{2}=-11.111 \\
a_{3}=14.286 & b_{3}=-4.233 & c_{3}=3.704
\end{array}
$$

3. $\left(p_{1}, p_{2}\right)=(0.1,0.5)$

$$
\begin{array}{lll}
a_{0}=0 & b_{0}=0.028 & c_{0}=-1.222 \\
a_{1}=0 & b_{1}=-0.833 & c_{1}=6.667 \\
a_{2}=0 & b_{2}=8.333 & c_{2}=-6.667 \\
a_{3}=20.0 & b_{3}=-7.778 & c_{3}=2.222
\end{array}
$$

4. $\left(p_{1}, p_{2}\right)=(0.1,0.3)$ 


$$
\begin{array}{lll}
a_{0}=0 & b_{0}=0.056 & c_{0}=-0.587 \\
a_{1}=0 & b_{1}=-1.667 & c_{1}=4.762 \\
a_{2}=0 & b_{2}=16.667 & c_{2}=-4.762 \\
a_{3}=33.333 & b_{3}=-22.222 & c_{3}=1.587
\end{array}
$$

